SERVIÇO DE PÓS-GRADUAÇÃO DO ICMC-USP

Data de Depósito:

Assinatura:

\title{
Sobre Singularidades Analíticas de Soluções de uma Classe de Campos Vetoriais no Toro
}

\author{
Leonardo Avila
}

Orientador: Prof. Dr. Sérgio Luís Zani

Dissertação apresentada ao Instituto de Ciências Matemáticas e de Computação - ICMC/USP, como parte dos requisitos para obtenção do título de Mestre em Ciências - Matemática.

USP - São Carlos

Agosto/2009 



\section{Sumário}

$\begin{array}{lll}1 & \text { Introdução } & 7\end{array}$

2 Distribuições Periódicas 11

2.1 Funções Testes Periódicas . . . . . . . . . . . . . . . . . . . . . 11

2.2 Distribuições Periódicas . . . . . . . . . . . . . . 16

2.3 T-Convolução . . . . . . . . . . . . . . . . . . 28

2.4 A Álgebra $\left(\mathcal{P}_{\mathrm{T}}^{\prime}\left(\mathbb{R}^{n}\right), *,+\right) \ldots \ldots \ldots \ldots \ldots \ldots$

3 Séries de Fourier em $\mathcal{P}_{\mathrm{T}}\left(\mathbb{R}^{n}\right)$ e $\mathcal{P}_{\mathrm{T}}^{\prime}\left(\mathbb{R}^{n}\right) \quad 39$

3.1 Série de Fourier em $\mathcal{P}_{\mathrm{T}}\left(\mathbb{R}^{n}\right) \ldots \ldots \ldots \ldots . \ldots \ldots$

3.2 Série de Fourier em $\mathcal{P}_{\mathrm{T}}^{\prime}\left(\mathbb{R}^{n}\right) \ldots \ldots \ldots \ldots . \ldots \ldots$

3.3 Série Parcial de Fourier em $\mathcal{P}_{\mathrm{T}}\left(\mathbb{R}^{n}\right) \ldots \ldots \ldots \ldots . \ldots \ldots$

3.4 Série Parcial de Fourier em $\mathcal{P}_{\mathrm{T}}^{\prime}\left(\mathbb{R}^{n}\right) \ldots \ldots \ldots \ldots$

4 Funções Analíticas Reais em $\mathbb{R}^{n} \quad 63$

4.1 Série de Potências . . . . . . . . . . . . . . . . 63

4.2 Funções Analíticas Reais . . . . . . . . . . . . . . . . . . 69

$\begin{array}{lll}5 & \text { Expansões Assintóticas } & 77\end{array}$

6 Hipoeliticidade Analítica Global $\quad 83$

6.1 Hipoeliticidade Analítica Global do Operador $L=\partial_{t}+c(t) \partial_{x}$ em $\mathcal{D}^{\prime}\left(\mathbb{T}^{2}\right) 83$

6.2 Resultados Preliminares . . . . . . . . . . . . . . . 84

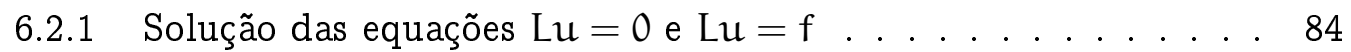

6.2.2 Automorfismos e Equivalências . . . . . . . . . . . . . . . . . 87

6.2.3 Majoração de Funções Analíticas Reais . . . . . . . . . . . . . . . 91

6.2.4 Caso de Coeficientes Constantes . . . . . . . . . . . . . . 92

6.3 Demonstração do Teorema Principal . . . . . . . . . . . . . . . . . . . 96

6.3 .1 Soluções Singulares . . . . . . . . . . . . . . . . . 99 


\section{Resumo}

O objetivo principal deste trabalho é o estudo da regularidade analítica global de certos operadores diferenciais definidos no toro. Uma ferramenta fundamental utilizada neste estudo são as séries parciais de Fourier, que nos permitem caracterizar tanto as distribuições periódicas quanto as funções analíticas reais periódicas através do comportamento assintótico de seus coeficientes parciais de Fourier. Neste sentido, apresentamos também um estudo detalhado das relações destes objetos com seus coeficientes parciais de Fourier. 


\begin{abstract}
The main goal of this work is to study global analytic regularity properties of certain differential operators acting in the torus. A main tool that will be used to achieve our goals are the partial Fourier series, which allow us to characterize objects such as periodic distributions or periodic real analytic functions in terms of the growth of their partial Fourier coefficients.
\end{abstract}




\section{Agradecimentos}

Ao professor Sérgio Zani, pela paciência impossível e ajuda ilegal, ao professor Adalberto Bergamasco, pelas decisivas intervenções (lema de Morse holomorfo por telefone!). Às pessoas que foram condição necessária (mas não suficiente): Fernado Gobbo, André Paulin, Welison Pantoso, Matheus Melo, Vinícius Antunes, Ton Marar (bendito telefone), Norival Zoppi, Camila Rodrigues, Natália Bellentane. Ao ICMC, pela excelente infraestrutura, me possilitando uma sólida formação. 


\section{Capítulo 1}

\section{Introdução}

O objetivo deste volume é o estudo da Hipoeliticidade Analítica Global de uma classe de operadores definidos no toro $\mathbb{T}^{2}$, a saber, $L=\partial_{t}+c(t) \partial_{x}$, onde $c(t)$ é uma função analítica real $2 \pi$-periódica. Tais operadores são considerados agindo no espaço $\mathcal{D}^{\prime}\left(\mathbb{T}^{2}\right)$ das distribuições $2 \pi$-periódicas definidas em $\mathbb{R}^{2}$, ou equivalentemente, distribuições definidas no toro $\mathbb{T}^{2}$. Dizer que um tal operador é globalmente analítico hipoelítico $(\mathrm{GAH})$ equivale a dizer que a imagem inversa do conjunto das funções analíticas reais em $\mathbb{T}^{2}$ está contida em tal conjunto. Assim, o objetivo dessa dissertação é responder à pergunta: Quais são os operadores $G A H$ da forma $L=\partial_{t}+c(t) \partial_{x}$ ? Ou seja, quais são as funções analíticas reais $c(t)$ em $\mathbb{T}$ de tal forma que L seja GAH? Este problema foi resolvido por Bergamasco em [B2].

Antes de nos aprofundarmos no estudo de [B2], será apresentado e analisado aqui um aparato teórico envolvido na formulação e resolução do problema.

No capítulo 1 é definido o espaço das distribuições T-periódicas, bem como o domínio (espaço das funções testes) de uma distribuição T-periódica, e são descritas propriedades resultantes da introdução de noções de convergência nesses espaços. São recuperadas para distribuições algumas operações permitidas a funções. É demonstrado um teorema que inclui linear e homeomorficamente o espaço das distribuições T-periódicas, $\mathcal{P}_{\mathrm{T}}^{\prime}\left(\mathbb{R}^{n}\right)$, no espaço das distribuições usuais $\mathcal{D}^{\prime}\left(\mathbb{R}^{n}\right)$. Por fim definimos e verificamos propriedades de T-convolução de distribuição por função uma função teste, e T-convolução de distribuições.

No capítulo 2 é construída uma teoria mínima, porém detalhadamente justificada, das séries (parciais) de Fourier. Distribuições T-periódicas e funções testes são caracterizadas através do crescimento de seus coeficientes (parciais) de Fourier.

No capítulo 3 são analisados elementos básicos para uma série de potências de várias variáveis bem como a definição de uma função analítica real de várias variáveis e algumas propriedades, em vista de estabelecermos um teorema que caracteriza, se periódicas, tais funções através de seus coeficientes (parciais) de Fourier.

No capítulo 4 é desenvolvido um método para obtenção de expansões assintóticas provenientes de integrais complexas dependendo de um parâmetro, o Método da 
Maior Descida (Steepest Descent). Tal método é usado para obtenção de uma expressão explícita para decaimento dos coeficientes parciais de Fourier de uma distribuição, o que permite obtermos soluções singulares para o operador $L=\partial_{t}+c(t) \partial_{x}$, no caso em que $c(t)$ não é do tipo que implica L GAH.

O capítulo 5 é inteiramente dedicado à demonstração do teorema principal da dissertação (teorema 6.3), que caracteriza os operadores GAH, da forma referida acima, definidos no toro $\mathbb{T}^{2}$. Na parte da demonstração em que é construída uma solução singular para o operador, ]apresentamos dois tipos de construção. Um deles é como em [B2] e o outro é baseado em [BNZ]. Procuramos preencher todos argumentos apresentados em [B2] e [BNZ], visando obter uma demonstração mais detalhada do teorema.

Antes de prosseguirmos, vamos estabelecer algumas definições e notações.

Definição 1.1 Seja $\mathbb{N}=\{0,1,2, \ldots\}$. Dizemos que $\alpha$ é um multi-índice, quando $\alpha \in \mathbb{N}^{n}$.

Definição 1.2 Sejam $\alpha=\left(\alpha_{1}, \ldots, \alpha_{n}\right) \in \mathbb{N}^{n}$ e $x=\left(x_{1}, \ldots, x_{n}\right) \in \mathbb{R}^{n}$. Defina

$$
\begin{gathered}
\alpha !=\alpha_{1} ! \alpha_{2} ! \cdots \alpha_{n} ! \\
|\alpha|=\alpha_{1}+\alpha_{2}+\cdots+\alpha_{m} \\
x^{\alpha}=x_{1}^{\alpha_{1}} x_{2}^{\alpha_{2}} \cdots x_{n}^{\alpha_{n}} \\
|x|^{\alpha}=\left|x_{1}\right|^{\alpha_{1}}\left|x_{2}\right|^{\alpha_{2}} \cdots\left|x_{n}\right|^{\alpha_{n}} \\
(x)_{\alpha}=\prod_{j=1}^{n}\left(x_{j}\right)_{\alpha_{j}}=\prod_{j=1}^{n}\left(x_{j}\left(x_{j}-1\right) \cdots\left(x-\alpha_{j}+1\right)\right) \\
\partial^{\alpha}=\frac{\partial^{\alpha}}{\partial x^{\alpha}}=\frac{\partial^{\alpha_{1}}}{\partial x_{1}^{\alpha_{1}}} \frac{\partial^{\alpha_{2}}}{\partial x_{2}^{\alpha_{2}}} \cdots \frac{\partial^{\alpha_{n}}}{\partial x_{n}^{\alpha_{n}}} \\
D^{\alpha}=\frac{1}{i|\alpha|} \frac{\partial^{\alpha}}{\partial x^{\alpha}} .
\end{gathered}
$$

Definição 1.3 Seja

$$
\mathrm{C}_{\mathrm{c}}^{\infty}\left(\mathbb{R}^{\mathrm{n}}\right)=\left\{\phi: \mathbb{R}^{\mathrm{n}} \longrightarrow \mathbb{C} ; \phi \in \mathrm{C}^{\infty}\left(\mathbb{R}^{\mathrm{n}}\right), \phi \text { tem suporte compacto }\right\},
$$

onde o suporte de $\phi, S(\phi)$, é o fecho do conjunto $\left\{x \in \mathbb{R}^{n} ; \phi(x) \neq 0\right\}$.

Definição 1.4 Seja $\left(\phi_{n}\right)_{n \in \mathbb{N}}$ uma sequência de funções em $\mathrm{C}_{\mathrm{c}}^{\infty}\left(\mathbb{R}^{\mathrm{n}}\right)$. Dizemos que $\phi_{\mathrm{n}} \longrightarrow 0$ em $\mathrm{C}_{\mathrm{c}}^{\infty}\left(\mathbb{R}^{\mathrm{n}}\right)$, quando existe um compacto $\mathrm{K}$ tal que $\mathrm{S}\left(\phi_{\mathrm{n}}\right) \subset \mathrm{K}$, para todo $\mathrm{n} \in \mathbb{N}$, e $\sup _{x \in \mathrm{A}}\left\{\left|\mathrm{D}^{\alpha} \phi_{\mathrm{n}}(\mathrm{x})\right|\right\} \longrightarrow 0, \mathrm{n} \longrightarrow \infty$, para todo $\alpha \in \mathbb{N}^{n}$. 
Definição 1.5 Seja u $: \mathbb{C}_{\mathrm{c}}^{\infty}\left(\mathbb{R}^{n}\right) \longrightarrow \mathbb{C}$ um funcional linear. Dizemos que u é continuo quando $\phi_{\mathrm{n}} \longrightarrow 0$ em $\mathrm{C}_{\mathrm{c}}^{\infty}\left(\mathbb{R}^{\mathrm{n}}\right)$ implica que $\mathrm{u}\left(\phi_{\mathrm{n}}\right) \longrightarrow 0 \mathrm{em} \mathbb{C}$.

Denotamos por

$$
\mathcal{D}^{\prime}\left(\mathbb{R}^{n}\right)=\left\{u: C_{c}^{\infty}\left(\mathbb{R}^{n}\right) \longrightarrow \mathbb{C} ; u \text { é linear e contínuo }\right\}
$$

o espaço das distribuições usuais em $\mathbb{R}^{n}$. 


\section{Capítulo 2}

\section{Distribuições Periódicas}

Distribuições são funcionais lineares contínuos definidos em espaços de funções a valores complexos. Uma distribuição periódica é um funcional linear contínuo, definido num espaço de funções periódicas suaves. Neste capítulo, vamos estabelecer o domínio de uma distribuição periódica, denotado por $\mathcal{P}_{\mathrm{T}}\left(\mathbb{R}^{n}\right)$, assim como a noção de convergência nesse espaço. Também analisaremos o fato de que, para cada função periódica em $\mathcal{P}_{\mathrm{T}}\left(\mathbb{R}^{n}\right)$, corresponde, de uma maneira simples, uma função de suporte compacto definida em $\mathbb{R}^{n}$. Após feito isso, estaremos aptos a falar em continuidade de funcionais definidos nesse espaço.

Em seguida, construímos o espaço $\mathcal{P}_{\mathrm{T}}^{\prime}\left(\mathbb{R}^{\mathfrak{n}}\right)$ das distribuições T-periódicas, bem como a noção de convergência para sequências de distribuições. Será demonstrado um teorema que permite enxergarmos $\mathcal{P}_{\mathrm{T}}^{\prime}\left(\mathbb{R}^{n}\right)$ como um subespaço de $\mathcal{D}^{\prime}\left(\mathbb{R}^{n}\right)$. Em seguida, estendemos de maneira apropriada, operações com funções para operações em distribuições periódicas. Verificamos que uma distribuição com derivada contínua corresponde a uma função derivável no sentido clássico.

Por fim definimos T-convolução de uma distribuição com uma função teste, e T-convolução entre distribuições, verificamos algumas propriedades e vemos como o estudo de operadores parciais diferenciais fica simplificada, a través da estrutura de álgebra comutativa em $\mathcal{P}_{\mathrm{T}}^{\prime}\left(\mathbb{R}^{n}\right)$, proveniente da T-convolução.

\subsection{Funções Testes Periódicas}

Definição 2.1 Sejam $\mathrm{T}>0, \mathrm{~m}=\left(\mathrm{m}_{1}, \ldots, \mathrm{m}_{\mathrm{n}}\right) \in \mathbb{Z}^{\mathrm{n}}$. Defina

$$
\mathcal{P}_{\mathrm{T}}\left(\mathbb{R}^{\mathrm{n}}\right)=\left\{\theta \in \mathrm{C}^{\infty}\left(\mathbb{R}^{\mathrm{n}}\right) ; \quad \theta(\mathrm{x})=\theta(\mathrm{x}-\mathrm{Tm}), \forall x \in \mathbb{R}^{\mathrm{n}}, \forall \mathrm{m} \in \mathbb{Z}^{\mathrm{n}}\right\} .
$$

$\mathcal{P}_{\mathrm{T}}\left(\mathbb{R}^{n}\right)$ é o espaço (vetorial) das funções testes periódicas.

Definição 2.2 Dizemos que uma sequência $\left(\theta_{j}\right)_{j \in \mathbb{N}}$, com $\theta_{j} \in \mathcal{P}_{T}\left(\mathbb{R}^{n}\right)$, converge em $\mathcal{P}_{\mathrm{T}}\left(\mathbb{R}^{n}\right)$, se existir $\theta \in \mathcal{P}_{\mathrm{T}}\left(\mathbb{R}^{n}\right)$, tal que $\mathrm{D}^{\alpha} \theta_{j}$ converge uniformemente para $\mathrm{D}^{\alpha} \theta$ em $\mathbb{R}^{n}$ para todo $\alpha=\left(\alpha_{1}, \ldots, \alpha_{n}\right) \in \mathbb{N}^{n}$. 
Com esta definição, $\mathcal{P}_{\mathrm{T}}\left(\mathbb{R}^{n}\right)$ é um espaço metrizável através da métrica

$$
\mathrm{d}(\theta, \phi)=\sum_{\mathrm{k}=0}^{\infty} \frac{p_{\mathrm{k}}(\theta-\phi)}{2^{\mathrm{k}}\left(1+p_{\mathrm{k}}(\theta-\phi)\right)},
$$

onde

$$
p_{k}(\theta)=\sup \left\{\left|D^{\alpha} \theta(x)\right| ; x \in \mathbb{R}^{n},|\alpha| \leq k\right\}
$$

é uma semi-norma.

Teorema 2.3 Uma sequência $\left(\theta_{j}\right)_{j \in \mathbb{N}}$, com $\theta_{j} \in \mathcal{P}_{T}\left(\mathbb{R}^{n}\right)$, converge para $\theta$ em $\mathcal{P}_{\mathrm{T}}\left(\mathbb{R}^{n}\right)$ se e somente se $\mathrm{p}_{\mathrm{k}}\left(\theta_{\mathrm{j}}-\theta\right) \longrightarrow 0, \mathrm{j} \longrightarrow \infty$, para todo $\mathrm{k} \in \mathbb{N}$.

Demonstração. Se $\theta_{j} \longrightarrow \theta$ em $\mathcal{P}_{\mathrm{T}}\left(\mathbb{R}^{n}\right)$ então, fixado $k \in \mathbb{N}$ e dado $\varepsilon>0$, existe $\mathrm{J}>0$ tal que se $j \geq \mathrm{J}$ e $x \in \mathbb{R}^{n}$, tem-se

$$
\left|\mathrm{D}^{\alpha} \theta_{\mathrm{j}}(x)-\mathrm{D}^{\alpha} \theta(x)\right|<\varepsilon
$$

para todo $\alpha$, tal que $|\alpha| \leq k$. Segue então que se $j \geq J$,

$$
p_{k}\left(\theta_{j}-\theta\right)=\sup _{\substack{x \in \mathbb{R}^{n} \\|\alpha| \leq k}}\left|D^{\alpha} \theta_{j}(x)-D^{\alpha} \theta(x)\right| \leq \varepsilon
$$

Se $p_{k}\left(\theta_{j}-\theta\right) \longrightarrow 0$ então para cada $\alpha \in \mathbb{N}^{n},|\alpha| \leq k$, segue que

$$
\sup _{x \in \mathbb{R}^{n}}\left|D^{\alpha} \theta_{j}(x)-D^{\alpha} \theta(x)\right| \leq \sup _{\substack{x \in \mathbb{R}^{n} \\|\beta| \leq k}}\left|D^{\beta} \theta_{j}(x)-D^{\beta} \theta(x)\right|=p_{k}\left(\theta_{j}-\theta\right) \longrightarrow 0 .
$$

Proposição 2.4 Para cada $\phi \in \mathrm{C}_{\mathrm{c}}^{\infty}\left(\mathbb{R}^{n}\right)$, defina

$$
\theta(x)=\sum_{m \in \mathbb{Z}^{n}} \phi(x-\operatorname{Tm})
$$

Então $\theta \in \mathcal{P}_{\mathrm{T}}\left(\mathbb{R}^{\mathfrak{n}}\right)$.

DemonstraçÃo. Apesar de não termos fixado ainda nenhum tipo de convergência para a série em questão, $\theta$ está bem definido, pois o fato de $\phi$ ter suporte compacto, implica que em cada ponto de seu domínio, $\theta$ é uma soma finita. De fato, existe $C>0$, tal que $\phi(x)=0$, para todo $x \in \mathbb{R}^{n}$ tal que $|x| \geq C$. Assim, se $x \in \mathbb{R}^{n}$ e $|x| \leq a$ para algum $a>0$, então

$$
\phi(x-\mathrm{Tm}) \neq 0 \Longrightarrow|x-\mathrm{Tm}|<\mathrm{C} \Longrightarrow\left|x_{i}-\mathrm{Tm}_{\mathrm{i}}\right|<\mathrm{C}, \quad i=1, \ldots, \mathrm{n} \Longrightarrow
$$




$$
\Longrightarrow-\mathrm{C}<\mathrm{Tm}_{\mathrm{i}}-x_{\mathrm{i}}<\mathrm{C}, \quad \mathrm{i}=1, \ldots, \mathrm{n} .
$$

Como $-a \leq x_{i} \leq a$, temos

$$
-\mathrm{C}-\mathrm{a} \leq-\mathrm{C}+\mathrm{x}_{\mathrm{i}}<\mathrm{Tm}_{\mathrm{i}}<\mathrm{C}+\mathrm{a}, \quad \mathrm{i}=1, \ldots, \mathrm{n} .
$$

Logo, $\left|m_{i}\right| \leq(C+a) / T, i=1, \ldots, n$ e, portanto, $|m| \leq n(C+a) / T$. Como existe apenas um número finito de $n$-uplas $m \in \mathbb{Z}^{n}$ que satisfazem essa última condição, podemos escrever, para algum $k=k(a) \in \mathbb{N}$ conveniente,

$$
\theta(x)=\phi\left(x-\mathrm{Tm}_{1}\right)+\cdots+\phi\left(x-\mathrm{Tm}_{\mathrm{k}}\right),
$$

desde que $|x| \leq a$.

Mostremos que $\theta$ é periódica. Tome $m \in \mathbb{Z}^{n}$,

$$
\begin{gathered}
\theta(x-T m)=\sum_{k \in \mathbb{Z}^{n}} \phi(x-T m-T k)=\sum_{k \in \mathbb{Z}^{n}} \phi(x-T(m+k))= \\
=\sum_{l \in \mathbb{Z}^{n}} \phi(x-\pi)=\theta(x) .
\end{gathered}
$$

Dado $x_{0} \in \mathbb{R}^{n}$, seja a $>0$ tal que $\left|x_{0}\right|<$ a. Assim,

$$
\theta(x)=\phi\left(x-\mathrm{Tm}_{1}\right)+\cdots+\phi\left(x-\mathrm{Tm}_{\mathrm{k}}\right), \quad|x|<\mathrm{a},
$$

e vemos que $\theta$ é infinitamente diferenciável em $x_{0}$, onde vale

$$
\mathrm{D}^{\alpha} \theta\left(x_{0}\right)=\mathrm{D}^{\alpha} \phi\left(\mathrm{x}_{0}-\mathrm{Tm}_{1}\right)+\cdots+\mathrm{D}^{\alpha} \phi\left(\mathrm{x}_{0}-\mathrm{Tm}_{\mathrm{k}}\right) .
$$

Deste modo, podemos escrever

$$
\mathrm{D}^{\alpha} \theta(x)=\sum_{m \in \mathbb{Z}^{n}} \mathrm{D}^{\alpha}(x-\mathrm{Tm})
$$

e $\theta \in \mathcal{P}_{\mathrm{T}}\left(\mathbb{R}^{\mathrm{n}}\right)$

Proposição 2.5 Seja $\left(\phi_{l}\right)_{l \in \mathbb{N}}, \phi_{l} \in C_{c}^{\infty}\left(\mathbb{R}^{n}\right)$, tal que $\phi_{l}$ converge para $\phi$ em $C_{c}^{\infty}\left(\mathbb{R}^{n}\right)$. Então a sequência $\left(\theta_{l}\right)_{l \in \mathbb{N}}$ em $\mathcal{P}_{\mathrm{T}}\left(\mathbb{R}^{n}\right)$, definida por $\theta_{\mathrm{l}}(\mathrm{x})=$ $\sum_{m \in \mathbb{Z}^{n}} \phi_{l}(x-T m)$, converge em $\mathcal{P}_{T}\left(\mathbb{R}^{n}\right)$ para $\theta(x)=\sum_{m \in \mathbb{Z}^{n}} \phi(x-T m)$.

Demonstração. Existe $a>0$ tal que $S\left(\phi_{l}\right) \subset B[0, a]$, para todo $l \in \mathbb{N}$ e $D^{\alpha} \phi_{l}$ converge para $\mathrm{D}^{\alpha} \phi$ uniformemente, para todo $\alpha \in \mathbb{N}^{n}$.

Sejam $\alpha \in \mathbb{N}^{n}$ e $\varepsilon>0$. Como $D^{\alpha}\left(\phi_{l}-\phi\right) \in C_{c}^{\infty}\left(\mathbb{R}^{n}\right)$, existem inteiros $m_{1}, \ldots, m_{k_{l}}$ tais que, para $x \in[0, T]^{n}$, temos 


$$
\sum_{m \in \mathbb{Z}^{\mathfrak{n}}} \mathrm{D}^{\alpha}\left(\phi_{l}-\phi\right)(x-\mathrm{Tm})=\mathrm{D}^{\alpha}\left(\phi_{l}-\phi\right)\left(x-\mathrm{Tm}_{1}\right)+\cdots+\mathrm{D}^{\alpha}\left(\phi_{l}-\phi\right)\left(x-\mathrm{Tm}_{\mathrm{k}_{l}}\right) .
$$

Como existe $a>0$ tal que $\phi_{l}(x)=0$, se $x \notin B[0, a]$, para algum $l \in \mathbb{N}$. Logo, se $\phi_{l}(x-\operatorname{Tm}) \neq 0$ para todo $l \in \mathbb{N}$, então $|x-T m| \leq a$ e isto implica que $x_{i}-a \leq$ $\mathrm{Tm}_{\mathrm{i}} \leq \mathrm{a}+\mathrm{x}_{\mathrm{i}}$.

Como $x \in[0, T]^{n}$, segue que $-a-T \leq-a \leq \operatorname{Tm}_{i}$ e $x_{i} \leq a+T$ e, portanto, $\left|m_{i}\right| \leq \frac{\mathrm{a}}{\mathrm{T}}+1$.

Como a independe de $l \in \mathbb{N}$, concluímos que só existe um número finito $k$, de índices $m \in \mathbb{N}$ tais que $\phi_{l}(x-\operatorname{Tm}) \neq 0$, para todo $l \in \mathbb{N}$. Deste modo, podemos escrever

$$
\begin{aligned}
& \sum_{m \in \mathbb{Z}^{n}} \mathrm{D}^{\alpha}\left(\phi_{l}-\phi\right)(x-T \mathrm{~m})=\mathrm{D}^{\alpha}\left(\phi_{l}-\phi\right)\left(x-\mathrm{Tm}_{1}\right)+ \\
& +\cdots+\mathrm{D}^{\alpha}\left(\phi_{l}-\phi\right)\left(x-\mathrm{Tm}_{\mathrm{k}}\right), \quad \forall \mathrm{l} \in \mathbb{N}, \quad \forall x \in[0, T]^{\mathrm{n}} .
\end{aligned}
$$

Como $\mathrm{D}^{\alpha} \phi_{\mathrm{l}}$ converge para $\mathrm{D}^{\alpha} \phi$ uniformemente, existe $l_{0} \in \mathbb{N}$ tal que $l \geq l_{0}$ implica que

$$
\sup _{x \in R^{n}}\left|D^{\alpha} \phi_{l}(x)-D^{\alpha} \phi(x)\right| \leq \frac{\varepsilon}{k} .
$$

Logo, se $l \geq l_{0}$ e $x \in[0, T]^{n}$ então

$$
\begin{gathered}
\left|\mathrm{D}^{\alpha} \theta_{l}(x)-\mathrm{D}^{\alpha} \theta(x)\right|=\left|\mathrm{D}^{\alpha}\left(\theta_{l}-\theta\right)(x)\right|= \\
=\left|\mathrm{D}^{\alpha}\left(\sum_{\mathrm{m} \in \mathbb{Z}^{\mathfrak{n}}}\left(\phi_{l}-\phi\right)(x-\mathrm{Tm})\right)\right|=\left|\sum_{\mathrm{m} \in \mathbb{Z}^{\mathfrak{n}}} \mathrm{D}^{\alpha}\left(\phi_{l}-\phi\right)(x-\mathrm{Tm})\right|= \\
=\left|\mathrm{D}^{\alpha}\left(\phi_{l}-\phi\right)\left(x-\mathrm{Tm}_{1}\right)+\cdots+\mathrm{D}^{\alpha}\left(\phi_{l}-\phi\right)\left(x-\mathrm{Tm}_{\mathrm{k}}\right)\right| \leq \\
\leq\left|\mathrm{D}^{\alpha}\left(\phi_{l}-\phi\right)\left(x-\mathrm{Tm}_{1}\right)\right|+\cdots+\left|\mathrm{D}^{\alpha}\left(\phi_{l}-\phi\right)\left(x-\mathrm{Tm}_{\mathrm{k}}\right)\right| \leq \\
\leq \frac{\varepsilon}{\mathrm{k}}+\cdots+\frac{\varepsilon}{\mathrm{k}}=\varepsilon .
\end{gathered}
$$

Definição 2.6 Seja

$$
\mathrm{U}_{\mathrm{T}}\left(\mathbb{R}^{\mathrm{n}}\right)=\left\{\xi \in \mathrm{C}_{\mathrm{c}}^{\infty}\left(\mathbb{R}^{\mathrm{n}}\right) ; \quad \sum_{\mathrm{m} \in \mathbb{Z}} \xi(x-\mathrm{Tm})=1, \quad \forall x \in \mathbb{R}^{\mathrm{n}}\right\} .
$$

Se $\xi \in \mathrm{U}_{\mathrm{T}}\left(\mathbb{R}^{n}\right)$, diremos que $\xi$ é unitária.

Exemplo 2.7 Tome $\mathrm{T}=1$. 
Defina $\xi: \mathbb{R} \longrightarrow \mathbb{R}$, por

$$
\xi(t)=\chi_{(-1,1)}\left(\int_{0}^{1} e^{\frac{-1}{x(1-x)}} d x\right)^{-1} \int_{|t|}^{1} e^{\frac{-1}{x(1-x)}} d x
$$

Note que $\xi \in C_{c}^{\infty}\left(\mathbb{R}^{n}\right)$ e $S(\xi)=[-1,1]$. Dado $t \in \mathbb{R}$, seja $m_{0} \in \mathbb{Z}$ tal que $\mathrm{m}_{0} \leq \mathrm{t}<\mathrm{m}_{0}+1$, isto é, $0 \leq \mathrm{t}-\mathrm{m}_{0}<1$. Também, $-1 \leq \mathrm{t}-\mathrm{m}_{0}-1<0$.

Se $m \geq m_{0}+2$ então $t \leq m e$

$$
|t-m|=t-m \geq t+1-m_{0}=\left(t-m_{0}\right)+1 \geq 1 .
$$

Além disso, se $m<m_{0}$ então

$$
t-m=\left(t-m_{0}\right)+\left(m_{0}-m\right) \geq 1 .
$$

Logo, $\xi(t-m)=0$ se $m \notin\left\{m_{0}, m_{0}+1\right\}$.

Assim,

$$
\begin{gathered}
\sum_{m=-\infty}^{\infty} \xi(t-m)=\xi\left(t-m_{0}\right)+\xi\left(t-m_{0}-1\right)= \\
=\left(\int_{0}^{1} e^{\frac{-1}{x(1-x)}} d x\right)^{-1}\left(\int_{t-m_{0}}^{1} e^{\frac{-1}{x(1-x)}} d x+\int_{1+m_{0}-t}^{1} e^{\frac{-1}{x(1-x)}} d x\right)= \\
=\left(\int_{0}^{1} e^{\frac{-1}{x(1-x)}} d x\right)^{-1}\left(\int_{t-m_{0}}^{1} e^{\frac{-1}{x(1-x)}} d x\right)-\int_{t-m_{0}}^{0} e^{\frac{-1}{x(1-x)}} d x= \\
=\left(\int_{0}^{1} e^{\left.\frac{-1}{x(1-x)} d x\right)^{-1}\left(\int_{0}^{1} e^{\frac{-1}{x(1-x)}} d x\right)=1 .}\right.
\end{gathered}
$$

Com isso é possível obter uma função unitária $\zeta: \mathbb{R}^{n} \longrightarrow \mathbb{C}$ dada por

$$
\zeta\left(x_{1}, \ldots, x_{n}\right)=\xi\left(x_{1}\right) \cdots \xi\left(x_{n}\right) .
$$

Proposição 2.8 Seja $\xi$ uma função unitária. Se $\left(\theta_{j}\right)_{j \in \mathbb{N}}$ converge para $\theta$ em $\mathcal{P}_{\mathrm{T}}\left(\mathbb{R}^{n}\right)$ então $\left(\xi \theta_{j}\right)_{j \in \mathbb{N}}$ converge para $\xi \theta$ em $\mathrm{C}_{\mathrm{c}}^{\infty}\left(\mathbb{R}^{n}\right)$.

DemonstraçÃo. Temos que $S\left(\xi \theta_{j}\right) \subset S(\xi)$. Dado $\alpha \in \mathbb{N}^{n}$, temos que

$$
\mathrm{D}^{\alpha}\left(\xi \theta_{j}\right)=\sum_{\gamma+\beta=\alpha} \frac{\alpha !}{\gamma ! \beta !} \mathrm{D}^{\gamma} \xi \mathrm{D}^{\beta} \theta_{j}
$$

converge uniformemente para

$$
\sum_{\gamma+\beta=\alpha} \frac{\alpha !}{\gamma ! \beta !} D^{\gamma} \xi D^{\beta} \theta=D^{\alpha}(\xi \theta),
$$

pois $\theta_{j}$ converge para $\theta$ em $\mathcal{P}_{T}\left(\mathbb{R}^{\mathfrak{n}}\right)$. 
A proposição seguinte mostra que é sempre possível escrever uma função $\theta$ de $\mathcal{P}_{\mathrm{T}}\left(\mathbb{R}^{\mathrm{n}}\right)$ na forma $\sum_{m \in \mathbb{Z}} \phi(x-\mathrm{Tm})$, com $\phi \in \mathrm{C}_{\mathrm{c}}^{\infty}\left(\mathbb{R}^{\mathrm{n}}\right)$.

Proposição 2.9 A aplicação

$$
\begin{aligned}
\mathrm{F}: \mathrm{C}_{\mathrm{c}}^{\infty}\left(\mathbb{R}^{\mathfrak{n}}\right) & \longrightarrow \mathcal{P}_{\mathrm{T}}\left(\mathbb{R}^{\mathfrak{n}}\right) \\
\phi & \longmapsto \sum_{\mathrm{m} \in \mathbb{Z}} \phi(\cdot-\mathrm{Tm})
\end{aligned}
$$

é sobrejetora.

Demonstração. Dada $\theta \in \mathcal{P}_{\mathrm{T}}\left(\mathbb{R}^{\mathfrak{n}}\right)$, tome qualquer $\xi \in \mathrm{U}_{\mathrm{T}}\left(\mathbb{R}^{\mathrm{n}}\right)$ e defina $\phi=\xi \theta \in$ $\mathrm{C}_{\mathrm{c}}^{\infty}\left(\mathbb{R}^{n}\right)$. Assim, temos

$$
\begin{gathered}
(F \phi)(x)=\sum_{m \in \mathbb{Z}} \phi(x-T m)=\sum_{m \in \mathbb{Z}} \xi(x-T m) \theta(x-T m)= \\
=\theta(x) \sum_{m \in \mathbb{Z}} \xi(x-T m)=\theta(x) .
\end{gathered}
$$

Logo, $\mathrm{F} \phi=\theta$.

\subsection{Distribuições Periódicas}

Definição 2.10 Um funcional linear $f: \mathcal{P}_{\mathrm{T}}\left(\mathbb{R}^{n}\right) \longrightarrow \mathbb{C}$ é dito continuo (ou sequencialmente contínuo) se para toda sequência $\left(\theta_{j}\right)_{j \in \mathbb{N}}$ em $\mathcal{P}_{\mathrm{T}}\left(\mathbb{R}^{\mathrm{n}}\right)$ que satisfaz $\theta_{j} \longrightarrow 0$, implica que $f\left(\theta_{j}\right) \longrightarrow 0$ em $\mathbb{C}$.

Definição 2.11 O espaço vetorial

$$
\mathcal{P}_{\mathrm{T}}^{\prime}\left(\mathbb{R}^{n}\right)=\left\{f: \mathcal{P}_{\mathrm{T}}\left(\mathbb{R}^{n}\right) \longrightarrow \mathbb{C} ; \text { fé linear e continuo }\right\}
$$

é chamado de espaço das distribuições periódicas e um elemento seu é dito uma distribuição periódica.

Se $f \in \mathcal{P}_{T}^{\prime}\left(\mathbb{R}^{n}\right)$ e $\theta \in \mathcal{P}_{T}\left(\mathbb{R}^{n}\right)$, usaremos a seguinte notação: $f(\theta)=(f, \theta)$.

Teorema 2.12 Seja $f: \mathcal{P}_{\mathrm{T}}\left(\mathbb{R}^{n}\right) \longrightarrow \mathbb{C}$ um funcional linear. São equivalentes

1. fé continuo.

2. Existem $\mathrm{C}>0$ e $\mathrm{m} \in \mathbb{N}$ tais que

$$
|(f, \theta)| \leq C \sum_{|\alpha| \leq m} \sup _{x \in \mathbb{R}^{n}}\left|D^{\alpha} \theta(x)\right|,
$$

para toda $\theta$ de $\mathcal{P}_{\mathrm{T}}\left(\mathbb{R}^{n}\right)$. 
Demonstração. Suponha que 2 seja válido. Se $\left(\theta_{j}\right)_{j \in \mathbb{N}} \longrightarrow 0$ em $\mathcal{P}_{\mathrm{T}}\left(\mathbb{R}^{n}\right)$ então $\sup _{x \in \mathbb{R}^{n}}\left|D^{\alpha} \theta_{j}(x)\right|$ tende a zero para todo $\alpha \in \mathbb{N}^{n}$. Logo, a soma

$$
\sum_{|\alpha| \leq m} \sup _{x \in \mathbb{R}^{n}}\left|D^{\alpha} \theta_{j}(x)\right|
$$

também tende a zero quando $\mathrm{j}$ tende ao infinito. Portanto, usando a desigualdade em 2 concluímos que $f$ é contínuo.

Suponha agora que fé contínuo. Suponha que 2 é falso. Então, para cada $m \in \mathbb{N}$, podemos encontrar $\tilde{\theta}_{\mathrm{m}} \in \mathcal{P}_{\mathrm{T}}\left(\mathbb{R}^{n}\right)$ tal que

$$
\left|\left(f, \tilde{\theta}_{m}\right)\right|>m \sum_{|\alpha| \leq m} \sup _{x \in \mathbb{R}^{n}}\left|D^{\alpha} \tilde{\theta}_{m}(x)\right|
$$

Definindo $\theta_{m}=\frac{\tilde{\theta}_{m}}{\left|\left(f, \tilde{\theta}_{m}\right)\right|}$, temos que

$$
\left|\left(f, \theta_{m}\right)\right|=1>m \sum_{|\alpha| \leq m} \sup _{x \in \mathbb{R}^{n}}\left|D^{\alpha} \theta_{m}(x)\right| .
$$

Com isso, concluímos que

$$
0<\sum_{|\alpha| \leq m} \sup _{x \in \mathbb{R}^{n}}\left|D^{\alpha} \theta_{m}(x)\right|<\frac{1}{m} .
$$

Assim, $\theta_{m}$ converge para zero em $\mathcal{P}_{\mathrm{T}}\left(\mathbb{R}^{\mathfrak{n}}\right)$, porém $\left(f, \theta_{\mathrm{m}}\right)$ não converge a zero em $\mathbb{C}$.

Definição 2.14 Diremos que uma sequência $\left(f_{n}\right)_{n \in \mathbb{N}}$ de distribuições é convergente em $\mathcal{P}_{\mathrm{T}}^{\prime}\left(\mathbb{R}^{n}\right)$ se existir $\mathrm{f} \in \mathcal{P}_{\mathrm{T}}^{\prime}\left(\mathbb{R}^{n}\right)$ tal que para toda $\theta \in \mathcal{P}_{\mathrm{T}}\left(\mathbb{R}^{n}\right)$ a sequência de números complexos $\left(f_{n}, \theta\right)$ converge para $(f, \theta)$.

Convém notar que, de acordo com nossa definição 2.11, uma distribuição periódica não é uma distribuição usual em $\mathbb{R}^{n}$, pertencente a $\mathcal{D}^{\prime}\left(\mathbb{R}^{n}\right)$, de tal forma que $\mathcal{P}_{\mathrm{T}}^{\prime}\left(\mathbb{R}^{n}\right)$ e $\mathcal{D}^{\prime}\left(\mathbb{R}^{n}\right)$ são espaços distintos. Denotemos por $\langle u, \phi\rangle$ uma distribuição usual aplicada numa função $\phi \in \mathrm{C}_{\mathrm{c}}^{\infty}\left(\mathbb{R}^{n}\right)$ para diferenciar da notação $(f, \theta)$ usada no caso de uma distribuição periódica.

O próximo teorema mostra que $\mathcal{P}_{T}^{\prime}\left(\mathbb{R}^{n}\right)$ pode ser "colocado", linear e continuamente em $\mathcal{D}^{\prime}\left(\mathbb{R}^{n}\right)$, no sentido de que existe um homeomorfismo linear entre $\mathcal{P}_{T}^{\prime}\left(\mathbb{R}^{n}\right)$ e um subespaço vetorial e topológico de $\mathcal{D}^{\prime}\left(\mathbb{R}^{n}\right)$, a saber,

$$
\mathcal{D}_{\mathrm{T}}^{\prime}\left(\mathbb{R}^{\mathfrak{n}}\right)=\left\{u \in \mathcal{D}^{\prime}\left(\mathbb{R}^{\mathfrak{n}}\right) ; u=u_{T m}, \quad \forall \mathrm{m} \in \mathbb{Z}^{\mathfrak{n}}\right\}
$$

onde $\left\langle u_{T m}, \phi\right\rangle=\left\langle u, \phi_{-T m}\right\rangle$ e $\phi_{T m}(x)=\phi(x-T m)$. 
Teorema 2.15 Existe um homeomorfismo linear

$$
A: \mathcal{P}_{\mathrm{T}}^{\prime}\left(\mathbb{R}^{\mathrm{n}}\right) \longrightarrow \mathcal{D}_{\mathrm{T}}^{\prime}\left(\mathbb{R}^{\mathrm{n}}\right) .
$$

Demonstração. Seja $f \in \mathcal{P}_{\mathrm{T}}^{\prime}\left(\mathbb{R}^{\mathfrak{n}}\right)$. Para cada $\phi \in \mathrm{C}_{\mathrm{c}}^{\infty}\left(\mathbb{R}^{\mathfrak{n}}\right)$ considere

$$
\theta=\sum_{m \in \mathbb{Z}^{n}} \phi_{T m} .
$$

Note que $\theta \in \mathcal{P}_{\mathrm{T}}\left(\mathbb{R}^{n}\right)$. Podemos definir $u: C_{c}^{\infty}\left(\mathbb{R}^{n}\right) \longrightarrow \mathbb{C}$ por

$$
u(\phi)=\left(f, \sum_{m \in \mathbb{Z}^{\mathfrak{n}}} \phi_{T m}\right)=(f, \theta) .
$$

Mostremos que $u \in \mathcal{D}^{\prime}{ }_{\mathrm{T}}\left(\mathbb{R}^{\mathrm{n}}\right)$.

Linearidade: Se $\phi, \psi \in \mathrm{C}_{\mathrm{c}}^{\infty}\left(\mathbb{R}^{n}\right)$ e $\lambda \in \mathbb{C}$ então

$$
\begin{gathered}
u(\phi+\psi)=\left(f, \sum_{m \in \mathbb{Z}^{n}}(\phi+\psi)_{T m}\right)=\left(f, \sum_{m \in \mathbb{Z}^{n}} \phi_{T m}\right)+\left(f, \sum_{m \in \mathbb{Z}^{n}} \psi_{T m}\right) \\
=u(\phi)+u(\psi) . \\
u(\lambda \phi)=\left(f, \sum_{m \in \mathbb{Z}^{n}}(\lambda \phi)_{T m}\right)=\left(f, \lambda \sum_{m \in \mathbb{Z}^{n}} \phi_{T m}\right)=\lambda\left(f, \sum_{m \in \mathbb{Z}^{n}} \phi_{T m}\right)=\lambda u(\phi) .
\end{gathered}
$$

Continuidade: Se $\phi_{j}$ converge para zero em $C_{c}^{\infty}\left(\mathbb{R}^{n}\right)$, a proposição 2.5, página 13 , garante que $\theta_{j}=\sum_{m \in \mathbb{Z}^{n}}\left(\phi_{j}\right)_{T m}$ converge para 0 em $\mathcal{P}_{\mathrm{T}}\left(\mathbb{R}^{\mathfrak{n}}\right)$. Deste modo, $\left\langle u, \phi_{j}\right\rangle=$ $\left(f, \theta_{j}\right)$ converge para 0 em $\mathbb{C}$. Logo, $u \in \mathcal{D}^{\prime}\left(\mathbb{R}^{\mathfrak{n}}\right)$.

Periodicidade: Se $\phi \in \mathrm{C}_{\mathrm{c}}^{\infty}\left(\mathbb{R}^{\mathrm{n}}\right)$ e $\mathrm{m} \in \mathbb{Z}^{\mathrm{n}}$ então

$$
\begin{aligned}
& \left\langle u_{T m}, \phi\right\rangle=\left\langle u, \phi_{-T m}\right\rangle=\left(f, \sum_{k \in \mathbb{Z}^{\mathfrak{m}}}\left(\phi_{-T m}\right)_{T k}\right)= \\
& =\left(\mathrm{f}, \sum_{k \in \mathbb{Z}^{\mathfrak{m}}} \phi_{T(k-m)}\right)=\left(\mathrm{f}, \sum_{\mathrm{k} \in \mathbb{Z}^{\mathrm{m}}} \phi_{T \mathrm{k}}\right)=\langle\mathrm{u}, \phi\rangle,
\end{aligned}
$$

isto é, $\mathfrak{u}_{\mathrm{Tm}}=u$. Portanto, $u \in \mathcal{D}_{\mathrm{T}}^{\prime}\left(\mathbb{R}^{\mathfrak{n}}\right)$.

Seja

$$
\begin{aligned}
A: \mathcal{P}_{\mathrm{T}}^{\prime}\left(\mathbb{R}^{\mathrm{n}}\right) & \longrightarrow \mathcal{D}^{\prime}{ }_{\mathrm{T}}\left(\mathbb{R}^{\mathrm{n}}\right) \\
\mathrm{f} & \longmapsto u
\end{aligned}
$$

onde $\langle u, \phi\rangle=\left(f, \sum_{m \in \mathbb{Z}^{n}} \phi_{T m}\right), \phi \in C_{c}^{\infty}\left(\mathbb{R}^{n}\right)$.

Mostremos que $A$ é linear e contínuo.

Linearidade: Se $f, g \in \mathcal{P}_{\mathrm{T}}^{\prime}\left(\mathbb{R}^{\mathfrak{n}}\right)$ e $\lambda \in \mathbb{C}$ então, para toda $\phi \in \mathrm{C}_{\mathrm{c}}^{\infty}\left(\mathbb{R}^{\mathfrak{n}}\right)$, temos

$$
\langle A(f+g), \phi\rangle=\left(f+g, \sum_{m \in \mathbb{Z}^{n}} \phi_{T m}\right)=\left(f, \sum_{m \in \mathbb{Z}^{n}} \phi_{T m}\right)+\left(g, \sum_{m \in \mathbb{Z}^{n}} \phi_{T m}\right)=
$$




$$
\begin{gathered}
=\langle A(f), \phi\rangle+\langle A(g), \phi\rangle=\langle A(f)+A(g), \phi\rangle \\
\langle A(\lambda f), \phi\rangle=\left\langle\lambda f, \sum_{m \in \mathbb{Z}^{n}} \phi_{T m}\right\rangle=\lambda\left\langle f, \sum_{m \in \mathbb{Z}^{n}} \phi_{T m}\right\rangle=\lambda\langle A(f), \phi\rangle=\langle\lambda A(f), \phi\rangle .
\end{gathered}
$$

Continuidade: Considere uma sequência $\left(f_{j}\right)_{j \in \mathbb{N}}, f_{j} \in \mathcal{P}_{T}^{\prime}\left(\mathbb{R}^{n}\right)$ tal que $f_{j}$ tende a zero em $\mathcal{P}_{\mathrm{T}}^{\prime}\left(\mathbb{R}^{n}\right)$. Dada $\phi \in \mathrm{C}_{\mathrm{c}}^{\infty}$, temos que

$$
\lim _{j \rightarrow \infty}\left\langle A\left(f_{j}\right), \phi\right\rangle=\lim _{j \rightarrow \infty}\left(f_{j}, \sum_{m \in \mathbb{Z}^{\mathfrak{n}}} \phi_{T m}\right)=0 .
$$

Logo, $A\left(f_{j}\right)$ tende a zero em $\mathcal{D}^{\prime}{ }_{T}\left(\mathbb{R}^{n}\right)$.

Vamos agora, a partir de $u \in \mathcal{D}^{\prime}{ }_{T}\left(\mathbb{R}^{n}\right)$, definir uma única distribuição $T$-periódica $f=H(u)$, de modo que esta aplicação seja a inversa de $A$.

Seja $\xi \in U_{T}\left(\mathbb{R}^{n}\right)$. Para cada $u \in \mathcal{D}^{\prime}{ }_{T}\left(\mathbb{R}^{n}\right)$ defina

$$
\begin{aligned}
f_{\xi}: \mathcal{P}_{\mathrm{T}}\left(\mathbb{R}^{\mathrm{n}}\right) & \longrightarrow \mathbb{C} \\
\theta & \longmapsto\langle u, \xi \theta\rangle
\end{aligned} .
$$

Mostremos que $f_{\xi} \in \mathcal{P}_{T}^{\prime}\left(\mathbb{R}^{n}\right)$.

Linearidade: Sejam $\theta, \beta \in \mathcal{P}_{\mathrm{T}}\left(\mathbb{R}^{n}\right)$ e $\lambda \in \mathbb{C}$.

1. $f_{\xi}(\theta+\beta)=\langle u, \xi(\theta+\beta)\rangle=\langle u, \xi \theta+\xi \beta\rangle=\langle u, \xi \theta\rangle+\langle u, \xi \beta\rangle=f_{\xi}(\theta)+f_{\xi}(\beta)$.

2. $f_{\xi}(\lambda \theta)=\langle u, \xi(\lambda \theta)\rangle=\langle u, \xi \lambda \theta\rangle=\langle\lambda u, \xi \theta\rangle=\lambda\langle u, \xi \theta\rangle=\lambda f_{\xi}(\theta)$.

Continuidade: Seja $\left(\theta_{j}\right)_{j \in \mathbb{N}}, \theta_{j} \in \mathcal{P}_{T}\left(\mathbb{R}^{n}\right)$, uma sequência tal que $\theta_{j}$ tende a zero em $\mathcal{P}_{\mathrm{T}}\left(\mathbb{R}^{n}\right)$. A proposição 2.8 , página 15 , nos diz que $\xi \theta_{j}$ tende a zero em $\mathrm{C}_{\mathrm{c}}^{\infty}\left(\mathbb{R}^{\mathrm{n}}\right)$. Como $u \in \mathcal{D}^{\prime}{ }_{T}\left(\mathbb{R}^{n}\right)$, temos que $f_{\xi}\left(\theta_{j}\right)=\left\langle u, \xi \theta_{j}\right\rangle \longrightarrow 0$. Logo, $f_{\xi} \in \mathcal{P}_{T}^{\prime}\left(\mathbb{R}^{n}\right)$. Mostremos agora que a definição de $f_{\xi}$ independe da escolha de $\xi$, isto é, se $\xi, \eta \in U_{T}\left(R^{n}\right)$, então $f_{\xi}=f_{\eta}$. Mostremos antes que

$$
u=\sum_{m \in \mathbb{Z}^{\mathfrak{n}}}(u \eta)_{T m} \cdot
$$

Seja $\phi \in C_{c}^{\infty}\left(R^{n}\right)$. Então

$$
\begin{gathered}
\left\langle u-\sum_{|\mathfrak{m}| \leq k}(u \eta)_{T m}, \phi\right\rangle=\left\langle u-\sum_{|m| \leq k} u_{T m} \eta_{T m}, \phi\right\rangle= \\
=\left\langle u-\sum_{|m| \leq k} u \eta_{T m}, \phi\right\rangle=\left\langle u\left(1-\sum_{|m| \leq k} \eta_{T m}\right), \phi\right\rangle= \\
\left\langle u,\left(1-\sum_{|m| \leq k} \eta_{T m}\right) \phi\right\rangle,
\end{gathered}
$$


converge para zero, quando $k$ tende para infinito, pois $1-\sum_{|m| \leq k} \eta_{T m}$ tende a zero em $\mathcal{P}_{\mathrm{T}}\left(\mathbb{R}^{n}\right)$ e, pela proposição $2.8,\left(1-\sum_{|\mathrm{m}| \leq k} \eta_{T \mathrm{~m}}\right) \phi$ tende a zero em $\mathrm{C}_{\mathrm{c}}^{\infty}\left(\mathbb{R}^{\mathfrak{n}}\right)$. Além do mais, como

$$
\left\langle\sum_{|\mathrm{m}| \leq k}(u \eta)_{T m}, \phi\right\rangle=\sum_{|\mathrm{m}| \leq k}\left\langle(u \eta)_{T \mathfrak{m}}, \phi\right\rangle
$$

temos que

$$
\left\langle\sum_{m \in \mathbb{Z}^{n}}(u \eta)_{T m}, \phi\right\rangle=\sum_{m \in \mathbb{Z}^{n}}\left\langle(u \eta)_{T m}, \phi\right\rangle .
$$

Provemos agora que $f_{\xi}=f_{\eta}$. Dada $\theta \in \mathcal{P}_{\mathrm{T}}\left(\mathbb{R}^{n}\right)$, tome $\phi=\xi \theta$, e daí segue que

$$
\begin{aligned}
& f_{\xi}(\theta)=\langle u, \xi \theta\rangle=\left\langle\sum_{m \in \mathbb{Z}^{\mathfrak{n}}}(u \eta)_{T m}, \xi \theta\right\rangle= \\
= & \sum_{m \in \mathbb{Z}^{\mathfrak{n}}}\left\langle(u \eta),(\xi \theta)_{-T m}\right\rangle=\sum_{m \in \mathbb{Z}^{\mathfrak{n}}}\left\langle u \eta, \theta \xi_{-T m}\right\rangle= \\
= & \sum_{m \in \mathbb{Z}^{\mathfrak{n}}}\left\langle u, \eta \theta \xi_{-T m}\right\rangle=\sum_{m \in \mathbb{Z}^{\mathfrak{n}}}\left\langle u \xi_{-T m}, \eta \theta\right\rangle= \\
= & \sum_{m \in \mathbb{Z}^{\mathfrak{n}}}\left\langle(u \xi)_{-T m}, \eta \theta\right\rangle=\langle u, \eta \theta\rangle=f_{\eta}(\theta) .
\end{aligned}
$$

Passaremos a escrever somente $f_{\xi}=f$. Definamos

$$
\begin{aligned}
\mathrm{H}: \mathcal{D}^{\prime}{ }_{\mathrm{T}}\left(\mathbb{R}^{\mathfrak{n}}\right) & \longrightarrow \mathcal{P}_{\mathrm{T}}^{\prime}\left(\mathbb{R}^{\mathfrak{n}}\right) \\
\mathrm{u} & \longmapsto \mathrm{H}(\mathrm{u})
\end{aligned}
$$

onde

$$
\begin{aligned}
\mathrm{H}(\mathrm{u}): \mathcal{P}_{\mathrm{T}}\left(\mathbb{R}^{\mathfrak{n}}\right) & \longrightarrow \mathbb{C} \\
\theta & \longmapsto\langle u, \xi \theta\rangle
\end{aligned},
$$

para $\xi \in \mathrm{U}_{\mathrm{T}}$.

Linearidade: Dados $u, v \in \mathcal{D}_{T}^{\prime}\left(\mathbb{R}^{n}\right)$ e $\lambda \in \mathbb{C}$, temos para todo $\theta \in \mathcal{P}_{\mathrm{T}}\left(\mathbb{R}^{n}\right)$, que

$$
\begin{gathered}
(\mathrm{H}(\mathrm{u}+v), \theta)=\langle u+v, \xi \theta\rangle=\langle u, \xi \theta\rangle+\langle v, \xi \theta\rangle= \\
=(\mathrm{H}(\mathrm{u}), \theta)+(\mathrm{H}(v), \theta)=(\mathrm{H}(\mathrm{u})+\mathrm{H}(v), \theta) . \\
(\mathrm{H}(\lambda \mathrm{u}), \theta)=\langle\lambda u, \xi \theta\rangle=\lambda\langle u, \xi \theta\rangle=(\lambda \mathrm{H}(u), \theta) .
\end{gathered}
$$

Continuidade: Se $u_{j} \longrightarrow 0$ em $\mathcal{D}^{\prime}{ }_{T}\left(\mathbb{R}^{n}\right)$, então para cada $\theta \in \mathcal{P}_{\mathrm{T}}\left(\mathbb{R}^{n}\right)$ e $\xi \in \mathrm{U}_{\mathrm{T}}\left(\mathbb{R}^{n}\right)$, temos que $\left(\mathrm{H}\left(\mathrm{u}_{\mathrm{j}}\right), \theta\right)=\left\langle u_{j}, \xi \theta\right\rangle \longrightarrow 0$. Portanto, $\mathrm{H}\left(\mathrm{u}_{\mathrm{j}}\right) \longrightarrow 0$ em $\mathcal{P}_{\mathrm{T}}^{\prime}\left(\mathbb{R}^{\mathfrak{n}}\right)$.

Mostremos que $H$ é a inversa de $A$. Sejam $u \in \mathcal{D}^{\prime}{ }_{T}\left(\mathbb{R}^{n}\right), \phi \in C_{c}^{\infty}\left(R^{n}\right)$ e $\xi \in$ $U_{T}\left(\mathbb{R}^{n}\right)$. Observe que se $a>0$ é tal que $S(\xi) \subset B[0, a]$, então, se $|x|>a$, 


$$
\begin{gathered}
\psi(x)=\xi(x) \sum_{m \in \mathbb{Z}^{\mathfrak{n}}} \phi_{T \mathrm{~m}}(x)=\sum_{\mathrm{m} \in \mathbb{Z}^{\mathfrak{n}}} \xi \phi_{T \mathrm{~m}}(x)= \\
=\sum_{m \in \mathbb{Z}^{\mathfrak{n}}}\left(\phi \xi_{-T \mathrm{Tm}}\right)_{T \mathrm{~m}}(x)=0 .
\end{gathered}
$$

Se $|x| \leq a$, então

$$
\psi(x)=\xi(x)\left(\phi\left(x-\mathrm{Tm}_{1}\right)+\cdots+\phi\left(x-\mathrm{Tm}_{\mathrm{k}}\right)\right),
$$

certos $m_{1}, \ldots, m_{k} \in \mathbb{Z}^{n}$. Deste modo, para todo $x \in \mathbb{R}^{n}$, a série

$$
\sum_{m \in \mathbb{Z}^{\mathfrak{n}}} \xi \phi_{T m}=\sum_{m \in \mathbb{Z}^{\mathfrak{n}}}\left(\phi \xi_{-T m}\right)_{T m}
$$

é finita. Tendo em vista isso, obtemos

$$
\begin{aligned}
& \langle\mathrm{A}(\mathrm{H}(\mathrm{u})), \phi\rangle=\left(\mathrm{H}(\mathrm{u}), \sum_{\mathrm{m} \in \mathbb{Z}^{\mathfrak{n}}} \phi_{T_{\mathrm{m}}}\right)=\left\langle\mathrm{u}, \xi \sum_{\mathrm{m} \in \mathbb{Z}^{\mathfrak{n}}} \phi_{T_{\mathrm{m}}}\right\rangle= \\
& =\left\langle u, \sum_{m \in \mathbb{Z}^{\mathfrak{n}}}\left(\phi \xi_{-T m}\right)_{T m}\right\rangle=\sum_{m \in \mathbb{Z}^{\mathfrak{n}}}\left\langle u,\left(\phi \xi_{-T m}\right)_{T m}\right\rangle= \\
& =\sum_{m \in \mathbb{Z}^{\mathfrak{n}}}\left\langle u, \phi \xi_{-T m}\right\rangle=\sum_{m \in \mathbb{Z}^{\mathfrak{n}}}\left\langle u \xi_{-T m}, \phi\right\rangle= \\
& =\sum_{m \in \mathbb{Z}^{\mathfrak{n}}}\left\langle(u \xi)_{-T m}, \phi\right\rangle=\left\langle\sum_{m \in \mathbb{Z}^{n}}(u \xi)_{-T m}, \phi\right\rangle=\langle u, \phi\rangle,
\end{aligned}
$$

onde as duas últimas igualdades são válidas em virtude da observação feita antes de demonstrarmos que $f_{\xi}=f_{\eta}$.

Agora, para $f \in \mathcal{P}_{\mathrm{T}}^{\prime}\left(\mathbb{R}^{n}\right), \theta \in \mathcal{P}_{\mathrm{T}}\left(\mathbb{R}^{n}\right)$ e $\xi \in \mathrm{U}_{\mathrm{T}}\left(\mathbb{R}^{n}\right)$, vale o seguinte

$$
\begin{gathered}
(H(A(f)), \theta)=\langle A(f), \xi \theta\rangle=\left(f, \sum_{m \in \mathbb{Z}}(\xi \theta)_{T m}\right)= \\
=\left(f, \sum_{m \in \mathbb{Z}} \xi_{T m} \theta\right)=\left(f, \theta \sum_{m \in \mathbb{Z}} \xi_{T m}\right)=(f, \theta) .
\end{gathered}
$$

Portanto, $\mathrm{H}=\mathrm{A}^{-1}$, o que demonstra o teorema.

Exemplo 2.16 Toda função $\varphi: \mathbb{R}^{n} \longrightarrow \mathbb{C}$ tal que $\varphi(x)=\varphi(x-T m), \forall m \in \mathbb{Z}^{n}$, $\forall x \in \mathbb{R}^{n}$, desde que integrável em $[0, T]^{n}$, dá origem a uma distribuição periódica $f_{\varphi}: \mathcal{P}_{\mathrm{T}}\left(\mathbb{R}^{\mathfrak{n}}\right) \longrightarrow \mathbb{C}$, da seguinte maneira. Para todo $\theta \in \mathcal{P}_{\mathrm{T}}\left(\mathbb{R}^{\mathfrak{n}}\right)$, defina

$$
\left(f_{\varphi}, \theta\right)=\int_{[0, T]^{\mathfrak{n}}} \varphi \theta .
$$


A linearidade de $\mathrm{f}_{\varphi}$ segue da linearidade da integral. A continuidade de $\mathrm{f}_{\varphi}$ segue de

$$
\left|\int_{[0, T]^{n}} \varphi \theta_{j}\right| \leq \sup _{x \in[0, T]^{n}}\left|\theta_{j}(x)\right| \int_{[0, T]^{n}}|\varphi| .
$$

Exemplo 2.17 Considere

$$
\begin{aligned}
\delta: \mathcal{P}_{\mathrm{T}}\left(\mathbb{R}^{n}\right) & \longrightarrow \mathbb{C} \\
\theta & \longmapsto \theta(0) .
\end{aligned}
$$

Verifiquemos que $\delta$ é uma distribuição periódica.

Linearidade: Sejam $\theta_{1}, \theta_{2} \in \mathcal{P}_{\mathrm{T}}\left(\mathbb{R}^{n}\right)$ e $\lambda \in \mathbb{C}$. Temos

$$
\begin{aligned}
& \delta\left(\theta_{1}+\theta_{2}\right)=\left(\theta_{1}+\theta_{2}\right)(0)=\theta_{1}(0)+\theta_{2}(0)=\delta\left(\theta_{1}\right)+\delta\left(\theta_{2}\right) \\
& \delta\left(\lambda \theta_{1}\right)=\lambda \theta_{1}(0)=\lambda \delta\left(\theta_{1}\right)
\end{aligned}
$$

Continuidade: Se $\theta_{j} \longrightarrow 0$ em $\mathcal{P}_{\mathrm{T}}\left(\mathbb{R}^{n}\right)$, então $\delta\left(\theta_{\mathrm{j}}\right)=\theta_{\mathrm{j}}(0) \longrightarrow 0$ em $\mathbb{C}$. Deste modo, $\delta \in \mathcal{P}_{\mathrm{T}}^{\prime}\left(\mathbb{R}^{n}\right)$. Vejamos qual o correspondente de $\delta, A(\delta)$ em $\mathcal{D}^{\prime}{ }_{\mathrm{T}}\left(\mathbb{R}^{n}\right)$, segundo o teorema 2.15. Sabemos que tal correspondente, digamos $\mathrm{A}(\delta)=\tilde{\delta}$, é definido por

$$
\begin{gathered}
\langle\tilde{\delta}, \phi\rangle=\left(\delta, \sum_{m \in \mathbb{Z}^{\mathfrak{n}}} \phi_{T \mathrm{~m}}\right)=\sum_{\mathrm{m} \in \mathbb{Z}^{\mathfrak{n}}}\left(\delta, \phi_{T \mathrm{~m}}\right)= \\
=\sum_{\mathrm{m} \in \mathbb{Z}} \phi(\mathrm{Tm}) .
\end{gathered}
$$

$\tilde{\delta}$ é chamada de distribuição delta de Dirac T-periódica, ou delta de Dirac concentrada em $\mathrm{Tm}, \mathrm{m} \in \mathbb{Z}^{\mathrm{n}}$, lembrando que essa soma é finita para cada $\phi \in \mathrm{C}_{\mathrm{c}}^{\infty}\left(\mathbb{R}^{n}\right)$.

\section{Operações com Distribuições Periódicas}

Vejamos agora algumas operações permitidas a elementos de $\mathcal{P}_{T}^{\prime}\left(\mathbb{R}^{n}\right)$.

Sejam $f, g \in \mathcal{P}_{T}^{\prime}\left(\mathbb{R}^{n}\right), \theta, \theta_{1}, \theta_{2} \in \mathcal{P}_{T}\left(\mathbb{R}^{n}\right), \lambda \in \mathbb{C}$, e $\left(\beta_{j}\right)_{j \in \mathbb{N}}$, uma sequência em $\mathcal{P}_{\mathrm{T}}\left(\mathbb{R}^{\mathrm{n}}\right)$ que converge para zero.

\section{Adição.}

Defina

$$
\begin{array}{ccc}
f+g: \mathcal{P}_{\mathrm{T}}\left(\mathbb{R}^{n}\right) & \longrightarrow & \mathbb{C} \\
\theta & \longmapsto(f, \theta)+(g, \theta)
\end{array} .
$$


Linearidade: $(f+g)\left(\theta_{1}+\theta_{2}\right)=\left(f, \theta_{1}+\theta_{2}\right)+\left(g, \theta_{1}+\theta_{2}\right)=\left(f, \theta_{1}\right)+\left(f, \theta_{2}\right)+\left(g, \theta_{1}\right)+$ $\left(g, \theta_{2}\right)=\left(f+g, \theta_{1}\right)+\left(f+g, \theta_{2}\right)$.

$(f+g, \lambda \theta)=(f, \lambda \theta)+(g, \lambda \theta)=\lambda(f, \theta)+\lambda(g, \theta=\lambda((f, \theta)+(g, \theta))=$ $\lambda(f+g)(\theta)$.

Continuidade: $(\mathrm{f}+\mathrm{g})\left(\beta_{\mathrm{j}}\right)=\left(\mathrm{f}, \beta_{\mathrm{j}}\right)+\left(\mathrm{g}, \beta_{\mathrm{j}}\right) \longrightarrow 0, \mathrm{j} \longrightarrow 0$.

Logo $f+g \in \mathcal{P}_{\mathrm{T}}^{\prime}\left(\mathbb{R}^{\mathrm{n}}\right)$.

Multiplicação por $\phi \in \mathcal{P}_{\mathrm{T}}\left(\mathbb{R}^{n}\right)$.

Defina

$$
\begin{aligned}
\phi f: \mathcal{P}_{\mathrm{T}}\left(\mathbb{R}^{n}\right) & \longrightarrow c \\
\theta & \longmapsto(f, \phi \theta)
\end{aligned} .
$$

Linearidade: $(\phi f)\left(\theta_{1}+\theta_{2}\right)=\left(f, \phi\left(\theta_{1}+\theta_{2}\right)\right)=\left(f, \phi \theta_{1}+\phi \theta_{2}\right)=\left(f, \phi \theta_{1}\right)+\left(f, \phi \theta_{2}\right)=$ $\phi f\left(\theta_{1}\right)+\phi f\left(\theta_{2}\right)$.

$(\phi f)(\lambda \theta)=(f, \lambda \phi \theta)=\lambda(f, \phi \theta)=\lambda(\phi f)(\theta)$.

Continuidade: $(\phi f)\left(\beta_{j}\right)=\left(f, \phi \beta_{j}\right) \longrightarrow 0, j \longrightarrow 0$, pois, para cada $\alpha \in \mathbb{N}^{n}$, vale que

$$
\left|D^{\alpha} \phi \beta_{j}(x)\right| \leq \sup _{|\gamma| \leq|\alpha|}\left|D^{\gamma} \phi(x)\right| \sum_{|\gamma| \leq|\alpha|} C_{\gamma}\left|D^{\gamma} \beta_{j}(x)\right| \longrightarrow 0
$$

uniformemente para cada $\alpha$. Logo, $\phi f \in \mathcal{P}_{T}^{\prime}\left(\mathbb{R}^{n}\right)$.

\section{Translação.}

Seja $h \in \mathbb{R}^{n}$. Defina

$$
\begin{aligned}
f_{h}: \mathcal{P}_{\mathrm{T}}\left(\mathbb{R}^{n}\right) & \longrightarrow \mathbb{C} \\
\theta & \longmapsto\left(f, \theta_{-h}\right),
\end{aligned}
$$

onde $\theta_{h}(x)=\theta(x-h)$.

Linearidade: $f_{h}\left(\theta_{1}+\theta_{2}\right)=\left(f,\left(\theta_{1}+\theta_{2}\right)_{-h}\right)=\left(f,\left(\theta_{1}\right)_{-h}+\left(\theta_{2}\right)_{-h}\right)=\left(f,\left(\theta_{1}\right)_{-h}\right)+$ $\left(f,\left(\theta_{2}\right)_{-h}\right)=f_{h}\left(\theta_{1}\right)+f_{h}\left(\theta_{2}\right)$.

$f_{h}(\lambda \theta)=\left(f,(\lambda \theta)_{-h}\right)=\left(f, \lambda \theta_{-h}\right)=\lambda\left(f, \theta_{-h}\right)=\lambda f_{h}(\theta)$.

Continuidade: $f_{h}\left(\beta_{j}\right)=\left(f,\left(\beta_{j}\right)_{-h}\right) \longrightarrow 0$ em $\mathbb{C}$, pois para cada $\alpha \in \mathbb{N}^{n}$,

$$
D^{\alpha}\left(\beta_{j}\right)_{-h}(x)=D^{\alpha} \beta_{j}(x+h) \longrightarrow 0
$$

uniformemente. Logo, $f_{h} \in \mathcal{P}_{T}^{\prime}\left(\mathbb{R}^{n}\right)$. 


\section{Reflexão.}

Defina

$$
\begin{aligned}
\check{f}: \mathcal{P}_{\mathrm{T}}\left(\mathbb{R}^{n}\right) & \longrightarrow \mathbb{C} \\
\theta & \longmapsto(f, \check{\theta}),
\end{aligned}
$$

onde $\check{\theta}(x)=\theta(-x)$

Linearidade: $\check{\mathbf{f}}\left(\theta_{1}+\theta_{2}\right)=\left(f,\left(\theta_{1}+\theta_{2}\right)^{\sim}\right)=\left(f, \check{\theta_{1}}+\check{\theta_{2}}\right)=\left(f, \check{\theta_{1}}\right)+\left(f, \check{\theta_{2}}\right)=\check{f}\left(\theta_{1}\right)+\check{f}\left(\theta_{2}\right)$. $\check{f}(\lambda \theta)=\left(f,(\lambda \theta)^{\check{ }}\right)=(f, \lambda \check{\theta})=\lambda(f, \check{\theta})=\lambda \check{f}(\theta)$.

Continuidade: $\check{\mathrm{f}}\left(\beta_{\mathrm{j}}\right)=\left(\mathrm{f}, \check{\beta}_{\mathrm{j}}\right) \longrightarrow 0$ em $\mathbb{C}$, pois para cada $\alpha \in \mathbb{N}^{n}$, resulta em

$$
\sup _{x \in \mathbb{R}^{n}}\left|D^{\alpha} \beta_{j}(x)\right|=\sup _{x \in \mathbb{R}^{n}}\left|D^{\alpha} \check{\beta}_{j}(x)\right|,
$$

e deste modo $\check{\beta_{j}} \longrightarrow 0$ em $\mathcal{P}_{T}\left(\mathbb{R}^{n}\right)$. Logo, $\check{f} \in \mathcal{P}_{T}^{\prime}\left(\mathbb{R}^{n}\right)$.

Derivação de uma distribuição $f \in \mathcal{P}_{T}^{\prime}\left(\mathbb{R}^{n}\right)$.

Dada uma função teste $\theta \in \mathcal{P}_{\mathrm{T}}\left(\mathbb{R}^{n}\right)$, se $\alpha=\left(\alpha_{1}, \cdots, \alpha_{n}\right) \in \mathbb{N}^{n}$, temos que

$$
\mathrm{D}^{\alpha} \theta=\frac{1}{i^{|\alpha|}} \frac{\partial^{|\alpha|} \theta}{\partial x_{1}^{\alpha_{1}} \cdots \partial x_{n}^{\alpha_{n}}}
$$

É possível estender o conceito de derivada de uma função teste, de tal forma que toda distribuição periódica possua uma derivada que também é uma distribuição periódica.

Seja $f \in \mathcal{P}_{\mathrm{T}}^{\prime}\left(\mathbb{R}^{n}\right)$. Para cada $\alpha \in \mathbb{N}^{n}$, defina

$$
\begin{array}{rlc}
\mathrm{D}^{\alpha} \mathrm{f}: \mathcal{P}_{\mathrm{T}}\left(\mathbb{R}^{n}\right) & \longrightarrow & \mathbb{C} \\
\theta & \longmapsto\left(f,(-1)^{|\alpha|} \mathrm{D}^{\alpha} \theta\right)
\end{array}
$$

Linearidade:

$$
\begin{gathered}
D^{\alpha} f\left(\theta_{1}+\theta_{2}\right)=\left(f,(-1)^{|\alpha|} D^{\alpha}\left(\theta_{1}+\theta_{2}\right)\right)=\left(f,(-1)^{|\alpha|} D^{\alpha} \theta_{1}+(-1)^{|\alpha|} D^{\alpha} \theta_{2}\right)= \\
=\left(f,(-1)^{|\alpha|} D^{\alpha} \theta_{1}\right)+\left(f,(-1)^{|\alpha|} D^{\alpha} \theta_{2}\right)=D^{\alpha} f\left(\theta_{1}\right)+D^{\alpha} f\left(\theta_{2}\right) .
\end{gathered}
$$

Continuidade: $\mathrm{D}^{|\alpha|} f\left(\theta_{j}\right)=\left(\mathrm{f},(-1)^{\alpha} \mathrm{D}^{\alpha} \theta_{\mathrm{j}}\right) \longrightarrow 0$ em $\mathbb{C}$, pois $\mathrm{D}^{\alpha} \theta_{\mathrm{j}} \longrightarrow 0$ em $\mathcal{P}_{\mathrm{T}}\left(\mathbb{R}^{n}\right)$. Logo $D^{\alpha} f \in \mathcal{P}_{T}^{\prime}\left(\mathbb{R}^{n}\right)$.

Essa noção de derivada estende o conceito de derivada usual no seguinte sentido: s e $\theta \in \mathcal{P}_{\mathrm{T}}\left(\mathbb{R}^{n}\right)$ então devemos ter, usando a notação do exemplo 2.16, 


$$
D^{\alpha} f_{\theta}=f_{D^{\alpha} \theta} \text {. }
$$

De fato, se $\phi \in \mathcal{P}_{\mathrm{T}}\left(\mathbb{R}^{n}\right)$, então, usando integração por partes,

$$
\left(f_{D^{\alpha} \theta}, \phi\right)=\int_{[0, T]^{n}}\left(D^{\alpha} \theta\right) \phi=\int_{[0, T]^{n}} \theta(-1)^{|\alpha|} D^{\alpha} \phi=\left(D^{\alpha} f_{\theta}, \phi\right) .
$$

Operador derivação em $\mathcal{P}_{T}^{\prime}\left(\mathbb{R}^{n}\right)$.

Defina

$$
\begin{aligned}
\mathrm{D}^{\alpha}: \mathcal{P}_{\mathrm{T}}^{\prime}\left(\mathbb{R}^{\mathrm{n}}\right) & \longrightarrow \mathcal{P}_{\mathrm{T}}^{\prime}\left(\mathbb{R}^{\mathrm{n}}\right) \\
\mathrm{f} & \longmapsto \mathrm{D}^{\alpha} \mathrm{f}
\end{aligned}
$$

O Operador derivação em $\mathcal{P}_{\mathrm{T}}^{\prime}\left(\mathbb{R}^{\mathrm{n}}\right)$ é linear e contínuo.

Linearidade: Se $f, g \in \mathcal{P}_{\mathrm{T}}^{\prime}\left(\mathbb{R}^{n}\right)$ e $\lambda \in \mathbb{C}$, então para toda $\theta \in \mathcal{P}_{\mathrm{T}}\left(\mathbb{R}^{n}\right)$, temos

$$
\begin{aligned}
\left(D^{\alpha}(f+g), \theta\right)= & \left(f+g,(-1)^{|\alpha|} D^{\alpha} \theta\right)=\left(f,(-1)^{|\alpha|} D^{\alpha} \theta\right)+\left(g,(-1)^{|\alpha|} D^{\alpha} \theta\right)= \\
& =\left(D^{\alpha} f, \theta\right)+\left(D^{\alpha} g, \theta\right)=\left(D^{\alpha} f+D^{\alpha} g, \theta\right),
\end{aligned}
$$

e também

$$
\left(D^{\alpha}(\lambda f), \theta\right)=\left(\lambda f,(-1)^{|\alpha|} D^{\alpha} \theta\right)=\lambda\left(f,(-1)^{|\alpha|} D^{\alpha} \theta\right)=\lambda\left(D^{\alpha} f, \theta\right)=\left(\lambda D^{\alpha} f, \theta\right) .
$$

Continuidade: $D^{\alpha} f\left(\theta_{j}\right)=\left(f,(-1)^{\alpha} D^{\alpha} \beta_{j}\right) \longrightarrow 0$ em $\mathbb{C}$, pois $D^{\alpha} \beta_{j} \longrightarrow 0$ em $\mathcal{P}_{T}\left(\mathbb{R}^{n}\right)$.

Das definições acima, vale ressaltar que, quando uma distribuição $f_{\varphi}$ corresponde a $\varphi$ no sentido do exemplo 2.16, apesar dessa distribuição não ser formalmente $\varphi$, podemos tratá-la como tal, operacionalmente. Tal afirmação é justificada pelo fato de que, das operações acima, se $\sim: \mathcal{P}_{\mathrm{T}}^{\prime}\left(\mathbb{R}^{n}\right) \longrightarrow \mathcal{P}_{\mathrm{T}}^{\prime}\left(\mathbb{R}^{n}\right)$ é uma das operações unitárias acima e $*: \mathcal{P}_{\mathrm{T}}^{\prime}\left(\mathbb{R}^{n}\right) \times \mathcal{P}_{\top}^{\prime}\left(\mathbb{R}^{n}\right) \longrightarrow \mathcal{P}_{\mathrm{T}}^{\prime}\left(\mathbb{R}^{n}\right)$ é uma das operações binárias, valem

$$
\begin{aligned}
\widetilde{f_{\varphi}} & =f_{\tilde{\varphi}} \\
f_{\varphi_{1}} * f_{\varphi_{2}} & =f_{\varphi_{1} * \varphi_{2}} .
\end{aligned}
$$

Quando $\sim$ é alguma derivada, para podemos trocar $\widetilde{f_{\varphi}}$ por $\tilde{\varphi}$, é necessário que $\varphi$ seja derivável como função.

O próximo teorema garante que $\mathcal{P}_{\mathrm{T}}\left(\mathbb{R}^{n}\right)$ é denso no espaço das funções contínuas e T-periódicas. 
Teorema 2.18 Considere $\phi \in C_{c}^{\infty}\left(\mathbb{R}^{n}\right)$ tal que $\int \phi=1, \phi \geq 0$, $\mathrm{S}(\phi) \subset[0, \mathrm{~T}]^{\mathrm{n}}$. Seja

$$
\theta_{\varepsilon}=\varepsilon^{-n} \sum_{m \in \mathbb{Z}^{n}} \phi\left(\frac{x-\mathrm{Tm}}{\varepsilon}\right)
$$

$\operatorname{com} \theta_{\varepsilon} \in \mathcal{P}_{\mathrm{T}}\left(\mathbb{R}^{n}\right)$ e $0<\varepsilon<1$. Se f: $\mathbb{R}^{n} \longrightarrow \mathbb{C}$ é continua e T-periódica então

$$
f_{\varepsilon}(x)=\int_{[0, T]^{n}} f(x-y) \theta_{\varepsilon}(y) d y
$$

pertence $a \mathcal{P}_{\mathrm{T}}(\mathbb{R})^{\mathrm{n}}$ e $\mathrm{f}_{\varepsilon} \longrightarrow \mathrm{f}$ uniformemente.

Demonstração. Se $x \in[0, T]^{n}$, então para todo $m=\left(m_{1}, \ldots, m_{n}\right) \in \mathbb{Z}^{n}, m \neq 0$, existe $m_{i} \neq 0$ tal que

$$
-\frac{T m_{i}}{\varepsilon} \leq \frac{x_{i}-T m_{i}}{\varepsilon} \leq \frac{T\left(1-m_{i}\right)}{\varepsilon} .
$$

Se $m_{i} \leq 1$ então $\frac{x_{i}-T m_{i}}{\varepsilon} \leq 0$ e, deste modo, $\phi\left(\frac{x-T m}{\varepsilon}\right)=0$. Se $m_{i} \leq-1$, então $-\frac{T m_{i}}{\varepsilon} \geq \frac{T}{\varepsilon} \geq T$ e, portanto, $\phi\left(\frac{x-T m}{\varepsilon}\right)=0$. Assim, $\theta_{\varepsilon}(x)=\varepsilon^{-n} \phi\left(\frac{x}{\varepsilon}\right)$, se $x \in[0, T]^{n}$. Agora,

$$
\begin{aligned}
f_{\varepsilon}(x) & =\int_{[0, T]^{n}} f(x-y) \theta_{\varepsilon}(y) d y=\varepsilon^{-n} \int_{[0, T]^{n}} f(x-y) \phi\left(\frac{y}{\varepsilon}\right)(y) d y= \\
= & \int_{\left[0, T \varepsilon^{-1}\right]^{n}} f(x-\varepsilon z) \phi(z) d z=\int_{[0, T]^{n}} f(x-\varepsilon z) \phi(z) d z= \\
& =\int_{\mathbb{R}^{n}} f(x-\varepsilon z) \phi(z) d z=\varepsilon^{-n} \int_{\mathbb{R}^{n}} f(u) \phi\left(\frac{x-u}{\varepsilon}\right) d u,
\end{aligned}
$$

pois $S(\phi) \subset[0, T]^{n} \subset\left[0, T \varepsilon^{-1}\right]^{n}$. A igualdade

$$
f_{\varepsilon}(x)=\varepsilon^{-n} \int_{\mathbb{R}^{n}} f(y) \phi\left(\frac{x-y}{\varepsilon}\right) d y,
$$

nos permite escrever

$$
f_{\varepsilon}(x)=\varepsilon^{-n} \int_{K_{x}} f(y) \phi\left(\frac{x-y}{\varepsilon}\right) d y,
$$

onde

$$
\begin{gathered}
\mathrm{K}_{x}=\left\{\mathrm{y} \in \mathbb{R}^{\mathrm{n}} ; \quad 0 \leq \frac{x_{i}-y_{i}}{\varepsilon} \leq \mathrm{T}, \quad i=1, \ldots, n\right\}= \\
=\left\{y \in \mathbb{R}^{n} ; \quad x_{i}-\varepsilon \mathrm{T} \leq y_{i} \leq x_{i}, \quad i=1, \ldots, n\right\} .
\end{gathered}
$$

Note que $K_{x}$ é um conjunto compacto. Dados $x, x^{\prime} \in \mathbb{R}^{n}$, seja $K$ um compacto tal que $\mathrm{K}_{\mathrm{x}} \cup \mathrm{K}_{x^{\prime}} \subset \mathrm{K}$. Como $\phi$ é lipschitziana, existe $\mathrm{C}>0$ tal que 


$$
\begin{gathered}
\left|f_{\varepsilon}(x)-f_{\mathcal{\varepsilon}}\left(x^{\prime}\right)\right| \leq \int_{\mathbb{R}^{m}}|f(y)|\left|\phi\left(\frac{x-y}{\varepsilon}\right)-\phi\left(\frac{x^{\prime}-y}{\varepsilon}\right)\right| d y \leq \\
\leq \varepsilon^{-n} \sup _{u \in[0, T]^{n}}|f(u)| \int_{K}\left|\phi\left(\frac{x-y}{\varepsilon}\right)-\phi\left(\frac{x^{\prime}-y}{\varepsilon}\right)\right| d y \leq \\
\leq \varepsilon^{-n-1} C \sup _{u \in[0, T]^{n}}|f(u)| m(K)\left|x-x^{\prime}\right|,
\end{gathered}
$$

onde $m(K)$ é a medida de $K$. Logo $f_{\varepsilon}$ é contínua.

Para provar que $f_{\varepsilon}$ é diferenciável qualquer número de vezes, basta derivar sob o sinal de integração a função $\phi$ na expressão de $f_{\varepsilon}$ usada acima e repetir o procedimento acima usando $\mathrm{D}^{\alpha} \phi$ ao invés de $\phi$. Agora,

$$
\begin{aligned}
& \left|f(x)-f_{\varepsilon}(x)\right|=\left|\int_{[0, T]^{n}} f(x) \phi(z) d z-\int_{[0, T]^{n}} f(x-\varepsilon z) \phi(z) d z\right| \leq \\
& \quad \leq \int_{[0, T]^{n}}|f(x)-f(x-\varepsilon z)| \phi(z) d z \leq \sup _{z \in[0, T]^{n}}|f(x)-f(x-\varepsilon z)| .
\end{aligned}
$$

Como f é contínua e periódica, é uniformemente contínua. Assim, dado $\eta>0$, existe $\delta>0$ tal que

$$
|x-y|<\delta \Longrightarrow|f(x)-f(y)|<\eta
$$

Como $z \in[0, T]^{n},|z| \leq \sqrt{n} T$, e para todo $0<\varepsilon<\frac{\delta}{\sqrt{n} T}$ e $x \in \mathbb{R}^{n}$, temos que $|x-(x-\varepsilon z)|=\varepsilon|z|<\delta$. Logo $|f(x)-f(x-\varepsilon z)|<\eta$ e portanto

$$
\sup _{z \in[0, T]^{n}}|f(x)-f(x-\varepsilon z)| \leq \eta,
$$

o que prova $f_{\varepsilon} \longrightarrow f$ uniformemente.

No que segue, vamos justificar que, se a derivada de uma distribuição periódica é uma função contínua, então essa distribuição corresponde a uma função derivável.

Teorema 2.19 Se $\mathrm{f}$ e $\mathrm{g}$ são funções contínuas e periódicas em $\mathbb{R}^{n}$ e $\frac{\partial \mathrm{f}}{\partial x_{\mathrm{j}}}=\mathrm{g}$ no sentido de distribuições periódicas então f é diferenciável com relaçấo $a x_{j}$ como funções.

DEMONSTRAÇÃo. Usando a notação do teorema 2.18

$$
\begin{gathered}
\frac{\partial}{\partial x_{j}} f_{\varepsilon}(x)=\varepsilon^{-n} \int_{[0, T]^{n}} f(y) \frac{\partial}{\partial x_{j}}\left(\phi\left(\frac{x-y}{\varepsilon}\right)\right) d y= \\
=-\varepsilon^{-n} \int_{[0, T]^{n}} f(y) \frac{\partial}{\partial y_{j}}\left(\phi\left(\frac{x-y}{\varepsilon}\right)\right) d y=
\end{gathered}
$$




$$
=\varepsilon^{-n} \int_{[0, T]^{n}} g(y) \phi\left(\frac{x-y}{\varepsilon}\right)=g_{\varepsilon} .
$$

Como $f_{\varepsilon} \longrightarrow f$ e $g_{\varepsilon} \longrightarrow g$ uniformemente, segue que $\frac{\partial f}{\partial x_{j}}=g$.

\subsection{T-Convolução}

Definição 2.20 Sejam $f \in \mathcal{P}_{\mathrm{T}}^{\prime}\left(\mathbb{R}^{n}\right)$ e $\theta \in \mathcal{P}_{\mathrm{T}}\left(\mathbb{R}^{n}\right)$. Defina a convolução de $\mathrm{f}$ por $\theta$ como a função

$$
f * \theta: \mathbb{R}^{n} \longrightarrow \mathbb{C}
$$

dada por

$$
(f * \theta)(a)=\left(f, \check{\theta}_{a}\right)
$$

onde $\check{\theta}_{a}(x)=\theta(a-x)$.

Exemplo 2.21

Seja $\theta \in \mathcal{P}_{\mathrm{T}}\left(\mathbb{R}^{n}\right)$.

$$
(\delta * \theta)(a)=\left(\delta, \check{\theta}_{a}\right)=\check{\theta}_{a}(0)=\theta(a)
$$

\section{Exemplo 2.22}

Sejam $\theta \in \mathcal{P}_{\mathrm{T}}\left(\mathbb{R}^{n}\right)$ e $h \in \mathbb{R}^{n}$. Temos

$$
\left(\delta_{h} * \theta\right)(a)=\left(\delta_{h}, \check{\theta}\right)=\left(\delta,(\check{\theta})_{-h}\right)=\left(\check{\theta}_{a}\right)_{-h}(0)=\check{\theta}_{a}(h)=\theta(a-h) .
$$

Exemplo 2.23

Sejam $\alpha \in \mathbb{N}^{n}$ e $\theta \in \mathcal{P}_{\mathrm{T}}\left(\mathbb{R}^{n}\right)$. Temos

$$
\begin{gathered}
\left(\mathrm{D}^{\alpha} \delta * \theta\right)(\mathrm{a})=\left(\mathrm{D}^{\alpha} \delta, \check{\theta}_{\mathrm{a}}\right)=\left(\delta,(-1)^{|\alpha|} \mathrm{D}^{\alpha} \check{\theta}_{\mathrm{a}}\right)= \\
=\left(\delta,(-1)^{|\alpha|}(-1)^{|\alpha|} \mathrm{D}^{\alpha} \theta_{\mathrm{a}}\right)=\mathrm{D}^{\alpha} \theta(\mathrm{a}),
\end{gathered}
$$

e, portanto, $\mathrm{D}^{\alpha} \delta * \theta=\mathrm{D}^{\alpha} \theta$.

Teorema 2.24 Sejam $f \in \mathcal{P}_{\mathrm{T}}^{\prime}\left(\mathbb{R}^{n}\right)$ e $\theta \in \mathcal{P}_{\mathrm{T}}\left(\mathbb{R}^{n}\right)$. Então $f * \theta \in \mathcal{P}_{\mathrm{T}}\left(\mathbb{R}^{n}\right)$ e $\mathrm{D}^{\alpha}(\mathrm{f} * \theta)=$ $D^{\alpha} f * \theta=f * D^{\alpha} \theta$. 
DemonstraçÃo. Sejam $a \in \mathbb{R}^{n}$ e uma sequência $\left(a_{j}\right)_{j \in \mathbb{N}}$ tal que $a_{j} \longrightarrow a$. Para provar a continuidade de $f * \theta$, basta provar que $\check{\theta}_{a_{j}} \longrightarrow \check{\theta}_{a}$ em $\mathcal{P}_{\mathrm{T}}\left(\mathbb{R}^{n}\right)$, pois daí,

$$
(f * \theta)\left(a_{j}\right)=\left(f, \check{\theta}_{a_{j}}\right) \longrightarrow\left(f, \check{\theta}_{a_{j}}\right)=(f * \theta)(a) .
$$

Mostremos, então, que $\check{\theta}_{\mathrm{a}_{j}} \longrightarrow \check{\theta}_{\mathrm{a}}$ em $\mathcal{P}_{\mathrm{T}}\left(\mathbb{R}^{n}\right)$. Dado $\alpha \in \mathbb{N}^{n}$, sabemos que, devido s derivadas de $\theta$ serem limitadas, existe $C>0$, tal que

$$
\left|D^{\alpha} \check{\theta}_{a_{j}}(x)-D^{\alpha} \check{\theta}_{a}(x)\right|=\left|D^{\alpha} \theta\left(a_{j}-x\right)-D^{\alpha} \theta(a-x)\right| \leq C\left|a_{j}-a\right| .
$$

Como $a_{j} \longrightarrow a$, segue-se o resultado.

Seja $e_{i}$ o i-ésimo vetor da base canônica de $\mathbb{R}^{n}$. Dada uma sequência $\left(h_{j}\right), h_{j} \in \mathbb{R}$, $h_{j} \neq 0$ tal que $h_{j} \longrightarrow 0$, temos

$$
\begin{gathered}
{\left[(f * \theta)\left(a+h_{j} e_{i}\right)-(f * \theta)(a)\right] h_{j}^{-1}=\left[\left(f, \check{\theta}_{a+h_{j}} e_{i}\right)-\left(f, \check{\theta}_{a}\right)\right] h_{j}^{-1}=} \\
=\left[\left(f_{h_{j} e_{i}}, \check{\theta}_{a}\right)-\left(f, \check{\theta}_{a}\right)\right] h_{j}^{-1}=\left(\left(f_{h_{j} e_{i}-f}\right) h_{j}^{-1}, \check{\theta}_{a}\right) .
\end{gathered}
$$

Mostremos que $g_{j}=\left(f_{h_{j} e_{i}}-f\right) h_{j}^{-1}$ converge para $\frac{\partial}{\partial x_{i}} f$ em $\mathcal{P}_{T}^{\prime}\left(\mathbb{R}^{n}\right)$. Dada $\phi \in$ $\mathcal{P}_{\mathrm{T}}\left(\mathbb{R}^{n}\right)$, temos $\left(g_{j}, \phi\right)=\left(\left(f_{h_{j} e_{i}}-f\right) h_{j}^{-1}, \phi\right)=\left(f,\left(\phi-\phi_{-h_{j} e_{i}}\right) h_{j}^{-1}\right)$. Resta assim, mostrarmos que $\left(\phi-\phi_{-h_{j} e_{i}}\right) h_{j}^{-1} \longrightarrow-\frac{\partial}{\partial x_{i}} \phi$ em $\mathcal{P}_{\mathrm{T}}\left(\mathbb{R}^{n}\right)$. Para cada $\alpha \in \mathbb{N}^{n}$, temos que

$$
\begin{aligned}
\mid \partial^{\alpha}\left(\left(\phi-\phi_{-h_{j} e_{i}}\right) h_{j}^{-1}+\partial^{\alpha} \frac{\partial \phi}{\partial x_{i}}\right) & (x) \mid \\
& =\left|\left(\partial^{\alpha} \phi(x)-\partial^{\alpha} \phi\left(x+h_{j} e_{i}\right)-\partial^{\alpha} \frac{\partial}{\partial x_{i}} \phi(x)\right) h_{j}^{-1}\right| \longrightarrow 0,
\end{aligned}
$$

uniformemente em $\mathbb{R}^{n}$, pois $\frac{\partial}{\partial x_{i}} \partial^{\alpha} \phi$ periódica e contínua, e

$$
\left(\partial^{\alpha} \phi\left(x+h_{j} e_{i}\right)-\partial^{\alpha} \phi(x)\right) h_{j}^{-1}
$$

converge pontualmente para $\frac{\partial}{\partial x_{i}} \partial^{\alpha} \phi(x)$. Logo, $\frac{\partial}{\partial x_{i}}(f * \theta)=\frac{\partial}{\partial x_{i}} f * \theta$, e como $\frac{\partial}{\partial x_{i}} f * \theta$ contínua, temos que $f * \theta \in C^{1}\left(\mathbb{R}^{n}\right)$. Também vale que

$$
\left(\frac{\partial}{\partial x_{i}} f * \theta\right)(a)=\left(\frac{\partial}{\partial x_{i}} f, \check{\theta}_{a}\right)=\left(f,-\frac{\partial}{\partial x_{i}} \check{\theta}_{a}\right)=\left(f,\left(\frac{\partial}{\partial x_{i}} \theta\right)_{a}^{\vee}\right)=\left(f * \frac{\partial}{\partial x_{i}} \theta\right)(a) .
$$

Portanto,

$$
\frac{\partial}{\partial x_{i}}(f * \theta)=\frac{\partial}{\partial x_{i}} * \theta=f * \frac{\partial}{\partial x_{i}} \theta .
$$

Usando o princípio da indução, vemos que se $\partial^{\alpha}(f * \theta)=\partial^{\alpha} f * \theta=f * \partial^{\alpha} \theta$ é contínua, então, colocando $\beta=\alpha+e_{i}$, temos que

$$
\partial^{\beta}(f * \theta)=\frac{\partial}{\partial x_{i}} \partial^{\alpha}(f * \theta)=\frac{\partial}{\partial x_{i}}\left(\partial^{\alpha} f * \theta\right)=\partial^{\beta} f * \theta,
$$


e portanto, $\partial^{\beta}(f * \theta)$ também é contínua.

Ainda,

$$
\left(\partial^{\beta} f * \theta\right)(a)=\left(\partial^{\beta} f, \check{\theta}_{a}\right)=(-1)^{|\beta|}\left(f, \partial^{\beta} \check{\theta}_{a}\right)=\left(f,\left(\partial^{\beta} \theta\right)_{a}^{\vee}\right)=\left(f * \partial^{\beta} \theta\right)(a) .
$$

A periodicidade de $f * \theta$, se verifica através de

$$
(f * \theta)(a+T m)=\left(f, \check{\theta}_{a+T m}\right)=\left(f, \check{\theta}_{a}\right)=(f * \theta)(a) .
$$

Teorema 2.25 Se $f \in \mathcal{P}_{\mathrm{T}}^{\prime}\left(\mathbb{R}^{n}\right)$ e $\theta, \rho \in \mathcal{P}_{\mathrm{T}}\left(\mathbb{R}^{n}\right)$, ent $\tilde{a} o$

$$
(f * \theta) * \rho=f *(\theta * \rho) .
$$

Demonstração. Seja $a \in \mathbb{R}^{n}$. Para cada $k=1,2, \ldots$, tome $\varepsilon=\frac{T}{k}$, e defina

$$
S_{\varepsilon}(x)=\sum_{\substack{1 \leq m_{i} \leq k \\ 1 \leq i \leq n}} \theta(x-(a+\varepsilon m)) \rho(a+\varepsilon m) \varepsilon^{n}
$$

Note que $S_{\varepsilon}$ é uma soma de Riemann da função $g(y)=\theta(x-y) \rho(y)$ sobre o cubo $\prod_{j=1}^{n}\left[a_{j}, a_{j}+T\right]$. Mostremos que $S_{\varepsilon} \longrightarrow \theta * \rho$ Dado $\alpha \in \mathbb{N}^{n}$, existem $C>0$ e $M>0$ tais que $\left|\mathrm{D}^{\alpha} \theta(x)\right| \leq \mathrm{C},|\rho(x)| \leq \mathrm{C}$ e

$$
\left|D^{\alpha} \theta(x)-D^{\alpha} \theta(y)\right| \leq M|x-y|
$$

para todo $x, y \in \mathbb{R}^{n}$. Defina $B_{\varepsilon, i}=\prod_{j=1}^{n}\left[a_{j}+\varepsilon\left(m_{i}-1\right), a_{j}+\varepsilon m_{i}\right]$. Assim,

$$
\begin{gathered}
\left|D^{\alpha} S_{\varepsilon}(x)-D^{\alpha}(\theta * \rho)(x)\right|= \\
=\left|\sum_{\substack{1 \leq m_{i} \leq k \\
1 \leq i \leq n}} D^{\alpha} \theta(x-(a+\varepsilon m)) \rho(a+\varepsilon m) \varepsilon^{n}-\int_{\prod_{j=1}^{n}\left[a_{j}, a_{j}+T\right]} D^{\alpha} \theta(x-y) \rho(y) d y\right|= \\
=\mid \sum_{\substack{1 \leq m_{i} \leq k \\
1 \leq i \leq n}} \int_{B_{\varepsilon, i}} D^{\alpha} \theta(x-(a+\varepsilon m)) \rho(a+\varepsilon m) d y- \\
\quad-\sum_{\substack{1 \leq m_{i} \leq k \\
1 \leq i \leq n}} \int_{B_{\varepsilon, i}} D^{\alpha} \theta(x-y) \rho(y) d y \mid \leq \\
\leq \sum_{\substack{1 \leq m_{i} \leq k \\
1 \leq i \leq n}} \int_{B_{\varepsilon, i}}\left|D^{\alpha} \theta(x-(a+\varepsilon m)) \rho(a+\varepsilon m)-D^{\alpha} \theta(x-y) \rho(y)\right| d y \leq \\
\sum_{\substack{1 \leq i \leq n \\
1 \leq i \leq k}}\left(\left|D^{\alpha} \theta(x-(a+\varepsilon m))-D^{\alpha} \theta(x-y) \| \rho(a+\varepsilon m)\right|+\right. \\
\left.+D_{\varepsilon, i}^{\alpha}(x-y) \| \rho(y)-\rho(a+\varepsilon m) \mid\right) d y \leq
\end{gathered}
$$




$$
\begin{aligned}
& \leq \sum_{\substack{1 \leq m_{i} \leq k \\
1 \leq i \leq n}} M\left(|\rho(a+\varepsilon m)| \int_{B_{\varepsilon, i}}|y-(a+\varepsilon m)| d y\right. \\
& \left.\quad+\int_{B_{\varepsilon, i}}\left|D^{\alpha} \theta(x-y)\right||y-(a+\varepsilon m) d y|\right) \leq \\
& \leq \mathrm{CM} \sum_{\substack{1 \leq m_{i} \leq k \\
1 \leq i \leq n}}\left(\sqrt{n} \varepsilon \int_{B_{\varepsilon, i}} d y+\sqrt{n} \varepsilon \int_{B_{\varepsilon, i}} d y\right)= \\
& =2 \mathrm{CM} \varepsilon^{n+1} \sum_{\substack{1 \leq m_{i} \leq k \\
1 \leq i \leq n}} 1=2 \mathrm{CMk}^{n} \varepsilon^{n+1}=\frac{2 \mathrm{CMT}^{n+1}}{k} \longrightarrow 0,
\end{aligned}
$$

quando $\mathrm{k} \longrightarrow 0$.

Como $S_{\varepsilon}(x+T m)=S_{\varepsilon}(x)$, temos que $S_{\varepsilon} \longrightarrow \theta * \rho$ em $\mathcal{P}_{\mathrm{T}}\left(\mathbb{R}^{n}\right)$ e, portanto, $\left(S_{\varepsilon}\right)_{x}=$ $(\theta * \rho)_{\bar{x}}^{\sim}$. Logo

$$
\begin{gathered}
(f *(\theta * \rho))(x)=\lim _{\varepsilon \longrightarrow 0}\left(f * S_{\varepsilon}\right)(x)= \\
=\lim _{\varepsilon \longrightarrow 0} \varepsilon^{n} \sum_{\substack{1 \leq m_{i} \leq k \\
1 \leq i \leq n}}(f * \theta)(x-(a+\varepsilon m)) \rho(a+\varepsilon m)= \\
=\int_{\prod_{j=1}^{n}\left[a_{j}, a_{j}+T\right]}(f * \theta)(x-y) \rho(y) d y=((f * \theta) * \rho)(x)
\end{gathered}
$$

Teorema 2.26 Seja $\mathrm{F}: \mathcal{P}_{\mathrm{T}}\left(\mathbb{R}^{n}\right) \longrightarrow \mathcal{P}_{\mathrm{T}}\left(\mathbb{R}^{n}\right)$ um operador linear continuo tal que para todo $\mathrm{h} \in \mathbb{R}^{\mathrm{n}}$ e toda $\theta \in \mathcal{P}_{\mathrm{T}}\left(\mathbb{R}^{\mathfrak{n}}\right)$, vale

$$
\mathrm{F}(\theta)(x-h)=F\left(\theta_{h}\right)(x) .
$$

Então existe uma única $f \in \mathcal{P}_{\mathrm{T}}^{\prime}\left(\mathbb{R}^{n}\right)$ tal que $\mathrm{F}(\theta)=\mathrm{f} * \theta$, para toda $\theta \in \mathcal{P}_{\mathrm{T}}\left(\mathbb{R}^{n}\right)$.

Demonstração. Defina

$$
\begin{aligned}
f: \mathcal{P}_{\mathrm{T}}\left(\mathbb{R}^{n}\right) & \longrightarrow c \\
\theta & \longmapsto(\mathrm{F}(\check{\theta}))(0) .
\end{aligned}
$$

A linearidade de $f$ imediata.

Continuidade: Se $\theta_{j} \longrightarrow 0$ em $\mathcal{P}_{\mathrm{T}}\left(\mathbb{R}^{\mathfrak{n}}\right)$, então, pela continuidade de $F, F\left(\check{\theta}_{j}\right) \longrightarrow 0$ em $\mathcal{P}_{\mathrm{T}}\left(\mathbb{R}^{n}\right)$, e consequentemente, $\left(\mathrm{F}\left(\check{\theta}_{j}\right)\right)(0) \longrightarrow 0$ em $\mathbb{C}$. Logo, $f \in \mathcal{P}_{\mathrm{T}}^{\prime}\left(\mathbb{R}^{\mathrm{n}}\right)$.

Note agora, que para todo $h \in \mathbb{R}^{n}$, vale

$$
(F *(\theta))(h)=(F(\theta))_{-h}(0)=\left(F\left(\theta_{-h}\right)\right)(0)=\left(f, \check{\theta}_{h}\right)=\left(f, \check{\theta}_{h}\right)=(f * \theta)(h) .
$$

Unicidade: Sejam $f, g \in \mathcal{P}_{\top}^{\prime}\left(\mathbb{R}^{n}\right)$ tais que $F(\theta)=f * \theta=g * \theta$, para toda $\theta \in \mathcal{P}_{\mathrm{T}}\left(\mathbb{R}^{n}\right)$. Então

$$
(f, \theta)=(f * \check{\theta})(0)=(g * \check{\theta})(0)=(g, \theta),
$$

e com isso, $f=g$. 
Definição 2.27 Sejam $\mathrm{f}, \mathrm{g} \in \mathcal{P}_{\mathrm{T}}^{\prime}\left(\mathbb{R}^{\mathrm{n}}\right)$. Definimos $\mathrm{h}=\mathrm{f} * \mathrm{~g}$ como sendo a única distribuição periódica tal que

$$
\mathrm{f} *(\mathrm{~g} * \theta)=\mathrm{h} * \theta,
$$

para todo $\theta \in \mathcal{P}_{\mathrm{T}}\left(\mathbb{R}^{\mathrm{n}}\right)$.

Chamamos $\mathrm{h}$ de $\mathrm{T}$-convolução de f por $\mathrm{g}$.

Para verificar que a definição é possível, mostremos que

$$
\begin{aligned}
\mathrm{F}: \mathcal{P}_{\mathrm{T}}\left(\mathbb{R}^{\mathrm{n}}\right) & \longrightarrow \mathcal{P}_{\mathrm{T}}\left(\mathbb{R}^{\mathrm{n}}\right) \\
\theta & \longmapsto f *(\mathrm{~g} * \theta)
\end{aligned}
$$

é linear, contínuo e comuta com translações.

Linearidade: Se $\theta, \rho \in \mathcal{P}_{\mathrm{T}}\left(\mathbb{R}^{n}\right)$ e $\lambda \in \mathbb{C}$, então

$$
\begin{gathered}
F(\theta+\rho)=f *(g *(\theta+\rho))=f *(g * \theta+g * \rho)=f *(g * \theta)+f *(g * \rho)=F(\theta)+F(\rho) . \\
F(\lambda \theta)=f *(g *(\lambda \theta))=f *(\lambda(g * \theta))=\lambda f *(g * \theta)=\lambda F(\theta) .
\end{gathered}
$$

Continuidade: Seja $\theta_{j} \longrightarrow 0$, em $\mathcal{P}_{\mathrm{T}}\left(\mathbb{R}^{n}\right)$. Dado $\alpha \in \mathbb{N}^{n}$, existem $C_{1}, C_{2}>0$, $\mathrm{m}_{1}, \mathrm{~m}_{2} \in \mathbb{N}$, tais que

$$
\begin{gathered}
\left|D^{\alpha}\left(F\left(\theta_{j}\right)\right)(a)\right|=\left|f * D^{\alpha}\left(g * \theta_{j}\right)(a)\right|=\left|\left(f,\left(D^{\alpha}\left(g * \theta_{j}\right)\right)_{a}\right)\right| \leq \\
\leq C_{1} \sum_{|\beta| \leq m_{1}} \sup _{x}\left\{\left|D^{\beta}\left(D^{\alpha}\left(g * \theta_{j}\right)\right)_{a}\right|\right\}=C_{1} \sum_{|\beta| \leq m_{1}} \sup _{x}\left\{\left|\left(D^{\beta+\alpha}\left(g * \theta_{j}\right)\right)_{a}\right|\right\}= \\
=C_{1} \sum_{|\beta| \leq m_{1}} \sup _{x}\left\{\left|\left(g * D^{\beta+\alpha} \theta_{j}\right)(a-x)\right|\right\}=C_{1} \sum_{|\beta| \leq m_{1}} \sup _{x}\left\{\left|\left(g,\left(D^{\beta+\alpha} \theta_{j}\right)\right)_{a}-x\right|\right\} \leq \\
\leq C_{1} C_{2} \sum_{|\beta| \leq m_{1}} \sup _{x}\left\{\sum_{|\gamma| \leq m_{2}} \sup _{y}\left\{\left|D^{\gamma}\left(D^{\beta+\alpha} \theta_{j}\right)_{a-x}\right|\right\}\right\}= \\
=C_{1} C_{2} \sum_{|\beta| \leq m_{1}} \sup _{x}\left\{\sum_{|\gamma| \leq m_{2}} \sup _{z}\left\{\left|\left(D^{\gamma \beta+\alpha} \theta_{j}(z-x)\right)\right|\right\}\right\} \longrightarrow 0,
\end{gathered}
$$

uniformemente e, portanto, F é contínua.

Comutatividade: Sejam $h \in \mathbb{R}^{n}$ e $\theta \in \mathcal{P}_{\mathrm{T}}\left(\mathbb{R}^{n}\right)$. Note que

$$
\begin{gathered}
(F \theta)(a-h)=(f *(g * \theta))(a-h)=\left(f,(g * \theta)_{a}-h\right)=\left(f,((g * \theta)(x-h))_{a}\right)= \\
=(f *(g * \theta(x-h)))(a)=(f *(g * \theta(x-h)))(a)=F(\theta(x-h))(a) .
\end{gathered}
$$

Justificativa da igualdade $(g * \theta)(x-h)=g *(\theta(x-h))$ :

$$
(g * \theta)(a-h)=\left(g, \check{\theta}_{a-h}\right)=(g * \theta(x-h))(a) .
$$


Antes de mostrarmos algumas propriedades da T-convolução, vejamos o seguinte lema:

Lema 2.28 Considere $\phi \in \mathrm{C}_{\mathrm{c}}^{\infty}\left(\mathbb{R}^{n}\right)$ tal que $\int_{\mathbb{R}^{n}} \phi=1, \phi \geq 0$ e $S(\phi) \subset[0, T]^{n}$. Seja também $\theta_{\varepsilon}(x)=\varepsilon^{-n} \sum_{m \in \mathbb{Z}^{n}} \phi\left(\frac{x-T m}{\varepsilon}\right)$, onde $0<\varepsilon<1$. Observe que $\theta_{\varepsilon} \in \mathcal{P}_{T}\left(\mathbb{R}^{n}\right)$.

Então, se $\psi \in \mathcal{P}_{\mathrm{T}}\left(\mathbb{R}^{n}\right), \psi * \theta_{\varepsilon} \longrightarrow \psi$ em $\mathcal{P}_{\mathrm{T}}\left(\mathbb{R}^{n}\right)$.

DemonstraçÃo. Seja $\alpha \in \mathbb{N}^{n}$, segue que

$$
D^{\alpha}\left(\psi * \theta_{\varepsilon}\right)(a)=\int_{[0, T]^{n}} D^{\alpha} \psi(a-x) \theta_{\varepsilon}(x) d x=\varepsilon^{-n} \int_{[0, T]^{n}} D^{\alpha} \psi(a-x) \phi\left(\frac{x}{\varepsilon}\right) d x,
$$

pois, se $x \in[0, T]^{n}$, então para todo $m=\left(m_{1}, \ldots, m_{n}\right) \in \mathbb{Z}^{n}, m \neq 0$, existe $m_{i} \neq 0$, tal que

$$
-\frac{T m_{i}}{\varepsilon} \leq \frac{x_{i}-T m_{i}}{\varepsilon} \leq \frac{T\left(1-m_{i}\right)}{\varepsilon}
$$

Se $m_{i} \geq 1$, então $\frac{x_{i}-T m_{i}}{\varepsilon} \leq 0$, e então $\phi\left(\frac{x-T m}{\varepsilon}\right)=0$. Se $m_{i} \leq-1$, então $-\frac{T m_{i}}{\varepsilon} \geq \frac{I}{\varepsilon} \geq T$ e $\phi\left(\frac{x-T m}{\varepsilon}\right)=0$.

Deste modo, $\theta_{\varepsilon}(x)=\varepsilon^{-n} \phi\left(\frac{x}{\varepsilon}\right)$, se $x \in[0, T]^{n}$. Também vale que

$$
\begin{gathered}
\varepsilon^{-n} \int_{[0, T]^{n}} D^{\alpha} \psi(a-x) \phi\left(\frac{x}{\varepsilon}\right) d x=\int_{\left[0, \frac{T}{\varepsilon}\right]^{n}} D^{\alpha} \psi(a-\varepsilon y) \phi(y) d y= \\
\int_{[0, T]^{n}} D^{\alpha} \psi(a-\varepsilon y) \phi(y) d y,
\end{gathered}
$$

pois $S(\phi) \subset[0, T]^{\mathrm{n}} \subset\left[0, \frac{\mathrm{T}}{\varepsilon}\right]^{\mathrm{n}}$. Assim,

$$
\begin{aligned}
& \left|D^{\alpha} \psi(a)-\left(D^{\alpha} \psi * \theta_{\varepsilon}\right)(a)\right|=\left|\int_{[0, T]^{n}}\left(D^{\alpha} \psi(a)-D^{\alpha} \psi(a-\varepsilon x)\right) \phi(x) d x\right| \leq \\
& \leq \int_{[0, T]^{n}} \phi(x) d x \sup \left\{\left|D^{\alpha} \psi(a)-D^{\alpha} \psi(a-\varepsilon x)\right| \leq C \sup _{x \in[0, T]^{n}}\{|\varepsilon x|\}=\sqrt{n} C T \varepsilon .\right.
\end{aligned}
$$

Logo, $\psi * \theta_{\varepsilon} \longrightarrow \psi$ em $\mathcal{P}_{\mathrm{T}}\left(\mathbb{R}^{\mathrm{n}}\right)$.

Lema 2.29 Sejam $f, g \in \mathcal{P}_{\mathrm{T}}^{\prime}\left(\mathbb{R}^{n}\right)$. Então $\mathrm{f}=\mathrm{g}$ se e somente se $\mathrm{f} * \theta=\mathrm{g} * \theta$, para toda $\theta \in \mathcal{P}_{\mathrm{T}}\left(\mathbb{R}^{n}\right)$. 
DEMONSTRAÇÃo. Se $f=g$ então

$$
(f * \theta)(a)=\left(f, \check{\theta}_{a}\right)=\left(g, \check{\theta}_{a}\right)=(g * \theta) .
$$

Se $g * \theta=f * \theta$ então

$$
(f, \theta)=(f * \check{\theta})(0)=(g * \check{\theta})(0)=(g, \theta) .
$$

Teorema 2.30 Se $\mathrm{f}, \mathrm{g}, \mathrm{h} \in \mathcal{P}_{\mathrm{T}}^{\prime}\left(\mathbb{R}^{\mathrm{n}}\right)$ então

1. $(\mathrm{f} * \mathrm{~g}) * \mathrm{~h}=\mathrm{f} *(\mathrm{~g} * \mathrm{~h})$.

2. $f * g=g * f$.

Demonstração. Seja $\theta \in \mathcal{P}_{\mathrm{T}}\left(\mathbb{R}^{n}\right)$. Temos

$((\mathrm{f} * \mathrm{~g}) * \mathrm{~h})(\theta)=(\mathrm{f} * \mathrm{~g}) *(\mathrm{~h} * \theta)=\mathrm{f} *(\mathrm{~g} *(\mathrm{~h} * \theta))=\mathrm{f} *((\mathrm{~g} * \mathrm{~h}) * \theta)=(\mathrm{f} *(\mathrm{~g} * \mathrm{~h})) * \theta$.

Usando o lema anterior, concluímos que $(\mathrm{f} * \mathrm{~g}) * \mathrm{~h}=\mathrm{f} *(\mathrm{~g} * \mathrm{~h})$.

Para provar 2, seja $\theta_{\varepsilon}$ como no lema 2.28. Provemos primeiramente que $\theta_{\varepsilon} * \theta=$ $\theta * \theta_{\varepsilon}$.

$$
\begin{aligned}
\left(\theta_{\varepsilon} * \theta\right)(a)=\int_{[0, T]^{n}} \theta_{\varepsilon}(a-x) \theta(x) d x & = \\
& =\int_{\prod_{j=1}^{n}\left[a_{j}, a_{j}+T\right]} \theta_{\varepsilon}(y) \theta(a-y) d y=\left(\theta * \theta_{\varepsilon}\right)(a) .
\end{aligned}
$$

Agora,

$$
\begin{gathered}
(\mathrm{f} * \mathrm{~g}) *\left(\theta * \theta_{\varepsilon}\right)=\mathrm{f} *\left(\mathrm{~g} *\left(\theta * \theta_{\varepsilon}\right)\right)= \\
=\mathrm{f} *\left((\mathrm{~g} * \theta) * \theta_{\varepsilon}\right)=\mathrm{f} *\left(\theta_{\varepsilon} *(\mathrm{~g} * \theta)\right)=\left(\mathrm{f} * \theta_{\varepsilon}\right) *(\mathrm{~g} * \theta) .
\end{gathered}
$$

Por outro lado,

$$
\begin{gathered}
(\mathrm{g} * \mathrm{f}) *\left(\theta * \theta_{\varepsilon}\right)=(\mathrm{g} * \mathrm{f}) *\left(\theta_{\varepsilon} * \theta\right)= \\
=g *\left(\mathrm{f} *\left(\theta_{\varepsilon} * \theta\right)\right)=\mathrm{g} *\left(\left(\mathrm{f} * \theta_{\varepsilon}\right) * \theta\right)= \\
=g *\left(\theta *\left(\mathrm{f} * \theta_{\varepsilon}\right)\right)=(\mathrm{g} * \theta) *\left(\mathrm{f} * \theta_{\varepsilon}\right)=\left(\mathrm{f} * \theta_{\varepsilon}\right) *(\mathrm{~g} * \theta) .
\end{gathered}
$$

Comparando,

$$
(\mathbf{f} * \mathbf{g}) *\left(\theta * \theta_{\varepsilon}\right)=(\mathrm{g} * \mathbf{f}) *\left(\theta * \theta_{\varepsilon}\right) .
$$

Quando $\varepsilon \longrightarrow 0$, pelo lema 2.28, $\theta * \theta_{\varepsilon} \longrightarrow \theta$ em $\mathcal{P}_{\mathrm{T}}\left(\mathbb{R}^{n}\right)$. Logo $(\mathrm{f} * \mathrm{~g}) * \theta=(\mathrm{g} * \mathrm{f}) * \theta$ e pelo lema $4.8, f * g=g * f$. 
Teorema 2.31 Sejam $f, g \in \mathcal{P}_{\mathrm{T}}^{\prime}\left(\mathbb{R}^{n}\right)$ e $a \in \mathbb{R}^{n}$. Então

1. $(f * g)_{a}=f_{a} * g=f * g_{a}$.

2. $D^{\alpha}(f * g)=D^{\alpha} f * g=f * D^{\alpha} g, \forall \alpha \in \mathbb{N}^{n}$.

Demonstração. Mostremos primeiramente que se $\theta \in \mathcal{P}_{\mathrm{T}}\left(\mathbb{R}^{\mathrm{n}}\right)$ então $f_{\mathrm{a}} * \theta=f * \theta_{-a}$. Observe que isto não contradiz 1 , pois se $T_{\theta}$ é a distribuição periódica oriunda da função $\theta$, então $T_{\theta_{-a}}=\left(T_{\theta}\right)_{a}$.

Temos

$$
\left(f_{a} * \theta\right)(x)=\left(f_{a}, \check{\theta}_{x}\right)=\left(f,\left(\check{\theta}_{x}\right)_{-a}\right)=\left(f, \check{\theta}_{x+a}\right)=\left(f,\left(\theta_{-a}\right)_{x}\right)=\left(f * \theta_{-a}\right)(x) .
$$

Assim,

$(\mathrm{f} * \mathrm{~g})_{\mathrm{a}} * \theta=(\mathrm{f} * \mathrm{~g}) * \theta_{-a}=\mathrm{f} *\left(\mathrm{~g} * \theta_{-a}\right)=\mathrm{f} *\left(\mathrm{~g} * \theta_{-a}\right)=\mathrm{f} *\left(g_{\mathrm{a}} * \theta\right)=\left(\mathrm{f} * \mathrm{~g}_{\mathrm{a}}\right) * \theta$.

Portanto, $(\mathrm{f} * \mathrm{~g})_{\mathrm{a}}=\mathrm{f} * \mathrm{~g}_{\mathrm{a}}$. Também vale que

$$
\begin{gathered}
(f * g)_{a} * \theta=(g * f)_{a} * \theta=(g * f) * \theta_{-a}=g *\left(f * \theta_{-a}\right)= \\
=g *\left(f_{a} * \theta\right)=\left(g * f_{a}\right) * \theta=\left(f_{a} * g\right) * \theta .
\end{gathered}
$$

Mostremos agora 2.

$$
\begin{gathered}
\left(D^{\alpha}(f * g) * \theta\right)(a)=\left(D^{\alpha}(f * g), \check{\theta}_{a}\right)=\left(f * g,(-1)^{|\alpha|} D^{\alpha}\left(\check{\theta}_{a}\right)\right)= \\
=\left(f * g,\left(D^{\alpha} \theta\right) \check{a}\right)=\left((f * g) * D^{\alpha} \theta\right)(a)=\left(f *\left(g * D^{\alpha} \theta\right)\right)(a)= \\
\left(f *\left(g,\left(D^{\alpha} \theta\right)_{x}\right)\right)(a)=(-1)^{|\alpha|}\left(f *\left(g, D^{\alpha}\left(\check{\theta}_{x}\right)\right)\right)(a)=\left(\left(f * D^{\alpha} g\right) * \theta\right)(a) .
\end{gathered}
$$

Portanto, $D^{\alpha}(f * g)=f * D^{\alpha} g$. Por comutatividade, obtemos a outra igualdade.

Exemplo 2.32 Sejam $f \in \mathcal{P}_{\mathrm{T}}^{\prime}\left(\mathbb{R}^{\mathrm{n}}\right)$ e $\theta \in \mathcal{P}_{\mathrm{T}}\left(\mathbb{R}^{\mathrm{n}}\right)$. Temos

$$
(f * \delta, \theta)=(f * \delta * \check{\theta})(0)=(f * \check{\theta})(0)=(f, \theta) .
$$

$\log o, f * \delta=f$

Exemplo 2.33 Sejam $\alpha \in \mathbb{N}^{n}$ ef $\in \mathcal{P}_{\mathrm{T}}^{\prime}\left(\mathbb{R}^{n}\right)$. Temos

$$
D^{\alpha} f=D^{\alpha}(f * \delta)=D^{\alpha} f * \delta=f * D^{\alpha} \delta
$$


Exemplo 2.34 Seja $\mathrm{P}(\mathrm{D})=\sum_{|\alpha| \leq m} \mathrm{c}_{\alpha} \mathrm{D}^{\alpha}$ um operador diferencial parcial linear, a coeficientes constantes. Se $u \in \mathcal{P}_{\mathrm{T}}^{\prime}\left(\mathbb{R}^{\mathfrak{n}}\right)$ então

$$
\begin{gathered}
P(D) u=\sum_{|\alpha| \leq m} c_{\alpha} D^{\alpha} u=\sum_{|\alpha| \leq m} c_{\alpha} u * D^{\alpha} \delta=\sum_{|\alpha| \leq m} u *\left(c_{\alpha} D^{\alpha} \delta\right)= \\
=\mathfrak{u} *\left(\sum_{|\alpha| \leq m} c_{\alpha} D^{\alpha}\right)=\mathfrak{u} *(P(D) \delta) .
\end{gathered}
$$

Assim, toda equação diferencial parcial linear com coeficientes constantes, $\mathrm{P}(\mathrm{D}) \mathrm{u}=\mathrm{f}$, onde $\mathrm{u}, \mathrm{f} \in \mathcal{P}_{\mathrm{T}}^{\prime}\left(\mathbb{R}^{\mathrm{n}}\right)$ pode ser expressa como uma equação de $\mathrm{T}$ convolução $\mathrm{u} * \mathrm{u}_{0}=\mathrm{f}$, onde $\mathrm{u}_{0}=\mathrm{P}(\mathrm{D}) \delta$.

Teorema 2.35 Seja

$$
\begin{aligned}
& C: \mathcal{P}_{\mathrm{T}}^{\prime}\left(\mathbb{R}^{n}\right) \times \mathcal{P}_{\mathrm{T}}^{\prime}\left(\mathbb{R}^{n}\right) \longrightarrow \mathcal{P}_{\mathrm{T}}^{\prime}\left(\mathbb{R}^{\mathfrak{n}}\right) \\
&(\mathrm{f}, \mathrm{g}) \longmapsto \\
& \mathrm{f} * \mathrm{~g}
\end{aligned} .
$$

Então $C$ é sequencialmente contínuo em cada variável.

Demonstração. Como $C$ é simétrico, basta demonstrar em uma variável. Como $C$ é linear, basta verificar que se $f_{j} \longrightarrow 0$ em $\mathcal{P}_{T}^{\prime}\left(\mathbb{R}^{n}\right)$ então $C\left(f_{j}, g\right)=f_{j} * g \longrightarrow 0$ em $\mathcal{P}_{T}^{\prime}\left(\mathbb{R}^{n}\right)$.

Seja $\theta \in \mathcal{P}_{\mathrm{T}}\left(\mathbb{R}^{n}\right)$, então $\left(C\left(f_{j}, g\right), \theta\right)=\left(f_{j} * g, \theta\right)=\left(f_{j} *(g * \check{\theta})\right)(0)$. Como $g * \check{\theta} \in$ $\mathcal{P}_{\mathrm{T}}\left(\mathbb{R}^{n}\right)$, e $f_{j} \longrightarrow 0$, em $\mathcal{P}_{\mathrm{T}}^{\prime}\left(\mathbb{R}^{n}\right)$, segue-se que $\left(f_{j} *(g * \check{\theta})\right)(0)=\left(f_{j},(g * \check{\theta})^{\sim}\right) \longrightarrow 0$.

\subsection{A Álgebra $\left(\mathcal{P}_{\mathrm{T}}^{\prime}\left(\mathbb{R}^{n}\right), *,+\right)$}

O espaço vetorial $\mathcal{P}_{\mathrm{T}}^{\prime}\left(\mathbb{R}^{n}\right)$, munido das operações T-convolução e adição, possui uma estrutura de álgebra comutativa, com elemento neutro $\delta$, em relação à T-convolução. Designaremos esta álgebra por álgebra da T-convolução.

O próximo teorema nos dará uma condição necessária e suficiente para que possamos encontrar $u \in \mathcal{P}_{\top}^{\prime}\left(\mathbb{R}^{n}\right)$, tal que $f * u=g$, onde $f, g$ são distribuições periódicas conhecidas anteriormente.

Antes disso, definamos o inverso (segundo a T-convolução), de um elemento de $\mathcal{P}_{\mathrm{T}}^{\prime}\left(\mathbb{R}^{\mathrm{n}}\right)$.

Definição 2.36 Dizemos que $f \in \mathcal{P}_{\mathrm{T}}^{\prime}\left(\mathbb{R}^{n}\right)$ é invertivel, se existir $\mathrm{g} \in \mathcal{P}_{\mathrm{T}}^{\prime}\left(\mathbb{R}^{n}\right)$, tal $q u e f * g=g * f=\delta$. Uma $g$ desse tipo é dita inversa de $\mathrm{f}$.

Teorema 2.37 Se existir inversa de uma distribuição periódica ela é única. 
Demonstração. Sejam $g$ e $h$ inversas de $f$. Então $g=g * \delta=g *(f * h)=$ $(\mathrm{g} * \mathrm{f}) * \mathrm{~h}=\delta * \mathrm{~h}=\mathrm{h}$.

Denotemos por $f^{-1}$ o inverso de $f$, quando existir. Nem todos elementos de $\mathcal{P}_{T}^{\prime}\left(\mathbb{R}^{n}\right)$ possuem inverso, como é o caso de qualquer $\theta \in \mathcal{P}_{T}\left(\mathbb{R}^{n}\right)$. Pois, como visto no teorema 2.24, página $28, f * \theta \in \mathcal{P}_{\mathrm{T}}\left(\mathbb{R}^{n}\right)$, para toda $f \in \mathcal{P}_{\mathrm{T}}^{\prime}\left(\mathbb{R}^{\mathrm{n}}\right)$.

Teorema 2.38 Seja $f \in \mathcal{P}_{\top}^{\prime}\left(\mathbb{R}^{n}\right)$. Uma condição necessária e suficiente para que a equação $\mathrm{f} * \mathrm{u}=\mathrm{g}$ tenha, para cada $\mathrm{g} \in \mathcal{P}_{\mathrm{T}}^{\prime}\left(\mathbb{R}^{\mathrm{n}}\right)$, uma solução em $\mathcal{P}_{\mathrm{T}}^{\prime}\left(\mathbb{R}^{\mathrm{n}}\right)$ é que exista $\mathrm{f}^{-1}$. Neste caso, a solução é única e dada por $\mathrm{f}^{-1} * \mathrm{~g}$.

DemonstraçÃo. Necessidade: Existe $u \in \mathcal{P}_{T}^{\prime}\left(\mathbb{R}^{\mathfrak{n}}\right)$ tal que $f * u=\delta$. Logo, $u=f^{-1}$.

Suficiência: Suponha que exista $f^{-1}$. Tome $u=f^{-1} * g$. Então $f * u=f *\left(f^{-1} * g\right)=$ $\left(f * f^{-1}\right) * g=\delta * g=g$. Se $f * v=g$ então

$$
\begin{gathered}
\mathrm{f}^{-1} *(\mathrm{f} * v)=\mathrm{f}^{-1} * \mathrm{~g} \\
\left(\mathrm{f}^{-1} * \mathrm{f}\right) * v=\mathrm{f}^{-1} * \mathrm{~g} \\
v=\mathrm{f}^{-1} * \mathrm{~g} .
\end{gathered}
$$




\section{Capítulo 3}

\section{Séries de Fourier em $\mathcal{P}_{\mathrm{T}}\left(\mathbb{R}^{\mathfrak{n}}\right)$ e $\mathcal{P}_{\top}^{\prime}\left(\mathbb{R}^{\mathfrak{n}}\right)$}

Apresentamos nesse capítulo a noção de série de Fourier para uma distribuição periódica. Assim como em $\mathcal{P}_{\mathrm{T}}\left(\mathbb{R}^{n}\right)$, toda distribuição periódica em $\mathcal{P}_{\mathrm{T}}^{\prime}\left(\mathbb{R}^{n}\right)$ possui série de Fourier que converge para a mesma. $O$ espaço das sequências formadas pelos coeficientes de Fourier dos elementos de $\mathcal{P}_{\mathrm{T}}^{\prime}\left(\mathbb{R}^{n}\right)$ pode ser identificado com $\mathcal{P}_{\mathrm{T}}^{\prime}\left(\mathbb{R}^{n}\right)$. O mesmo vale para $\mathcal{P}_{T}\left(\mathbb{R}^{\mathfrak{n}}\right)$.

No que diz respeito a séries de Fourier em $\mathcal{P}_{T}^{\prime}\left(\mathbb{R}^{n}\right)$ vemos que o conjunto das funções $\left\{e_{m} ; m \in \mathbb{Z}^{n}\right\}$ é uma base, no sentido de Schauder para o espaço vetorial $\mathcal{P}_{T}^{\prime}\left(\mathbb{R}^{n}\right)$. Considerando a convergência de séries em $\mathcal{P}_{T}^{\prime}\left(\mathbb{R}^{\mathfrak{n}}\right)$, todo elemento de $\mathcal{P}_{\mathrm{T}}^{\prime}\left(\mathbb{R}^{\mathfrak{n}}\right)$ é uma série trigonométrica, com coeficientes de crescimento lento. Toda série trigonométrica com coeficientes de crescimento lento, converge em $\mathcal{P}_{\mathrm{T}}^{\prime}\left(\mathbb{R}^{n}\right)$. Essa relação entre sequências e distribuições é um isomorfismo. O espaço das sequências de decrescimento rápido, está contido no espaço das sequências de crescimento lento, o que simboliza o fato de enxergarmos uma função como distribuição.

Por fim apresentamos um conceito muito importante para a obtenção do resultado central desta dissertação, a noção de série parcial de Fourier de funções teste, e de distribuições, bem como propriedades do decaimento dos coeficientes parciais de Fourier.

\subsection{Série de Fourier em $\mathcal{P}_{\mathrm{T}}\left(\mathbb{R}^{\mathfrak{n}}\right)$}

Definição 3.1 Uma sequência numérica $\left(\mathrm{c}_{\mathrm{m}}\right)_{\mathrm{m} \in \mathbb{Z}^{\mathfrak{n}}}$ é dita rapidamente decrescente se para cada $k \in \mathbb{N}$, existir $C>0$ tal que $\left|c_{m}\right| \leq \frac{C}{|m|^{k}}$, para todo $m \in \mathbb{Z}^{n}$, $\mathrm{m} \neq 0$.

Equivalentemente, $\left(c_{\mathfrak{m}}\right)_{\mathfrak{m} \in \mathbb{Z}^{n}}$ é rapidamente decrescente, se para cada $k \in \mathbb{N}$, existir $C>0$, tal que $\left|c_{m}\right| \leq \frac{c}{(1+|m|)^{k}}$ para todo $m \in \mathbb{Z}^{n}$. Pois, se para cada $k \in \mathbb{Z}^{+}$existir $C>0$ tal que 


$$
\left|c_{m}\right| \leq \frac{C}{|m|^{k}}, \quad m \neq 0, \quad m \in \mathbb{Z}^{n},
$$

então, se $m \neq 0$, vale que $1+|m| \leq 2|m|$ e

$$
\left|c_{m}\right| \leq \frac{C}{|m|^{k}} \leq \frac{2^{k} C}{(1+|m|)^{k}}
$$

Colocando $M=2^{k} C$, obtemos $\left|c_{m}\right| \leq \frac{M}{(1+|m|)^{k}}$, para todo $m \in \mathbb{Z}^{n}$.

Agora, se para cada $k \in \mathbb{N}$ existir $C>0$ tal que $\left|c_{m}\right| \leq \frac{c}{(1+|m|)^{k}}, m \in \mathbb{Z}^{n}$, então, como para $\mathrm{m} \neq 0$, vale $\frac{1}{(1+|\mathfrak{m}|)^{k}} \leq \frac{1}{|\mathfrak{m}|^{k}}$, chegamos a

$$
\left|c_{m}\right| \leq \frac{C}{|m|^{k}}
$$

para $m \in \mathbb{Z}^{n}, m \neq 0$.

O espaço das sequências de decrescimento rápido será denotado por $s\left(\mathbb{Z}^{n} ; \mathbb{C}\right)$.

Teorema 3.2 Seja $\left(c_{m}\right)_{m \in \mathbb{Z}^{n}}$ uma sequência rapidamente decrescente. Então a função

$$
f=\sum_{m \in \mathbb{Z}^{n}} c_{m} e_{m}
$$

pertence $a \mathcal{P}_{T}\left(\mathbb{R}^{n}\right)$, com $e_{m}(x)=e^{i \omega m x}=e^{i \omega\left(m_{1} x_{1}+\cdots+m_{n} x_{n}\right)}, \omega=2 \pi T^{-1}, x \in \mathbb{R}^{n}$.

DemonstraçÃo. Considere $s_{p}(x)=\sum_{|m| \leq p} c_{m} e_{m}(x)$.

Como $\left(c_{m}\right)_{m \in \mathbb{Z}^{n}}$ é de decrescimento rápido, dado $n \in \mathbb{N}$, existe $M_{1}>0$ tal que

$$
\left|c_{m} e_{m}(x)\right|=\left|c_{m}\right|<\frac{M_{1}}{(1+|m|)^{n+1}},
$$

para todo $m \in \mathbb{Z}^{n}$. Pelo critério de Weierstrass, $s_{p}$ é uniformemente convergente e segue que $f(x)=\sum_{m \in \mathbb{Z}^{n}} c_{m} e_{m}(x)$ é contínua. Note que $f$ é periódica.

Agora,

$$
\frac{\partial}{\partial x_{j}} s_{p}(x)=\sum_{|m| \leq p} i \omega m_{j} c_{m} e^{i \omega m x} .
$$

Novamente, existe $M_{2}>0$ tal que

$$
\begin{aligned}
& \left|i \omega m_{j} c_{m} e^{i \omega m x}\right|=\omega\left|m_{j}\right|\left|c_{m}\right| \leq \omega(1+|m|)\left|c_{m}\right| \leq \\
& \leq(1+|m|) M_{2}(1+|m|)^{-n-2}=M_{2}(1+|m|)^{-n-1} .
\end{aligned}
$$

Assim, $g_{j}(x)=\lim _{p} \longrightarrow \infty \frac{\partial}{\partial x_{j}} s_{p}(x)$ é contínua (a convergência é uniforme).

Sejam $x, h \in \mathbb{R}^{n}$ e $\gamma:[0,1] \longrightarrow \mathbb{R}^{n}$ dada por $\gamma(t)=x+$ th. 


\section{Então}

$$
\begin{gathered}
s_{\mathfrak{p}}(x+h)-s_{p}(x)=s_{p}(\gamma(1))-s_{p}(\gamma(0))=\int_{0}^{1}\left(s_{p} \circ \gamma\right)^{\prime}(t) d t= \\
=\int_{0}^{1}\left\langle\operatorname{grad} s_{p}(\gamma(t)), \gamma^{\prime}(t)\right\rangle d t=\int_{0}^{1}\left\langle\operatorname{grad} s_{p}(x+t h), h\right\rangle d t
\end{gathered}
$$

onde $\langle$,$\rangle denota o produto interno em \mathbb{R}^{n}$. Defina $g=\left(g_{1}, \ldots, g_{n}\right)$. Assim, quando $p \longrightarrow \infty$, vem que

$$
\begin{gathered}
f(x+h)-f(x)=\int_{0}^{1}\langle g(x+t h, h)\rangle d t=\sum_{j=1}^{n} h_{j} \int_{0}^{1} g_{j}(x+t h) d t= \\
=\sum_{j=1}^{n} h_{j} g_{j}(x)+\sum_{j=1}^{n} h_{j} \int_{0}^{1}\left(g_{j}\left(x+t h-g_{j}(x)\right) d t .\right.
\end{gathered}
$$

Agora, se $h \neq 0$, temos

$$
\begin{gathered}
\left|\sum_{j=1}^{n} h_{j} \int_{0}^{1}\left(g_{j}(x+t h)-g_{j}(x)\right) d t\right|\|h\|^{-1} \leq \\
\leq \sum_{j=1}^{n}\left|h_{j}\right|\|h\|^{-1} \max \left|g_{j}(x+t h)-g_{j}(x)\right| \leq \\
\leq \sum_{j=1}^{n} \max _{t}\left|g_{j}(x+t h)-g_{j}(x)\right| \longrightarrow 0
\end{gathered}
$$

quando $\mathrm{h} \longrightarrow 0$.

Logo $f$ é diferenciável e grad $f(x)=g(x)$. Daí concluímos que $f \in C^{1}$ e $\frac{\partial f}{\partial x_{j}}=g_{j}$. Usando indução, concluímos o teorema.

Definição 3.3 Sejam $\phi \in \mathcal{P}_{\mathrm{T}}\left(\mathbb{R}^{n}\right)$ e $m \in \mathbb{Z}^{\mathrm{n}}$. Definimos o m-ésimo coeficiente de Fourier de $\phi$ como

$$
c_{m}(\phi)=T^{-n} \int_{[0, T]^{n}} e_{-m}(x) \phi(x) d x .
$$

Teorema 3.4 Seja $\phi \in \mathcal{P}_{\mathrm{T}}\left(\mathbb{R}^{n}\right)$. Então

$$
c_{m}(\phi)=T^{-n} \int_{[0, T]^{n}} e_{-m}(x) \phi(x) d x
$$

forma uma sequência rapidamente decrescente $e$

$$
\phi=\sum_{m \in \mathbb{Z}^{n}} c_{m}(\phi) e_{m}(x)
$$


DEMONSTRAÇÃO.

$$
\begin{aligned}
T^{n} m_{j} c_{m}(\phi) & =\int_{[0, T]^{n}} m_{j} e^{-i m \omega x} \phi(x) d x=-\omega^{-1} \int_{[0, T]^{n}} D^{e_{j}} e^{-i \omega m x} \phi(x) d x= \\
& =\omega^{-1} \int_{[0, T]^{n}} e^{-i \omega m x} D^{e_{j}} \phi(x) d x=\frac{T^{n}}{\omega} c_{m} D^{e_{j}} \phi .
\end{aligned}
$$

Usando indução sobre $|\alpha|$, podemos obter $m^{\alpha} c_{m}(\phi)=\left(\omega^{-1}\right)^{|\alpha|} c_{m}\left(D^{\alpha}\right)$. Assim, para cada $\ell \in \mathbb{N}$,

$$
\begin{gathered}
|\mathrm{m}|^{\ell}\left|\mathrm{c}_{\mathrm{m}}(\phi)\right|=\sum_{|\alpha|=\ell} \frac{\ell !}{\alpha !}\left|\mathrm{m}^{\alpha} \mathrm{c}_{\mathrm{m}}(\phi)\right|=\frac{1}{\omega^{\ell} \mathrm{q}} \sum_{|\alpha|=\ell} \frac{\ell !}{\alpha !}\left|\mathrm{c}_{\mathrm{m}}\left(\mathrm{D}^{\alpha} \phi\right)\right|= \\
=\frac{1}{\omega^{\ell}} \sum_{|\alpha|=\ell} \frac{\ell !}{\alpha !}\left|\int_{[0, T]^{n}} \mathrm{D}^{\alpha} \phi(x) e^{-i \omega m x} \mathrm{~d} x\right| \leq \sum_{|\alpha|=\ell} \frac{\ell !}{\alpha !} \sup \mathrm{D}^{\alpha} \phi=M .
\end{gathered}
$$

Como $M$ não depende de $m,\left(c_{m}(\phi)\right)_{m \in \mathbb{Z}^{m}} \in s\left(\mathbb{Z}^{n} ; \mathbb{C}\right)$.

Seja agora $\psi=\sum_{m \in \mathbb{Z}^{n}} c_{m}(\phi) e_{m}$. Pelo teorema anterior, $\psi \in \mathcal{P}_{T}\left(\mathbb{R}^{n}\right)$.

$$
\begin{gathered}
c_{m}(\psi)=T^{-n} \int_{[0, T]^{n}} \psi(x) e^{-i \omega m x}=T^{-n} \int_{[0, T]^{n}} \sum_{\ell \in \mathbb{Z}^{n}} c_{\ell}(\phi) e^{i \omega(m-\ell) x} d x= \\
=T^{-n} \sum_{\ell \in \mathbb{Z}^{n}} c_{\ell} \int_{[0, T]^{n}} x e^{i \omega(m-\ell) x} d x=\frac{c_{m}(\phi)}{T^{n}} \int_{[0, T]^{n}} d x= \\
=c_{m}(\phi) .
\end{gathered}
$$

Deste modo, se provarmos que $c_{m}(\phi)=c_{m}(\psi)$ implica em $\phi=\psi$, o teorema estará demonstrado.

O próximo lema diz isso.

Lema 3.5 (Unicidade) Seja $\phi \in \mathcal{P}_{\mathrm{T}}\left(\mathbb{R}^{n}\right)$ tal que $c_{\mathrm{m}}(\phi)=0$ para todo $\mathrm{m} \in \mathbb{Z}^{\mathrm{n}}$. Então $\phi=0$.

DemonstraçÃo. Suponha que tenhamos demonstrado o lema para toda

$$
\psi: \mathbb{R}^{n} \longrightarrow \mathbb{R}, \quad \psi \in \mathcal{P}_{\mathrm{T}}\left(\mathbb{R}^{n}\right)
$$

Se $\phi \in \mathcal{P}_{\mathrm{T}}\left(\mathbb{R}^{n}\right)$, então

$$
\psi=\phi+\bar{\phi}=2 \operatorname{Re}(\phi): \mathbb{R}^{n} \longrightarrow \mathbb{R}
$$

é tal que 


$$
\mathrm{c}_{\mathrm{m}}(\psi)=\mathrm{c}_{\mathrm{m}}(\phi)+\mathrm{c}_{\mathrm{m}}(\bar{\phi})=\mathrm{c}_{\mathrm{m}}(\phi)+\mathrm{c}_{-\mathrm{m}}(\bar{\phi})=0 .
$$

Assim, $\psi=0$ e, portanto $\phi=i \xi$, onde $\xi: \mathbb{R}^{n} \longrightarrow \mathbb{R}, \xi \in \mathcal{P}_{\mathrm{T}}\left(\mathbb{R}^{n}\right)$. Mas $c_{\mathrm{m}}(\xi)=i c_{m}(\phi)=0$. Logo, $\phi=i \xi=0$.

Com isto, podemos supor, sem perda de generalidade, que $\phi$ assume somente valores reais.

Se $p(x)=\sum_{j=1}^{k} a_{j} e^{i \omega m_{j} x}$, onde $a_{j} \in \mathbb{C}$, então

$$
\begin{gathered}
\int_{B(a, T)} \phi p=\int_{B(a, T)} \phi \sum_{j=1}^{k} a_{j} e_{m_{j}}=\sum_{j=1}^{k} a_{j} \int_{B(a, T)} \phi e_{m_{j}}= \\
=T^{n} \sum_{j=1}^{k} a_{j} c_{-m_{j}}(\phi)=0 .
\end{gathered}
$$

Observe que se mostrarmos que $\phi(0)=0$ então teremos mostrado que $\phi(x)=0$, para todo $x \in \mathbb{R}^{n}$, pois

$$
\begin{gathered}
\mathrm{T}^{\mathrm{n}} \mathrm{c}_{\mathrm{m}}\left(\phi_{-x}\right)=\int_{[0, T]^{\mathrm{n}}} \phi_{-x}(\mathrm{y}) e^{-i \omega m y} \mathrm{~d} y=\int_{[0, T]^{\mathrm{n}}} \phi(x+y) e^{-i \omega m y} \mathrm{~d} y= \\
=\int_{\mathrm{B}(x, \mathrm{~T})} \phi(z) e^{-i \omega m(z-x)} \mathrm{d} z=\mathrm{T}^{\mathrm{n}} e^{i \omega m x} c_{m} \phi=0 .
\end{gathered}
$$

Logo, $\phi_{-x}(0)=0$, isto é, $\phi(x)=0$.

Mostremos enfim, que $\phi(0)=0$. Suponha que $\phi(0)=c \neq 0$.

Sem perda de generalidade, podemos supor que $c>0$, pois $c_{m}(-\phi)=-c_{m}(\phi)$ e $(-\phi)(0)=0$ se e somente se $\phi(0)=0$.

Como $\phi$ é contínua, existe $\delta>0$ tal que $0<\delta<\frac{T}{2}$ e $x \in[-\delta, \delta]^{\text {n }}$ implica em $\phi(x)>\frac{c}{2}>0$. Defina $P:[-T / 2, T / 2] \longrightarrow \mathbb{R}$ por

$$
P(t)=1+\cos (\omega t)-\cos (\omega \delta) .
$$

Se $\delta \leq|t| \leq \frac{T}{2}$ então, caso $\delta \leq t \leq \frac{T}{2}$, temos

$$
\begin{gathered}
0<\omega \delta \leq \omega t \leq \pi, \\
-1 \leq \cos \omega t \leq \cos \omega \delta<1, \\
-1-\cos \omega \delta \leq \cos \omega t-\cos \omega \delta \leq 0<1-\cos \omega \delta, \\
-1<-\cos \omega \delta \leq p(t) \leq 1<2-\cos \omega \delta .
\end{gathered}
$$

Como $p(t)=p(-t)$, segue que $|p(t)| \leq 1, \delta \leq|t| \leq \frac{T}{2}$.

Se $0 \leq|t| \leq \delta$ então $0 \leq|\omega t| \leq \omega \delta<\pi$ e $\cos \omega t=\cos |\omega t| \geq \cos \omega \delta$.

Logo, $p(t)=1+\cos \omega t-\cos \omega \delta \geq 1$.

Em seguida, para cada $a>1$ defina 


$$
p_{a}(t)=p\left(\frac{t}{a}\right)=1+\cos \frac{\omega t}{a}-\cos \omega \delta
$$

Sejam

$$
\begin{gathered}
\mathrm{q}_{\mathrm{a}}=1+\cos \frac{\omega \delta}{\mathrm{a}}-\cos \omega \delta=\min _{-\frac{\delta}{\mathrm{a}} \leq \mathrm{t} \leq \frac{\delta}{\mathrm{a}}}\{\mathrm{p}(\mathrm{t})\}, \\
\mathrm{k}=\max _{\delta \leq \mathrm{t} \leq \delta}\{\mathrm{p}(\mathrm{t})\}=2-\cos \omega \delta>1 .
\end{gathered}
$$

Como $\lim _{a \longrightarrow \infty} q_{a}=2-\cos \omega \delta=k>1$, segue que

$$
\begin{gathered}
\lim _{a \longrightarrow \infty} \frac{k}{q_{a}}=1, \\
\lim _{a \longrightarrow \infty}\left(\frac{k}{q_{a}}\right)^{2(n-1)}=1, \quad n \geq 1 .
\end{gathered}
$$

Por 3.6, existe $a_{1}$ tal que $a \geq a_{1}$ implica em

$$
\mathrm{q}_{\mathrm{a}}>\mathrm{k}-\frac{\mathrm{k}-1}{2}=\frac{\mathrm{k}+1}{2}
$$

e, por 3.7, existe $a_{2}$ tal que $a \geq a_{2}$ implica em

$$
\left(\frac{k}{q_{a}}\right)^{2(n-1)}<1+\frac{k-1}{2}=\frac{k+1}{2} .
$$

Seja $a_{0}=\max \left\{a_{1}, a_{2}\right\}$. Então para $a \geq a_{0}$, vale

$$
\left(\frac{k}{q_{a}}\right)^{2(n-1)}<\frac{k+1}{2}<q_{a} .
$$

Logo,

$$
\frac{k}{q_{a}}<\left(q_{a}\right)^{\frac{1}{2(n-1)}} \text {, isto é, } k<q_{a}^{1+\frac{1}{2(n-1}} \text {. }
$$

Deste modo, $p(t) \leq k \leq q_{a}^{1+\frac{1}{2(n-1)}}$, para todo $t \in[-\delta, \delta]$ e, se $t \in\left[-\frac{\delta}{a}, \frac{\delta}{a}\right]$, vale $\mathrm{q}_{\mathrm{a}} \leq \mathrm{p}(\mathrm{t}) \leq \mathrm{q}_{\mathrm{a}}^{1+\frac{1}{2(\mathrm{n}-1)}}$.

Fixemos $a, a \geq a_{0}$. Considere

$$
\begin{aligned}
& P(x)=\prod_{j=1}^{n} p\left(x_{j}\right)=\left(1+\cos \omega x_{1}-\cos \omega \delta\right) \cdots\left(1+\cos \omega x_{n}-\cos \omega \delta\right)= \\
& =\left(1+\frac{e^{i \omega e_{1} x}-e^{-i \omega e_{1} x}}{2}-\cos \omega \delta\right) \cdots\left(1+\frac{e^{i \omega e_{n} x}-e^{-i \omega e_{n} x}}{2}-\cos \omega \delta\right) .
\end{aligned}
$$

Para cada $N \in \mathbb{N}$, defina $P_{N}(x)=(P(x))^{N}$. $P_{N}$ é da forma $\sum_{j=1}^{k} a_{j} e^{i \omega m_{j} x}$. Temos 


$$
\int_{\left[-\frac{T}{2}, \frac{T}{2}\right]^{n}} \phi P_{N}=\int_{[-\delta, \delta]^{n}} \phi P_{N}+\int_{K} \phi P_{N}+\int_{Q} \phi P_{N},
$$

onde

$$
K=\left\{x \in[-T / 2, T / 2]^{n}:\left|x_{j}\right| \geq \delta, j=1, \ldots, n\right\}
$$

e

$$
\mathrm{Q}=\left\{x \in[-\mathrm{T} / 2, \mathrm{~T} / 2]^{\mathrm{n}}: \text { existem } i_{1}, i_{2} \text { tais que }\left|x_{i_{1}}\right| \leq \delta \text { e }\left|x_{i_{2}}\right|>\delta\right\} .
$$

No caso $\mathrm{n}=1, \mathrm{Q}=\emptyset$.

Vale que $[-\mathrm{T} / 2, \mathrm{~T} / 2]=[-\delta, \delta]^{\mathrm{n}} \cup \mathrm{K} \cup \mathrm{Q}$, união disjunta, a menos de conjuntos de medida nula.

Se $x \in[-\delta, \delta]^{n}$, então $P_{N}(x)=p^{N}\left(x_{1}\right) \ldots p^{N}\left(x_{n}\right) \geq 1^{n N}=1$, e $\phi(x) \geq c / 2$, com $\phi(0)=$ c. Logo, $\phi \mathrm{P}_{\mathrm{N}}>0 \mathrm{e}$

$$
\int_{[-\delta, \delta]^{n}} \phi P_{N} \geq \int_{\left[-\frac{\delta}{a}, \frac{\delta}{a}\right]^{n}} \phi P_{N} \geq \frac{c}{2} q_{a}^{n N}\left(\frac{2 \delta}{a}\right)^{n} .
$$

Se $x \in K$ então $\frac{T}{2} \geq\left|x_{j}\right| \geq \delta$, para todo $j=1, \ldots, n$. Assim, $\left|p\left(x_{j}\right)\right| \leq 1, j=$ $1, \ldots, n$.

Logo, $\left|\mathrm{P}_{\mathrm{N}}(x)\right| \leq 1 \mathrm{e}$

$$
\int_{K} \phi P_{N} \leq-\int_{K}\left|P_{N}\right|=-\int_{K}|\phi|\left|P_{N}\right| \leq-\sup |\phi| T^{n} .
$$

Se $x \in Q$, existem $i_{1}, i_{2} \in\{1, \ldots, n\}$, tais que $\left|x_{i_{1}}\right| \leq \delta$ e $\left|x_{i_{2}}\right|>\delta$.

Então, $\left|p\left(x_{i_{2}}\right)\right| \leq 1 \mathrm{e}$

$$
\begin{gathered}
\left|P_{N}(x)\right|=\left|p\left(x_{1}\right)\right|^{\mathfrak{n}} \ldots\left|p\left(x_{n}\right)\right|^{n} \leq \\
\leq\left|p\left(x_{1}\right)\right|^{n} \ldots\left|p\left(x_{i_{2}-1}\right)\right|^{N}\left|p\left(x_{i_{2}+1}\right)\right|^{N} \ldots\left|p\left(x_{n}\right)\right|^{N} \leq \\
\leq q^{\left(1+\frac{1}{2(n-1)}\right)(n-1) N}=q_{a}^{\left(n-\frac{1}{2}\right) N},
\end{gathered}
$$

pois se $x_{j} \in[-\delta, \delta]$, então $p\left(x_{j}\right) \leq q_{a}^{1+\frac{1}{2(n-1)}}$ e se $x_{j} \in[-T / 2, \delta] \cup[\delta, T / 2]$ então $\left|p\left(x_{j}\right)\right| \leq$ $1 \leq \mathrm{p}\left(\mathrm{x}_{\mathrm{j}}\right) \leq \mathrm{q}_{\mathrm{a}}^{1+\frac{1}{2(\mathrm{n}-1)}}$. Assim,

$$
\int_{Q} \phi P_{N} \geq-\int_{Q}|\phi|\left|P_{N}\right| \geq-\sup |\phi| q_{a}^{\left(n-\frac{1}{2}\right) N} T^{n}
$$

Portanto,

$$
\begin{gathered}
\int_{\left[-\frac{T}{2}, \frac{T}{2}\right]^{n}} \phi P_{N} \geq \frac{c}{a}\left(\frac{2 \delta}{a}\right)^{n} q_{a}^{n N}-T^{n} \sup |\phi|-T^{n} \sup |\phi| q_{a}^{\left(n-\frac{1}{2}\right) N}= \\
=q_{a}^{n N}\left(\frac{c}{2}\left(\frac{2 \delta}{a}\right)^{n}-\frac{\sup |\phi| T^{n}}{q_{a}^{n N}}-\frac{\sup |\phi| T^{n}}{q_{a}^{\frac{N}{2}}}\right) .
\end{gathered}
$$


Como $q_{a}>1$, temos

$$
\lim _{N \rightarrow \infty} q_{a}^{n N}\left(\frac{c}{2}\left(\frac{2 \delta}{a}\right)^{n}-\frac{\sup |\phi| T^{n}}{q_{a}^{n N}}-\frac{\sup |\phi| T^{n}}{q_{a}^{\frac{N}{2}}}\right)=\infty .
$$

Portanto, existe $N \in \mathbb{N}$ tal que $\int_{\left[-\frac{T}{2}, \frac{T}{2}\right]^{n}} \phi P_{N} \geq 1$. Absurdo.

Os teoremas 3.2 e 3.4 permitem escrever um isomorfismo entre $s\left(\mathbb{Z}^{n} ; \mathbb{C}\right)$ e $\mathcal{P}_{T}\left(\mathbb{R}^{n}\right)$. Defina

$$
\begin{aligned}
A: s\left(\mathbb{Z}^{\mathfrak{n}} ; \mathbb{C}\right) & \longrightarrow \mathcal{P}_{\mathrm{T}}\left(\mathbb{R}^{\mathfrak{n}}\right) \\
\left(\mathrm{c}_{\mathrm{m}}\right) & \longmapsto \sum_{\mathrm{m} \in \mathbb{Z}^{\mathrm{m}}} \mathrm{c}_{\mathrm{m}} \boldsymbol{e}_{\mathrm{m}} .
\end{aligned}
$$

A linearidade é imediata. A sobrejetividade é dada pelo teorema 3.4, visto que, para cada $\phi \in \mathcal{P}_{\mathrm{T}}\left(\mathbb{R}^{n}\right)$, a sequência $c=\left(c_{\mathrm{m}}(\phi)\right)_{\mathrm{m} \in \mathbb{Z}}$ é tal que $c \in s\left(\mathbb{Z}^{\text {n}} ; \mathbb{C}\right)$, e $A(\mathrm{c})=\phi$. Para verificarmos a injetividade, observe que se $A(c)=\sum_{m \in \mathbb{Z}^{m}} c_{m} e_{m}=0$ então para todo $\ell \in \mathbb{Z}^{n}$,

$$
\begin{aligned}
& 0=\frac{1}{T^{n}} \sum_{[0, T]^{n}}\left(\sum_{m \in \mathbb{Z}^{m}} c_{m} e_{m}\right) e_{-\ell}= \\
& =\frac{1}{T^{n}} \int_{m \in \mathbb{Z}^{n}} c_{m} \int_{[0, T]^{n}} e_{m-\ell}=c_{\ell} .
\end{aligned}
$$

\subsection{Série de Fourier em $\mathcal{P}_{T}^{\prime}\left(\mathbb{R}^{n}\right)$}

Nesta seção vemos que o conjunto das funções (distribuições) $\left\{e_{m} ; m \in \mathbb{Z}^{\mathfrak{n}}\right\}$ é uma base, no sentido de Schauder para o espaço vetorial $\mathcal{P}_{T}^{\prime}\left(\mathbb{R}^{n}\right)$. Considerando a convergência de séries em $\mathcal{P}_{T}^{\prime}\left(\mathbb{R}^{n}\right)$, todo elemento de $\mathcal{P}_{\mathrm{T}}^{\prime}\left(\mathbb{R}^{\mathfrak{n}}\right)$ é uma série trigonométrica com coeficientes de crescimento lento. Reciprocamente, toda série trigonométrica com coeficientes de crescimento lento converge em $\mathcal{P}_{T}^{\prime}\left(\mathbb{R}^{n}\right)$. Essa relação entre sequências e distribuições é um isomorfismo. O espaço das sequências de decrescimento rápido está contido no espaço das sequências de crescimento lento, o que simboliza o fato de enxergarmos uma função como distribuição.

Definição 3.8 Seja $\left(f_{m}\right)_{m \in \mathbb{Z}^{n}}$ uma sequência em $\mathcal{P}_{\mathrm{T}}^{\prime}\left(\mathbb{R}^{n}\right)$. Dizemos que a série $\sum_{m \in \mathbb{Z}^{n}} f_{m}$ é convergente em $\mathcal{P}_{T}^{\prime}\left(\mathbb{R}^{n}\right)$ se a sequência $s_{\ell}=\sum_{|m| \leq \ell} f_{m}$ for convergente em $\mathcal{P}_{\mathrm{T}}^{\prime}\left(\mathbb{R}^{n}\right)$.

Teorema 3.9 Se $f=\sum_{m \in \mathbb{Z}^{n}} f_{j} \in \mathcal{P}_{T}^{\prime}\left(\mathbb{R}^{n}\right)$ então $D^{\alpha} f=\sum_{m \in \mathbb{Z}^{n}} D^{\alpha} f_{m}$. 
DemonstraçÃo. Dados $\theta \in \mathcal{P}_{\mathrm{T}}\left(\mathbb{R}^{n}\right), \varepsilon>0$, temos que $\mathrm{D}^{\alpha} \theta \in \mathcal{P}_{\mathrm{T}}\left(\mathbb{R}^{n}\right)$ e existe $\ell_{0} \in \mathbb{N}$ tal que para todo $\ell \geq \ell_{0}$, tem-se

$$
\left|\left(f-\sum_{|m| \leq \ell} f_{m},(-1)^{\alpha} D^{\alpha} \theta\right)\right|<\varepsilon .
$$

Deste modo,

$$
\begin{gathered}
\left|\left(D^{\alpha} f-\sum_{|m| \leq \ell} D^{\alpha} f_{m}, \theta\right)\right|=\left|\left(D^{\alpha}\left(f-\sum_{|m| \leq \ell} f_{m}\right), \theta\right)\right|= \\
=\left|\left(f-\sum_{|m| \leq \ell} f_{m},(-1)^{\alpha} D^{\alpha} \theta\right)\right|<\varepsilon .
\end{gathered}
$$

Definição 3.10 Uma sequência numérica $\left(\mathrm{c}_{\mathrm{m}}\right)_{\mathfrak{m} \in \mathbb{Z}^{\mathfrak{n}}}$ é dita de crescimento lento se existirem constantes $M>0$ e $k \in \mathbb{N}$ tais que $\left|c_{m}\right| \leq M|m|^{k}$, para todo $m \in \mathbb{Z}^{m}$, $m \neq 0$.

A definição acima é equivalente a existirem constantes $M>0$ e $k \in \mathbb{N}$ tais que $\left|c_{m}\right| \leq M(1+|m|)^{k}$, para todo $m \in \mathbb{Z}^{n}$.

De fato, se $M$ e $k$ são como na definição, então

$$
\left|c_{m}\right| \leq M|m|^{k} \leq M(1+|m|)^{k} \leq M^{\prime}(1+|m|)^{k}
$$

onde $M^{\prime}=\max \left\{M,\left|c_{0}\right|\right\}$.

Agora, se existem $M>0$ e $k \in \mathbb{N}$ tais que $\left|c_{m}\right| \leq M(1+|m|)^{k}$, para todo $m \in \mathbb{Z}^{n}$, temos que, se $m \neq 0$, então $|m| \geq 1$ e

$$
\left|c_{m}\right| \leq M(1+|m|)^{k} \leq M(|m|+|m|)^{k}=2^{k} M|m|^{k} .
$$

Denotaremos por $s^{\prime}\left(\mathbb{Z}^{n} ; \mathbb{C}\right)$ o espaço das sequências de crescimento lento.

Antes de identificarmos distribuições periódicas com sequências de crescimento lento, mostremos que apesar de $\mathcal{P}_{T}^{\prime}\left(\mathbb{R}^{n}\right)$ não ser metrizável, apresenta propriedades semelhantes a de espaços métricos completos, no seguinte sentido:

Teorema 3.11 Seja $\left(f_{j}\right)_{j \in \mathbb{N}}$ uma sequência em $\mathcal{P}_{\mathrm{T}}^{\prime}\left(\mathbb{R}^{n}\right)$ satisfazendo: Dados $\theta \in$ $\mathcal{P}_{\mathrm{T}}\left(\mathbb{R}^{n}\right)$ e $\varepsilon>0$, existe $j_{0} \in \mathbb{N}$, tal que $j, k \geq j_{0}$ implica que $\left|\left(f_{j}-f_{k}, \theta\right)\right|<\varepsilon$.

Então $f_{j} \longrightarrow f$ em $\mathcal{P}_{T}^{\prime}\left(\mathbb{R}^{n}\right)$, isto é, existe $f \in \mathcal{P}_{T}^{\prime}\left(\mathbb{R}^{n}\right)$ tal que $\left(f_{j}, \theta\right) \longrightarrow(f, \theta)$, para cada $\theta \in \mathcal{P}_{\mathrm{T}}\left(\mathbb{R}^{n}\right)$.

Demonstração. Mostremos antes que se $\left(\theta_{j}\right)_{j \in \mathbb{N}}, \theta_{j} \in \mathcal{P}_{\mathrm{T}}\left(\mathbb{R}^{n}\right)$, é de Cauchy, então $\theta_{j} \longrightarrow \theta$, em $\mathcal{P}_{\mathrm{T}}\left(\mathbb{R}^{n}\right)$, para alguma $\theta \in \mathcal{P}_{\mathrm{T}}\left(\mathbb{R}^{n}\right)$, ou seja, $\mathcal{P}_{\mathrm{T}}\left(\mathbb{R}^{n}\right)$ é completo. 
Se $\left(\theta_{j}\right)_{j \in \mathbb{N}}$ é de Cauchy em $\mathcal{P}_{\mathrm{T}}\left(\mathbb{R}^{n}\right)$, então, para todo $p, k \in \mathbb{N}$ vale

$$
p_{k}\left(\theta_{j+p}-\theta_{j}\right)=\sum_{|\alpha| \leq k} \sup \left|D^{\alpha} \theta_{j+p}-D^{\alpha} \theta_{j}\right| \longrightarrow 0, \quad j \longrightarrow \infty .
$$

Também é verdade que $D^{\alpha} \theta_{j}$ converge uniformemente em $\mathbb{R}^{n}$ para uma função $\theta_{\alpha}$, T-periódica e contínua. Defina $\theta: \mathbb{R}^{n} \longmapsto \mathbb{C}$, por $\theta(x)=\lim _{j \longrightarrow \infty} \theta_{j}(x)$, considerandose a convergência pontual de $\left(\theta_{j}(x)\right)_{j \in \mathbb{N}}$.

Para cada $\psi \in \mathcal{P}_{T}\left(\mathbb{R}^{n}\right)$, usando o teorema da convergência dominada, vale

$$
\begin{gathered}
\left(\mathrm{D}^{\alpha} \theta, \psi\right)=(-1)^{|\alpha|}\left(\theta, \mathrm{D}^{\alpha} \psi\right)=(-1)^{|\alpha|} \int_{[0, T]^{\mathrm{n}}} \theta \mathrm{D}^{\alpha} \psi= \\
=\lim _{j \rightarrow \infty}(-1)^{|\alpha|} \int_{[0, \mathrm{~T}]^{n}} \theta_{j} \mathrm{D}^{\alpha} \psi=\lim _{j \rightarrow \infty} \int_{[0, T]^{n}} \mathrm{D}^{\alpha} \theta_{j} \psi=\int_{[0, T]^{\mathrm{n}}} \theta_{\alpha} \psi=\left(\theta_{\alpha}, \psi\right) .
\end{gathered}
$$

Usando o teorema 2.19, página 27, repetidas vezes, concluímos que $\theta_{\alpha} \in \mathcal{P}_{\mathrm{T}}\left(\mathbb{R}^{\mathrm{n}}\right)$ e $\mathrm{D}^{\alpha} \theta=\theta_{\alpha}$.

Concluímos então, que $\mathrm{D}^{\alpha} \theta_{j} \longrightarrow \mathrm{D}^{\alpha} \theta$ uniformemente para cada $\alpha \in \mathbb{N}^{n}$, isto é, $\theta_{j} \longrightarrow \theta$ em $\mathcal{P}_{\mathrm{T}}\left(\mathbb{R}^{\mathfrak{n}}\right)$, o que prova nossa primeira afirmação da demonstração.

Considere a sequência $\left(\rho_{j}\right)$ de funções contínuas definidas em $\mathcal{P}_{\mathrm{T}}\left(\mathbb{R}^{n}\right)$, dadas por

$$
\rho_{j}(\theta)=\left|\left(f_{j}, \theta\right)\right|, \quad j=1,2, \ldots
$$

Por uma aplicação do teorema de Baire, existe uma bola aberta em $\mathcal{P}_{\mathrm{T}}\left(\mathbb{R}^{n}\right)$, $\mathrm{B}\left(\theta_{1}, r\right)=\left\{\theta \in \mathcal{P}_{\mathrm{T}}\left(\mathbb{R}^{n}\right) ; \mathrm{d}\left(\theta, \theta_{1}\right)<r\right\}$, onde $\left(\rho_{\mathrm{j}}\right)$ é uniformemente limitada, isto é, existe $M>0$ tal que $\left|\rho_{j}(\theta)\right| \leq M$, para toda $\theta \in B\left(\theta_{1}, r\right)$ e $j \geq 1$.

Note que se $-\theta \in B\left(\theta_{1}, r\right)$, então $\rho_{j}(\theta)=\rho_{j}(-\theta) \leq M$.

Seja $\theta \in B(0, r)$. Escreva $\theta=\frac{1}{2}\left(\theta+\theta_{1}+\left(\theta-\theta_{1}\right)\right)$. Com isso,

$$
\mathrm{d}\left(\theta+\theta_{1}, \theta_{1}\right)=\sum_{\mathrm{k}=0}^{\infty} \frac{p_{\mathrm{k}}(\theta)}{2^{\mathrm{k}}\left(1+\mathrm{p}_{\mathrm{k}}(\theta)\right)}=\mathrm{d}(\theta, 0)<\mathrm{r} .
$$

Também vale que

$$
\mathrm{d}\left(-\left(\theta-\theta_{1}\right), \theta_{1}\right)=\mathrm{d}\left(\theta_{1}-\theta, \theta_{1}\right)=\sum_{\mathrm{k}=0}^{\infty} \frac{\mathrm{p}_{\mathrm{k}}(\theta)}{2^{\mathrm{k}}\left(1+\mathrm{p}_{\mathrm{k}}(\theta)\right)}=\mathrm{d}(\theta, 0)<\mathrm{r} .
$$

Sendo assim,

$$
\begin{gathered}
\rho_{j}(\theta)=\mid\left(f_{j}, \frac{1}{2}\left(\left[\theta+\theta_{1}+\left(\theta-\theta_{1}\right)\right]\right) \mid \leq \frac{1}{2}\left(\left|\left(f_{j}, \theta+\theta_{1}\right)\right|+\left|\left(f_{j}, \theta-\theta_{1}\right)\right|\right)=\right. \\
=\frac{1}{2}\left(\rho_{j}\left(\theta+\theta_{1}\right)+\rho_{j}\left(\theta-\theta_{1}\right)\right) \leq M,
\end{gathered}
$$

pois $\theta+\theta_{1},-\left(\theta-\theta_{1}\right) \in B\left(\theta_{1}, r\right)$. 
Defina $f: \mathcal{P}_{T}\left(\mathbb{R}^{n}\right) \longrightarrow \mathbb{C}$ por $f(\theta)=\lim _{j} \longrightarrow \infty\left(f_{j}, \theta\right)$. Note que $f$ está bem definida, pois para cada $\theta \in \mathcal{P}_{T}\left(\mathbb{R}^{n}\right)$, a sequência $\left(f_{j}, \theta\right)$ é de Cauchy. Mostremos que $f \in$ $\mathcal{P}_{\mathrm{T}}^{\prime}\left(\mathbb{R}^{\mathrm{n}}\right)$.

Seja $\theta_{\ell} \longrightarrow 0$ em $\mathcal{P}_{\mathrm{T}}\left(\mathbb{R}^{\mathrm{n}}\right)$.

Para cada $\varepsilon>0$, tomemos $\ell_{0} \in \mathbb{N}$ tal que $\frac{2 M \theta_{\ell}}{\varepsilon} \in B(0, r)$ sempre que $\ell \geq \ell_{0}$.

Desta forma,

$$
\rho_{j}\left(\frac{2 M \theta_{\ell}}{\varepsilon}\right)=\frac{2 M}{\varepsilon}\left|\left(f_{j}, \theta_{\ell}\right)\right| \leq M \Longrightarrow\left|\left(f_{j}, \theta_{\ell}\right)\right| \leq \frac{\varepsilon}{2},
$$

sempre que $\ell \geq \ell_{0}, j=1,2, \ldots$

Como $f\left(\theta_{\ell}\right)=\lim _{j \longrightarrow \infty}\left(f_{j}, \theta_{\ell}\right)$, para cada $\ell \geq \ell_{0}$, existe $j_{\ell}$ tal que $\left|f\left(\theta_{\ell}\right)-\left(f_{j_{\ell}}, \theta_{\ell}\right)\right|<$ $\frac{\varepsilon}{2}$.

Assim, se $\ell \geq \ell_{0}$ então

$$
\left|f\left(\theta_{\ell}\right)\right| \leq\left|f\left(\theta_{\ell}\right)-\left(f_{j_{\ell}}, \theta_{\ell}\right)\right|+\left|\left(f_{j_{\ell}}\right)\right|<\frac{\varepsilon}{2}+\frac{\varepsilon}{2}=\varepsilon .
$$

Portanto, $f \in \mathcal{P}_{\mathrm{T}}^{\prime}\left(\mathbb{R}^{n}\right)$.

Definição 3.12 Sejam $f \in \mathcal{P}_{T}^{\prime}\left(\mathbb{R}^{n}\right)$ e $m \in \mathbb{Z}^{n}$. Definimos o m-ésimo coeficiente de Fourier de f como

$$
c_{m}(f)=\frac{1}{T^{n}}\left(f, e_{-m}\right) .
$$

Teorema 3.13 Seja $\left(c_{m}\right)_{m \in \mathbb{Z}^{n}}$ uma sequência de crescimento lento. Então a série $\sum_{m \in \mathbb{Z}^{n}} c_{m} e^{i \omega m x}, \omega=2 \pi / T$ é convergente em $\mathcal{P}_{T}^{\prime}\left(\mathbb{R}^{n}\right)$. E se

$$
f=\sum_{m \in \mathbb{Z}^{n}} c_{m} e_{m}, \quad e_{m}(x)=e^{i \omega m x}
$$

então

$$
c_{m}=\frac{1}{T^{n}}\left(f, e_{-m}\right)=c_{m}(f) .
$$

DemonstraçÃo. Seja $\theta \in \mathcal{P}_{\mathrm{T}}\left(\mathbb{R}^{n}\right)$. Para cada $m=\left(m_{1}, \ldots, m_{n}\right) \in \mathbb{Z}^{n}$, temos

$$
\begin{gathered}
m_{j}\left(e_{m}, \theta\right)=\int_{[0, T]^{n}} m_{j} e^{i \omega m x} \theta(x) d x=\frac{1}{\omega} \int_{[0, T]^{n}} \frac{\partial}{\partial x_{j}}\left(e^{i \omega m x}\right) \theta(x) d x= \\
=-\frac{1}{\omega} \int_{[0, T]^{n}} e^{i \omega m x} \frac{\partial}{\partial x_{j}} \theta(x) d x .
\end{gathered}
$$

Obtemos $m_{j}\left(e_{m}, \theta\right)=\frac{1}{\omega}\left(\frac{\partial}{\partial x_{j}} e_{m}, \theta\right)=-\frac{1}{\omega}\left(e_{m}, \frac{\partial}{\partial x_{j}} \theta\right)$.

Usando indução sobre $|\alpha|$, podemos ver que

$$
\mathrm{m}^{\alpha}\left(e_{\mathrm{m}}, \theta\right)=\frac{1}{\omega^{|\alpha|}}\left(D^{\alpha} e_{\mathrm{m}}, \theta\right)=\frac{(-1)^{|\alpha|}}{\omega^{|\alpha|}}\left(e_{\mathrm{m}}, \mathrm{D}^{\alpha} \theta\right)
$$


Como $\left(c_{m}\right)_{m \in \mathbb{Z}^{n}}$ é de crescimento lento, existem $M>0$ e $k \in \mathbb{N}$ tais que $\left|c_{m}\right| \leq$ $M|m|^{k}, m \in \mathbb{Z}^{n}, m \neq 0$.

Assim,

$$
\begin{aligned}
|\mathrm{m}|^{k+n+1}\left|\left(e_{\mathrm{m}}, \theta\right)\right| & =\sum_{|\alpha|=k+n+1} \frac{k !}{\alpha !}\left|m^{\alpha}\right|\left|\left(e_{\mathrm{m}}, \theta\right)\right|=\sum_{|\alpha|=k+n+1} \frac{k !}{\alpha !} \frac{1}{\omega^{|\alpha|}}\left|\left(e_{\mathrm{m}}, \mathrm{D}^{\alpha} \theta\right)\right| \leq \\
& \leq \sum_{|\alpha|=k+n+1} \frac{k !}{\alpha !} \frac{1}{\omega^{|\alpha|}} \int_{[0, T]^{n}}\left|e^{i \omega m x} D^{\alpha} \theta(x)\right| \mathrm{d} x= \\
& =\sum_{|\alpha|=k+n+1} \frac{k !}{\alpha !} \frac{1}{\omega^{|\alpha|}} \int_{[0, T]^{n}}\left|D^{\alpha} \theta(x)\right| d x=C .
\end{aligned}
$$

Observe que $C$ não depende de $m$, e se $\theta_{j} \longrightarrow 0$ em $\mathcal{P}_{\mathrm{T}}\left(\mathbb{R}^{\mathrm{n}}\right)$, então para cada $k \in \mathbb{N},|m|^{k+n+1}\left|\left(e_{m}, \theta_{j}\right)\right| \longrightarrow 0$, e para todo $m \in \mathbb{Z}^{n}$, temos

$$
\left|c_{m}\left(e_{m}, \theta\right)\right|=\left|c_{m} \|\left(e_{m}, \theta\right)\right| \leq M|m|^{k} \frac{C}{|m|^{k+n+1}}=\frac{M C}{|m|^{n+1}}, \quad m \in \mathbb{Z}^{n}, \quad m \neq 0 .
$$

Considere a reduzida

$$
s_{j}(x)=\sum_{|m| \leq j} c_{m} e^{i \omega m x} .
$$

Para cada $p \in \mathbb{N}$, temos que

$$
\begin{gathered}
\left|\left(s_{j+p}-s_{j}, \theta\right)\right|=\left|\left(\sum_{j+1 \leq|m| \leq j+p} c_{m} e_{m}, \theta\right)\right| \leq \\
\leq \sum_{j+1 \leq|m| \leq j+p}\left|c_{m}\left(e_{m}, \theta\right)\right| \leq M C \sum_{j+1 \leq|m| \leq j+p} \frac{1}{|m|^{n+1}} .
\end{gathered}
$$

Como a série $\sum_{m \in \mathbb{Z}} \frac{1}{|m|^{n+1}}$ é convergente, é de Cauchy. Logo, $s_{j}$ também é de Cauchy e, de acordo com o teorema anterior existe $f \in \mathcal{P}_{\mathrm{T}}^{\prime}\left(\mathbb{R}^{n}\right)$ tal que

$$
f=\lim _{j \longrightarrow \infty} s_{j}=\sum_{m \in \mathbb{Z}^{n}} c_{m} e_{m}
$$

Ainda,

$$
\frac{1}{T^{n}}\left(f, e_{-m}\right)=\frac{1}{T^{n}} \sum_{\ell \in \mathbb{Z}^{n}} c_{\ell} \int_{[0, T]^{n}} e^{i \omega(\ell-m) x} d x
$$

Mas,

$$
\int_{[0, T]^{n}} e^{i \omega(\ell-m) x} d x=\left\{\begin{array}{cl}
0 & \text { se } \ell \neq m \\
T^{n} & \text { se } \ell=m
\end{array} .\right.
$$

Portanto, $c_{m}=\frac{1}{T^{n}}\left(f, e_{m}\right)$. 
O teorema acima nos diz que toda série trigonométrica tendo coeficientes de crescimento lento é convergente em $\mathcal{P}_{\mathrm{T}}^{\prime}\left(\mathbb{R}^{n}\right)$.

O teorema seguinte nos diz que toda distribuição periódica pode ser representada como uma série trigonométrica com coeficientes de crescimento lento.

Teorema 3.14 Seja $f \in \mathcal{P}_{T}^{\prime}\left(\mathbb{R}^{n}\right)$. Então $\left(c_{m}(f)\right)_{m \in \mathbb{Z}^{n}}$ é de crescimento lento e vale

$$
f=\sum_{m \in \mathbb{Z}^{n}} c_{m}(f) e_{m} .
$$

Demonstração. Como $f \in \mathcal{P}_{\mathrm{T}}^{\prime}\left(\mathbb{R}^{n}\right)$, existem $C>0$ e $k \in \mathbb{N}$ tais que

$$
\begin{gathered}
\left|c_{m}(f)\right|=\frac{1}{T^{n}}\left|\left(f, e_{-m}\right)\right| \leq C \sum_{|\alpha| \leq k} \sup _{x \in \mathbb{R}^{n}}\left|D^{\alpha} e_{m}(x)\right|= \\
=\frac{C}{T^{n}} \sum_{|\alpha| \leq k} \sup _{x \in \mathbb{R}^{n}}\left|\omega^{|\alpha|} m^{\alpha} e_{m}(x)\right|=\frac{C}{T^{n}} \sum_{|\alpha| \leq k} \sup _{x \in \mathbb{R}^{n}}\left|\omega^{|\alpha|} m^{\alpha}\right| \leq \\
\leq \frac{C \max \left\{1, \omega^{k}\right\}}{T^{n}} \sum_{|\alpha| \leq k}\left|m^{\alpha}\right| \leq C^{\prime} \sum_{|\alpha| \leq k}(1+|m|)^{|\alpha|} \leq C^{\prime \prime}(1+|m|)^{k},
\end{gathered}
$$

com $C^{\prime}>0, C^{\prime \prime}>0$ convenientes, para todo $m \in \mathbb{Z}^{n}$.

Portanto $\left(c_{m}(f)\right)_{m \in \mathbb{Z}^{n}}$ é de crescimento lento. Pelo teorema anterior, existe $g \in$ $\mathcal{P}_{T}^{\prime}\left(\mathbb{R}^{n}\right)$ tal que

$$
g=\sum_{m \in \mathbb{Z}^{n}} c_{m}(f) e_{m}
$$

Resta mostrar que $\mathrm{g}=\mathrm{f}$. Para isto, mostremos primeiramente que $\delta=\mathrm{T}^{-\mathrm{n}} \sum_{\mathrm{m} \in \mathbb{Z}^{n}} e_{\mathrm{m}}$.

Para cada $\theta \in \mathcal{P}_{\mathrm{T}}\left(\mathbb{R}^{n}\right)$, temos

$$
\begin{gathered}
\left(\frac{1}{T^{n}} \sum_{|m| \leq j} e_{m}-\delta, \theta\right)=\frac{1}{T^{n}} \sum_{|m| \leq j} \int_{[0, T]^{n}} e_{m}(x) \theta(x) d x-\theta(0) \\
=\frac{1}{T^{n}} \sum_{|m| \leq j} \int_{[0, T]^{n}} e_{m}(x) \theta(x) d x-\frac{\theta(0)}{T^{n}} \int_{[0, T]^{n}} e_{m}(x) d x= \\
=\frac{1}{T^{n}} \sum_{|m| \leq j} \int_{[0, T]^{n}} e_{m}(x)(\theta(x)-\theta(0)) d x= \\
=\sum_{|m| \leq j} c_{m}(\theta-\theta(0))=\sum_{|m| \leq j} c_{m}(\theta-\theta(0)) e_{m}(0) \longrightarrow \\
\longrightarrow(\theta-\theta(0))(0)=\theta(0)-\theta(0)=0 .
\end{gathered}
$$


Usando a continuidade sequencial em cada variável da T-convolução, chegamos a

$$
\begin{gathered}
(f, \theta)=(f * \delta, \theta)=\left(\frac{1}{T^{n}} \sum_{m \in \mathbb{Z}^{n}} f * e_{m}, \theta\right)=\left(\frac{1}{T^{n}} \sum_{m \in \mathbb{Z}^{n}}\left(f,\left(\check{e_{m}}\right)_{a}\right), \theta(a)\right)= \\
=\left(\frac{1}{T^{n}} \sum_{m \in \mathbb{Z}^{n}}\left(f, e_{-m}\right) e_{m}(a), \theta(a)\right)\left(\sum_{m \in \mathbb{Z}^{n}} c_{m}(f) e_{m}(a), \theta(a)\right)=(g, \theta) .
\end{gathered}
$$

Portanto, $f=g$.

Estes dois últimos teoremas mostram que é possível estabelecer um isomorfismo entre $s^{\prime}\left(\mathbb{Z}^{n} ; \mathbb{C}\right)$ e $\mathcal{P}_{\mathrm{T}}^{\prime}\left(\mathbb{R}^{n}\right)$.

Defina

$$
\begin{aligned}
B: s^{\prime}\left(\mathbb{Z}^{n} ; \mathbb{C}\right) & \longrightarrow \mathcal{P}_{T}^{\prime}\left(\mathbb{R}^{n}\right) \\
& c=\left(c_{m}\right) \longmapsto \sum_{m \in \mathbb{Z}^{n}} c_{m} e_{m}
\end{aligned}
$$

A linearidade de B é trivial.

A sobrejetividade decorre do último teorema, pois para cada $f \in \mathcal{P}_{T}^{\prime}\left(\mathbb{R}^{n}\right)$, tome $c=\left(c_{m}(f)\right)$ e então teremos $B(c)=f$.

Para verificar a injetividade, note que se $B(c)=\sum_{m \in \mathbb{Z}^{n}} c_{m} e_{m}=0$, então o Teorema 3.13 diz que $c_{m}=\left(0, e_{-m}\right)=0$, isto é, $c=0$.

Exemplo 3.15 Já sabemos que $\delta=\frac{1}{T^{n}} \sum_{m \in \mathbb{Z}^{n}} e_{m}$. Calculemos a série de $\mathrm{D}^{\alpha} \delta$, $\alpha \in \mathbb{N}$.

$$
\begin{gathered}
c_{m}\left(D^{\alpha} \delta\right)=\frac{1}{T^{n}}\left(D^{\alpha} \delta, e_{-m}\right)=\frac{(-1)^{|\alpha|}}{T^{n}}\left(\delta, D^{\alpha} e_{-m}\right)= \\
=\frac{(-1)^{|\alpha|}}{T^{n}}\left(\delta,(-1)^{|\alpha|} \omega^{|\alpha|} m^{\alpha} e_{-m}\right)=\frac{\omega^{|\alpha|}}{T^{n}} m^{\alpha} .
\end{gathered}
$$

$\log 0$

$$
\mathrm{D}^{\alpha} \delta=\frac{1}{\mathrm{~T}^{n}} \sum_{\mathrm{m} \in \mathbb{Z}^{n}} \omega^{|\alpha|} \mathrm{m}^{\alpha} e_{\mathrm{m}} .
$$

Teorema 3.16 Se f, $g \in \mathcal{P}_{\top}^{\prime}\left(\mathbb{R}^{n}\right)$ então

$$
f * g=T^{n} \sum_{m \in \mathbb{Z}^{n}} c_{m}(f) c_{m}(g) e_{m}
$$


DEMONSTRAÇÃO.

$$
\begin{gathered}
c_{m}(f * g)=\frac{1}{T^{n}}\left(f * g, e_{-m}\right)=\frac{1}{T^{n}}\left(f * g * e_{m}\right)(0)= \\
=\frac{1}{T^{n}}\left(f *\left(g * e_{m}\right)\right)(0)=\frac{1}{T^{n}}\left(f(x),\left(g * e_{m}\right)^{-}(x)\right)= \\
=\frac{1}{T^{n}}\left(f(x),\left(g * e_{m}\right)(-x)\right)=\frac{1}{T^{n}}\left(f(x),\left(g(a), e_{m}(-x-a)\right)=\right. \\
=\frac{1}{T^{n}}\left(f(x),\left(g(a), e_{-m}(a)\right) e_{-m}(x)\right)=\frac{1}{T^{n}}\left(f(x), e_{-m}(x)\right)\left(g(a), e_{-m}(a)\right)= \\
=T^{n} c_{m}(f) c_{m}(g) .
\end{gathered}
$$

Logo,

$$
\mathrm{f} * \mathrm{~g}=\mathrm{T}^{\mathrm{n}} \sum_{\mathrm{m} \in \mathbb{Z}^{\mathfrak{n}}} \mathrm{c}_{\mathrm{m}}(\mathrm{f}) \mathrm{c}_{\mathrm{m}}(\mathrm{g}) \boldsymbol{e}_{\mathrm{m}}
$$

\subsection{Série Parcial de Fourier em $\mathcal{P}_{T}\left(\mathbb{R}^{n}\right)$}

Sejam $p, q, n \in \mathbb{N}$, tais que $n=p+q$. Considere a soma direta $\mathbb{R}^{n}=\mathbb{R}^{p} \oplus \mathbb{R}^{q}$. Escrevemos $(x, y) \in \mathbb{R}^{n}$ para indicar que $x \in \mathbb{R}^{p}$ e $y \in \mathbb{R}^{q}$. Dada $\phi \in \mathcal{P}_{\mathrm{T}}\left(\mathbb{R}^{n}\right)$, mostraremos que existe uma maneira natural de se considerar a série de Fourier de $\phi$ com relação a apenas uma destas variáveis. Mais precisamente, dada $\phi \in \mathcal{P}_{\mathrm{T}}\left(\mathbb{R}^{n}\right)$, considere para cada $x \in \mathbb{R}^{p}$, a função

$$
\begin{aligned}
\psi^{x}: \mathbb{R}^{q} & \longrightarrow \mathbb{C} \\
y & \longmapsto \phi(x, y)
\end{aligned} .
$$

Note que $\psi^{x} \in \mathcal{P}_{\mathrm{T}}\left(\mathbb{R}^{\mathrm{q}}\right)$ e, portanto,

$$
\phi(x, y)=\psi^{x}(y)=\sum_{m \in \mathbb{Z}^{q}} c_{m}\left(\psi^{x}\right) e^{i \omega m y},
$$

onde

$$
c_{m}\left(\psi^{x}\right)=\frac{1}{T^{q}} \int_{[0, T] q} \psi^{x}(y) e^{-i \omega m y} d y=\frac{1}{T^{q}} \int_{[0, T] q} \phi(x, y) e^{-i \omega m y} d y .
$$

Definição 3.17 Seja $\phi_{\mathrm{m}}: \mathbb{R}^{p} \longrightarrow \mathbb{C}$ dada por

$$
\phi_{\mathfrak{m}}(x)=c_{m}\left(\psi^{x}\right)=\frac{1}{\mathrm{~T}^{q}} \int_{[0, T] q} \phi(x, y) e^{i \omega m y} .
$$

Dizemos que $\phi_{\mathrm{m}}(\mathrm{x})$ é o $\mathrm{m}$-ésimo coeficiente parcial de Fourier, $\mathrm{m} \in \mathbb{Z}^{\mathrm{q}}$, da série parcial de Fourier

$$
\sum_{m \in \mathbb{Z} q} \phi_{\mathfrak{m}}(x) e^{-i m y}
$$


Toda $\phi \in \mathcal{P}_{\mathrm{T}}\left(\mathbb{R}^{n}\right)$ possui série de parcial de Fourier convergindo para a mesma. Seus coeficientes são caracterizados por funções em $\mathcal{P}_{\mathrm{T}}\left(\mathbb{R}^{p}\right)$ que decaem uniformemente como sequências de decrescimento rápido. Mostrar isso é o objetivo desta seção.

Teorema 3.18 Se $\phi_{\mathrm{m}}$ é como acima então $\phi_{\mathrm{m}} \in \mathcal{P}_{\mathrm{T}}\left(\mathbb{R}^{\mathrm{p}}\right), \mathrm{m} \in \mathbb{Z}^{\mathrm{q}}$.

Demonstração. Temos que $\phi(x, y)=\sum_{m \in \mathbb{Z}^{q}} \phi_{m}(x) e^{i \omega m y}$ e $\phi_{m}$ é T-periódica.

Mostremos que $\phi_{m}$ é contínua. De fato, se $x_{j} \longrightarrow x_{0}$ em $\mathbb{R}^{p}$ então

$$
\lim _{j \longrightarrow \infty} \phi\left(x_{j}, y\right) e^{-i \omega m y}=\phi\left(x_{0}, y\right) e^{-i \omega m y}
$$

e como $\phi\left(x_{j}, y\right) e^{-i \omega m y}$ é mensurável e $\left|\phi\left(x_{j}, y\right) e^{-i \omega m y}\right|=\left|\phi\left(x_{j}, y\right)\right| \leq C$ (onde $C$ não depende de $j$, pois $\phi$ é limitada), pelo teorema da Convergência Dominada, temos que

$$
\begin{gathered}
\lim _{j \longrightarrow \infty} \phi_{m}\left(x_{j}\right)=\frac{1}{\mathrm{Tq}^{q}} \lim _{j \longrightarrow \infty} \int_{[0, \mathrm{~T}]^{q}} \phi\left(x_{j}, y\right) e^{-i \omega m y} d y= \\
=\frac{1}{\mathrm{~T}^{q}} \int_{[0, T] q} \phi\left(x_{0}, y\right) e^{-i \omega m y} \mathrm{~d} y=\phi_{\mathfrak{m}}\left(x_{0}\right) .
\end{gathered}
$$

Para verificarmos que $\phi_{m}$ é $C^{1}$, considere uma sequência $\left(h_{j}\right), h_{j} \in \mathbb{R}^{*}, h_{j} \longrightarrow 0$. Temos que

$$
\lim _{j \longrightarrow \infty} \frac{\phi\left(x+h_{j} e_{k}, y\right)-\phi(x, y)}{h_{j}} e^{-i \omega m y}=\frac{\partial}{\partial x_{k}} \phi(x, y) e^{-i \omega m y}
$$

Além disto,

$$
\left|\frac{\partial \phi(x, y)}{\partial x_{k}} e^{-i \omega m y}\right|=\left|\frac{\partial \phi(x, y)}{\partial x_{k}}\right| \leq C, \quad(x, y) \in \mathbb{R}^{n} .
$$

Deste modo, a sequência

$$
\frac{\phi\left(x+h_{j} e_{k}, y\right)-\phi(x, y)}{h_{j}} e^{-i \omega m y}
$$

também é limitada para todo $(x, y) \in \mathbb{R}^{n}$.

Pelo teorema da Convergência Dominada, segue que

$$
\begin{gathered}
\lim _{j \longrightarrow \infty} \frac{\phi_{m}\left(x+h_{j} e_{k}\right)-\phi_{m}(x)}{h_{j}}= \\
=\frac{1}{\mathrm{~T}^{\mathrm{q}}} \lim _{j \longrightarrow \infty} \int_{[0, \mathrm{~T}]^{q}} \frac{\phi_{m}\left(x+h_{j} e_{k}, y\right)-\phi(x, y)}{h_{j}} e^{-i \omega m y} d y= \\
=\frac{1}{\mathrm{~T}^{q}} \int_{[0, T]^{q}} \frac{\partial}{\partial x_{k}} \phi(x, y) e^{-i \omega m y} d y .
\end{gathered}
$$


Isto é,

$$
\frac{\partial}{\partial x_{k}} \phi_{m}(x)=\frac{1}{T^{q}} \int_{[0, T] q} \frac{\partial}{\partial x_{k}} \phi(x, y) e^{-i \omega m y} d y .
$$

Como $\frac{\partial}{\partial x_{k}} \phi(x, y)$ é contínua, podemos usar o mesmo raciocínio feito na prova de que $\phi_{m}$ é contínua, para concluirmos que $\frac{\partial}{\partial x_{k}} \phi_{m}(x)$ também é.

Por indução na ordem da derivada, concluímos que $\phi_{\mathrm{m}} \in \mathcal{P}_{\mathrm{T}}\left(\mathbb{R}^{p}\right)$.

Teorema 3.19 Seja $\left(\phi_{\mathrm{m}}\right)_{\mathfrak{m} \in \mathbb{Z}^{q}}$ uma sequência de funções em $\mathcal{P}_{\mathrm{T}}\left(\mathbb{R}^{p}\right)$ tal que para cada $\alpha \in \mathbb{N}^{p}$, e $N \in \mathbb{N}$, existe $C>0$, tal que

$$
\left|\mathrm{D}^{\alpha} \phi_{\mathrm{m}}(x)\right| \leq \frac{\mathrm{C}}{(1+|\mathrm{m}|)^{\mathrm{N}}}
$$

para todo $\mathrm{m} \in \mathbb{Z}^{\mathrm{q}}$, e $x \in \mathbb{R}^{\mathrm{p}}$. Então, a função $\psi: \mathbb{R}^{\mathrm{p}+\mathrm{q}} \longrightarrow \mathbb{C}$ dada por

$$
\psi(x, y)=\sum_{m \in \mathbb{Z} q} \phi_{m}(x) e^{i \omega m y}
$$

está bem definida e pertence a $\mathcal{P}_{\mathrm{T}}\left(\mathbb{R}^{\mathfrak{n}}\right)$.

Demonstração. Como para cada $\chi \in \mathbb{R}^{p}, \sum_{m \in \mathbb{Z}^{q}} \phi_{m}(\chi) e^{i \omega m y}$ é convergente, $\psi$ está bem definida. Seja

$$
\begin{aligned}
\psi^{x}: \mathbb{R}^{q} & \longrightarrow \mathbb{C} \\
y & \longmapsto \psi(x, y)
\end{aligned} .
$$

Note que $\psi^{x}$ é periódica e $\psi^{x} \in \mathcal{P}_{\mathrm{T}}\left(\mathbb{R}^{q}\right)$, pois dado $N \in \mathbb{N}$, existe $C>0$ tal que

$$
\left|c_{m}\left(\psi^{x}\right)\right|=\left|\phi_{m}(x)\right| \leq \frac{C}{(1+|m|)^{N}} .
$$

Mostremos que $\psi$ é contínua. Seja $\left(x_{0}, y_{0}\right) \in \mathbb{R}^{n}$.

$$
\begin{gathered}
\left.\left|\psi(x, y)-\psi\left(x_{0}, y_{0}\right)\right|=\mid \sum_{m \in \mathbb{Z}^{q}}\left(\phi_{\mathfrak{m}}(x) e^{i \omega m y}\right)-\phi_{\mathfrak{m}}\left(x_{0}\right) e^{i \omega m y_{0}}\right) \mid= \\
\left|\sum_{\mathfrak{m} \in \mathbb{Z}^{q}}\left(\phi_{\mathfrak{m}}(x)-\phi_{\mathfrak{m}}\left(x_{0}\right)\right) e^{i \omega m y}+\phi_{\mathfrak{m}}\left(x_{0}\right)\left(e^{i \omega m y}-e^{i \omega m y_{0}}\right)\right| \leq \\
\leq \sum_{\mathfrak{m} \in \mathbb{Z}^{q}}\left|\phi_{m}(x)-\phi_{\mathfrak{m}}\left(x_{0}\right)\right|+\left|\sum_{\mathfrak{m} \in \mathbb{Z}^{q}} \phi_{\mathfrak{m}}\left(x_{0}\right)\left(e^{i \omega m y}-e^{i \omega m y_{0}}\right)\right|= \\
=\sum_{m \in \mathbb{Z}^{q}}\left|\phi_{\mathfrak{m}}(x)-\phi_{\mathfrak{m}}\left(x_{0}\right)\right|+\left|\psi^{x_{0}}(y)-\psi^{x_{0}}\left(y_{0}\right)\right| .
\end{gathered}
$$

Como existe $\mathrm{C}_{\mathrm{q}+1}>0$ tal que

$$
\left|\phi_{m}(x)-\phi_{m}\left(x_{0}\right)\right| \leq \frac{C_{q+1}}{(1+|m|)^{q+1}}, \quad m \in \mathbb{Z}^{q}
$$


a função $\sum_{m \in \mathbb{Z} q}\left|\phi_{m}(x)-\phi_{m}\left(x_{0}\right)\right|$ é contínua. Como $\psi^{x_{0}}$ também o é, dado $\varepsilon>0$, existe $\delta>0$ tal que $|(x, y)|<\delta$ implica que

$$
\left|\psi(x, y)-\psi\left(x_{0}, y_{0}\right)\right| \leq \sum_{m \in \mathbb{Z}^{q}}\left|\phi_{m}(x)-\phi\left(x_{0}\right)\right|+\left|\psi^{x_{0}}(y)-\psi^{x_{0}}\left(y_{0}\right)\right|<\frac{\varepsilon}{2}+\frac{\varepsilon}{2}=\varepsilon .
$$

Vejamos agora que $\psi \in \mathbb{C}^{1}$

$$
\begin{gathered}
\left|\frac{\psi\left(x_{0}+h e_{j}, y_{0}\right)-\psi\left(x_{0}, y_{0}\right)}{h}-\sum_{m \in \mathbb{Z} q} \frac{\partial}{\partial x_{j}} \phi_{m}\left(x_{0}\right) i m y_{0}\right|= \\
=\mid \frac{\sum_{m \in \mathbb{Z}^{q}} \phi_{m}\left(x_{0}+h e_{j}\right) e^{i \omega m y_{0}}-\sum_{m \in \mathbb{Z}^{q}} \phi_{m}\left(x_{0}\right) e^{i \omega m y_{0}}}{h}- \\
-\sum_{m \in \mathbb{Z} q} \frac{\partial}{\partial x_{j}} \phi_{m}\left(x_{0}\right) e^{i \omega m y_{0}} \mid= \\
=\left|\sum_{m \in \mathbb{Z}^{q}}\left(\frac{\phi_{m}\left(x_{0}+h e_{j}\right)-\phi_{m}\left(x_{0}\right)}{h}-\frac{\partial}{\partial x_{j}} \phi_{m}\left(x_{0}\right)\right) e^{i \omega m y_{0}}\right| \leq \\
\leq \sum_{m \in \mathbb{Z}^{q}}\left|\frac{\phi_{\mathfrak{m}}\left(x_{0}+h e_{j}\right)-\phi_{m}\left(x_{0}\right)}{h}-\frac{\partial}{\partial x_{j}} \phi_{m}\left(x_{0}\right)\right|= \\
=\sum_{m \in \mathbb{Z}^{q}}\left|\frac{\partial \phi_{m}}{\partial x_{j}}\left(x_{0}+\xi e_{j}\right)-\frac{\partial \phi_{m}}{\partial x_{j}}\left(x_{0}\right)\right|= \\
=\sum_{m \in \mathbb{Z}^{q}}\left|\frac{\partial^{2}}{\partial x_{j}^{2}} \phi_{m}\left(x_{0}+\zeta_{m} e_{j}\right)\right| \xi_{m} \mid,
\end{gathered}
$$

onde

$$
\left|\zeta_{m}\right|<\left|\xi_{m}\right|<|h|
$$

Existe $C_{q}>0$, tal que

$$
\left|\frac{\partial^{2}}{\partial x_{j}^{2}} \phi_{m}\left(x_{0}+\zeta_{m} e_{j}\right)\right| \leq \frac{C_{q}}{(1+|m|)^{q+1}},
$$

$\mathrm{m} \in \mathbb{Z}^{\mathrm{q}}$. Sendo assim, dado $\varepsilon>0$, tome

$$
\delta=\left(C_{\mathrm{q}} \sum_{\mathrm{m} \in \mathbb{Z} q} \frac{1}{(1+|\mathrm{m}|)^{\mathrm{q}+1}}\right)^{-1} \mathcal{\varepsilon},
$$

e, se $|h| \leq \delta$ então $\left|\xi_{m}\right|<\delta$ e, portanto,

$$
\begin{gathered}
\sum_{m \in \mathbb{Z}^{q}}\left|\frac{\partial^{2}}{\partial x_{j}^{2}} \phi_{m}\left(x_{0}+\zeta_{m} e_{j}\right)\right|\left|\xi_{m}\right|<\sum_{m \in \mathbb{Z}^{q}}\left|\frac{\partial^{2}}{\partial x_{j}^{2}} \phi_{m}\left(x_{0}+\zeta_{m} e_{j}\right)\right| \delta \leq \\
\leq C_{q} \delta \sum_{m \in \mathbb{Z}^{q}} \frac{1}{(1+|m|)^{q+1}}=\varepsilon .
\end{gathered}
$$


Ou seja,

$$
\frac{\partial \psi}{\partial x_{j}}\left(x_{0}, y_{0}\right)=\sum_{m \in \mathbb{Z}^{q}} \frac{\partial}{\partial x_{j}} \phi_{m}\left(x_{0}\right) e^{i \omega m y_{0}}
$$

Note que

$$
\left|\frac{\partial}{\partial x_{j}} \phi_{m}(x) e^{i \omega m y}\right|=\left|\frac{\partial}{\partial x_{j}} \phi_{m}(x)\right| \leq \frac{C_{q+1}}{(1+|m|)^{q+1}},
$$

para todo $m \in \mathbb{Z}^{q}$. Segue então que $\frac{\partial \psi}{\partial x_{j}}$ é contínua, já que

$$
\begin{gathered}
\lim _{h \longrightarrow 0} \frac{\psi\left(x_{0}, y_{0}+h e_{j}\right)-\psi\left(x_{0}, y_{0}\right)}{h}=\lim _{h \longrightarrow 0} \frac{\psi^{x_{0}}\left(y_{0}+h e_{j}\right)-\psi^{x_{0}}\left(y_{0}\right)}{h}= \\
=\frac{\partial}{\partial y_{j}} \psi^{x_{0}}\left(y_{0}\right)=\left.\frac{\partial}{\partial y_{j}}\left(\sum_{m \in \mathbb{Z} q} \phi_{m}\left(x_{0}\right) e^{i \omega m y}\right)\right|_{y=y_{0}}=i \omega \sum_{m \in \mathbb{Z} q} m_{j} \phi_{m}\left(x_{0}\right) e^{i \omega m y_{0}},
\end{gathered}
$$

e

$$
\left|m_{j} \phi_{m}\left(x_{0}\right) e^{i \omega m y_{0}}\right|=\left|m_{j} \phi_{m}\left(x_{0}\right)\right| \leq \frac{E}{(1+|m|)^{q+1}}, \quad m \in \mathbb{Z}^{q},
$$

para algum $E>0$ conveniente.

Mostremos por indução sobre a ordem das derivadas, que $\psi \in \mathbb{C}^{\infty}$. Suponha que $\partial^{(\alpha, \beta)}$ é contínua, e que

$$
\partial^{(\alpha, \beta)} \psi(x, y)=\sum_{m \in \mathbb{Z} q} \partial^{\alpha} \phi_{m}(x)(i \omega m)^{\beta} e^{i \omega m y} .
$$

Defina $\alpha_{j}=\alpha+e_{j}, \alpha_{j j}=\alpha_{j}+e_{j}$.

$$
\begin{aligned}
& |h|^{-1} \mid \partial^{(\alpha, \beta)} \psi\left(x_{0}+h e_{j}, y_{0}\right)-\partial^{(\alpha, \beta)} \psi\left(x_{0}, y_{0}\right)- \\
& -|h| \sum_{m \in \mathbb{Z}^{q}} \partial^{\alpha_{j}} \phi_{m}\left(x_{0}\right)(i \omega m)^{\beta} e^{i \omega m y_{0}} \mid= \\
& =\left|\sum_{\mathfrak{m} \in \mathbb{Z}^{q}}\left(|h|^{-1}\left(\partial^{\alpha} \phi_{\mathfrak{m}}\left(x_{0}+h e_{j}\right)-\partial^{\alpha} \phi_{\mathfrak{m}}\left(x_{0}\right)\right)-\partial^{\alpha_{j}} \phi_{\mathfrak{m}}\left(x_{0}\right)\right)(i \omega m)^{\beta} e^{i \omega m y_{0}}\right| \leq \\
& \leq\left.\sum_{\mathfrak{m} \in \mathbb{Z} q}|| h\right|^{-1}\left(\partial^{\alpha} \phi_{\mathfrak{m}}\left(x_{0}+h e_{j}\right)-\partial^{\alpha} \phi_{\mathfrak{m}}\left(x_{0}\right)\right)-\partial^{\alpha_{j}} \phi_{\mathfrak{m}}\left(x_{0}\right)||(i \omega m)^{\beta} \mid= \\
& =\sum_{m \in \mathbb{Z}^{q}}\left|\left(\partial^{\alpha_{j}} \phi_{m}\left(x_{0}+\xi_{m} e_{j}\right)-\partial^{\alpha_{j}} \phi_{m}\left(x_{0}\right)\right) \|(i \omega m)^{\beta}\right|= \\
& =\sum_{m \in \mathbb{Z}^{q}}\left|\partial^{\alpha_{j j}} \phi_{m}\left(x_{0}+\zeta_{m} e_{j}\right)\left\|\xi_{m}\right\|(i \omega m)^{\beta}\right|,
\end{aligned}
$$


onde $\left|\zeta_{m}\right|<\left|\xi_{m}\right|<|h|$. Como existe D $>0$ tal que

$$
\left|\partial^{\alpha_{j} j} \phi_{m}\left(x_{0}+\xi_{m} e_{j}\right) \|(i \omega m)^{\beta}\right| \leq \frac{D}{(1+|m|)^{q+1}},
$$

para todo $m \in \mathbb{Z}^{\mathrm{q}}$, tome, para cada $\varepsilon>0$,

$$
\delta=\varepsilon\left(D \sum_{m \in \mathbb{Z}^{q}} \frac{1}{(1+|m|)^{q+1}}\right)^{-1} .
$$

Então, se $h$ é tal que $|h|<\delta$, temos

$$
\sum_{m \in \mathbb{Z}^{q}}\left|\partial^{\alpha_{j} j} \phi_{m}\left(x_{0}+\zeta e_{j}\right)\left\|\xi_{m}\right\|(i \omega m)^{\beta}\right|<\varepsilon .
$$

Desta forma,

$$
\partial^{\left(\alpha_{j}, \beta\right)} \psi(x, y)=\sum_{m \in \mathbb{Z}^{q}} \partial^{\alpha_{j}} \phi_{m} e^{i \omega m y}(i \omega m)^{\beta}
$$

Como

$$
\left|\partial^{\alpha_{j}} \phi_{m}(x) e^{i \omega m y}(i \omega m)^{\beta}\right|=\left|\partial^{\alpha_{j}} \phi_{m}(x)(i \omega m)^{\beta}\right| \leq \frac{D}{(1+|m|)^{q+1}}, \quad m \in \mathbb{Z}^{q},
$$

segue que $\partial^{\left(\alpha_{j}, \beta\right)} \psi$ é contínua. Defina $\beta_{j}=\beta+e_{j}$,

$$
\begin{gathered}
\lim _{h \longrightarrow 0} h^{-1}\left[\partial^{(\alpha, \beta)} \psi\left(x_{0}, y_{0}+h e_{j}\right)-\partial^{(\alpha, \beta)} \psi\left(x_{0}, y_{0}\right)\right]= \\
=\lim _{h \longrightarrow 0} h^{-1} \sum_{m \in \mathbb{Z} q} \partial^{\alpha} \phi_{m}\left(x_{0}\right)(i \omega m)^{\beta} e^{i \omega m y_{0}}\left(e^{i \omega h m e_{j}}-1\right)= \\
=\sum_{m \in \mathbb{Z}^{q}} \partial^{\alpha} \phi_{m}\left(x_{0}\right)(i \omega m)^{\beta}\left(i \omega m_{j}\right) e^{i \omega m y_{0}}= \\
=\sum_{m \in \mathbb{Z} q} \partial^{\alpha} \phi_{m}\left(x_{0}\right)(i \omega m)^{\beta_{j}} e^{i \omega m y_{0}} .
\end{gathered}
$$

Deste modo, $\partial^{\left(\alpha, \beta_{j}\right)} \psi$ existe e é contínua. A periodicidade de $\psi$ é imediata.

Teorema 3.20 Se $\phi \in \mathcal{P}_{\mathrm{T}}\left(\mathbb{R}^{n}\right)$ então

$$
\phi(x, y)=\sum_{m \in \mathbb{Z} q} \phi_{m}(x) e^{i \omega m y}
$$

onde $\phi_{\mathrm{m}} \in \mathcal{P}_{\mathrm{T}}\left(\mathbb{R}^{\mathrm{p}}\right) e$

$$
\phi_{\mathrm{m}}(x)=\frac{1}{\mathrm{~T}^{\mathrm{q}}} \int_{[0, \mathrm{~T}]^{q}} \phi(x, y) e^{-i \omega m y} \mathrm{~d} y .
$$

Além disto, dados $\mathrm{k} \in \mathbb{N}$ e $\alpha \in \mathbb{N}^{p}$, existe $\mathrm{C}>0$ tal que

$$
\left|D^{\alpha} \phi_{m}(x)\right| \leq \frac{C}{(1+|m|)^{k}}
$$

para todo $m \in \mathbb{Z}^{q}$ e $x \in \mathbb{R}^{p}$. 
DEMONSTRAÇÃo. Só resta demonstrar a última parte do teorema. Sejam $\alpha \in \mathbb{N}^{p}$ e $\beta \in \mathbb{N}^{q}$.

$$
\begin{gathered}
\mathrm{T}^{\mathrm{q}}\left|\mathrm{m}^{\beta} \mathrm{D}^{\alpha} \phi_{\mathrm{m}}(x)\right|=\left|\int_{[0, \mathrm{~T}]^{q}} \mathrm{D}^{(\alpha, 0)} \phi(x, y) \mathrm{m}^{\beta} e^{-i m y} \mathrm{dy}\right|= \\
=\frac{1}{\omega^{|\beta|} \mid}\left|\int_{[0, \mathrm{~T}] \mathrm{q}} \mathrm{D}^{(\alpha, 0)} \phi(x, y) \mathrm{D}^{(0, \beta)} e^{-i \omega m y} \mathrm{dy}\right|= \\
=\frac{1}{\omega^{|\beta|} \mid}\left|\int_{[0, \mathrm{~T}] \mathrm{q}} \mathrm{D}^{(\alpha, \beta)} \phi(x, y) e^{-i \omega m y} \mathrm{dy}\right| .
\end{gathered}
$$

Dado $k \in \mathbb{N}$, temos

$$
\begin{gathered}
|\mathrm{m}|^{k}\left|\mathrm{D}^{\alpha} \phi_{\mathfrak{m}}(x)\right|=\sum_{|\beta|=k} \frac{k !}{\beta !}\left|m^{\beta}\right|\left|\mathrm{D}^{\alpha} \phi_{\mathfrak{m}}(x)\right|=\sum_{|\beta|=k}\left|\frac{k !}{\beta !} m^{\beta} \mathrm{D}^{\alpha} \phi_{\mathfrak{m}}(x)\right|= \\
=\sum_{|\beta|=k} \frac{k !}{\beta !} \frac{1}{\omega^{|\beta|} \mid}\left|\frac{1}{\mathrm{Tq}} \int_{[0, \mathrm{~T}] \mathrm{q}} \mathrm{D}^{(\alpha, \beta)} \phi(x, y) e^{-i \omega m y} \mathrm{~d} y\right| \leq \\
\leq \frac{1}{\omega^{k}} \sum_{|\beta|=k} \frac{k !}{\beta !} \sup _{(x, y) \in \mathbb{R}^{n}}\left|\mathrm{D}^{(\alpha, \beta)} \phi(x, y)\right|=\mathrm{C}(k, \phi) .
\end{gathered}
$$

Observe que $C(k, \phi)$ não depende de $m \in \mathbb{Z}^{\mathrm{q}}$ e que se $\phi_{j} \longrightarrow 0$ em $\mathcal{P}_{\mathrm{T}}\left(\mathbb{R}^{\mathrm{n}}\right)$ então $\mathrm{C}\left(\mathrm{k}, \phi_{\mathrm{j}}\right) \longrightarrow 0$, para cada $\mathrm{k} \in \mathbb{N}$.

\subsection{Série Parcial de Fourier em $\mathcal{P}_{\mathrm{T}}^{\prime}\left(\mathbb{R}^{\mathfrak{n}}\right)$}

Seja $u \in \mathcal{P}_{\mathrm{T}}^{\prime}\left(\mathbb{R}^{p} \oplus \mathbb{R}^{q}\right)$, com $p+q=n$. De maneira análoga ao que foi feito em $\mathcal{P}_{\mathrm{T}}\left(\mathbb{R}^{n}\right)$, exibimos aqui uma sequência de distribuições $\left(u_{m}\right)_{m \in \mathbb{Z}^{q}}, u_{m} \in \mathcal{P}_{T}^{\prime}\left(\mathbb{R}^{p}\right)$, os coeficientes parciais de $u$, tal que podemos escrever

$$
u=\sum_{m \in \mathbb{Z}^{q}} u_{m} e_{m}
$$

Para cada $\gamma \in \mathcal{P}_{\mathrm{T}}\left(\mathbb{R}^{p}\right)$, defina

$$
\begin{aligned}
v_{\gamma}: \mathcal{P}_{\mathrm{T}}\left(\mathbb{R}^{q}\right) & \longrightarrow c \\
\theta & \longmapsto(u, \gamma \otimes \theta)
\end{aligned}
$$

onde

$$
\begin{aligned}
\gamma \otimes \theta: \mathbb{R}^{p} \oplus \mathbb{R}^{q} & \longrightarrow c \\
(x, y) & \longmapsto \gamma(x) \theta(y)
\end{aligned}
$$


Teorema 3.21 Se u $\in \mathcal{P}_{\mathrm{T}}^{\prime}\left(\mathbb{R}^{p} \oplus \mathbb{R}^{q}\right)$ então $\mathrm{u}=\sum_{\mathrm{m} \in \mathbb{Z}^{q}} \mathrm{u}_{\mathrm{m}} e_{\mathrm{m}}$, onde $u_{\mathrm{m}} \in \mathcal{P}_{\mathrm{T}}^{\prime}\left(\mathbb{R}^{p}\right)$ é dada por

$$
\begin{gathered}
\left(u_{\mathrm{m}}, \gamma\right)=\mathrm{c}_{\mathrm{m}}\left(v_{\gamma}\right) \\
\mathrm{c}_{\mathrm{m}}\left(v_{\gamma}\right)=\frac{1}{\mathrm{Tq}}\left(u, \gamma \otimes e_{-m}\right), \gamma \in \mathcal{P}_{\mathrm{T}}\left(\mathbb{R}^{p}\right) .
\end{gathered}
$$

Além do mais, para cada $\gamma \in \mathcal{P}_{\mathrm{T}}\left(\mathbb{R}^{\mathrm{n}}\right),\left(\mathrm{u}_{\mathrm{m}}, \gamma\right)$ é uma sequência de crescimento lento.

DEMONSTRAÇÃO.

$v_{\gamma}$ é linear. Para quaisquer $\theta_{1}, \theta_{2} \in \mathcal{P}_{\mathrm{T}}\left(\mathbb{R}^{\mathrm{q}}\right)$ e $\mathrm{a}_{1}, \mathrm{a}_{2} \in \mathbb{C}$, vale

$$
\begin{gathered}
v_{\gamma}\left(a_{1} \theta_{1}+a_{2} \theta_{2}\right)=\left(u, \gamma \otimes\left(a_{1} \theta_{1}+a_{2} \theta_{2}\right)\right)=\left(u, a_{1} \gamma \otimes \theta_{1}+a_{2} \gamma \otimes \theta_{2}\right)= \\
=a_{1}\left(u, \gamma \otimes \theta_{1}\right)+a_{2}\left(u, \gamma \otimes \theta_{2}\right)=a_{1} v_{\gamma}\left(\theta_{1}\right)+a_{2} v_{\gamma}\left(\theta_{2}\right) .
\end{gathered}
$$

$v_{\gamma}$ é contínuo. Seja $\theta_{j} \longrightarrow 0$ em $\mathcal{P}_{\mathrm{T}}\left(\mathbb{R}^{p}\right)$. Então para todo $(\alpha, \beta) \in \mathbb{N}^{p} \times \mathbb{N}^{q}$, temos

$$
\begin{gathered}
\left|\mathrm{D}^{(\alpha, \beta)} \gamma \otimes \theta_{j}(x, y)\right|=\left|\mathrm{D}^{(\alpha, \beta)} \gamma(x) \theta_{j}(y)\right|= \\
=\left|\mathrm{D}^{\alpha} \gamma(x) \mathrm{D}^{\beta} \theta_{j}(y)\right| \leq \sup \left|\mathrm{D}^{\alpha} \gamma(x)\right| \sup \left|\mathrm{D}^{\beta} \theta_{j}(y)\right| \longrightarrow 0,
\end{gathered}
$$

uniformemente. Logo, $v_{\gamma}\left(\theta_{j}\right)=\left(u, \gamma \otimes \theta_{j}\right) \longrightarrow 0$ em $\mathbb{C}$. Assim, $v_{\gamma} \in \mathcal{P}_{\mathrm{T}}^{\prime}\left(\mathbb{R}^{q}\right)$. Desta forma, $v_{\gamma}=\sum_{m \in \mathbb{Z}^{q}} c_{m}\left(v_{\gamma}\right) e_{m}$, onde

$$
c_{m}\left(v_{\gamma}\right)=\frac{1}{T^{q}}\left(v_{\gamma}, e_{-m}\right)=\frac{1}{T^{q}}\left(u, \gamma \otimes e_{-m}\right) .
$$

Defina, para $m \in \mathbb{Z}^{\mathrm{q}}$,

$$
\begin{aligned}
u_{\mathrm{m}}: \mathcal{P}_{\mathrm{T}}\left(\mathbb{R}^{p}\right) & \longrightarrow \mathbb{C} \\
\gamma & \longmapsto c_{\mathrm{m}}\left(v_{\gamma}\right)
\end{aligned}
$$

$u_{m}$ é linear. De fato, temos

$$
\begin{gathered}
u_{m}\left(a_{1} \gamma_{1}+a_{2} \gamma_{2}\right)=\frac{1}{T q}\left(u,\left(a_{1} \gamma_{1}+a_{2} \gamma_{2}\right) \otimes e_{-m}\right)= \\
=\frac{a_{1}}{T q}\left(u, \gamma_{1} \otimes e_{-m}\right)+\frac{a_{2}}{T q}\left(u, \gamma_{2} \otimes e_{-m}\right)= \\
=a_{1} u_{m}\left(\gamma_{1}\right)+a_{2} u_{m}\left(\gamma_{2}\right),
\end{gathered}
$$

para todos $\gamma_{1}, \gamma_{2} \in \mathcal{P}_{\mathrm{T}}\left(\mathbb{R}^{\mathrm{q}}\right)$ e $a_{1}, a_{2} \in \mathbb{C}$.

$u_{m}$ é contínuo. Seja $\gamma_{j} \longrightarrow 0$ em $\mathcal{P}_{\mathrm{T}}\left(\mathbb{R}^{p}\right)$. Então, para todo $(\alpha, \beta) \in \mathbb{N}^{p} \times \mathbb{N}^{q}$,

$$
\begin{gathered}
\left|D^{(\alpha, \beta)} \gamma_{j} \otimes e_{-m}(x, y)\right|=\left|D^{\alpha} \gamma_{j}(x) D^{\beta} e_{-m}(y)\right| \leq \\
\leq \sup \left|D^{\beta} e_{-m}(y)\right| \sup \left|D^{\alpha} \gamma_{j}(x)\right| \longrightarrow 0
\end{gathered}
$$


uniformemente, quando $j \longrightarrow \infty$, para cada $m \in \mathbb{Z}^{\mathrm{q}}$. Logo,

$$
u_{m}\left(\gamma_{j}\right)=\frac{1}{T q}\left(u, \gamma_{j} \otimes e_{-m}\right) \longrightarrow 0
$$

Assim, $u_{m} \in \mathcal{P}_{\mathrm{T}}^{\prime}\left(\mathbb{R}^{p}\right)$ para todo $m \in \mathbb{Z}^{\mathrm{q}} \mathrm{e}$

$$
\begin{gathered}
v_{\lambda}=\sum_{m \in \mathbb{Z} q}\left(u_{m}, \gamma\right) e_{m} \\
(u, \gamma \otimes \theta)=\left(\sum_{m \in \mathbb{Z} q}\left(u_{m}, \gamma\right) e_{m}, \theta\right) .
\end{gathered}
$$

Note que esta expressão já é suficiente para que $u$ fique bem definida, bastando tomar $\gamma(x)=e^{-i \omega m_{1} x \mathfrak{s}}$ e $\theta(y)=e^{-i \omega m_{2} y}$, onde $m=m_{1}+m_{2} \in \mathbb{Z}^{n}, m_{1} \in \mathbb{Z}^{p}$ e $m_{2} \in \mathbb{Z}^{q}$. Daí,

$$
c_{m}(u)=(u, \gamma \otimes \theta)
$$

Vejamos como é o crescimento da sequência $\left(u_{m}\right)_{m} \in \mathbb{Z}^{q}$. Como $u \in \mathcal{P}_{T}^{\prime}\left(\mathbb{R}^{p+q}\right)$, existem $\mathrm{C}_{0}>0$ e $\mathrm{k}_{0} \in \mathbb{N}$ tais que, para todo $\gamma \in \mathcal{P}_{\mathrm{T}}\left(\mathbb{R}^{\mathrm{p}}\right)$,

$$
\begin{gathered}
\left|\left(u_{m}, \gamma\right)\right|=\frac{1}{T q}\left|\left(u, \gamma \otimes e_{-m}\right)\right| \leq C_{0} \sum_{|(\alpha, \beta)| \leq k_{0}}\left|D^{(\alpha, \beta)}\left(\gamma(x) e_{-m}(y)\right)\right|= \\
=C_{0} \sum_{|(\alpha, \beta)| \leq k_{0}} \sup \left|D^{\alpha} \gamma(x)(-i \omega m)^{\beta} e^{-i \omega m y}\right|= \\
=C_{0} \sum_{|(\alpha, \beta)| \leq k_{0}} \sup \left|D^{\alpha} \gamma(x)\right|\left|(\omega m)^{\beta}\right| .
\end{gathered}
$$

Então, para algum $C_{1}>0$,

$$
\left|\left(u_{m}, \gamma\right)\right| \leq C_{1} p_{k_{0}}(\gamma)(1+|m|)^{k_{0}},
$$

onde

$$
p_{k_{0}}(\gamma)=\sum_{|\alpha| \leq k_{0}} \sup \left|D^{\alpha} \gamma\right|
$$

Teorema 3.22 Seja $\left(u_{m}\right)_{\mathfrak{m} \in \mathbb{Z} q}$ uma sequência de $\mathcal{P}_{T}^{\prime}\left(\mathbb{R}^{p}\right)$ que satisfaz a seguinte condição: existe $\mathrm{C}>0$ e $\mathrm{k}_{0} \in \mathbb{N}$ tais que

$$
\left|\left(u_{m}, \phi\right)\right| \leq C p_{k_{0}}(\phi)(1+|m|)^{k_{0}},
$$

para toda $\phi$ de $\mathcal{P}_{\mathrm{T}}\left(\mathbb{R}^{p}\right)$ e todo $\mathrm{m}$ de $\mathbb{Z}^{\mathrm{q}}$.

Então $u=\sum_{m \in \mathbb{Z} q} u_{m} e_{m}$ pertence $a \mathcal{P}_{\mathrm{T}}^{\prime}\left(\mathbb{R}^{p+q}\right)$. 
DemonstraçÃo. Para cada $\theta \in \mathcal{P}_{\mathrm{T}}\left(\mathbb{R}^{\mathrm{p}+\mathrm{q}}\right)$, considere a sequência

$$
\begin{gathered}
s_{j}=\sum_{\substack{|m| \leq j \\
m \in \mathbb{Z} q}}\left(u_{m}(x) e_{m}(y), \theta(x, y)\right)= \\
=\sum_{\substack{|m| \leq j \\
m \in \mathbb{Z} q}}\left(u_{m}(x), \int_{\substack{[0, T] q \\
m}} e_{m}(y) \theta(x, y) d y\right)=\sum_{\substack{|m| \leq j \\
m \in \mathbb{Z} q}}\left(u_{\mathfrak{m}}(x), \theta_{\mathfrak{m}}(x)\right) .
\end{gathered}
$$

Para cada $\ell \in \mathbb{N}$, vale que

$$
\left|s_{j+\ell}-s_{j}\right| \leq \sum_{\substack{j<|m| \leq j+\ell \\ m \in \mathbb{Z} q}}\left|\left(u_{m}, \theta_{m}\right)\right| \leq C \sum_{\substack{j<|m| \leq j+\ell \\ m \in \mathbb{Z} q}} p_{k_{0}}\left(\theta_{m}\right)(1+|m|)^{k_{0}} .
$$

Porém, como visto, $\theta_{\mathrm{m}}$ é rapidamente decrescente, sendo assim,

$$
\begin{gathered}
p_{k_{0}}\left(\theta_{m}\right)=\sum_{\substack{|\alpha| \leq k_{0} \\
\alpha \in \mathbb{N} p}} \sup \left|D^{\alpha} \theta_{m}(x)\right| \leq \\
\leq \sum_{\substack{|\alpha| \leq k_{0} \\
\alpha \in \mathbb{N} \mathbb{P}}} C_{\alpha}(1+|m|)^{-k_{0}-q-1} \leq C^{\prime}(1+|m|)^{-k_{0}-q-1} .
\end{gathered}
$$

onde os $C_{\alpha}$ 's vêm do teorema 3.19 e $C^{\prime}=\max _{|\alpha| \leq k_{0}} C_{\alpha}$. Assim,

$$
\left|s_{j+p}-s_{j}\right| \leq C^{\prime} \sum_{\substack{j<|m| \leq j+p \\ m \in \mathbb{Z} q}}(1+|m|)^{-q-1} \longrightarrow 0,
$$

quando $j \longrightarrow \infty$. Deste modo, $s_{j}$ é convergente e $\sum_{m \in Z q} u_{m} e_{m}$ pertence a $\mathcal{P}_{T}^{\prime}\left(\mathbb{R}^{p+q}\right)$. 


\section{Capítulo 4}

\section{Funções Analíticas Reais em $\mathbb{R}^{n}$}

Este capítulo contém fatos iniciais à respeito de funções analíticas reais de várias variáveis, que são funções que podem ser representadas por uma série de potências de várias variáveis, em cada ponto de seu domínio. São analisados elementos básicos de uma série de potências. $\mathrm{N}$ o caso de várias variáveis, conceitos simples como convergência e domínio de convergência adquirem um caráter mais variado. Mostramos que o domínio de convergência de uma série de potências em $\mathbb{R}^{n}$ é um conjunto logaritmicamente convexo.

Em seguida apresentamos alguns fatos a respeito de funções analíticas reais, essenciais para um entendimento autocontido de nosso objetivo. Por fim apresentamos um teorema muito recorrido na obtenção do resultado principal, devido à Bergamasco,que caracteriza o decaimento dos coeficientes parciais de Fourier de uma função analítica real periódica.

Faremos uso das notações de múlti-índices introduzidas na definição 1.2. Fizemos uso das referências $[\mathrm{C}]$ e $[\mathrm{KP}]$.

\subsection{Série de Potências de Várias Variáveis}

Definição 4.1 Sejam $c \in \mathbb{R}^{n} e\left(a_{\alpha}\right)_{\alpha \in \mathbb{N}^{n}}, a_{\alpha} \in \mathbb{R}^{n}$, uma sequência indexada em $\mathbb{N}^{n}$. Uma série de potências de $\mathrm{n}$ variáveis é uma expressão da forma

$$
\sum_{\alpha \in \mathbb{N}^{n}} a_{\alpha}(x-c)^{\alpha}
$$

Definição 4.2 Dizemos que a série de potências $\sum_{\alpha \in \mathbb{N}^{n}} a_{\alpha}(x-c)^{\alpha}$ converge em $x_{0} \in \mathbb{R}^{n}$ quando o conjunto

$$
S\left(x_{0}\right)=\left\{\sum_{\alpha \in \Lambda}\left|a_{\alpha}\right|\left|x_{0}-c\right|^{\alpha}: \Lambda \subset \mathbb{N}^{n}, \Lambda \text { é finito }\right\}
$$

é um subconjunto limitado de $\mathbb{R}$. 
Teorema 4.3 Seja $\sum_{\alpha \in \mathbb{N}^{n}} a_{\alpha}(x-c)^{\alpha}$ uma série de potências convergente em $x_{0}$. Então existe um único $\ell \in \mathbb{R}^{n}$, tal que para todo $\varepsilon>0$, existe $N \in \mathbb{N}$, tal que $\mathrm{m} \geq \mathrm{N}$ implica que

$$
\left|\sum_{|\alpha| \leq m} a_{\alpha}\left(x_{0}-c\right)^{\alpha}-\ell\right|<\varepsilon .
$$

DemonstraçÃo. Note que

$$
\sum_{|\alpha| \leq m} a_{\alpha}\left(x_{0}-c\right)^{\alpha}=\sum_{k=0}^{m} \sum_{|\alpha|=k} a_{\alpha}\left(x_{0}-c\right)^{\alpha} \doteq S_{m} .
$$

Seja $\gamma=\sup S\left(x_{0}\right)$. Então dado $\varepsilon>0$, existe $\Lambda \subset \mathbb{N}^{n}, \Lambda$ finito, tal que

$$
\gamma-\varepsilon<\sum_{\alpha \in \Lambda}\left|a_{\alpha}\right|\left|x_{0}-c\right|^{\alpha} \leq \gamma
$$

Seja $N_{1}=\max \{|\alpha|: \alpha \in \Lambda\}$. Se $m \geq N_{1}$ então

$$
\gamma-\varepsilon<\sum_{\alpha \in \Lambda}\left|a_{\alpha}\right|\left|x_{0}-c\right|^{\alpha} \leq \sum_{|\alpha| \leq m}\left|a_{\alpha} \| x_{0}-c\right|^{\alpha} \leq \gamma
$$

Agora, se $p>m \geq N_{1}$ então

$$
\begin{aligned}
& \left|S_{p}-S_{m}\right|=\left|\sum_{|\alpha| \leq p} a_{\alpha}\left(x_{0}-c\right)^{\alpha}-\sum_{|\alpha| \leq m} a_{\alpha}\left(x_{0}-c\right)^{\alpha}\right|= \\
= & \left|\sum_{k=m+1}^{p} \sum_{|\alpha|=k} a_{\alpha}\left(x_{0}-c\right)^{\alpha}\right| \leq \sum_{k=m+1}^{p} \sum_{|\alpha|=k}\left|a_{\alpha}\right|\left|x_{0}-c\right|^{\alpha}= \\
= & \sum_{|\alpha| \leq p}\left|a_{\alpha}\right|\left|x_{0}-c\right|^{\alpha}-\sum_{|\alpha| \leq m}\left|a_{\alpha}\right|\left|x_{0}-c\right|^{\alpha}<\gamma-(\gamma-\varepsilon)=\varepsilon .
\end{aligned}
$$

Dessa maneira, concluímos que $S_{m}$ é uma sequência de Cauchy em $\mathbb{R}^{n}$ e, portanto, existe um único $\ell \in \mathbb{R}^{n}$ tal que dado $\varepsilon>0$ existe $N \in \mathbb{N}$ tal que $m \geq N$ implica que

$$
\left|\sum_{|\alpha| \leq m} a_{\alpha}\left(x_{0}-c\right)^{\alpha}-\ell\right|<\varepsilon .
$$

Nas condições do teorema acima, faz sentido escrevermos

$$
\sum_{\alpha \in \mathbb{N}^{n}} a_{\alpha}\left(x_{0}-c\right)^{\alpha}=\ell
$$

quando a série for convergente. 
Observação $4.4 S e \sum_{\alpha \in \mathbb{N}^{n}} a_{\alpha}\left(x_{0}-c\right)^{\alpha}$ converge então

$$
\sum_{\alpha \in \mathbb{N}^{n}}\left|a_{\alpha}\right|\left|x_{0}-c\right|^{\alpha}=\gamma
$$

onde $\gamma$ é como na prova do teorema acima.

Teorema 4.5 Seja $\sum_{\alpha \in \mathbb{N}^{n}} a_{\alpha}(x-c)^{\alpha}$ uma série de potências convergente em $x_{0}$. Então existe um único $\ell \in \mathbb{R}^{n}$, tal que para todo $\varepsilon>0$, existe $\mathrm{N} \in \mathbb{N}$, tal que, se $\Lambda \subset \mathbb{N}^{n}$ é um conjunto finito, $e\{\alpha:|\alpha| \leq \mathrm{N}\} \subset \Lambda$, então

$$
\left|\sum_{\alpha \in \Lambda} a_{\alpha}\left(x_{0}-c\right)^{\alpha}-\ell\right|<\varepsilon
$$

Demonstração. Pelo teorema anterior existe $\ell \in \mathbb{R}^{n}$ tal que dado $\varepsilon>0$, existe $N_{1}>0$ tal que $m \geq N_{1}$ implica em

$$
\left|\sum_{|\alpha| \leq m} a_{\alpha}\left(x_{0}-c\right)^{\alpha}-\ell\right|<\frac{\varepsilon}{2} .
$$

Seja $\gamma$ como na prova do teorema anterior. Pela definição de supremo, segue que existe $\mathrm{N}_{2} \in \mathbb{N}$ tal que $\mathrm{m} \geq \mathrm{N}_{2}$ implica em

$$
\gamma-\frac{\varepsilon}{2} \leq \sum_{|\alpha| \leq m}\left|a_{\alpha}\right|\left|x_{0}-c\right|^{\alpha} \leq \gamma .
$$

Defina $N=\max \left\{N_{1}, N_{2}\right\}$. Seja $\Lambda$ um conjunto finito tal que $\{\alpha:|\alpha| \leq N\} \subset \Lambda$. Considere $M=\max \{|\alpha|: \alpha \in \Lambda\} \geq N$. Com isso,

$$
\begin{gathered}
\left|\sum_{\alpha \in \Lambda} a_{\alpha}\left(x_{0}-c\right)^{\alpha}-\ell\right|=\left|\sum_{|\alpha| \leq N} a_{\alpha}\left(x_{0}-c\right)^{\alpha}-\ell+\sum_{\substack{\alpha \in \Lambda \\
|\alpha|>N}} a_{\alpha}\left(x_{0}-c\right)^{\alpha}\right| \leq \\
\leq\left|\sum_{|\alpha| \leq N} a_{\alpha}\left(x_{0}-c\right)^{\alpha}-\ell\right|+\sum_{N<|\alpha| \leq M}\left|a_{\alpha}\right|\left|x_{0}-c\right|^{\alpha}<\frac{\varepsilon}{2}+\frac{\varepsilon}{2}=\varepsilon .
\end{gathered}
$$

Para ver que $\ell$ é único, note que se existisse $\ell_{1}$ com a mesma propriedade que $\ell$, então $\ell_{1}$ satisfaz as condições do teorema 4.3. Logo, $\ell_{1}=\ell$.

Teorema 4.6 Se $\sum_{\alpha \in \mathbb{N}^{n}} a_{\alpha}\left(x_{0}-c\right)^{\alpha}=\ell$ e $\sigma: \mathbb{N} \longrightarrow \mathbb{N}^{n}$ é uma bijeção então

$$
\sum_{k=0}^{\infty} a_{\sigma(k)}\left(x_{0}-c\right)^{\sigma(k)}=\ell .
$$


Demonstração. Dado $\varepsilon>0$, tome $\mathrm{N}$ como na prova do teorema anterior. Temos que $\sigma^{-1}(\{\alpha:|\alpha| \leq N\}) \subset \mathbb{N}$ é finito. Seja $N_{1}=\max \sigma^{-1}(\{\alpha:|\alpha| \leq N\})$. Se $m \geq N_{1}$, seja $\Lambda=\{\sigma(1), \ldots, \sigma(m)\}$. Com isso,

$$
\{\alpha:|\alpha| \leq N\} \subset\left\{\sigma(1), \ldots, \sigma\left(N_{1}\right)\right\} \subset\{\sigma(1), \ldots, \sigma(m)\}=\Lambda .
$$

Pelo teorema anterior concluímos que

$$
\left|\sum_{k=0}^{m} a_{\sigma(k)}\left(x_{0}-c\right)^{\sigma(k)}-\ell\right|=\left|\sum_{\alpha \in \Lambda} a_{\alpha}\left(x_{0}-c\right)^{\alpha}-\ell\right|<\varepsilon .
$$

Observação 4.7 Considere a série de potências $\sum_{\alpha \in \mathbb{N}^{n}} a_{\alpha}(x-c)^{\alpha}$. Seja $x_{0} \in \mathbb{R}^{n}$. Se existem $\mathrm{L}>0, \mathrm{~N} \in \mathbb{N}$, tais que $\mathrm{m} \geq \mathrm{N}$ implica

$$
\sum_{|\alpha| \leq m}\left|a_{\alpha}\right|\left|x_{0}-c\right|^{\alpha} \leq L
$$

então a série é convergente em $x_{0}$.

O próximo lema é necessário à demonstração do lema de Abel, que veremos a seguir.

Lema 4.8 Sejam $\mathrm{n} \geq 1$ e $\mathrm{m} \geq 0$ números inteiros $e-1<\mathrm{t}<1$ um número real. Temos que

1. $\sum_{j=0}^{m}\left(\begin{array}{c}j+n-1 \\ n-1\end{array}\right)=\left(\begin{array}{c}m+n \\ n\end{array}\right)$

2. $\#\left\{\alpha \in \mathbb{N}^{n}:|\alpha|=m\right\}=\left(\begin{array}{c}m+n-1 \\ n-1\end{array}\right)$

3. $\sum_{j=0}^{\infty}\left(\begin{array}{c}m+j \\ m\end{array}\right) j ! t^{j}=\frac{\partial^{m}}{\partial t^{m}}\left(\frac{t^{m}}{1-t}\right)$

\section{DEMONSTRAÇÃO.}

1. Defina $P(m)=\left(\sum_{j=0}^{m}\left(\begin{array}{c}j+n-1 \\ n-1\end{array}\right)=\left(\begin{array}{c}m+n \\ n\end{array}\right)\right.$, para todo $\left.n \geq 1\right)$. Devemos mostrar por indução, que $P(m)$ é verdadeiro para todo $m \in \mathbb{N}$. Basta notar que se $P(k)$ é verdade, então $\sum_{j=0}^{k+1}\left(\begin{array}{c}j+n-1 \\ n-1\end{array}\right)=\sum_{j=0}^{k}\left(\begin{array}{c}j+n-1 \\ n-1\end{array}\right)+\left(\begin{array}{c}k+n \\ n-1\end{array}\right)=\left(\begin{array}{c}k+m \\ n\end{array}\right)+\left(\begin{array}{c}k+n \\ n-1\end{array}\right)=\left(\begin{array}{c}k+1+n \\ n\end{array}\right)$.

2. Defina $\mathrm{Q}(\mathrm{m})=\left(\#\left\{\alpha \in \mathbb{N}^{n}:|\alpha|=\mathrm{m}\right\}=\left(\begin{array}{c}\mathrm{m}+\mathrm{n}-1 \\ \mathrm{n}-1\end{array}\right)\right.$, para todo $\left.\mathrm{m} \in \mathbb{N}\right)$. Para obtermos que $Q(k+1)$ verdadeiro, sabendo que $Q(k)$ verdadeiro, basta notar que o número de $(k+1)$-uplas com soma $m$, igual ao número de $k+1$-uplas cuja primeira coordenada 0 , com soma das coordenadas restantes sendo $\mathrm{m}$, mais o número de $(k+1)$-uplas cuja primeira coordenada 1 , com soma das coordenadas restantes sendo $m-1, \ldots$, mais o número de $(k+1)$-uplas cuja primeira coordenada $m$, com a soma das coordenadas restantes sendo 0 . 
3. Basta notar que $\frac{\mathrm{t}^{\mathrm{m}}}{1-\mathrm{t}}=\mathrm{t}^{\mathrm{m}} \sum_{j=0}^{\infty} \mathrm{t}^{j}=\sum_{j=0}^{\infty} \mathrm{t}^{j+\mathrm{m}}$, e que podemos derivar a série em questão, termo a termo.

Definição 4.9 Seja $x=\left(x_{1}, \ldots, x_{n}\right) \in \mathbb{R}^{n}$. A silhueta de $x$ é o conjunto

$$
s(x)=\left\{\left(r_{1} x_{1}, \ldots, r_{n} x_{n}\right):-1<r_{j}<1, j=1, \ldots, n\right\} .
$$

Lema 4.10 (Abel) Se a série de potências $\sum_{\alpha \in \mathbb{N}^{n}} a_{\alpha} x^{\alpha}$ converge em $x=\left(x_{1}, \ldots, x_{n}\right)$ então a série converge uniforme e absolutamente em qualquer subconjunto compacto de $\mathrm{s}(\mathrm{x})$.

Demonstração. Seja $K$ um subconjunto compacto de $s(x)$. Afirmamos que existe $0<\rho<1$ tal que $\left|k_{j}\right| \leq \rho\left|x_{j}\right|$, para todo $k=\left(k_{1}, \ldots, k_{n}\right) \in K, j=1, \ldots, n$.

Vamos mostrar que a afirmação vale para $j=1$. Suponha por absurdo, que para todo $0<\sigma<1$, existe $k(\sigma) \in K$ tal que $\left|k_{1}(\sigma)\right|>\sigma\left|x_{1}\right|$. Desta forma, podemos tomar $\sigma_{\mathrm{m}}=1-\mathrm{m}^{-1}, \mathrm{~m}=2,3, \ldots$, para concluir que

$$
\left|x_{1}\right|>\left|k_{1}\left(\sigma_{n}\right)\right|>\sigma_{n}\left|x_{1}\right|,
$$

para $m=2,3, \ldots$. Com isso, concluímos que $\left|x_{1}\right|$ é um ponto de acumulação do conjunto compacto $\left\{\left|\mathrm{k}_{1}\right| ; \mathrm{k} \in \mathrm{K}\right\}$, que não pertence ao mesmo, absurdo. Portanto, existe $0<\sigma_{1}<1$, tal que $\left|k_{1}\right| \leq \sigma_{1}\left|x_{1}\right|$. De maneira análoga, obtemos $\sigma_{2}, \ldots, \sigma_{n}$, para definirmos $\rho=\max \left\{\sigma_{1}, \ldots, \sigma_{n}\right\}$.

Como a série converge em $x$, existe uma constante $C>0$ tal que $\left|a_{\alpha} \| x\right|^{\alpha} \leq C$ para todo $\alpha \in \mathbb{N}^{n}$, o que implica em $\left|a_{\alpha}\right||k|^{\alpha} \leq C \rho^{|\alpha|}, \alpha \in \mathbb{N}^{n}, k \in K$. Usando agora o lema 4.8 e a observação 4.7 , temos que

$$
\begin{gathered}
\sum_{|\alpha| \leq N}\left|a_{\alpha}\right||k|^{\alpha}=\sum_{j=0}^{N} \sum_{|\alpha|=j}\left|a_{\alpha}\right||k|^{\alpha} \leq \sum_{j=0}^{N} \sum_{|\alpha|=j} C \rho^{|\alpha|} \\
\leq \sum_{j=0}^{N} C\left(\begin{array}{c}
n+j-1 \\
n-1
\end{array}\right) \rho^{j} \leq \sum_{j=0}^{\infty} C\left(\begin{array}{c}
n+j-1 \\
n-1
\end{array}\right) \rho^{j} j !=C^{(n-1)}(\rho),
\end{gathered}
$$

onde $h(t)=\frac{t^{n-1}}{1-t}$. Como $C$ independente de $k$ e $N$, concluímos a demonstração.

Definição 4.11 Dada a série de potências $\sum_{\alpha \in \mathbb{N}^{n}} a_{\alpha} \chi^{\alpha}$, considere o conjunto

$$
\mathcal{C}^{\prime}=\left\{x \in \mathbb{R}^{n}: \sum_{\alpha \in \mathbb{N}^{n}} a_{\alpha} x^{\alpha} \text { é convergente }\right\} l
$$

Defina o dominio de convergência da série de potências como sendo $\mathcal{C}=\operatorname{Int} \mathcal{C}^{\prime}$ 
Proposição 4.12 Seja $\mathcal{B}=\left\{x ; \exists C>0,\left|a_{\alpha}\right||x|^{\alpha} \leq C, \forall \alpha \in \mathbb{N}^{n}\right\}$. Então $\mathcal{C}=$ Int $\mathcal{B}$.

Demonstração. $(\mathcal{C} \subset \operatorname{Int} \mathcal{B})$ Se $x \in \mathcal{C}$ então existe $\varepsilon>0$ tal que, se $E=\left(\tau_{1}, \ldots, \tau_{n}\right)$, $\left|\tau_{j}\right| \leq \varepsilon, j=1, \ldots, n$, então $\sum_{\alpha \in \mathbb{N}^{n}} a_{\alpha}(x+E)^{\alpha}$ é convergente. Portanto, existe $C_{E}>0$ tal que $\left|a_{\alpha} \|(x+E)^{\alpha}\right| \leq C_{E}$, para todo $\alpha \in \mathbb{N}^{n}$.

(Int $\mathcal{B} \subset \mathcal{C}$ ) Considere a demonstração do lema de Abel. Para cada $x \in \operatorname{Int} \mathcal{B}$, existem $0<\rho<1$ e $y \in \mathcal{B}$ tais que $\left|x_{j}\right| \leq \rho\left|y_{j}\right|, j=1, \ldots, n$. Além disto, existe $D>0$ tal que $\left|a_{\alpha}\right||y|^{\alpha} \leq D$, para todo $\alpha \in \mathbb{N}^{m}$. Daqui em diante, procedemos assim como na demonstração do lema de Abel para concluir que $\sum_{\alpha \in \mathbb{N}^{n}} a_{\alpha} \chi^{\alpha}$ é convergente e o mesmo vale se $x+E \in \mathcal{B}, E=\left(\tau_{1}, \ldots, \tau_{n}\right),\left|\tau_{j}\right| \leq \varepsilon$ para algum $\varepsilon>0$ conveniente.

Definição 4.13 Seja $S$ um conjunto tal que $S \subset \mathbb{R}^{n}$, definimos

$$
\log \|S\|=\left\{\left(\log \left|s_{1}\right|, \ldots, \log \left|s_{n}\right|\right) ; s=\left(s_{1}, \ldots, s_{n}\right) \in S\right\}
$$

O conjunto $S$ é chamado logaritmicamente convexo se log $\|S\|$ é um subconjunto convexo de $\mathbb{R}^{n}$.

Proposição 4.14 Seja $\sum_{\alpha \in \mathbb{N}^{m}} a_{\alpha} \chi^{\alpha}$ uma série de potências. Seu domínio de convergência é logaritmicamente convexo.

Demonstração. Fixe $y, z \in \mathcal{C}=$ Int $B$, e $0 \leq \lambda \leq 1, y=\left(y_{1}, \ldots, y_{n}\right)$ e $z=$ $\left(z_{1}, \ldots, z_{\mathfrak{n}}\right)$. Como $z, y \in \mathcal{C}$, existem $C>0, \varepsilon>0$ tais que

$$
\begin{aligned}
& \left|a_{\alpha}\right| \leq \frac{C}{\prod_{j=1}^{n}\left(\left|y_{j}\right|+\varepsilon\right)^{\alpha_{j}}} \\
& \left|a_{\alpha}\right| \leq \frac{C}{\prod_{j=1}^{n}\left(\left|z_{j}\right|+\varepsilon\right)^{\alpha_{j}}} .
\end{aligned}
$$

Podemos escolher $\varepsilon^{\prime}>0$, tal que $\left|y_{j}+\varepsilon\right|^{\lambda} \geq\left|y_{j}\right|^{\lambda}+\varepsilon^{\prime}$, e $\left|z_{j}+\varepsilon\right|^{\lambda} \geq\left|z_{j}\right|^{1-\lambda}+\varepsilon^{\prime}$, para $j=1, \ldots, n$. Também podemos escolher $\sigma>0$, tal que

$$
\left(\left|y_{j}\right|^{\lambda}+\varepsilon^{\prime}\right)\left(\left|z_{j}\right|^{\lambda}+\varepsilon^{\prime}\right) \geq\left|y_{j}\right|^{\lambda}\left|z_{j}\right|^{1-\lambda}+\sigma,
$$

para todo $j=1, \ldots, n$. Com isso, concluímos que

$$
\begin{aligned}
\left|a_{\alpha}\right| & =\left|a_{\alpha}\right|^{\lambda}\left|a_{\alpha}\right|^{1-\lambda} \leq \frac{C^{\lambda}}{\prod_{j=1}^{n}\left(\left|y_{j}\right|+\varepsilon\right)^{\alpha_{j} \lambda}} \frac{C^{1-\lambda}}{\prod_{j=1}^{n}\left(\left|z_{j}\right|+\varepsilon\right)^{\alpha_{j}(1-\lambda)}} \leq \\
& \leq \frac{C}{\prod_{j=1}^{n}\left(\left|y_{j}\right|^{\lambda}\left|z_{j}\right|^{1-\lambda}+\sigma\right)^{\alpha_{j}}} \leq \frac{C}{\prod_{j=1}^{n}\left(\left|y_{j}\right|^{\lambda}\left|z_{j}\right|^{1-\lambda}+\sigma^{\prime}\right)^{\alpha_{j}}},
\end{aligned}
$$


pra todo $\sigma^{\prime} \leq \sigma$ e, desta forma, obtemos

$$
\left(\left|y_{1}\right|^{\lambda}\left|z_{1}\right|^{1-\lambda}, \ldots,\left|y_{m}\right|^{\lambda}\left|z_{m}\right|^{1-\lambda}\right) \in \operatorname{Int} \mathcal{B}=\mathcal{C}
$$

e com isso

$$
\left(\log \left(\left|y_{1}\right|^{\lambda}\left|z_{1}\right|^{1-\lambda}\right), \ldots, \log \left(\left|y_{n}\right|^{\lambda}\left|z_{n}\right|^{1-\lambda}\right)\right) \in \log \|\mathcal{C}\|
$$

o que nos dá

$$
\lambda\left(\log \left|y_{1}\right|, \ldots, \log \left|y_{n}\right|\right)+(1-\lambda)\left(\log \left|z_{1}\right|, \ldots, \log \left|z_{n}\right|\right) \in \log \|\mathcal{C}\|
$$

e portanto $\mathcal{C}$ é logaritmicamente convexo.

\subsection{Funções Analíticas Reais}

Definição 4.15 Seja $\mathrm{f}: \mathrm{U} \rightarrow \mathbb{C}$, onde $\mathrm{U} \subset \mathbb{R}^{n}$ é um aberto. Dizemos que $\mathrm{f}$ é analítica real se para cada $x_{0}$ em $\mathrm{U}$ a função $f$ pode ser representada por uma série de potências de várias variáveis, num aberto $\mathrm{V} \subset \mathrm{U}$ contendo $x_{0} . O$ conjunto de tais funções será denotado por $\mathrm{C}^{\omega}(\mathrm{U})$.

Proposição 4.16 Seja $\mathrm{f}: \mathrm{U} \rightarrow \mathbb{C}$, onde $\mathrm{U} \subset \mathbb{R}^{n}$ é um aberto. Então $\mathrm{f}$ é analítica real se e somente se existem um aberto $\mathrm{W} \subset \mathbb{C}^{n}, \mathrm{U} \subset \mathrm{W}$ e uma função holomorfa (analítica) $\mathrm{F}: \mathrm{W} \longrightarrow \mathbb{C}$ tal que $\mathrm{F}_{\mid \mathrm{u}}=\mathrm{f}$.

DEMONSTRAÇÃo. $(\Longrightarrow)$ Se f é analítica real então, para todo $c \in U$, existe $B_{\mathbb{R}^{n}}\left(c, r_{c}\right)$ tal que $f(x)=\sum_{\alpha \in \mathbb{N}^{n}} a_{\alpha}(c)(x-c)^{\alpha}$, para todo $x \in B_{\mathbb{R}^{n}}\left(c, r_{c}\right)$. Note agora que se $z=\left(x_{1}+i y_{1}, \ldots, x_{n}+i y_{n}\right), z \in \mathbb{C}^{n}$, tal que $z \in B_{\mathbb{C}^{n}}\left(c, r_{c}\right)$, então faz sentido falarmos que a série $\sum_{\alpha \in \mathbb{N}^{n}} a_{\alpha}(c)(z-c)^{\alpha}$ converge em $B_{\mathbb{C}^{n}}\left(c, r_{c}\right)$, segundo a definição 4.2. Defina $W=\cup_{c \in U} B_{\mathbb{C}^{n}}\left(c, r_{c}\right)$. Para definirmos $F$ em $z \in W$, tome $c \in U$ tal que $z \in B_{\mathbb{C}}\left(c, r_{c}\right)$ e coloque $F(z)=\sum_{\alpha \in \mathbb{N}^{n}} a_{\alpha}(c)(z-c)^{\alpha}$. Para ver que $F$ está bem definida, observe que se existirem $c_{1}, c_{2}$ tais que $z \in B_{\mathbb{C}^{n}}\left(c_{1}, r_{1}\right) \cap z \in B_{\mathbb{C}^{n}}\left(c_{2}, r_{2}\right)$ então, colocando

$$
\begin{aligned}
& \mathrm{f}_{\mathrm{c}_{1}}(z)=\sum_{\alpha \in \mathbb{N}^{n}} \mathrm{a}_{\alpha}\left(\mathrm{c}_{1}\right)\left(z-\mathrm{c}_{1}\right)^{\alpha}, z \in \mathrm{B}_{\mathbb{C}^{n}}\left(\mathrm{c}_{1}, \mathrm{r}_{1}\right), \\
& \mathrm{f}_{\mathrm{c}_{2}}(z)=\sum_{\alpha \in \mathbb{N}^{n}} \mathrm{a}_{\alpha}\left(\mathrm{c}_{2}\right)\left(z-\mathrm{c}_{2}\right)^{\alpha}, z \in \mathrm{B}_{\mathbb{C}^{n}}\left(\mathrm{c}_{2}, \mathrm{r}_{2}\right)
\end{aligned}
$$

e I $=B_{\mathbb{C}^{n}}\left(c_{1}, r_{1}\right) \cap B_{\mathbb{C}^{n}}\left(c_{2}, r_{2}\right)$ temos $f_{c_{1}}(z)=f_{c_{2}}(z), \forall z \in$ I. De fato, seja $z=$ $\left(z_{1}, \ldots, z_{n}\right)=\left(x_{1}+i y_{1}, \ldots, x_{n}+i y_{n}\right), z \in I$. Vamos mostrar, que $f_{c_{1}}(z)=f_{c_{2}}(z), \forall z \in$ I. Defina $\tilde{f_{c_{1}}}\left(z_{1}\right)=f_{c_{1}}\left(z_{1}, z_{2}, \ldots, z_{n}\right), \quad \tilde{f_{c_{2}}}\left(z_{2}\right)=$ $f_{c_{2}}\left(z_{1}, z_{2}, \ldots, z_{n}\right)$. Note que $\tilde{f}_{c_{1}}$ e $\tilde{f}_{c_{1}}$ são funções holomorfas em $\mathrm{I} \cap \mathbb{C}$ e coincidem 
em $I \cap \mathbb{R}$. Portanto, $\tilde{f}_{c_{1}}=\tilde{f}_{c_{2}}$, em $I \cap \mathbb{C}$. Segue disso que $f_{c_{1}}(z)=f_{c_{2}}(z), \forall z \in I$ e, portanto, podemos definir $F(z)=f_{c_{1}}(z)$.

$(\Longleftarrow)$ Se existem um aberto $W \subset \mathbb{C}^{n}, \mathrm{U} \subset \mathrm{W}$ e uma função holomorfa $\mathrm{F}: \mathrm{W} \longrightarrow \mathbb{C}$ tal que $F_{\mid u}=f$, então $f$ é uma série de potências e o resultado segue da proposição 4.21 .

Seja $\beta \in \mathbb{N}^{n}$. Se a série de potências

$$
\sum_{\alpha \in \mathbb{N}^{n}} a_{\alpha}(x-c)^{\alpha}
$$

é derivada termo a termo por $\frac{\partial^{\beta}}{\partial x^{\beta}}$, obtemos a série

$$
\sum_{\alpha \in \mathbb{N}^{n}}(\alpha+\beta)_{\beta} a_{\alpha+\beta}(x-c)^{\alpha} .
$$

Vamos agora usar a série derivada, para mostrar que uma função analítica real é derivável.

Proposição 4.17 Seja f uma função analítica real definida no aberto $\mathrm{U} \subset \mathbb{R}^{\mathrm{n}}$. Então f é contínua, e possui derivadas parciais continuas e analiticas reais, de todas as ordens.

DemonstraçÃo. Numa vizinhança apropriada de $c \in U$, temos que $f$ é dada por

$$
\sum_{\alpha \in \mathbb{N}^{n}} a_{\alpha}(x-c)^{\alpha} .
$$

Tome $T>0$ tal que a série converge em $c+t$, com $t=(T, \ldots, T) \in \mathbb{R}^{n}$. Dessa maneira, existe uma constante $C$ tal que $\left|a_{\alpha}\right| T^{|\alpha|} \leq C$. Escolha $0<\rho<1$ e considere $x$ tal que $\left|x_{i}-c_{i}\right| \leq \rho T, j=1, \ldots, n$. Para a série derivada, vale a desigualdade

$$
\begin{gathered}
\sum_{\alpha \in \mathbb{N}^{n}}(\alpha+\beta)_{\beta}\left|a_{\alpha+\beta}\right||x-c|^{\alpha} \leq C \sum_{\alpha \in \mathbb{N}^{n}}(\alpha+\beta)_{\beta} \frac{|x-c|^{\alpha}}{T^{|\alpha|+|\beta|}}= \\
=\frac{C}{T^{\beta}} \sum_{j=0}^{\infty} \sum_{|\alpha|=j}(\alpha+\beta)_{\beta} \frac{|x-c|^{\alpha}}{T^{|\alpha|}} \leq \frac{C}{T^{\beta}} \sum_{j=0}^{\infty}(j+|\beta|)_{|\beta|}\left(\begin{array}{c}
m+j-1 \\
m-1
\end{array}\right) \rho^{j},
\end{gathered}
$$

onde a última série converge, pelo teste da razão.

Observação 4.18 Calculando a série derivada em c, obtemos

$$
\frac{\partial^{\alpha}}{\partial x^{\alpha}} f(c)=\alpha ! a_{\alpha}
$$


O próximo teorema, que enunciamos sem demonstração, é uma versão da fórmula de Taylor.

Teorema 4.19 Seja $\mathrm{f}: \mathrm{U} \subset \mathbb{R}^{n} \longrightarrow \mathbb{C}$, onde $\mathrm{U}$ é aberto e convexo, $\mathrm{N}$ vezes diferenciável. Então dados $x, z \in \mathrm{U}$ existe um ponto $\xi$ no segmento que liga $x e$ $z$ tal que

$$
f(z)=\sum_{|\alpha| \leq N} \frac{1}{\alpha !} \frac{\partial^{\alpha}}{\partial x^{\alpha}} f(y)(z-y)^{\alpha}+\sum_{|\alpha|=N+1} \frac{1}{\alpha !} \frac{\partial^{\alpha}}{\partial x^{\alpha}} f(\xi)(z-y)^{\alpha}
$$

Lema 4.20 Se a e b são números reais satisfazendo $|\mathrm{a}|+|\mathrm{b}|<1$ então

1.

$$
\sum_{i=0}^{\infty} \sum_{j=0}^{\infty}\left(\begin{array}{c}
i+j \\
j
\end{array}\right) a^{i} b^{j}=\frac{1}{1-a-b}
$$

2.

$$
\sum_{\alpha \in \mathbb{N}^{m}} \sum_{\beta \in \mathbb{N}^{m}}\left(\begin{array}{c}
\alpha+\beta \\
\beta
\end{array}\right) a^{|\alpha|} b^{|\beta|}=\frac{1}{(1-a-b)^{m}}
$$

Demonstração. Para todo $m \in \mathbb{N}$, temos

$$
\sum_{k=0}^{m}\left(\begin{array}{c}
m \\
k
\end{array}\right) a^{k} b^{m-k}=(a+b)^{m}
$$

e portanto

$$
\sum_{m=0}^{\infty} \sum_{k=0}^{m}\left(\begin{array}{c}
m \\
k
\end{array}\right) a^{k} b^{m-k}=\frac{1}{1-a-b}
$$

que é um rearranjo da série em (1).

A igualdade em (2) segue de (1), e do fato de que

$$
\left(\begin{array}{c}
\alpha+\beta \\
\beta
\end{array}\right)=\prod_{j=1}^{n}\left(\begin{array}{c}
\alpha_{j}+\beta_{j} \\
\beta_{j}
\end{array}\right)
$$

Proposição 4.21 Seja

$$
\sum_{\alpha \in \mathbb{N}^{n}} a_{\alpha}(x-c)^{\alpha}
$$

uma série de potências e $\mathcal{C}$ seu dominio de convergência (que não se reduz a $\{\mathrm{c}\})$. Se $\mathrm{f}: \mathcal{C} \longrightarrow \mathbb{C}$ é definida por

$$
f(x)=\sum_{\alpha \in \mathbb{N}^{n}} a_{\alpha}(x-c)^{\alpha}
$$

então f é analítica real. 
DemonstraçÃo. Podemos assumir que $c=0$. Seja $x \in \mathcal{C}$. Por simplicidade de notação, vamos supor que $x_{j} \neq 0$ para todo $j$. Podemos escolher $R>0$ tal que

$$
(1+R) x \in \mathcal{C} .
$$

Então existe uma constante $C$ tal que $\left|a_{\alpha} \|(1+R) x\right|^{\alpha} \leq C$. Defina

$$
b_{\beta}=\frac{1}{\beta !} \frac{\partial^{\beta}}{\partial x^{\beta}} f(x)
$$

e observe que

$$
b_{\beta}=\sum_{\alpha \in \mathbb{N}^{n}} \frac{(\alpha+\beta)_{\beta}}{\beta !} a_{\alpha+\beta} x^{\alpha} .
$$

Escolha $0<\rho<R$. Considere $y \in \mathbb{R}^{m}$, com $\left|y_{j}-x_{j}\right| \leq \rho\left|x_{j}\right|$, para todo $j$. Com isso, podemos estimar

$$
\begin{gathered}
\sum_{\beta \in \mathbb{N}^{n}}\left|b_{\beta} \| y-x\right|^{\beta}=\sum_{\beta \in \mathbb{N}^{n}} \frac{1}{\beta !}\left|\frac{\partial^{|\beta|}}{\partial x^{\beta}} f(x)\right||y-x|^{\beta}= \\
=\sum_{\beta \in \mathbb{N}^{n}} \sum_{\alpha \in \mathbb{N}^{n}} \frac{(\alpha+\beta)_{\beta}}{\beta !}\left|a_{\alpha+\beta}\right||x|^{\alpha}|y-x|^{\beta} \leq \\
\leq C \sum_{\alpha, \beta \in \mathbb{N}^{n}} \frac{(\alpha+\beta)_{\beta}}{\beta !} \frac{\rho^{|\beta|}}{(1+R)^{|\alpha|+|\beta|}}=C\left(\frac{1+R}{R-\rho}\right)^{m} .
\end{gathered}
$$

Note que, para algum $\xi$ no segmento de reta de $x$ até $y$, temos

$$
\begin{gathered}
f(y)-\sum_{|\beta| \leq N} b_{\beta}(y-x)^{\beta}=\sum_{|\beta|=N+1} \frac{1}{\beta !} \frac{\partial^{\beta}}{\partial x^{\beta}} f(\xi)(z-y)^{\alpha}= \\
\sum_{|\beta|=N+1} \sum_{\alpha \in \mathbb{N}^{n}} \frac{(\alpha+\beta)_{\beta}}{\beta !} a_{\alpha+\beta} x^{\alpha}(y-x)^{\beta} .
\end{gathered}
$$

Assim, podemos estimar

$$
\begin{gathered}
\left|f(y)-\sum_{\beta \leq N} b_{\beta}(y-x)^{\beta}\right| \leq \sum_{|\beta|=N+1} \sum_{\alpha \in \mathbb{N}^{n}} \frac{(\alpha+\beta)_{\beta}}{\beta !}\left|a_{\alpha+\beta}\right||x|^{\alpha}|y-x|^{\beta} \leq \\
\leq C \sum_{|\beta|=N+1} \sum_{\alpha \in \mathbb{N}^{n}} \frac{(\alpha+\beta)_{\beta}}{\beta !} \frac{T^{|\beta|}}{(1+T)^{|\alpha+\beta|}},
\end{gathered}
$$

e observe que a última série converge para zero, quando $\mathrm{N}$ tende ao infinito. 
De modo a ficarmos de acordo com a notação utilizada em [B2], é necessário mudarmos a notação que se refere a funções periódicas. Note primeiro, que existe uma bijeção $\phi$ entre funções periódicas em $\mathbb{R}^{n}$, e funções definidas no toro $\mathbb{T}^{n}$. De fato, seja

$$
\begin{gathered}
A=\left\{f: \mathbb{R}^{n} \longrightarrow \mathbb{C} ; f \text { é } 2 \pi \text {-periódica }\right\}, \\
B=\left\{g: \mathbb{T}^{n} \longrightarrow \mathbb{C}\right\} .
\end{gathered}
$$

Se $f \in A$ então $g(\bar{x})=f(x)$, e basta definir $\phi(f)=g$, onde $g(\bar{x})=f(x)$. A partir de agora iremos escrever $\mathcal{D}^{\prime}\left(\mathbb{T}^{n}\right)$ ao invés de $\mathcal{P}_{2 \pi}^{\prime}\left(\mathbb{R}^{n}\right)$, e $C^{\infty}\left(\mathbb{T}^{n}\right)$ ao invés de $\mathcal{P}_{2 \pi}\left(\mathbb{R}^{n}\right)$.

O próximo teorema (veja [B1]) é relativamente simples, porém sustenta toda a demonstração de nosso objetivo principal nessa dissertação. Assim como obtivemos caracterizações de funções $C^{\infty}\left(\mathbb{T}^{n}\right)$ através de seus coeficientes de Fourier, o teorema abaixo identifica funções analíticas reais com coeficientes parciais que decaem uniformemente, e exponencialmente.

Teorema 4.22 Seja $f \in \mathcal{D}^{\prime}\left(\mathbb{T}^{p} \times \mathbb{T}^{q}\right)$ dada por sua série parcial de Fourier

$$
f=\sum_{\ell \in \mathbb{Z}^{q}} f_{\ell} e^{i \ell x} .
$$

Então $f \in \mathbb{C}^{\omega}\left(\mathbb{T}^{p} \times \mathbb{T}^{\mathrm{q}}\right)$ se e somente se $\mathrm{f}_{\ell} \in \mathbb{C}^{\omega}\left(\mathbb{T}^{\mathrm{p}}\right)$, para todo $\ell \in \mathbb{Z}^{\mathrm{q}}$ e existem $C, \varepsilon, \delta>0$, tais que

$$
\left|f_{\ell}(t+i s)\right| \leq C e^{-\delta|\ell|},
$$

para $\mathrm{t}+$ is $\in \mathbb{R}^{\mathrm{p}} \times[-\varepsilon, \varepsilon]^{\mathrm{p}} \subset \mathbb{C}^{\mathrm{p}}$.

DEMONSTRAÇÃo. $(\Longrightarrow)$ Suponha que $f \in \mathbb{C}^{\omega}\left(\mathbb{T}^{p} \times \mathbb{T}^{q}\right)$, então, pela proposição 4.16 , existem $\varepsilon, \delta>0$ tais que $f$ se estende a uma função analítica (como função complexa) no conjunto

$$
\left\{(t+i s, x+i y) ; t \in \mathbb{R}^{p}, s \in[-\varepsilon, \varepsilon]^{p}, x \in \mathbb{R}^{q}, y \in[-\delta, \delta]^{q}\right\},
$$

onde $t=\left(t_{1}, \ldots, t_{p}\right), s=\left(s_{1}, \ldots, s_{p}\right), x=\left(x_{1}, \ldots, x_{q}\right), y=\left(y_{1}, \ldots, y_{q}\right)$.

Como $f$ é de fato uma função integrável, se $\ell \in \mathbb{Z}^{\mathrm{q}}$ então

$$
f_{\ell}=\int_{[0,2 \pi]^{q}} f(t, x) e^{-i \ell x} d x
$$

e como a analiticidade do integrando é preservada pela integral, segue que

$$
f_{\ell}(t+i s)=\int_{[0,2 \pi]^{q}} f(t+i s, x) e^{-i \ell x} d x
$$

está bem definida, para todo $\ell \in \mathbb{Z}^{\mathrm{q}},(\mathrm{t}+\mathrm{is}) \in \mathbb{R}^{\mathrm{p}} \times[-\varepsilon, \varepsilon]^{\mathrm{p}}$. Além disto, $\mathrm{f}_{\ell} \in \mathbb{C}^{\omega}\left(\mathbb{T}^{\mathrm{p}}\right)$, $\ell \in \mathbb{Z}^{\mathrm{q}}$. 
Antes de mostrarmos que vale a desigualdade 4.23 , note que se $F: \mathbb{R} \times[-\eta, \eta] \longrightarrow$ $\mathbb{C}, \mathbb{R} \times[-\eta, \eta] \subset \mathbb{C}$, é uma função holomorfa tal que $F(x)=F(x+2 \pi)$, para todo $x \in \mathbb{R}$ então as funções holomorfas $\mathrm{F}(z), \mathrm{F}(z+2 \pi)$ coincidem num conjunto não enumerável e, portanto, são iguais em $\mathbb{R} \times[-\eta, \eta]$. Disso podemos concluir, se $\sigma= \pm 1$, e $[a, b]$ é o segmento de reta em $\mathbb{C}$ que liga $a, b$, então

$$
\int_{[\sigma i \eta, 0]} \mathrm{F}(z) \mathrm{d} z=\int_{\eta}^{0} \mathrm{~F}(\mathrm{ir}) \mathrm{dr}=\int_{\eta}^{0} \mathrm{~F}(2 \pi+i r) \mathrm{dr}=-\int_{[2 \pi, 2 \pi+\sigma i \eta]} \mathrm{F}(z) \mathrm{d} z .
$$

Para mostrar a desigualdade 4.23 , defina, para $j=1, \ldots, q$,

$$
\sigma\left(\ell_{j}\right)=\left\{\begin{array}{cc}
0 & \text { se } \ell_{j}=0 \\
\frac{\ell_{j}}{\left|\ell_{j}\right|} & \text { se } \ell_{j} \neq 0
\end{array}\right.
$$

onde $\ell=\left(\ell_{1}, \ldots, \ell_{\mathrm{q}}\right)$. Defina $\sigma(\ell)=\left(\sigma\left(\ell_{1}\right), \ldots, \sigma\left(\ell_{\mathrm{q}}\right)\right)$. Usando o teorema de Cauchy, o teorema de Fubini, e o discutido em 4.24, em cada iteração da integral, podemos escrever

$$
\begin{gathered}
\left.f_{\ell}(t+i s)=\int_{[0,2 \pi]^{q}} f(t+i s, x)\right) e^{i \ell x} d x= \\
=\int_{[0,2 \pi]^{q}} f_{\ell}(t+i s, x-i \sigma(\ell) \delta) e^{-i \ell(x-\sigma(\ell) i \delta)} d x= \\
=e^{-\delta \sigma \ell} \int_{[0,2 \pi]^{q}} f_{\ell}(t+i s, x-i \sigma(\ell) \delta) e^{-i \ell x} d x,
\end{gathered}
$$

portanto,

$$
\left|f_{\ell}(t+i s)\right|=e^{-\delta|\ell|}\left|\int_{[0,2 \pi] q} f_{\ell}(t+i s, x-i \sigma(\ell) \delta) e^{-i \ell x} d x\right| \leq e^{-\delta|\ell|} \sup A,
$$

onde

$$
A=\left\{|f(t+i s, x+i y)| ; t+i s \in \mathbb{R}^{p} \times[-\varepsilon, \varepsilon]^{p},(x+i y) \in \mathbb{R}^{q} \times[-\delta, \delta]^{q}\right\} .
$$

$(\Longleftarrow)$ Suponha que $f_{\ell} \in C^{\omega}\left(\mathbb{T}^{p}\right), \ell \in \mathbb{Z}^{q}$ e que existam $C, \varepsilon, \delta>0$ tais que vale 4.23. Se $s=0$, pelo teorema 3.19, página 55, concluímos que $f \in C^{\infty}\left(\mathbb{T}^{n}\right)$. Como $e^{-i \ell x} \in C^{\omega}\left(\mathbb{T}^{q}\right)$, pela proposição 4.16 existe $0<\delta_{1}<\delta$, tal que

$$
f(t+i s, x+i y)=\sum_{\ell \in \mathbb{Z}^{q}} f_{\ell}(t+i s) e^{i \ell(x+i y)}
$$

está bem definida em $D=\left(\mathbb{R}^{p} \times[-\varepsilon, \varepsilon]^{p}\right) \times\left(\mathbb{R}^{q} \times\left[-\delta_{1}, \delta_{1}\right]^{q}\right) \subset \mathbb{C}^{n}$. Resta mostrar que $f$ é holomorfa em $D$. Note que o termo geral da série acima satisfaz

$$
\left|f_{\ell}(t+i s) e^{i \ell(x+i y)}\right| \leq\left|f_{\ell}(t+i s)\right| e^{\ell|y|} \leq C e^{-\delta|\ell|} e^{|\ell| y \mid} \leq C e^{-\delta|\ell|} e^{\delta_{1}|\ell|}=C e^{-\left(\delta-\delta_{1}\right)|\ell|} .
$$


Pelo critério de Weierstrass concluímos que f é uma série de funções analíticas que converge uniformemente em cada subconjunto compacto de $\mathrm{D}$, e com isso obtemos que $f$ analítica em $D$ e, portanto, $f \in C^{\omega}\left(\mathbb{T}^{2}\right)$. 


\section{Capítulo 5}

\section{Expansões Assintóticas}

O objetivo deste capítulo é ilustrar um método que nos permite obter sob determinadas condições, expansões assintóticas para integrais dependentes de um parâmetro. Como consequência obtemos o método da fase estacionária no caso de funções analíticas reais.

Estamos interessados em estudar o comportamento assintótico de funções do tipo

$$
\mathrm{I}(\lambda)=\int_{\gamma} \mathrm{g}(z) \mathrm{e}^{\lambda \mathrm{f}(z)} \mathrm{d} z, \quad \lambda \longrightarrow \infty,
$$

onde $\gamma$ é um caminho no plano tal que $f$ e $g$ são holomorfas num domínio em $\mathbb{C}$ contendo $\gamma$.

O método consiste em deformar $\gamma$ (quando possível), segundo o teorema de Cauchy, de tal forma que o integrando assuma um formato mais calculável.

Quando $u=\operatorname{Re} f(z)$ possui um ponto de sela não degenerado, como no lema de Morse holomorfo 5.1, existe um caminho passando por $z_{0}$ que nos permite obter uma fórmula assintótica para $\mathrm{I}(\lambda)$. $\mathrm{O}$ caminho $\alpha$ em questão é chamado de caminho de maior descida, e é, num certo sentido, onde $\operatorname{Re} f(z)$ decresce mais rapidamente.

Para obtermos alguma informação a respeito de $\alpha$, suponha que $f(x+y i)=u(x, y)+i v(x, y), u=\operatorname{Re} f, v=\operatorname{Im} f$, é uma função holomorfa num aberto do plano complexo tal que $\nabla u(x, y) \neq 0$ em todo ponto do seu domínio. Em cada ponto $x+i y$, a direção de maior crescimento de $u$ é dada por $\nabla u(x, y)$, enquanto que a direção de maior decrescimento de $u$ é dada por $-\nabla u(x, y)$.

Assim, o caminho passando por $\left(x_{0}, y_{0}\right)$ que possui $-\nabla u(x, y)$ como vetor tangente em $(x, y)$ é o caminho de maior descida (decrescimento) em questão, ou seja, um caminho tal que todos seus vetores tangentes são direções de maior descida. Mais precisamente,

$$
\alpha=\left\{(x(t), y(t)) \in \mathbb{C} ; \quad\left(x^{\prime}(t), y^{\prime}(t)\right)=-\nabla u(x(t), y(t))\right\} .
$$

Note ainda que

$$
\alpha=\left\{(x(t), y(t)) \in \mathbb{C} ; \quad v(x(t), y(t))=v\left(x_{0}, y_{0}\right)\right\} .
$$


De fato, como valem as equações de Cauchy-Riemann para $u+i v$, temos

$$
\begin{gathered}
\frac{d v(x(t), y(t))}{d t}=v_{x}(x(t), y(t)) x^{\prime}(t)+v_{y}(x(t), y(t)) y^{\prime}(t)= \\
=-u_{y}(x(t), y(t)) x^{\prime}(t)+u_{x}(x(t), y(t)) y^{\prime}(t)= \\
=u_{y}(x(t), y(t)) u_{x}(x(t), y(t))-u_{x}(x(t), y(t)) u_{y}(x(t), y(t))=0 .
\end{gathered}
$$

No caso em que o gradiente de u se anula, podemos estender esta noção do seguinte modo.

Por direção de maior subida (crescimento de $u$ ) queremos dizer o seguinte: $e^{i \theta}$ é uma direção de maior subida em $x+i$, com relação a $u$, quando existe $\varepsilon>0$ tal que $u\left(x+i y+\tau e^{i \theta}\right)-u\left(x+i y+\tau e^{i \sigma}\right) \geq 0$, para todos $\tau \in[0, \varepsilon], \sigma \in[0,2 \pi]$. Para a direção de maior descida, devemos ter $u\left(x+i y+\tau e^{i \theta}\right)-u\left(x+i y+\tau e^{i \sigma}\right) \leq 0$, para todos $\tau \in[0, \varepsilon], \sigma \in[0,2 \pi]$.

Note que quando o gradiente da $u$ se anula em algum ponto, pode ocorrer mais de uma direção de maior crescimento ou decrescimento. Por exemplo, considere $f(z)=z^{2}$, ou seja, $f(x, y)=u(x, y)+i v(x, y)=x^{2}-y^{2}+i 2 x y$. Em $(0,0)$ as direções de maior descida com relação a u são dadas pelos vetores $(0,1)$ e $(0,-1)$. De fato, temos

$$
u\left(\tau e^{i \pi / 2}\right)-u\left(\tau e^{i \sigma}\right)=-\tau^{2}\left(1+\cos ^{2} \sigma-\sin ^{2} \sigma\right)=-\tau^{2}(1+\cos (2 \sigma)) \leq 0
$$

e também

$$
u\left(e^{i 3 \pi / 2}\right)-u\left(\tau e^{i \sigma}\right)=-\tau^{2}\left(1+\cos ^{2} \sigma-\sin ^{2} \sigma\right)=-\tau^{2}(1+\cos (2 \sigma)) \leq 0 .
$$

Note agora que os caminhos $\alpha_{1}(y)=(0, y), y \geq 0$ e $\alpha_{2}(y)=(0, y), y \leq 0$, são os caminhos de maior descida passando pelo ponto de sela $(0,0)$, pois cada um de seus pontos tem como vetor tangente uma direção de maior descida (para os outros pontos diferentes de $(0,0)$, basta usar o critério do gradiente).

No que diz respeito ao cálculo de $\mathrm{I}(\lambda)$, o lema de Morse (versão holomorfa) garante que basta o conhecimento do caso $f(z)=z^{2}$ para obtermos os demais.

Lema 5.1 (Lema de Morse versão holomorfa) Seja $f: \mathrm{B}(0, \delta) \longrightarrow \mathbb{C}, \mathrm{B}(0, \delta) \subset \mathbb{C}$ uma função holomorfa tal que $\mathrm{f}(0)=0, \mathrm{f}^{\prime}(0)=0$ e $\mathrm{f}^{\prime \prime}(0) \neq 0$.

Então existem uma bola aberta B centrada na origem e uma função holomorfa $\phi: \mathrm{B} \longrightarrow \phi(\mathrm{B})$ invertivel com inversa também holomorfa tal que $f\left(\phi^{-1}(w)\right)=w^{2}$, para todo $w \in \phi(B)$.

Demonstração. Podemos fazer

$$
f(z)=\sum_{n=2}^{\infty} \frac{f^{(n)}(0)}{n !} z^{n}=z^{2} \sum_{\ell=0}^{\infty} \frac{f^{(\ell+2)}(0)}{(\ell+2) !} z^{\ell}=z^{2} g(z)
$$


onde

$$
g(z)=\sum_{\ell=0}^{\infty} \frac{f^{(\ell+2)}(0)}{(\ell+2) !} z^{\ell}
$$

Note que $g(0)=f^{\prime \prime}(0) / 2 \neq 0$ e, portanto, existe $B(0, \delta)$, onde $g$ admite um logaritmo. Assim, existe $h: B(0, \delta) \longrightarrow \mathbb{C}$ holomorfa tal que $g(z)=h^{2}(z)$. Portanto,

$$
f(z)=z^{2} g(z)=z^{2} h^{2}(z)=(z h(z))^{2}, \quad z \in B(0, \delta) .
$$

Defina $\phi(z)=z h(z), z \in B(0, \delta)$. Note que $\phi^{\prime}(0)=h(0) \neq 0$ e, pelo teorema da função inversa, existe $\delta^{\prime}>0$ tal que $\mathrm{B} \doteq \mathrm{B}\left(0, \delta^{\prime}\right) \subset \mathrm{B}(0, \delta)$ e $\phi: \mathrm{B} \longrightarrow \phi(\mathrm{B})$ é invertível com inversa holomorfa. Assim, se $w \in \phi(B)$, isto é, $w=\phi(z), z \in B$, então

$$
f\left(\phi^{-1}(w)\right)=f(z)=(z h(z))^{2}=\phi^{2}(z)=w^{2} .
$$

Corolário 5.2 Seja $f: D \longrightarrow \mathbb{C}$ holomorfa tal que $f^{\prime}(0)=0, f^{\prime \prime}(0) \neq 0$, onde D é um aberto contendo 0 . Então existem $\mathrm{A} \doteq \mathrm{B}(0, \rho)$ e $\psi: A \longrightarrow \psi(\mathrm{A})$ holomorfa com inversa holomorfa tais que, se $\alpha(s)=\psi($ is $), s \in \mathbb{R},|s|<\rho$, então $\alpha(s)$ é uma curva que satisfaz $\mathrm{f}(\alpha(\mathrm{s}))=-\mathrm{s}^{2}+\mathrm{f}(0)$.

Demonstração. Sejam $\phi$ e B como no lema de Morse aplicado à função $f(z)-f(0)$. Note que $\phi(0)=0$. Seja $A \subset \phi(B)$ um a bola aberta centrada na origem. Coloque $\psi=\phi^{-1}: A \rightarrow \psi(A)$. Temos

$$
f(\psi(t+i s))=f(0)+t^{2}-s^{2}+i 2 t s
$$

e, colocando $t=0$, obtemos

$$
f(\alpha(s))=-s^{2}+f(0),
$$

para todo $s \in \mathbb{R},|s|<\rho$.

Proposição 5.3 Sejam $\mathrm{f}$ e $\alpha$ como acima. Então $\alpha^{\prime}(0)=\sqrt{\frac{2}{-f^{\prime \prime}(0)}}$, para algum ramo apropriado da raiz quadrada.

Demonstração. Temos que, numa vizinhança conveniente,

$$
\begin{gathered}
f(z)=f(0)+\frac{f^{\prime \prime}(0)}{2} z^{2}+\sum_{n=3}^{\infty} \frac{f^{(n)}(0)}{n !} z^{n} \\
f(\alpha(s))=f(0)-s^{2} .
\end{gathered}
$$


Temos

$$
-s^{2}=\frac{f^{\prime \prime}(0)}{2} \alpha^{2}(s)+R(s), \quad R(s) \doteq \sum_{n=3}^{\infty} \frac{f^{(n)}(0)}{n !} \alpha^{n}(s),
$$

ou seja,

$$
\alpha^{2}(s)=\frac{2}{f^{\prime \prime}(0)}\left(-R(s)-s^{2}\right)
$$

onde $R(s)=o\left(s^{2}\right), R^{\prime}(s)=o(s), s \longrightarrow 0$. Derivando 5.4

$$
2 \alpha(s) \alpha^{\prime}(s)=-\frac{2}{f^{\prime \prime}(0)}\left(R^{\prime}(s)+2 s\right)
$$

obtemos,

$$
\lim _{s \rightarrow 0} 2 \frac{\alpha(s)}{s} \alpha^{\prime}(s)=\lim _{s \rightarrow 0}-\frac{2}{f^{\prime \prime}(0)}\left(\frac{R^{\prime}(s)}{s}+2\right)
$$

isto é,

$$
\left(\alpha^{\prime}(0)\right)^{2}=-\frac{2}{f^{\prime \prime}(0)}
$$

Nosso objetivo agora é mostrar que o caminho $\alpha$ da proposição anterior, é o que simplifica o cálculo de $\mathrm{I}(\lambda)=\int_{\gamma} g(z) e^{\lambda f(z)} \mathrm{d} z$. Apesar desse caminho ser definido localmente, já é suficiente, uma vez que a integral $\mathrm{I}(\lambda)$ é concentrada em 0 , no seguinte sentido.

Seja $u=\operatorname{Ref}$. A curva $\{(x, y) \in D \subset \mathbb{C} ; \mathfrak{u}(x, y)=0\}$ divide $D$ em regiões $D_{+}=\{(x, y) \in D ; u(x, y)>0\}$ e $D_{-}=\{(x, y) \in D ; u(x, y)<0\}$. Sejam $b>a>0$ e $\beta:[-b, b] \longrightarrow D$ diferenciável tal que $\beta \subset D_{-} \cup\{0\}$ e, além disto, $\beta_{[-a, a]}=\alpha$. Portanto, podemos fazer

$$
\begin{gathered}
\int_{\beta} g(z) e^{\lambda f(z)} d z=\int_{-b}^{b} g(\beta(s)) e^{\lambda f(\beta(s))} \beta^{\prime}(s) d s= \\
=\int_{-b}^{-a} g(\beta(s)) e^{\lambda f(\beta(s))} \beta^{\prime}(s) d s+\int_{-a}^{a} g(\beta(s)) e^{\lambda f(\beta(s))} \beta^{\prime}(s) d s+ \\
+\int_{a}^{b} g(\beta(s)) e^{\lambda f(\beta(s))} \beta^{\prime}(s) d s .
\end{gathered}
$$

Podemos supor que $u(\beta(s))$ assume um máximo negativo em $[a, b]$, digamos, $-\mu$. Disto segue que

$$
\begin{gathered}
\left|\int_{a}^{b} g(\beta(s)) e^{\lambda f(\beta(s))} \beta^{\prime}(s) d s\right| \leq \int_{a}^{b}\left|g(\beta(s)) \beta^{\prime}(s)\right| e^{\lambda u(\beta(s))} d s \leq \\
\leq K e^{-\lambda \mu} \int_{a}^{b} d s
\end{gathered}
$$


para algum $K>0$. Um resultado análogo vale para

$$
\int_{-b}^{-a} g(\beta(s)) e^{\lambda f(\beta(s))} \beta^{\prime}(s) d s .
$$

Portanto, a integral em $\beta$ se reduz a

$$
\int_{\beta} g(z) e^{\lambda f(z)} d z=\int_{\alpha} g(z) e^{\lambda f(z)} d z+O\left(e^{-\lambda \eta}\right)
$$

para algum $\eta>0$. Portanto, se uma das integrais acima não decai exponencialmente quando $\lambda$ cresce indefinidamente, o mesmo acontece com a outra.

A seguir, vamos obter uma expressão assintótica para $\int_{\alpha} g(z) e^{\lambda f(z)} d z$. Temos que

$$
\begin{gathered}
\int_{\alpha} g(z) e^{\lambda f(z)} d z=\int_{-a}^{a} g(\alpha(s)) e^{\lambda f(\alpha(s))} \alpha^{\prime}(s) d s= \\
=\int_{-a}^{a} g(\alpha(s)) \alpha^{\prime}(s) e^{-\lambda\left(-f(0)+s^{2}\right)} d s=e^{\lambda f(0)} \int_{-a}^{a} G(s) e^{-\lambda s^{2}} d s
\end{gathered}
$$

onde $G(s)=g(\alpha(s)) \alpha^{\prime}(s)$. Note que $G(s)=G(0)+s H(s), H \in C^{1}([-a, a])$. Com isso obtemos

$$
\begin{gathered}
\int_{-a}^{a} G(s) e^{-\lambda s^{2}} d s=\int_{-a}^{a} G(0) e^{-\lambda s^{2}} d s+\int_{-a}^{a} H(s) e^{-\lambda s^{2}} s d s= \\
=G(0) \int_{-a}^{a} e^{-\lambda s^{2}} d s+(2 \lambda)^{-1} \int_{-a}^{a} H^{\prime}(s) e^{-\lambda s^{2}} d s-\left.(2 \lambda)^{-1} H(s) e^{-\lambda s^{2}}\right|_{-a} ^{a} .
\end{gathered}
$$

Como $\mathrm{H}^{\prime}(\mathrm{s})$ é limitada em $[-\mathrm{a}, \mathrm{a}]$, valem

$$
\begin{gathered}
\frac{1}{2 \lambda} \int_{-a}^{a} H^{\prime}(s) e^{-\lambda s^{2}} d s=O\left(\lambda^{-1}\right) \int_{-a}^{a} e^{-\lambda s^{2}} d s \\
\left.(2 \lambda)^{-1} H(s) e^{-\lambda s^{2}}\right|_{-a} ^{a}=O\left(e^{-a^{2} \lambda}\right) .
\end{gathered}
$$

Assim, concluímos que

$$
\begin{gathered}
\int_{\alpha} g(z) e^{\lambda f(z)} d z=e^{\lambda f(0)} \int_{-a}^{a} G(s) e^{-\lambda s^{2}} d s= \\
=e^{\lambda f(0)} G(0) \int_{-a}^{a} e^{-\lambda s^{2}} d s\left(1+O\left(\lambda^{-1}\right)\right)+e^{\lambda f(0)} O\left(e^{-a^{2} \lambda}\right)= \\
=e^{\lambda f(0)} G(0) \sqrt{\frac{\pi}{\lambda}}\left(1+O\left(\lambda^{-1}\right)\right)+O\left(e^{-c \lambda}\right),
\end{gathered}
$$

para algum c $>0$. Utilizando a proposição 5.3, obtemos a seguinte fórmula assintótica

$$
\int_{\alpha} g(z) e^{\lambda f(z)} d z=\frac{g(0)}{\sqrt{-f^{\prime \prime}(0)}} e^{\lambda f(0)} \sqrt{\frac{2 \pi}{\lambda}}\left(1+O\left(\lambda^{-1}\right)\right)+O\left(e^{-c \lambda}\right) .
$$


Proposição 5.6 (Método da Fase Estacionária) Considere a integral

$$
I(\lambda)=\int_{-\delta}^{\delta} g(t) e^{i \lambda f(t)} d t
$$

onde $\mathrm{f}, \mathrm{g}$ são funções analíticas reais de uma variável tais que $f$ é a valores reais, $f(0)=0, f^{\prime}(0)=0$ e $f^{\prime \prime}(0) \neq 0$. Então, para $\delta>0$ suficientemente pequeno,

$$
\mathrm{I}(\lambda)=\frac{\mathrm{g}(0)}{\sqrt{-\mathrm{f}^{\prime \prime}(0)}} \sqrt{\frac{2 \pi}{\lambda}}\left(1+\mathrm{O}\left(\lambda^{-1}\right)\right)+\mathrm{O}\left(\mathrm{e}^{-\mathrm{c} \lambda}\right)
$$

onde $\mathrm{c}>0, \lambda \longrightarrow \infty$.

Demonstração. Como $f, g$ são analíticas reais, existe $\rho>0$, tal que $f$ e $g$ se estendem a funções holomorfas em alguma bola $B(0, \rho)$. Continuaremos usando a mesma notação para estas extensões. Estamos então nas condições do método da maior descida, para a função if.

Temos que $f=u+i v$ em $B(0, \rho)$. Seja

$$
\mathrm{D}=\{\operatorname{Re}(\text { if }) \leq 0\}=\{z \in \mathrm{B}(0, \rho) ;-v(z) \leq 0\}
$$

Note que se $\phi$ é a função garantida pelo lema de Morse, cuja inversa está definida numa bola $\mathrm{B}\left(0, \rho_{1}\right)$, onde $\phi^{-1}\left(\mathrm{~B}\left(0, \rho_{1}\right)\right) \subset \mathrm{B}(0, \rho)$, então if $\left(\phi^{-1}(z)\right)=z^{2}, z \in \mathrm{B}\left(0, \rho_{1}\right)$.

Considere o conjunto $A=\left\{(t, s) \in B\left(0, \rho_{1}\right) ;|t| \leq|s|\right\}$, isto é,

$$
A=\left\{(t, s) \in B\left(0, \rho_{1}\right) ; \operatorname{Re}\left(i f\left(\phi^{-1}(t, s)\right)\right) \leq 0\right\} .
$$

Note que $\phi^{-1}(A)=D \cap \phi^{-1}\left(B\left(0, \rho_{1}\right)\right)$.

Como $\phi$ é contínua, existe $\delta>0$ tal que $\phi(-\delta), \phi(\delta) \subset \mathrm{B}\left(0, \rho_{1}\right)$. Note também que $-\delta, \delta \in D$, pois $f$ é a valores reais. Portanto $-\delta, \delta \in \phi^{-1}(A)$.

Deste modo, existe um caminho $\beta$ contido em $A$ ligando $\phi(-\delta)$ a $\phi(\delta)$ e passando pela origem. Assim, o caminho $\gamma=\phi^{-1} \circ \beta$ está contido em $\mathrm{D}$, liga $-\delta$ a $\delta$ e passa pela origem e pode ser usado o método da maior descida. 


\section{Capítulo 6}

\section{Hipoeliticidade Analítica Global de Campos Vetoriais no Toro $\mathbb{T}^{2}$}

\subsection{Hipoeliticidade Analítica Global do Operador $\mathrm{L}=\partial_{\mathrm{t}}+\mathrm{c}(\mathrm{t}) \partial_{x}$ em $\mathcal{D}^{\prime}\left(\mathbb{T}^{2}\right)$}

Definição 6.1 Dizemos que um operador $\mathrm{L}: \mathcal{D}^{\prime}\left(\mathbb{T}^{2}\right) \longrightarrow \mathcal{D}^{\prime}\left(\mathbb{T}^{2}\right)$ é globalmente analítico hipoelítico (GAH) quando vale

$$
\left(\mathrm{Lu}=\mathrm{f}, \quad \mathrm{u} \in \mathcal{D}^{\prime}\left(\mathbb{T}^{2}\right), \quad f \in \mathrm{C}^{\omega}\left(\mathbb{T}^{2}\right)\right) \Longrightarrow u \in \mathrm{C}^{\omega}\left(\mathbb{T}^{2}\right)
$$

Estamos agora frente ao objetivo principal desta dissertação, que é responder à pergunta:

Quais são os operadores em $\mathcal{D}^{\prime}\left(\mathbb{T}^{2}\right)$ da forma $L=\partial_{t}+c(t) \partial_{x}, c \in C^{\omega}\left(\mathbb{S}^{1}\right)$, que são globalmente analítico hipoelíticos?

Tal pergunta foi respondida de maneira completa por Bergamasco em [B2]. Faremos aqui neste capítulo um estudo detalhado da demonstração apresentada em [B2], e forneceremos também uma prova alternativa, baseada em [BNZ], para a construção de uma solução singular de L. Por uma solução singular de L queremos dizer um elemento $u \in \mathcal{D}^{\prime}\left(\mathbb{T}^{2}\right) \backslash C^{\omega}\left(\mathbb{T}^{2}\right)$ tal que $L u \in C^{\omega}\left(\mathbb{T}^{2}\right)$. Para tal, precisamos antes da definição seguinte.

Definição 6.2 Dizemos que o número irracional a é exponencial Liouville, se existe $\varepsilon>0$ tal que a desigualdade $\left|a-\frac{p}{q}\right| \leq e^{-\varepsilon q}$ possui infinitas soluções racionais $\frac{\mathrm{p}}{\mathrm{q}}, \mathrm{p}, \mathrm{q} \in \mathbb{Z}, \mathrm{q} \geq 1$.

Com isso, podemos enunciar a resposta encontrada por Bergamasco, cuja demonstração será obtida no decorrer desse capítulo. 
Teorema 6.3 (Bergamasco) Seja $\mathrm{L}: \mathcal{D}^{\prime}\left(\mathbb{T}^{2}\right) \longrightarrow \mathcal{D}^{\prime}\left(\mathbb{T}^{2}\right)$ dado por $\mathrm{L}=\partial_{\mathrm{t}}+\mathrm{c}(\mathrm{t}) \partial_{\mathrm{x}}$, onde $\mathrm{c} \in \mathrm{C}^{\omega}\left(\mathbb{S}^{1}\right), \mathrm{c}(\mathrm{t})=\mathrm{a}(\mathrm{t})+\mathrm{ib}(\mathrm{t})$, $\mathrm{a}$ e $\mathrm{b}$ a valores reais. L é globalmente analítico hipoelítico (GAH) se e somente se

1. b não muda de sinal;

2. se b é identicamente nula, então o número real

$$
a_{0}=(2 \pi)^{-1} \int_{0}^{2 \pi} a(t) d t
$$

é irracional e não é exponencial Liouville.

\subsection{Resultados Preliminares}

Nesta seção desenvolvemos algumas ferramentas que se mostraram necessárias à demonstração do teorema 6.3.

\subsubsection{Solução das equações $L u=0$ e $L u=f$}

No que segue veremos que o lema 6.4 impõe uma condição sobre $c(t)$ para que tenhamos soluções de $\mathrm{Lu}=0$, enquanto que o lema 6.8 nos dá os coeficientes parciais de Fourier das soluções $u$ de $L u=f \in C^{\omega}\left(\mathbb{T}^{2}\right)$.

Seja

$$
\begin{gathered}
c_{0}=(2 \pi)^{-1} \int_{0}^{2 \pi} c(t) d t=(2 \pi)^{-1}\left(\int_{0}^{2 \pi} a(t) d t+i \int_{0}^{2 \pi} b(t) d t\right)= \\
=a_{0}+i b_{0}, \quad a_{0}, b_{0} \in \mathbb{R} .
\end{gathered}
$$

Lema 6.4 A distribuição u é solução da equação $\mathrm{Lu}=0 \mathrm{em} \mathcal{D}^{\prime}\left(\mathbb{T}^{2}\right)$ se e somente se vale uma das sentenças abaixo

1. $\mathrm{c}_{0} \notin \mathbb{Q}$ e u é constante.

2. $\mathrm{c}_{0}=\mathrm{p} / \mathrm{q}$ (forma irredutivel), $\mathrm{p} \in \mathbb{Z}, \mathrm{q} \in \mathbb{N}$ e u é limite em $\mathcal{D}^{\prime}\left(\mathbb{T}^{2}\right)$ de sequências da forma $\sum_{k \in F} \alpha_{k} v_{k}(t, x), \operatorname{com} \nu_{k}(x, t)=e^{i k q\left(x-\int_{0}^{t} c\right)}, \alpha_{k} \in \mathbb{C} e$ $\mathrm{F} \subset \mathbb{Z}$, finito.

DemonstraçÃo. Se uma das condições acima é válida, é imediato que u é solução da homogênea $(\mathrm{Lu}=0)$.

Suponha agora que u seja solução da homogênea. Tomando a série parcial de Fourier com relação a $x, u=\sum_{n \in \mathbb{Z}} u_{n} e^{i n x}, u_{n} \in \mathcal{D}^{\prime}\left(\mathbb{T}_{t}\right)$, segue da unicidade dos coeficientes parciais, que $\mathrm{Lu}=0$ se e somente se

$$
\dot{u}_{n}+i c n u_{n}=0 \text {. }
$$


As soluções da equação acima com $n \neq 0$ são da forma

$$
u_{n}(t)=\alpha_{n} e^{-\int_{0}^{t} i n c(s) d s}, \quad \alpha_{n} \in \mathbb{C}, \quad t \in \mathbb{R} .
$$

Portanto, $u_{n}$ é, de fato, uma função analítica real. Assim, as soluções formais de $\mathrm{Lu}=0$ são

$$
u(t, x)=\sum_{n \in \mathbb{Z}} u_{n}(0) e^{-\int_{0}^{t} i n c(s) d s} e^{i n x} .
$$

Note que para $n=0$, a única solução periódica de 6.5 é $u_{0}$ igual a constante. Vejamos quais as condições que devemos ter sobre as médias $a_{0}$ e $b_{0}$, no caso em que $u_{n}$ não é identicamente zero. Como $u_{n}(t)$ é $2 \pi$-periódica, para todo $n \in \mathbb{Z}$ e não identicamente zero, temos

$$
\begin{aligned}
& u_{n}(t)=u_{n}(t+2 \pi) \Longleftrightarrow \\
& e^{-\int_{0}^{t} \operatorname{inc}(s) \mathrm{d} s}=e^{-\int_{0}^{\mathrm{t}} \operatorname{inc}(s) \mathrm{d} s} e^{-\int_{\mathrm{t}}^{\mathrm{t}+2 \pi} \operatorname{inc}(s) \mathrm{d} s} \Longleftrightarrow \\
& e^{-\int_{0}^{2 \pi} \operatorname{inc}(s) \mathrm{d} s}=1 \Longleftrightarrow e^{-\int_{0}^{2 \pi} i n a(s) d s} e^{\int_{0}^{2 \pi} n b(s) d s}=1 \Longleftrightarrow \\
& 2 \pi n b_{0}=\int_{0}^{2 \pi} n b(s) d s=0 \quad \text { e } \quad \int_{0}^{2 \pi} n a(s) d s=2 \pi a_{0} n \in 2 \pi \mathbb{Z},
\end{aligned}
$$

para todo $n \in \mathbb{Z}$. Se $n \neq 0$ e $b_{0}=0$ então $a_{0}=r(n) / n$, com $r(n) \in \mathbb{Z}$, e portanto, $c_{0}=a_{0} \in \mathbb{Q}$. Coloque $c_{0}=p / q, p$ e q primos entre si. Como devemos ter $r(n) / n=$ $p / q$, vemos que $n p / q \in \mathbb{Z} e$, pela escolha de $p$ e $q$, isto só é possível se $n$ for múltiplo de $q$, digamos, $n=k q$. Assim, neste caso, por 6.6, obtemos que as soluções de $L u=0$ são da forma

$$
\sum_{k \in \mathbb{Z}} \alpha_{k} e^{i k q\left(x-\int_{0}^{t} c(s) d s\right)} .
$$

Assim, obtemos 2.

Agora, se $b_{0} \neq 0$ e $n \neq 0$, só podemos ter $u_{n}$ identicamente nula, pois caso contrário deveríamos ter $n b_{0}=0$. Assim, $c_{0}$ tem parte imaginária não nula, e portanto $u$ é constante. Obtemos desta forma 1 .

Lema 6.8 Suponha que $c_{0} \notin \mathbb{Q}$. Se $\mathrm{Lu}=\mathrm{f} \in \mathrm{C}^{\omega}\left(\mathbb{T}^{2}\right)$ possui solução, com as séries parciais de Fourier de $u$ e f dadas por $u=\sum_{n \in \mathbb{Z}} u_{n} e^{i n x}, u_{n} \in \mathcal{D}^{\prime}\left(\mathbb{T}_{t}\right) e$ $\mathrm{f}(\mathrm{t}, \mathrm{x})=\sum_{\mathrm{n} \in \mathbb{Z}} \mathrm{f}_{\mathrm{n}}(\mathrm{t}) e^{\mathrm{in} x}$. Então

$$
\int_{0}^{2 \pi} f_{0}(t) d t=0 .
$$

Além disto, os coeficientes parciais de u são dados por

$$
u_{0}(t)=\int_{0}^{t} f_{0}(s) d s+\text { constante. }
$$




$$
\begin{gathered}
u_{n}(t)=\left(1-e^{-i 2 \pi n c_{0}}\right)^{-1} \int_{0}^{2 \pi} e^{-i n \int_{t-s}^{t} c(r) d r} f_{n}(t-s) d s, n \in \mathbb{Z} \backslash\{0\} \\
u_{n}(t)=\left(e^{i 2 \pi n c_{0}}-1\right)^{-1} \int_{0}^{2 \pi} e^{i n \int_{t}^{t+s} c(r) d r} f_{n}(t+s) d s, n \in \mathbb{Z} \backslash\{0\} .
\end{gathered}
$$

DemonstraçÃo. Tomando-se as séries parciais de Fourier dos dois lados da equação $\mathrm{Lu}=\mathrm{f}$, obtemos que para todo $\mathrm{n} \in \mathbb{Z}$, devemos ter

$$
\dot{u}_{n}+i n c u_{n}=f_{n} .
$$

Como no caso das soluções da homogênea, segue aqui também que $u_{n} \in C^{\omega}(\mathbb{T})$, pois $f_{n} \in C^{\omega}(\mathbb{T})$.

Quando $n=0$, a equação 6.12 fica $\dot{u}_{0}=f_{0} e$, portanto, $u_{0}(2 \pi)-u_{0}(0)=$ $\int_{0}^{2 \pi} f_{0}(t) d t=0$, pois $u_{0}$ é $2 \pi$-periódica.

Ainda para $n=0$, integrando-se com relação a $t$ dos dois lados da equação, temos que $\mathrm{u}_{0}(\mathrm{t})=\int_{0}^{\mathrm{t}} \mathrm{f}_{0}(\mathrm{~s}) \mathrm{ds}+$ constante.

Para $n \neq 0$, a solução da EDO de primeira ordem em questão é dada pela igualdade

$$
u_{n}(t) e^{i n \int_{0}^{t} c(r) d r}-u_{n}(0)=\int_{0}^{t} e^{i n \int_{0}^{y} c(r) d r} f_{n}(y) d y .
$$

Como $u_{n}$ é $2 \pi$-periódica, devemos ter $u_{n}(t)=u_{n}(t-2 \pi)=u_{n}(t+2 \pi)$, o que nos permite fazer

$$
u_{n}(t) e^{i n \int_{0}^{t-2 \pi} c(r) d r}-u_{n}(0)=\int_{0}^{t-2 \pi} e^{i n \int_{0}^{y} c(r) d r} f_{n}(y) d y .
$$

Subtraindo-se a equação 6.14 de 6.13 , temos

$$
\begin{gathered}
u_{n}(t) e^{i n \int_{0}^{t} c(r) d r}\left(1-e^{-i n \int_{0}^{2 \pi} c(r) d r}\right)=-\int_{t}^{t-2 \pi} e^{i n \int_{0}^{y} c(r) d r} f_{n}(y) d y \Longrightarrow \\
\Longrightarrow u_{n}(t)\left(1-e^{-i n \int_{0}^{2 \pi} c(r) d r}\right)=\int_{t-2 \pi}^{t} e^{i n \int_{t}^{y} c(r) d r} f_{n}(y) d y .
\end{gathered}
$$

Fazendo-se a mudança $y=t-s$ no lado direito da igualdade anterior, concluímos (2), ou seja

$$
u_{n}(t)=\left(1-e^{-i 2 \pi n c_{0}}\right)^{-1} \int_{0}^{2 \pi} e^{-i n \int_{t-s}^{t} c(r) d r} f_{n}(t-s) d s .
$$

Partindo agora da igualdade

$$
u_{n}(t) e^{i n \int_{0}^{t+2 \pi} c(r) d r}-u_{n}(0)=\int_{0}^{t+2 \pi} e^{i n \int_{0}^{y} c(r) d r} d y,
$$


obtemos

$$
u_{n}(t)\left(e^{-i n \int_{0}^{2 \pi} c(r) d r}-1\right)=\int_{t}^{t+2 \pi} e^{i n \int_{t}^{y} c(r) d r} f_{n}(y) d y .
$$

Fazendo agora a mudança de variáveis $y=t+s$, chegamos em (3),

$$
u_{n}(t)=\left(e^{i 2 \pi n c_{0}}-1\right)^{-1} \int_{0}^{2 \pi} e^{i n \int_{t}^{t+s} c(r) d r} f_{n}(t+s) d s
$$

\subsubsection{Automorfismos e Equivalências}

Proposição 6.15 Sejam $a \in C^{\omega}(\mathbb{T} ; \mathbb{R})$ e $a_{0}=(2 \pi)^{-1} \int_{0}^{2 \pi} a(t) d t$. Seja $S: \mathcal{D}^{\prime}\left(\mathbb{T}^{2}\right) \rightarrow$ $\mathcal{D}^{\prime}\left(\mathbb{T}^{2}\right)$ definido por $\mathrm{Su}=v$ onde

$$
v=\sum_{n \in \mathbb{Z}} v_{n}(t) e^{i n x}
$$

e seus coeficientes parciais de Fourier são dados através dos coeficientes parciais de Fourier de

$$
u=\sum_{n \in \mathbb{Z}} u_{n}(t) e^{i n x}
$$

por

$$
v_{n}(t)=u_{n}(t) e^{i n\left[\int_{0}^{t} a(r) d r-a_{0} t\right]}, \quad n \in \mathbb{Z} .
$$

Então S é uma bijeção e $\mathrm{S}\left(\mathrm{C}^{\omega}\left(\mathbb{T}^{2}\right)\right)=\mathrm{C}^{\omega}\left(\mathbb{T}^{2}\right)$.

DEMONSTRAÇÃO.

$S$ é injetora, pois se $u=\sum_{n \in \mathbb{Z}} u_{n}(t) e^{i n x}$ e $w=\sum_{n \in \mathbb{Z}} w_{n}(t) e^{i n x}$ são tais que $S(u)=S(w)$, pela unicidade da série parcial de Fourier, temos que $S(u)=S(w)$ implica que

$$
\sum_{n \in \mathbb{Z}} u_{n}(t) e^{i n\left[\int_{0}^{t} a(r) d r-a_{0} t\right]} e^{i n x}=\sum_{n \in \mathbb{Z}} w_{n}(t) e^{i n\left[\int_{0}^{t} a(r) d r-a_{0} t\right]} e^{i n x}
$$

que por sua vez implica em $u_{n}(t)=w_{n}(t), n \in \mathbb{Z}, t \in[0,2 \pi]$, o que nos dá $u=w$.

$S$ é sobrejetora, pois dado $\sum_{n \in \mathbb{Z}} v_{n}(t) e^{i n x}=v \in \mathcal{D}^{\prime}\left(\mathbb{T}^{2}\right)$, basta tomar $u=$ $\sum_{n \in \mathbb{Z}} u_{n}(t) e^{i n x}, \quad \operatorname{com} u_{n}=v_{n}(t) e^{-i n\left[\int_{0}^{t} a(r) d r-a_{0} t\right]}, \quad$ para obtermos $\mathrm{Su}=v$. Note que $u \in \mathcal{D}^{\prime}\left(\mathbb{T}^{2}\right)$, pois $\left|u_{n}\right|=\left|v_{n}\right|$.

A fim de verificarmos $S\left(C^{\omega}\left(\mathbb{T}^{2}\right)\right)=C^{\omega}\left(\mathbb{T}^{2}\right)$, considere $u \in C^{\omega}\left(\mathbb{T}^{2}\right)$. Pela caracterização dos coeficientes de Fourier de uma função analítica real, $u_{n} \in C^{\omega}(\mathbb{T})$, para todo $n \in \mathbb{Z}$ e existem constantes $E, F, \delta>0$, tais que $\left|u_{n}(t+s i)\right| \leq$ $E e^{-|n| F}$, para todo $n \in \mathbb{Z}, t+s i \in[0,2 \pi] \times[-\delta, \delta]$.

Existe $0<v^{\prime}<\delta$ tal que a, $\operatorname{Re}\left(i \int_{0}^{t+i s} a(z) d z-i a_{0}(t+i s)\right)$ estão definidas em $[0,2 \pi] \times\left[-v^{\prime}, v^{\prime}\right]$. A função de $(t, s), \operatorname{Re}\left(i \int_{0}^{t+i s} a(z) d z-i a_{0}(t+i s)\right)$ é contínua 
em $[0,2 \pi] \times\left[-v^{\prime}, v^{\prime}\right]$, portanto, uniformemente contínua. Além do mais, vale $0 \mathrm{em}$ $[0,2 \pi] \times\{0\}$.

Pela continuidade uniforme, dado $0<\mathrm{G}<\mathrm{F}$, existe $v<v^{\prime}<\delta$ tal que

$$
\mathrm{t}+\mathrm{si} \in[0,2 \pi] \times[-v, v] \Longrightarrow\left|\operatorname{Re}\left(i \int_{0}^{t+i s} a(z) d z-i a_{0}(t+i s)\right)\right| \leq \mathrm{G}
$$

Se $v=$ Su, note que $e^{i n\left[\int_{0}^{t} a(r) d r-a_{0} t\right]} \in C^{\omega}(\mathbb{T})$, para todo $n \in \mathbb{Z}$ e com isso $v_{n} \in C^{\omega}(\mathbb{T})$, para todo $n \in \mathbb{Z}$, e além disto, para todo $t+s i \in[0,2 \pi] \times[-v, v], n \in \mathbb{Z}$, vale

$$
\begin{gathered}
\left|v_{n}(t+i s)\right|=\left|u_{n}(t+i s)\right|\left|e^{i n\left[\int_{0}^{t+i s} a(r) d r-a_{0}(t+i s)\right]}\right| \leq \\
\leq\left|u_{n}(t+i s)\right| e^{\left|\mathfrak{n} \| \operatorname{Re}\left(i\left[\int_{0}^{t+i s} a(r) d r-a_{0}(t+i s)\right]\right)\right|} \leq E e^{-|\mathfrak{n}| \mathrm{F}} e^{|\mathfrak{n}| G}=E e^{-|n|(F-G)},
\end{gathered}
$$

o que garante $v \in C^{\omega}\left(\mathbb{T}^{2}\right)$. Portanto, $S\left(C^{\omega}\left(\mathbb{T}^{2}\right)\right)=C^{\omega}\left(\mathbb{T}^{2}\right)$.

Proposição 6.16 Se $\mathrm{S}$, a e $\mathrm{a}_{0}$ são como na proposição anterior e $\mathrm{L}, \mathrm{M}: \mathcal{D}^{\prime}\left(\mathbb{T}^{2}\right) \rightarrow$ $\mathcal{D}^{\prime}\left(\mathbb{T}^{2}\right)$ são dados por $\mathrm{L}=\partial_{\mathrm{t}}+\mathrm{a}(\mathrm{t}) \partial_{x}$ e $\mathrm{M}=\partial_{\mathrm{t}}+\mathrm{a}_{0} \partial_{x}$, então $\mathrm{SL}=\mathrm{MS}$.

DemonstraÇÃo. Vamos calcular inicialmente MSu:

$$
\begin{gathered}
\partial_{t} S u=\sum_{n \in \mathbb{Z}} \partial_{t}\left(u_{n}(t) e^{i n\left[\int_{0}^{t} a(r) d r-a_{0} t\right]} e^{i n x}\right)= \\
=\sum_{n \in \mathbb{Z}}\left(\dot{u}_{n}(t)+n i\left(a(t)-a_{0}\right) u_{n}(t)\right) e^{i n\left[\int_{0}^{t} a(r) d r-a_{0} t\right]} e^{i n x} .
\end{gathered}
$$

Também,

$$
\begin{gathered}
a_{0} \partial_{x} S u=\sum_{n \in \mathbb{Z}} a_{0} \partial_{x}\left(u_{n}(t) e^{i n\left[\int_{0}^{t} a(r) d r-a_{0} t\right]} e^{i n x}\right)= \\
=\sum_{n \in \mathbb{Z}} i n a_{0}\left(u_{n}(t) e^{i n\left[\int_{0}^{t} a(r) d r-a_{0} t\right]} e^{i n x}\right) .
\end{gathered}
$$

Logo,

$$
M S u=\sum_{n \in \mathbb{Z}}\left(\dot{u}_{n}(t)+i n a(t) u_{n}(t)\right) e^{i n\left[\int_{0}^{t} a(r) d r-a_{0} t\right]} e^{i n x} .
$$

Para SLu, temos

$$
\begin{aligned}
\partial_{t} u & =\sum_{n \in \mathbb{Z}} \dot{u}_{n}(t) e^{i n x} \\
a(t) \partial_{x} u & =\sum_{n \in \mathbb{Z}} a(t) i n u_{n}(t) e^{i n x}
\end{aligned}
$$

e portanto,

$$
\mathrm{Lu}=\sum_{n \in \mathbb{Z}}\left(\dot{u}_{n}(t)+i n a(t) u_{n}(t)\right) e^{i n x} .
$$

Com isso concluímos $\mathrm{SL}=\mathrm{MS}$. 
Proposição 6.17 Sejam L e $M: \mathcal{D}^{\prime}\left(\mathbb{T}^{2}\right) \longrightarrow \mathcal{D}^{\prime}\left(\mathbb{T}^{2}\right)$ operadores lineares e $\mathrm{S}$ : $\mathcal{D}^{\prime}\left(\mathbb{T}^{2}\right) \longrightarrow \mathcal{D}^{\prime}\left(\mathbb{T}^{2}\right)$ uma bijeção que preserva $\mathrm{C}^{\omega}\left(\mathbb{T}^{2}\right)$. Suponha que MS $=\mathrm{SL}$. Então L é GAH se e somente se M é GAH.

DemonstraçÃo. Suponha que L seja GAH e que $M u=f, f \in C^{\omega}\left(\mathbb{T}^{2}\right)$. Então $M u=S L S^{-1} u=f$ implica que $L\left(S^{-1} u\right)=S^{-1} f$. Como $S^{-1} f \in C^{\omega}\left(\mathbb{T}^{2}\right)$ e L é $G A H$, obtemos $S^{-1} u \in C^{\omega}\left(\mathbb{T}^{2}\right)$ e com isso $S S^{-1} u=u \in C^{\omega}\left(\mathbb{T}^{2}\right)$, o que nos dá que $M$ é $G A H$.

Suponha agora que $M$ seja $G A H$ e que $L u=f, f \in C^{\omega}\left(\mathbb{T}^{2}\right)$. Então $L u=$ $S^{-1} M S u=f$ implica que $M(S u)=S f$. Como $S f \in C^{\omega}\left(\mathbb{T}^{2}\right)$ e $M$ é $G A H$, obtemos $\mathrm{Su} \in \mathrm{C}^{\omega}\left(\mathbb{T}^{2}\right)$ e com isso $\mathrm{S}^{-1} \mathrm{Su}=u \in \mathrm{C}^{\omega}\left(\mathbb{T}^{2}\right)$, o que nos dá que L é GAH.

Proposição 6.18 Seja $\Psi: \mathbb{R}^{2} \longrightarrow \mathbb{R}^{2}, \Psi(s, y)=(t, x)$, um difeomorfismo $C^{\infty}$ tal que

$\operatorname{Dado}(m, n) \in \mathbb{Z}^{2}$, existe $\alpha \in \mathbb{Z}^{2}$ tal que

$$
\Psi^{-1}((t, x)+2 \pi(m, n))=\Psi^{-1}(t, x)+2 \pi \alpha .
$$

Para toda distribuição $u \in \mathcal{D}^{\prime}\left(\mathbb{T}^{2}\right)$, defina $u \circ \Psi: \mathbb{C}^{\infty}\left(\mathbb{T}^{2}\right) \longrightarrow \mathbb{C}$ por

$$
(u \circ \Psi)(\phi)=\left(u,\left|J_{\Psi^{-1}}\right| \phi \circ \Psi^{-1}\right) .
$$

Então u० $\Psi=v \in \mathcal{D}^{\prime}\left(\mathbb{T}^{2}\right)$ e, além disto,

$$
\begin{aligned}
& \partial_{s} v=\partial_{s} t \partial_{t} v+\partial_{s} x \partial_{x} v \\
& \partial_{y} v=\partial_{y} t \partial_{t} v+\partial_{y} x \partial_{x} v .
\end{aligned}
$$

Demonstração. Devemos verificar primeiro que $\left|J_{\Psi^{-1}}\right| \phi \circ \Psi^{-1}$ é de fato um elemento de $C^{\infty}\left(\mathbb{T}^{2}\right)$. Note que $\mathrm{J}_{\Psi^{-1}} \neq 0$.

Temos que $\left|J_{\Psi^{-1}}\right| \phi \circ \Psi^{-1} \in \mathrm{C}^{\infty}\left(\mathbb{R}^{2}\right)$, pois $\Psi$ é um difeomorfismo $\mathrm{C}^{\infty}$.

Também, $\left|J_{\Psi^{-1}}\right| \phi \circ \Psi^{-1} \in C^{\infty}\left(\mathbb{R}^{2}\right)$ é $2 \pi$-periódica. De fato, verifiquemos primeiro que $J_{\Psi^{-1}}((t, x)+2 \pi(m, n))=J_{\Psi^{-1}}(t, x)$, para todo $(m, n) \in \mathbb{Z}^{2}$ e para todo $(t, x) \in$ $\mathbb{R}^{2}$. Seja $\Psi^{-1}(t, x)=\left(\Psi_{1}^{-1}(t, x), \Psi_{2}^{-1}(t, x)\right)$. De 6.19, seque que $\partial_{t} \Psi_{j}^{-1}, \partial_{x} \Psi_{j}^{-1}$ são $2 \pi$-periódicas, $j=1,2$, e com isso, $\left|J_{\Psi^{-1}}\right|$ é $2 \pi$-periódica.

A linearidade de $v$ é direta. A continuidade segue do fato de que, se $\phi$ tende a zero em $C^{\infty}\left(\mathbb{T}^{2}\right)$, então $\phi \circ \Psi^{-1}$ também o faz.

Por fim, de acordo com a definição de $v$, temos

$$
\left(\partial_{s} v, \phi\right)=\left(\nu, \partial_{s} \phi\right)=\left(u, \partial_{s} \phi \circ \Psi^{-1}\left|J_{\Psi^{-1}}\right|\right)=
$$




$$
\begin{gathered}
\left(u,\left(\partial_{t} \phi \circ \Psi^{-1} \partial_{s} t(s, y)+\partial_{x} \phi \circ \Psi^{-1} \partial_{s} x(s, y)\right)\left|J_{\Psi^{-1}}\right|\right)= \\
=\partial_{s} t \partial_{t} u+\partial_{s} x \partial_{x} u .
\end{gathered}
$$

De maneira análoga, concluímos que $\partial_{y} v=\partial_{y} t \partial_{t} u+\partial_{y} x \partial_{x} u$.

Proposição 6.20 Considere a mudança de variáveis

$$
(t, x)=\Psi(s, y)=(t(s, y), x(s, y)),
$$

onde $\Psi: \mathbb{R}^{2} \longrightarrow \mathbb{R}^{2}$ satisfaz as condições da proposição 6.18 e, além disto, $\Psi$ é um difeomorfismo analitico real, ou seja, cada uma das funções $\mathrm{t}(\mathrm{s}, \mathrm{y}), \mathrm{x}(\mathrm{s}, \mathrm{y})$, $s(t, x), y(t, x)$ é uma função analítica real de duas variáveis, a valores reais. Sejam L, M: $\mathcal{D}^{\prime}\left(\mathbb{T}^{2}\right) \longrightarrow \mathcal{D}^{\prime}\left(\mathbb{T}^{2}\right)$ dados por

$$
\begin{gathered}
\mathrm{Lu}=\partial_{\mathrm{t}} u+c(t) \partial_{x} u \\
M v=\mu(s, y) \partial_{s} v+\kappa(s, y) \partial_{y} v
\end{gathered}
$$

onde $\mathrm{c} \in \mathrm{C}^{\omega}(\mathbb{T}) e$

$$
\begin{gathered}
\mu(s, y)=\partial_{y} x(s, y)-c(t(s, y)) \partial_{y} t(s, y), \\
\kappa(s, y)=-\partial_{s} x(s, y)+c(t(s, y)) \partial_{s} t(s, y) .
\end{gathered}
$$

Então L é GAH se e somente se $M$ é GAH.

Demonstração. Faça $v=u \circ \Psi$. Da proposição 6.18 acima, obtemos

$$
\partial_{s} v=\partial_{s} t \partial_{t} v+\partial_{s} x \partial_{x} v
$$

e

$$
\partial_{y} v=\partial_{y} t \partial_{t} v+\partial_{y} x \partial_{x} v
$$

Defina $\zeta(s, y)=\partial_{y} x(s, y) \partial_{s} t(s, y)-\partial_{y} t(s, y) \partial_{s} x(s, y)=\left|J_{\Psi}(s, y)\right|$. Como $\Psi$ é um difeomorfismo, temos que $\zeta(s, y) \neq 0,(s, y) \in \mathbb{R}^{2}$. Note que $1 / \zeta \in C^{\omega}\left(\mathbb{R}^{2}, \mathbb{R}\right)$. Resolvendo o sistema acima, obtemos

$$
\partial_{x}(u \circ \Psi)=\frac{\partial_{s} t \partial_{y} v-\partial_{s} v \partial_{y} t}{\zeta}
$$

e

$$
\partial_{t}(u \circ \Psi)=\frac{\partial_{s} v \partial_{y} x-\partial_{s} x \partial_{y} v}{\zeta}
$$

Desta forma, temos

$$
\partial_{t}(u \circ \Psi)+c(t(s, y)) \partial_{x}(u \circ \Psi)=\zeta^{-1}(s, y)\left(\mu(s, y) \partial_{s} v+\kappa(s, y) \partial_{y} v\right)
$$


que nos garante que $M$ é GAH se e somente se $\zeta M$ é GAH.

Note que, se $v=u \circ \Psi$, então $L u=f$ se e somente se $\zeta M v=g \doteq f \circ \Psi$.

Agora suponha que L é GAH, e que $M v=h, h \in C^{\omega}\left(\mathbb{R}^{2}\right)$. Então $\zeta M v=\zeta h=g$, $g \in C^{\omega}\left(\mathbb{R}^{2}\right)$. Isso implica que $L u=f$, com $u=v \circ \Psi^{-1}, f=g \circ \Psi^{-1}, f \in C^{\omega}\left(\mathbb{T}^{2}\right)$ e, portanto, como L é GAH, temos que $u \in C^{\omega}\left(\mathbb{T}^{2}\right)$, e com isso $v \in C^{\omega}\left(\mathbb{T}^{2}\right)$, o que resulta em $M$ ser GAH.

Por outro lado, suponha que $M$ é GAH. Então $\zeta M$ é GAH. Se $L u=f$, com $f \in C^{\omega}\left(\mathbb{T}^{2}\right)$, então $\zeta M v=g$, com $g \in C^{\omega}\left(\mathbb{T}^{2}\right)$ e, portanto, $v \in C^{\omega}\left(\mathbb{T}^{2}\right)$, o que implica $u \in C^{\omega}\left(\mathbb{T}^{2}\right)$, ou seja, L é GAH.

Proposição 6.21 Se b $\in \mathrm{C}^{\omega}(\mathbb{T} ; \mathbb{R})$ muda de sinal então existe uma translação tal que $\mathrm{b}(\mathrm{t})=\mathrm{b}\left(\mathrm{t}+\mathrm{t}_{1}^{\prime}\right)$ satisfaz $\mathrm{b}(0)=0$ e $\max _{\mathrm{t} \in[0,2 \pi]}\left\{\int_{0}^{\mathrm{t}} \mathrm{b}(\mathrm{r}) \mathrm{dr}\right\}$ é atingido no interior de $[0,2 \pi]$.

Demonstração. Comece notando que se $b$ muda de sinal em $t_{0}$, então $t_{0}$ é um extremo local de $\mathrm{B}: \mathbb{R} \longrightarrow \mathbb{R}$, tal que $\mathrm{B}^{\prime}=\mathrm{b}$ pois neste caso encontramos $\delta>0$ tal que $B$ é crescente (ou decrescente) em $\left[t_{0}-\delta, t_{0}\right]$ e decrescente (ou crescente) em $\left[t_{0}, t_{0}+\delta\right]$.

Também vale que B possui pelo menos um mínimo em $[0,2 \pi]$. De fato, se $t_{0}$ é um mínimo, não há nada a fazer. Se $t_{0}$ é um máximo então $t_{0}+2 \pi$ também é. Como a derivada é contínua, entre dois máximos existe um mínimo, pois $\mathrm{b}$ possui sinais opostos à direita de $t_{0}$ e à esquerda de $t_{0}+2 \pi$, garantindo que existe $t_{1} \in\left(t_{0}, t_{0}+2 \pi\right)$ onde $b$ muda de sinal de um jeito contrário àquele em $t_{0}$. Se $t_{1} \in\left(2 \pi, 2 \pi+t_{0}\right)$, então pela proposição $t_{1}^{\prime}=t_{1}-2 \pi$ é o mínimo procurado; se $t_{1} \in\left(t_{0}, 2 \pi\right]$, faça $t_{1}^{\prime}=t_{1}$.

Para ver que $b(t)=b\left(t+t_{1}^{\prime}\right)$ satisfaz as condições desejadas, note que nem 0 nem $2 \pi$ são pontos de máximo de $B(t)=B\left(t+t_{1}^{\prime}\right)-B\left(t_{1}^{\prime}\right)$, pois são pontos de mínimo.

\subsubsection{Majoração de Funções Analíticas Reais}

A proposição 6.23 garante que podemos comparar funções analíticas reais de uma variável de um jeito parecido com o qual comparamos polinômios.

Proposição 6.22 Seja $f:(-a, a) \longrightarrow \mathbb{R}, \operatorname{com} f(x)=\sum_{j=n}^{\infty} a_{j} x^{j}, a_{n} \neq 0, n \geq 1$. Então

1. $f(x)$ muda de sinal de - para $+e m x=0 \Longleftrightarrow n$ é impar e $a_{n}>0$,

2. $\mathrm{f}(\mathrm{x})$ muda de sinal de + para $-e m \mathrm{x}=0 \Longleftrightarrow \mathrm{n}$ é impar e $\mathrm{a}_{\mathrm{n}}<0$,

3. $x=0$ é um máximo $\Longleftrightarrow n$ é par e $a_{n}<0$, 
4. $x=0$ é um minimo $\Longleftrightarrow n$ é par e $\mathrm{a}_{\mathrm{n}}>0$.

DEMONSTRAÇÃo. Escreva

$$
f(x)=a_{n} x^{n}(1+\alpha(x)), \text { onde } \alpha(x)=a_{n}^{-1} \sum_{j=n+1}^{\infty} a_{j} x^{j-n} .
$$

Como $\lim _{x \rightarrow 0} \alpha(x)=0$, existe $\delta>0$ tal que

$$
|x|<\delta \Longrightarrow 1+\alpha(x)>0
$$

Portanto, $f(x)$ tem o mesmo sinal que $a_{n} x^{n}$ em $(-\delta, \delta)$. Em seguida, note que $x=0$ é um ponto de máximo se e somente se $f(x) \leq 0$ numa vizinhança do zero, e que $x=0$ é um ponto de mínimo, se somente se $f(x) \geq 0$ numa vizinhança do zero.

Proposição 6.23 Sejam $\mathrm{f}, \mathrm{g}:(-\mathrm{a}, \mathrm{a}) \rightarrow \mathbb{R} \mathrm{com}$

$$
f(x)=\sum_{j=n}^{\infty} a_{j} x^{j}, \quad g(x)=\sum_{j=m}^{\infty} b_{j} x^{j},
$$

$\mathrm{n}, \mathrm{m} \in \mathbb{N}, \mathrm{a}_{\mathrm{n}} \neq 0, \mathrm{~b}_{\mathrm{m}} \neq 0, \mathrm{~m} \geq 1$ e $\mathrm{n} \geq \mathrm{m}$. Então existem $\mathrm{K}, \delta>0$ tais que $|\mathrm{f}(\mathrm{x})| \leq \mathrm{K}|\mathrm{g}(\mathrm{x})|, \forall x \in[-\delta, \delta]$.

Demonstração. Defina $\tilde{f}(x)=\sum_{j=n}^{\infty} a_{j} x^{j-n}, \tilde{g}(x)=\sum_{j=m}^{\infty} b_{j} x^{j-m}$. Desta forma, temos $\tilde{f}(0) \neq 0, \tilde{g}(0) \neq 0$ e, se $1>\delta>0$ é tal que $\tilde{g}$ não se anula em $[-\delta, \delta]$, então $\min _{x \in[-\delta, \delta]}\{|\tilde{g}(x)|\} \neq 0$. Assim existe $K>0$ satisfazendo

$$
\begin{gathered}
|\boldsymbol{f}(x)|=\left|x^{\mathfrak{n}}\right||\tilde{\mathbf{f}}(x)| \leq\left|\chi^{\mathfrak{n}}\right| \max _{x \in[-\delta, \delta]}\{|\tilde{f}(x)|\} \leq \\
\leq \mathrm{K}\left|\chi^{\mathfrak{n}}\right| \min _{x \in[-\delta, \delta]}\{|\tilde{\mathfrak{g}}(x)|\} \leq \mathrm{K}\left|\chi^{\mathfrak{m}}\right||\tilde{\boldsymbol{g}}(x)|=\mathrm{K}|\mathrm{g}(x)|
\end{gathered}
$$

para todo $x \in[-\delta, \delta]$.

\subsubsection{Caso de Coeficientes Constantes}

Considere um operador diferencial parcial, com coeficientes constantes, em $\mathcal{D}^{\prime}\left(\mathbb{T}^{2}\right)$,

$$
P=\sum_{|\alpha| \leq M} a_{\alpha} D^{\alpha}
$$

onde $\alpha=\left(\alpha_{1}, \alpha_{2}\right) \in \mathbb{N}^{2}, a_{\alpha} \in \mathbb{C}$.

Se $u, f \in \mathcal{D}^{\prime}\left(\mathbb{T}^{2}\right)$ são dadas por suas séries de Fourier 


$$
\begin{aligned}
& u=\sum_{(m, n) \in \mathbb{Z}^{2}} c_{(m, n)}(u) e^{i(n t+m x)} \\
& f=\sum_{(m, n) \in \mathbb{Z}^{2}} c_{(m, n)}(f) e^{i(n t+m x)},
\end{aligned}
$$

então, como a derivação parcial comuta com a série de Fourier, vale

$$
\begin{gathered}
\mathrm{Lu}=\mathrm{f} \Longleftrightarrow \sum_{(\mathrm{m}, \mathfrak{n}) \in \mathbb{Z}^{2}} \sum_{|\alpha| \leq M} \mathrm{a}_{\alpha} \mathrm{n}^{\alpha_{1}} \mathrm{~m}^{\alpha_{2}} \mathrm{c}_{(\mathrm{m}, \mathfrak{n})}(\mathrm{u}) \mathrm{e}^{\mathrm{i}(\mathrm{nt}+\mathrm{m} x)}= \\
=\sum_{(\mathfrak{m}, \mathfrak{n}) \in \mathbb{Z}^{2}} \mathrm{c}_{(\mathrm{m}, \mathfrak{n})}(\mathrm{f}) \mathrm{e}^{\mathrm{i}(\mathrm{nt}+\mathrm{m} x)} \Longleftrightarrow \\
\Longleftrightarrow \sum_{|\alpha| \leq M} \mathrm{a}_{\alpha} \mathrm{n}^{\alpha_{1}} \mathrm{~m}^{\alpha_{2}} \mathrm{c}_{(\mathfrak{m}, \mathfrak{n})}(\mathrm{u})=\mathrm{c}_{(\mathrm{m}, \mathfrak{n})}(\mathrm{f}), \quad \forall(\mathrm{m}, \mathrm{n}) \in \mathbb{Z}^{2} .
\end{gathered}
$$

Defina $P(m, n)=\sum_{|\alpha| \leq M} a_{\alpha} n^{\alpha_{1}} m^{\alpha_{2}}$ (o símbolo do operador $P$ ). Então vale

$$
P(m, n) c_{(m, n)}(u)=c_{(m, n)}(f) .
$$

O próximo teorema devido a Greenfield [G] traduz a hipoeliticidade analítica global, numa condição de crescimento no símbolo do operador.

Teorema 6.24 Considere $\mathrm{P}$ como acima. $\mathrm{P}$ é $G A H$ se e somente, dado $\mathrm{K}>0$ existe $\mathrm{N}_{\mathrm{K}}>0$ tal que

$$
|P(m, n)| \geq e^{-K\left(m^{2}+n^{2}\right)^{\frac{1}{2}}}, \quad|n|,|m| \geq N_{K}
$$

Demonstração. Suponha que vale a desigualdade 6.25. Note que, se $g \in \mathcal{D}^{\prime}\left(\mathbb{T}^{2}\right)$ então $g \in C^{\omega}\left(\mathbb{T}^{2}\right)$ se e somente se existem constantes $\beta>0, \gamma>0$, tais que $\left|\mathfrak{c}_{(\mathfrak{m}, \mathfrak{n})}(\mathrm{g})\right| \leq \gamma e^{-\beta|(\mathfrak{m}, \mathfrak{n})|}$. Seja então $u \in \mathcal{D}^{\prime}\left(\mathbb{T}^{2}\right)$ e $f \in \mathrm{C}^{\omega}\left(\mathbb{T}^{2}\right)$ tais que $\mathrm{Pu}=\mathrm{f}$. Então existem constantes $\beta>0, \gamma>0$ tais que

$$
\left|\mathfrak{c}_{(m, n)}(f)\right| \leq \gamma e^{-\beta\left(m^{2}+n^{2}\right)^{\frac{1}{2}}} .
$$

Tome $0<K<\beta$. Então, se $|n|,|m| \geq N_{K}$, temos

$$
\left|c_{(m, n)}(u)\right|=\frac{\left|c_{(m, n)}(f)\right|}{|P(m, n)|} \leq \frac{\gamma e^{-\beta\left(m^{2}+n^{2}\right)^{\frac{1}{2}}}}{e^{-K\left(m^{2}+n^{2}\right)^{\frac{1}{2}}}} \leq \gamma e^{(-\beta+K)\left(m^{2}+n^{2}\right)^{\frac{1}{2}}} .
$$

Seja $\gamma^{\prime}>0$ tal que $\left|\mathfrak{c}_{(m, n)}(u)\right| \leq \gamma^{\prime} e^{(-\beta+K)\left(m^{2}+n^{2}\right)^{\frac{1}{2}}}$, para todo $(m, n) \in \mathbb{Z}^{2}$ tal que $|\mathrm{n}|,|\mathrm{m}|<\mathrm{N}_{\mathrm{K}}$. Tome $\mathrm{C}=\max \left\{\gamma, \gamma^{\prime}\right\}$. Então

$$
\left|\mathfrak{c}_{(\mathrm{m}, \mathfrak{n})}(\mathrm{u})\right| \leq \mathrm{Ce}^{(-\beta+K)\left(\mathrm{m}^{2}+\mathrm{n}^{2}\right)^{\frac{1}{2}}},
$$


para todo $(m, n) \in \mathbb{Z}^{2}$, o que significa que $u \in C^{\omega}\left(\mathbb{T}^{2}\right)$ e L é GAH.

Reciprocamente, se a desigualdade 6.25 não vale, então existem $K_{0}>0$ e uma sequência $\left(m_{j}, n_{j}\right) \in \mathbb{Z}^{2}, j \in \mathbb{N}$, onde $\left|n_{j}\right| \longrightarrow \infty$, tais que

$$
\left|P\left(m_{j}, n_{j}\right)\right| \leq e^{-K_{0}\left(m_{j}^{2}+n_{j}^{2}\right)^{\frac{1}{2}}} \text {. }
$$

Considere $u=\sum_{j \in \mathbb{N}} e^{i\left(n_{j} t+m_{j} x\right)}$, ou seja, $c_{(m, n)}(u)=1$, se existe $j_{0} \in \mathbb{N}$, tal que $(m, n)=\left(n_{j_{0}}, m_{j_{0}}\right)$ e $c_{(m, n)}(u)=0$, caso contrário. Seja $f=$ Lu. Desta forma, $\mathfrak{c}_{(\mathfrak{m}, n)}(u)$ forma uma sequência de crescimento lento, mas não decai exponencialmente (não converge pra zero), ou seja, $u \in \mathcal{D}^{\prime}\left(\mathbb{T}^{2}\right) \backslash C^{\omega}\left(\mathbb{T}^{2}\right)$. Mas note que $c_{(m, n)}(f)=$ $P(m, n)$, se existe $j_{0} \in \mathbb{N}$, tal que $(m, n)=\left(n_{j_{0}}, m_{j_{0}}\right)$ e $c_{(m, n)}(f)=0$, caso contrário. Portanto,

$$
\left|\mathfrak{c}_{(m, n)}(f)\right| \leq e^{-K_{0}\left(m^{2}+n^{2}\right)^{\frac{1}{2}}}
$$

para todo $(m, n) \in \mathbb{Z}^{2}$, o que implica em $f \in C^{\omega}\left(\mathbb{T}^{2}\right)$ e, portanto, P não é GAH.

O teorema principal da dissertação (6.3), quando considerado apenas o caso de o operador ter coeficientes constantes, adquire o seguinte enunciado.

Teorema 6.26 Considere o operador em $\mathcal{D}^{\prime}\left(\mathbb{T}^{2}\right), \quad L=\partial_{t}+(a+i b) \partial_{x}$, $\mathrm{a}, \mathrm{b} \in \mathbb{R}$. Então L é globalmente analítico hipoelítico se e somente se vale uma das afirmações

1. $b \neq 0$;

2. $\mathrm{b}=0$ e a é um número irracional não exponencial Liouville.

Demonstração. Mostremos que se $b \neq 0$ então L é GAH.

Colocando o operador em questão na notação utilizada no teorema anterior, temos que $L=i D^{(1,0)}+i(a+b i) D^{(0,1)}$, e com isso,

$$
|\mathrm{L}(\mathrm{m}, \mathrm{n})|=|\mathfrak{i m}+\mathfrak{i}(\mathrm{a}+\mathrm{ib}) \mathrm{n}|=|\mathrm{m}+\mathrm{an}+\mathrm{ibn}| .
$$

Se $(m, n) \in \mathbb{Z}^{2}$ é tal que $|n b| \geq 1$ então

$$
|\mathrm{L}(\mathrm{m}, \mathrm{n})|^{2}=(\mathrm{m}+\mathrm{an})^{2}+(\mathrm{bn})^{2} \geq(\mathrm{bn})^{2} \geq 1 \geq \mathrm{e}^{-\left(\mathrm{m}^{2}+\mathrm{n}^{2}\right)^{\frac{1}{2}}},
$$

e o teorema anterior garante a afirmação.

Mostremos agora que se $b=0$ então L é GAH se e somente se a é irracional e não é exponencial Liouville. Equivalentemente, L não é GAH se e somente se a é racional ou a é um número exponencial Liouville (irracional)

Suponha agora que $b=0$. Mostremos que se a é racional ou a é um número exponencial Liouville (irracional) então L não é GAH. 
Suponha que $a=p / q p, q \in \mathbb{Z}, q \geq 1$. Então, se $r \in \mathbb{Z}$, temos

$$
\mathrm{L}(\mathrm{rp},-\mathrm{rq})=\mathrm{i}\left(\mathrm{rp}-\frac{\mathrm{p}}{\mathrm{q}} \mathrm{rq}\right)=0,
$$

ou seja, $\mathrm{L}(\mathrm{m}, \mathrm{n})$ tem uma infinidade de zeros, e com isso não vale a desigualdade 6.25 do teorema anterior, ou seja, L não é GAH.

Suponha agora que a é exponencial Liouville. Então existe $\varepsilon>0$ e uma sequência $\left(m_{j}, n_{j}\right) \in \mathbb{Z}^{2}$, com $n_{j}>0$, para todo $j \in \mathbb{N}, m_{j}$ e $n_{j}$ primos entre si e $n_{j} \longrightarrow \infty$, onde vale

$$
\left|a+\frac{m_{j}}{n_{j}}\right| \leq e^{-\varepsilon n_{j}}
$$

Portanto, $\left|m_{j}+n_{j} a\right| \leq\left|n_{j}\right| e^{-\varepsilon n_{j}}$. Note também que como $n_{j} e^{-\varepsilon n_{j} / 2} \rightarrow 0$, existe $C_{1}>0$ tal que

$$
\left|\mathrm{L}\left(\mathrm{m}_{\mathrm{j}}, \mathrm{n}_{\mathrm{j}}\right)\right| \leq \mathrm{n}_{\mathrm{j}} \mathrm{e}^{-\varepsilon n_{\mathrm{j}}} \leq \mathrm{C}_{1} \mathrm{e}^{-\varepsilon n_{\mathrm{j}} / 2} .
$$

Para ver que isso implica que L não é GAH, tome $u \in \mathcal{D}^{\prime}\left(\mathbb{T}^{2}\right) \backslash C^{\omega}\left(\mathbb{T}^{2}\right)$, com

$$
c_{(m, n)}(u)=\left\{\begin{array}{ll}
1, & \text { se }(m, n)=\left(m_{j}, n_{j}\right) \\
0, & \text { caso contrário }
\end{array} .\right.
$$

Note que realmente $u \in \mathcal{D}^{\prime}\left(\mathbb{T}^{2}\right) \backslash C^{\omega}\left(\mathbb{T}^{2}\right)$, pois $c_{(m, n)}(u)$ forma uma sequência de crescimento lento, mas não decai exponencialmente. Desta forma, se $f=$ Lu então

$$
c_{(m, n)}(f)=\left\{\begin{array}{l}
L\left(m_{j}, n_{j}\right), \quad \text { se }(m, n)=\left(m_{j}, n_{j}\right) \\
0, \quad \text { caso contrário }
\end{array} .\right.
$$

Note que como $n_{j} \rightarrow+\infty$, podemos supor que $C_{1} e^{-\varepsilon n_{j} / 2}<1$. Deste modo,

$$
\left|m_{j}\right|-|a| n_{j} \leq\left|a n_{j}+m_{j}\right|=\left|L\left(m_{j}, n_{j}\right)\right| \leq C_{1} e^{-\varepsilon n_{j} / 2} \leq 1 \leq n_{j} .
$$

Logo, $\left|m_{j}\right| \leq(1+|a|) n_{j}$ e daí,

$$
\left|\left(m_{j}, n_{j}\right)\right|=\left(m_{j}^{2}+n_{j}^{2}\right)^{\frac{1}{2}} \leq\left((1+|a|)^{2} n_{j}^{2}+n_{j}^{2}\right)^{\frac{1}{2}}=\left((1+|a|)^{2}+1\right)^{\frac{1}{2}} n_{j} .
$$

Colocando

$$
\varepsilon^{\prime}=\frac{\varepsilon}{2\left((1+|a|)^{2}+1\right)^{\frac{1}{2}}},
$$

obtemos

$$
\varepsilon^{\prime}\left|\left(m_{j}, n_{j}\right)\right| \leq \frac{\varepsilon}{2} n_{j}
$$

Assim,

$$
c_{\left(m_{j}, n_{j}\right)}(f)=\left|L\left(m_{j}, n_{j}\right)\right| \leq C_{1} e^{-\frac{\varepsilon}{2} n_{j}} \leq C_{1} e^{-\varepsilon^{\prime}\left|\left(m_{j}, n_{j}\right)\right|} .
$$

Como $c_{(m, n)}(f)=0$ se $(m, n) \neq\left(m_{j}, n_{j}\right)$ para todo $j$ vemos que

$$
\left|\mathfrak{c}_{(m, n)}(f)\right| \leq C_{1} e^{-\varepsilon^{\prime}|(m, n)|} .
$$


Reciprocamente, suponha que L não é GAH. Resta mostrar que se $b=0$ e a é irracional então a é exponencial Liouville.

Como não vale 6.25 , existem $K_{0}>0$ e uma sequência $\left(m_{j}, n_{j}\right) \in \mathbb{Z}^{2}, j \in \mathbb{N}$, onde $\left|n_{j}\right| \longrightarrow \infty,\left|m_{j}\right| \longrightarrow \infty, j \longrightarrow \infty$, e vale

$$
\begin{gathered}
\left|m_{j}+a n_{j}\right|=\left|L\left(m_{j}, n_{j}\right)\right| \leq e^{-K_{o}\left(m_{j}^{2}+n_{j}^{2}\right)^{\frac{1}{2}}} \leq e^{-K_{o}\left|n_{j}\right|} \Longrightarrow \\
\Longrightarrow\left|a+\frac{m_{j}}{n_{j}}\right| \leq\left|n_{j}\right|^{-1} e^{-K_{0}\left|n_{j}\right|} \leq e^{-K_{o}\left|n_{j}\right|} .
\end{gathered}
$$

Colocando $p_{j}=-\sin a l\left(n_{j}\right) m_{j}$ e $q_{j}=\left|n_{j}\right|$ obtemos,

$$
\left|a-\frac{p_{j}}{q_{j}}\right| \leq e^{-K_{0} q_{j}}
$$

ou seja, a é exponencial Liouville.

\subsection{Demonstração do Teorema Principal}

Para uma melhor compreensão da demonstração, vamos analisar as implicações envolvidas no enunciado do teorema 6.3.

Como no teorema, sejam $L: \mathcal{D}^{\prime}\left(\mathbb{T}^{2}\right) \longrightarrow \mathcal{D}^{\prime}\left(\mathbb{T}^{2}\right), L=\partial_{t}+c(t) \partial_{x}$, onde $c \in C^{\omega}(\mathbb{T})$, $c(t)=a(t)+i b(t), e a, b \in C^{\omega}(\mathbb{T} ; \mathbb{R}), a_{0}=(2 \pi)^{-1} \int_{0}^{2 \pi} a(t) d t, b_{0}=(2 \pi)^{-1} \int_{0}^{2 \pi} b(t) d t$. Defina

$\mathrm{P}_{1}=\mathrm{L}$ é $\mathrm{GAH}$.

$P_{2}=b$ é a função nula.

$\mathrm{P}_{3}=\mathrm{b}$ muda de sinal.

$P_{4}=a_{0} \in \mathbb{Q}$

$P_{5}=a_{0}$ é exponencial Liouville.

Assim, o teorema 6.3 tem a forma

$$
\mathrm{P}_{1} \Longleftrightarrow\left(\neg \mathrm{P}_{2} \wedge \neg \mathrm{P}_{3}\right) \vee\left(\mathrm{P}_{2} \wedge\left(\neg \mathrm{P}_{4} \wedge \neg \mathrm{P}_{5}\right)\right)
$$

que é equivalente a

$$
\left(\neg \mathrm{P}_{2} \Rightarrow\left(\mathrm{P}_{1} \Leftrightarrow \neg \mathrm{P}_{3}\right)\right) \wedge\left(\mathrm{P}_{2} \Rightarrow\left(\mathrm{P}_{1} \Leftrightarrow \neg \mathrm{P}_{4} \wedge \neg \mathrm{P}_{5}\right)\right)
$$

Para demonstrar o teorema, vamos verificar que vale a fórmula (6.28), através das implicações

$$
\mathrm{P}_{2} \Rightarrow\left(\mathrm{P}_{1} \Leftrightarrow \neg \mathrm{P}_{4} \wedge \neg \mathrm{P}_{5}\right)
$$




$$
\begin{aligned}
& \neg \mathrm{P}_{2} \Rightarrow\left(\neg \mathrm{P}_{3} \Rightarrow \mathrm{P}_{1}\right), \\
& \neg \mathrm{P}_{2} \Rightarrow\left(\mathrm{P}_{3} \Rightarrow \neg \mathrm{P}_{1}\right) .
\end{aligned}
$$

Em Português,

(6.29) Se b é a função nula então $L$ é $G A H$ se e somente se $a_{0} \in \mathbb{R} \backslash \mathbb{Q}$ e $a_{0}$ não é exponencial Liouville.

(6.30) Se b não é a função nula e b não muda de sinal então L é GAH.

(6.31) Se b muda de sinal então L não é GAH.

No caso de (6.31), apresentamos duas demonstrações diferentes, uma usando o método da fase estacionária e outra usando o método da maior descida.

A implicação mais simples é a primeira, (6.29).

Se b é a função nula então $\mathrm{L}$ é $G A H$ se e somente se $\mathrm{a}_{0} \in \mathbb{R} \backslash \mathbb{Q}$ e $\mathrm{a}_{0}$ não é exponencial Liouville.

Se $b$ é a função nula então $L=\partial_{t}+a(t) \partial_{x}$. Usando as proposições $6.16,6.17$ e o teorema 6.26, segue que L é GAH se e somente se $M=\partial_{t}+a_{0} \partial_{x}$ é GAH. Mas $M$ é GAH se e somente se $a_{0} \in \mathbb{R} \backslash \mathbb{Q}$ e $a_{0}$ não é exponencial Liouville.

Vejamos agora que vale (6.30).

Se b não é a função nula e b não muda de sinal então L é GAH.

Suponha $u \in \mathcal{D}^{\prime}\left(\mathbb{T}^{2}\right)$ e $L u=f \in C^{\omega}\left(\mathbb{T}^{2}\right)$. Se $f_{n} \in C^{\omega}(\mathbb{T}), n \in \mathbb{Z}$, é o $n-$ ésimo coeficiente parcial de Fourier de $f$, existem constantes $E, F, \delta_{1}>0$ tais que $\left|f_{n}(t+i w)\right| \leq E e^{-F|n|}, n \in \mathbb{Z}, t+i w \in[0,2 \pi] \times\left(-\delta_{1}, \delta_{1}\right)$.

Caso 1: b é não negativa.

Como $b$ é não negativa, não nula, e $b \in \mathbb{C}^{\omega}(\mathbb{T} ; \mathbb{R})$, temos $b_{0}>0$.

Se $n>0$, note que

$$
\left|e^{i 2 \pi n c_{0}}-1\right| \geq 1-\left|e^{i 2 \pi n c_{0}}\right|=1-e^{-2 \pi b_{0} n} \geq 1-e^{-2 \pi b_{0}}>0,
$$

portanto,

$$
\left|\left(e^{i 2 \pi n c_{0}}-1\right)^{-1}\right| \leq\left(1-e^{-2 \pi b_{0}}\right)^{-1} .
$$

Seja 


$$
\psi(t, s)=i \int_{t}^{t+s} c(r) d r=-\int_{t}^{t+s} b(r) d r+i \int_{t}^{t+s} a(r) d r .
$$

Como $b$ é não negativa, $B(t)=\int_{0}^{t} b(r) d r$ é crescente e, portanto,

$$
\operatorname{Re} \psi(t, s) \leq 0, \quad(t, s) \in[0,2 \pi] \times[0,2 \pi] .
$$

Note agora que $\psi$ é uma função analítica real de duas variáveis e, além disto, Re $\psi$ é uniformemente contínua em qualquer subconjunto compacto. Portanto, existe $\delta_{2}>0$ tal que $\psi(t+i w, s)$ está definida para todo $(t+i w, s)$ tal que $|w| \leq \delta_{2}$, $\mathrm{t} \in[0,2 \pi], \mathrm{s} \in[0,2 \pi]$ e, além disto, dado $0<\varepsilon<\mathrm{F}$, vale

$$
|\operatorname{Re} \psi(t+i w, s)-\operatorname{Re} \psi(t, s)|<\varepsilon
$$

e portanto

$$
\operatorname{Re} \psi(t+i w, s) \leq \operatorname{Re} \psi(t, s)+\varepsilon \leq \varepsilon .
$$

De acordo com o discutido acima, se $b$ é não negativa, $n>0$ e $\delta_{3}=\min \left\{\delta_{1}, \delta_{2}\right\}$, segue que, se $t+i \mathfrak{w} \in[0,2 \pi] \times\left[-\delta_{3}, \delta_{3}\right]$ então, pelo lema 6.8 ,

$$
\begin{gathered}
\left|u_{n}(t+i w)\right|=\left|\left(e^{i 2 \pi n c_{0}}-1\right)^{-1}\right|\left|\int_{0}^{2 \pi} e^{i n \int_{t+i w}^{t+i w+s} c(\sigma) d \sigma} f_{n}(t+i w+s) d s\right| \leq \\
\leq\left(1-e^{-2 \pi b_{0}}\right)^{-1} \int_{0}^{2 \pi}\left|f_{n}(t+i w+s) \| e^{i n \int_{t+i w}^{t+i w+s} c(\sigma) d \sigma}\right| d s= \\
=\left(1-e^{-2 \pi b_{0}}\right)^{-1} \int_{0}^{2 \pi}\left|f_{n}(t+i w+s)\right| e^{n \operatorname{Re} \psi(t+i w, s)} d s \leq \\
\leq\left(1-e^{-2 \pi b_{0}}\right)^{-1} E e^{-F n} 2 \pi e^{\varepsilon n} \leq 2 \pi\left(1-e^{-2 \pi b_{0}}\right)^{-1} E e^{-(F-\varepsilon) n} .
\end{gathered}
$$

Suponha agora $n<0$.

Então vale

$$
\left|1-e^{-i 2 \pi n c_{0}}\right| \geq 1-e^{2 \pi b_{0} n} \geq 1-e^{-2 \pi b_{0}}>0
$$

e, portanto,

$$
\left|\left(1-e^{-i 2 \pi n c_{0}}\right)^{-1}\right| \leq\left(1-e^{-2 \pi b_{0}}\right)^{-1} .
$$

Defina

$$
\xi(t, s)=i \int_{t-s}^{t} c(r) d r=-\int_{t-s}^{t} b(r) d r+i \int_{t-s}^{t} a(r) d r .
$$

Como $B(t)$ é crescente,

$$
\operatorname{Re} \xi(t, s) \leq 0, \quad(t, s) \in[0,2 \pi] \times[0,2 \pi]
$$

Assim como anteriormente, existe $\delta_{4}>0$ tal que $\xi(t+i w, s)$ está definida para todo $(t+i w, s)$ tal que $|w| \leq \delta_{4}, t \in[0,2 \pi], s \in[0,2 \pi]$, e vale 


$$
|\operatorname{Re} \xi(t+i w, s)-\operatorname{Re} \xi(t, s)|<\varepsilon
$$

que implica em

$$
\operatorname{Re} \xi(t+i w, s) \leq \operatorname{Re} \xi(t, s)+\varepsilon \leq \varepsilon .
$$

Podemos então concluir que, se $n<0, \delta_{5}=\min \left\{\delta_{1}, \delta_{4}\right\}$ e $t+i \mathfrak{i} \in[0,2 \pi] \times\left[-\delta_{5}, \delta_{5}\right]$ então

$$
\begin{gathered}
\left|u_{n}(t+i w)\right|=\left|\left(e^{i 2 \pi n c_{0}}-1\right)^{-1} \| \int_{0}^{2 \pi} e^{|\mathfrak{n}| \xi(t+i w)} f_{n}(t+i w+s) d s\right| \leq \\
\leq\left(1-e^{-2 \pi b_{0}}\right)^{-1}\left|f_{n}(t+i w+s)\right| \int_{0}^{2 \pi}\left|e^{|n| \xi(t+i w)}\right| d s= \\
=\left(1-e^{-2 \pi b_{0}}\right)^{-1}\left|f_{n}(t+i w+s)\right| \int_{0}^{2 \pi} e^{|\mathfrak{n}| \operatorname{Re} \psi(t+i w, s)} d s \leq \\
\leq\left(1-e^{-2 \pi b_{0}}\right)^{-1} E e^{-F|n|} 2 \pi e^{\varepsilon|n|} \leq 2 \pi\left(1-e^{-2 \pi b 0}\right)^{-1} E e^{-(F-\varepsilon)|n|} .
\end{gathered}
$$

Caso 2: b é não positiva.

Basta considerar o roteiro acima, mas agora utilizando as soluções $u_{n}(t)$ na forma 6.10 para o caso $n>0$, e as soluções na forma 6.11 para $n<0$.

A demonstração de (6.31) será feita a seguir.

\subsubsection{Soluções Singulares}

Chegamos aqui na parte mais lenta do teorema. Vamos mostrar que vale (6.31), ou seja, devemos mostrar que L não é GAH quando b muda de sinal, ou seja, que existe $f \in C^{\omega}\left(\mathbb{T}^{2}\right), u \in \mathcal{D}^{\prime}\left(\mathbb{T}^{2}\right) \backslash C^{\omega}\left(\mathbb{T}^{2}\right)$ tais que $L u=f$.

Como mencionado anteriormente, construiremos uma tal solução singular de dois modos.

Construção de uma Solução Singular pelo Método da Fase Estacionária Coloque

$$
A(t)=\int_{0}^{t} a(r) d r, \quad B(t)=\int_{0}^{t} b(r) d r \quad e \quad C(t)=\int_{0}^{t} c(r) d r .
$$

Vamos mostrar primeiro que no caso em que $b_{0} \neq 0$ é suficiente considerarmos o caso em que valem as seguintes condições:

1. Existe $t^{*} \in(0,2 \pi)$, com $B\left(t^{*}\right)=\max _{t \in[0,2 \pi]}\{B(t)\}>0$ e a origem é um mínimo local de $B$, (portanto, $b(0)=0)$.

2. $b_{0}<0$. 
Basta exibirmos uma mudança de variáveis analítica real de modo que o operador $M$ da proposição 6.20 tenha as propriedades exigidas acima.

Para obtermos 1, considere a proposição 6.21.

Podemos supor que $\mathrm{B}(0)=0$.

Se o dado $L$ não satisfaz 1 , considere a mudança de variáveis $\Psi_{1}: \mathbb{R}^{2} \longrightarrow \mathbb{R}^{2}$, $\Psi_{1}(s, y)=\left(s+t_{1}^{\prime}, y\right)=(t, x)$, onde $t_{1}^{\prime} \in[0,2 \pi)$ é um ponto de mínimo de $B$ (veja a demonstração da proposição 6.21). Assim, $M$ é tal que

$$
M=\partial_{s}+\tilde{c}(s) \partial_{y}
$$

onde $\tilde{\mathrm{c}}(\mathrm{s})=\mathrm{c}\left(\mathrm{s}+\mathrm{t}_{1}^{\prime}\right)$. Desta forma, $\tilde{\mathrm{B}}(\mathrm{s})=\int_{0}^{s} \tilde{\mathrm{b}}(\mathrm{r}) \mathrm{dr}$ satisfaz 1 para algum $\mathrm{t}^{*}$.

Assim, podemos assumir que 1 está satisfeita.

Agora, se o dado L não satisfaz 2, isto é, $b_{0}>0$, pois $b_{0} \neq 0$, considere a mudança de variáveis $\Psi_{2}: \mathbb{R}^{2} \longrightarrow \mathbb{R}^{2}, \Psi_{2}(s, y)=(-s, y)=(t, x)$. Neste caso,

$$
M=\partial_{s}-c(-s) \partial_{y}=\partial_{s}+\tilde{c}(s) \partial_{y}
$$

onde $\tilde{c}(s)=-c(-s)$. Note que

$$
\begin{gathered}
2 \pi \tilde{b}_{0}=\int_{0}^{2 \pi} \tilde{b}(r) d r=-\int_{0}^{2 \pi} b(-r) d r= \\
=\int_{0}^{-2 \pi} b(s) d s=-\int_{-2 \pi}^{0} b(s) d s=-2 \pi b_{0}<0 .
\end{gathered}
$$

Note que

$$
\tilde{\mathrm{B}}(\mathrm{s})=\int_{0}^{\mathrm{s}} \tilde{\mathrm{b}}(\mathrm{r}) \mathrm{dr}=\int_{0}^{-\mathrm{s}} \mathrm{b}(\mathrm{r}) \mathrm{dr}=\mathrm{B}(-\mathrm{s}) .
$$

Assim, a origem é um mínimo de $\tilde{\mathrm{B}}, \tilde{\mathrm{B}}(0)=0$ e, consequentemente, esta mudança preserva a propriedade anterior.

Estamos agora livres para construir uma solução singular do operador L, assumindo 1 e 2 .

Considere $f$ dada por sua série parcial de Fourier

$$
f(t, x)=\sum_{n=1}^{\infty} f_{n}(t) e^{i n x}
$$

onde

$$
\begin{gathered}
f_{n}(t)=\left(1-e^{-i n c_{0} 2 \pi}\right) e^{-n \psi(t)}, n \in \mathbb{N}^{*} \\
\psi(t)=B\left(t^{*}\right)+K(1-\cos t)+i\left(a(0) \operatorname{sen} t-A\left(t^{*}\right)\right) .
\end{gathered}
$$

Como $f_{n}(t) \in C^{\omega}(\mathbb{T}), n \in \mathbb{N}^{*}$, para verificar que $f \in C^{\omega}\left(\mathbb{T}^{2}\right)$, é preciso exibir constantes $\mu, \delta, \alpha>0$ de modo que 


$$
\left|\mathrm{f}_{\mathrm{n}}(\mathrm{t}+\mathrm{is})\right| \leq \alpha \mathrm{e}^{-\mu \mathrm{n}}, \quad \forall \mathrm{t}+\mathrm{is} \in[0,2 \pi] \times(-\delta, \delta), \quad \forall \mathrm{n} \in \mathbb{N}^{*}
$$

Temos $\left|f_{n}(t+i s)\right|=\left|1-e^{-i n c_{0} 2 \pi}\right| e^{-n \operatorname{Re}(\psi(t+i s))} \leq 2 e^{-n \operatorname{Re}(\psi(t+i s))}$. Portanto, basta exibir $\mu, \delta>0$ tais que $\operatorname{Re}(\psi(t+i s)) \geq \mu$, para todo $t+i s \in[0,2 \pi] \times(-\delta, \delta)$.

Note primeiro que

$$
\begin{gathered}
2 \cos (t+i s)=e^{i(t+i s)}+e^{-i(t+i s)}=e^{-s} e^{i t}+e^{s} e^{-i t}= \\
=e^{-s}(\cos t+i \operatorname{sen} t)+e^{s}(\cos t-i \operatorname{sen} t)= \\
=\cos t\left(e^{s}+e^{-s}\right)+i \operatorname{sen} t\left(e^{-s}-e^{s}\right) . \\
2 i \operatorname{sen}(t+i s)=e^{i(t+i s)}-e^{-i(t+i s)}=e^{-s} e^{i t}-e^{s} e^{-i t}= \\
=e^{-s}(\cos t+i \operatorname{sen} t)-e^{s}(\cos t-i \operatorname{sen} t)= \\
=\cos t\left(e^{s}-e^{-s}\right)+i \operatorname{sen} t\left(e^{-s}+e^{s}\right) .
\end{gathered}
$$

Com isso, temos

$$
\begin{gathered}
\operatorname{Re}(\psi(t+i s))=\operatorname{Re}\left(B\left(t^{*}\right)+K(1-\cos (t+i s))+i\left(a(0) \operatorname{sen}(t+i s)-A\left(t^{*}\right)\right)\right)= \\
=B\left(t^{*}\right)+K-K \operatorname{Re}(\cos (t+i s))+a(0) \operatorname{Re}(i \operatorname{sen}(t+i s))= \\
=B\left(t^{*}\right)+K-K \cos t \cosh s+a(0) \cos t \operatorname{senh} s= \\
=B\left(t^{*}\right)+K(1-\cos t \cosh s)+a(0) \cos t \operatorname{senh} s .
\end{gathered}
$$

Como senh e $1-$ cosh são funções reais contínuas que se anulam no zero, existe $\delta(\mathrm{K})>0$ tal que $|\mathrm{s}|<\delta$ implica

$$
\begin{gathered}
|a(0)||\operatorname{senh} s|<\frac{B\left(t^{*}\right)}{4} \\
|1-\cosh s|<\frac{B\left(t^{*}\right)}{4 K}
\end{gathered}
$$

Assim temos que

$$
\begin{gathered}
a(0) \cos t \operatorname{senh} s>-\frac{B\left(t^{*}\right)}{4}, \\
K(1-\cos t \cosh s)>-\frac{B\left(t^{*}\right)}{4}
\end{gathered}
$$

e, portanto, vale

$$
\begin{gathered}
\operatorname{Re}(\psi(t+i s))=B\left(t^{*}\right)+K(1-\cos t \cosh s)+a(0) \cos t \operatorname{senh} s> \\
>B\left(t^{*}\right)-\frac{B\left(t^{*}\right)}{4}-\frac{B\left(t^{*}\right)}{4}=\frac{B\left(t^{*}\right)}{2}=\mu>0 .
\end{gathered}
$$


Se $u \in \mathcal{D}^{\prime}\left(\mathbb{T}^{2}\right), u=\sum_{n=1}^{\infty} u_{n}(t) e^{i n x}$ e Lu $=f$, então

$$
\begin{gathered}
u_{n}(t)= \\
=\int_{0}^{2 \pi} e^{-n\left\{B\left(t^{*}\right)-B(t)+B(t-s)+K(1-\cos (t-s))+i\left(a(0) \operatorname{sen}(t-s)-A\left(t^{*}\right)+A(t)-A(t-s)\right)\right\}} d s .
\end{gathered}
$$

Vamos mostrar que $u \in \mathcal{D}^{\prime}\left(\mathbb{T}^{2}\right)$, mas $u \notin \mathrm{C}^{\omega}\left(\mathbb{T}^{2}\right)$.

Faça $\zeta(t, s)=B\left(t^{*}\right)-B(t)+B(t-s)+K(1-\cos (t-s))$. Vamos mostrar que $\zeta(t, s) \geq 0, \forall(t, s) \in[0,2 \pi]^{2}$, e com isso

$$
\begin{gathered}
\left|\mathrm{u}_{\mathrm{n}}(\mathrm{t})\right|= \\
=\left|\int_{0}^{2 \pi} \mathrm{e}^{-\mathrm{n}\left\{\mathrm{B}\left(\mathrm{t}^{*}\right)-\mathrm{B}(\mathrm{t})+\mathrm{B}(\mathrm{t}-\mathrm{s})+\mathrm{K}\left(1-\cos (\mathrm{t}-\mathrm{s})+\mathrm{i}\left(\mathrm{a}(0) \operatorname{sen}(\mathrm{t}-\mathrm{s})-\mathrm{A}\left(\mathrm{t}^{*}\right)+\mathrm{A}(\mathrm{t})-\mathrm{A}(\mathrm{t}-\mathrm{s})\right)\right\}\right.} \mathrm{ds}\right| \leq \\
\leq \int_{0}^{2 \pi} \mathrm{e}^{-\mathrm{n \zeta}(\mathrm{t}, \mathrm{s})} \mathrm{d} s \leq \int_{0}^{2 \pi} \mathrm{d} s=2 \pi
\end{gathered}
$$

que implica $u \in \mathcal{D}^{\prime}\left(\mathbb{T}^{2}\right)$.

Note primeiro que $1-\cos (t-s)=0$ se e somente se $t=s$ ou $(t, s)=(0,2 \pi)$ ou $(t, s)=(2 \pi, 0)$. Temos também $\zeta(0,2 \pi)>0, \zeta(2 \pi, 0)>0$, e $\zeta\left(t^{*}, t^{*}\right)=0$.

Pela proposição 6.23, existem $\mathrm{K}, \varepsilon>0$, tal que $|x|<\varepsilon$ implica que $\mathrm{B}(\mathrm{x})+\mathrm{K}(1-$ $\cos x) \geq 0$.

Seja $E=\{(t, t+r)|t \in[0,2 \pi]| r \mid,<\varepsilon\} \cap[0,2 \pi]^{2}$, com isso $(t, s) \in E$ implica que $|t-s|<\varepsilon$. Defina $F=(B((0,2 \pi), \xi) \cup B((2 \pi, 0), \xi)) \cap[0,2 \pi]^{2}$, onde as bolas $\mathrm{B}((0,2 \pi), \xi)$ e $\mathrm{B}((2 \pi, 0), \xi)$ conservam o sinal (positivo) de $\zeta$.

Desta forma, se $G=[0,2 \pi]^{2}-E \cup F$, então $\min _{(t, s) \in G}\{1-\cos (t-s)\}>0$, e existe $K>0$ tal que $\forall(t, s) \in G$,

$$
\begin{gathered}
\mathrm{K}(1-\cos (\mathrm{t}-\mathrm{s})) \geq \mathrm{K} \min _{(\mathrm{t}, \mathrm{s}) \in \mathrm{G}}\{1-\cos (\mathrm{t}-\mathrm{s})\} \geq \max _{(\mathrm{t}, \mathrm{s}) \in \mathrm{G}}\{-\mathrm{B}(\mathrm{t}-\mathrm{s})\} \geq \\
\geq-\mathrm{B}(\mathrm{t}-\mathrm{s}) \Longrightarrow \mathrm{K}(1-\cos (\mathrm{t}-\mathrm{s}))+\mathrm{B}(\mathrm{t}-\mathrm{s})) \geq 0 .
\end{gathered}
$$

Temos $B\left(t^{*}\right)-B(t) \geq 0, \forall t \in[0,2 \pi]$ e, portanto,

$$
\begin{gathered}
(\mathrm{t}, \mathrm{s}) \in \mathrm{E} \cup \mathrm{F} \cup \mathrm{G}=[0,2 \pi]^{2} \Longrightarrow \mathrm{B}(\mathrm{t}-\mathrm{s})+\mathrm{K}(1-\cos (\mathrm{t}-\mathrm{s})) \geq 0 \Longrightarrow \\
\Longrightarrow \zeta(\mathrm{t}, \mathrm{s})=\mathrm{B}\left(\mathrm{t}^{*}\right)-\mathrm{B}(\mathrm{t})+\mathrm{B}(\mathrm{t}-\mathrm{s})+\mathrm{K}(1-\cos (\mathrm{t}-\mathrm{s})) \geq 0 .
\end{gathered}
$$

Falta mostrar que $u \notin C^{\omega}\left(\mathbb{T}^{2}\right)$. Vamos verificar que $\left|u_{n}\left(t^{*}\right)\right|$ não decai exponencialmente, ou seja, não vale $\left|u_{n}\left(t^{*}\right)\right| \leq e^{-n \alpha}, \forall n \in \mathbb{N}^{*}$, para qualquer escolha de $\alpha>0$.

Defina

$$
\begin{gathered}
\varphi^{*}(t, s)=B\left(t^{*}\right)-B(t)+B(t-s)+K(1-\cos (t-s))+ \\
+i\left(a(0) \operatorname{sen}(t-s)-A\left(t^{*}\right)+A(t)-A(t-s)\right) .
\end{gathered}
$$


Assim temos

$$
\begin{gathered}
\varphi^{*}\left(t^{*}, s\right)=B\left(t^{*}\right)-B\left(t^{*}\right)+B\left(t^{*}-s\right)+K\left(1-\cos \left(t^{*}-s\right)\right)+ \\
+i\left(a(0) \operatorname{sen}\left(t^{*}-s\right)-A\left(t^{*}\right)+A\left(t^{*}\right)-A\left(t^{*}-s\right)\right)= \\
=B\left(t^{*}-s\right)+K\left(1-\cos \left(t^{*}-s\right)\right)+i\left(a(0) \operatorname{sen}\left(t^{*}-s\right)-A\left(t^{*}-s\right)\right) . \\
u_{n}\left(t^{*}\right)=\int_{0}^{2 \pi} e^{-n \varphi^{*}\left(t^{*}, s\right)} d s=\int_{\substack{\mid t t^{*}-s \leq \leq \varepsilon \\
s \in[0,2 \pi]}} e^{-n \varphi^{*}\left(t^{*}, s\right)} d s+\int_{\substack{\left|t^{*}-s\right| \geq \varepsilon \\
s \in[0,2 \pi]}} e^{-n \varphi^{*}\left(t^{*}, s\right)} d s .
\end{gathered}
$$

para algum $\varepsilon>0$ a ser escolhido posteriormente. Podemos assumir que $K>0$ satisfaz

$$
\begin{gathered}
\mathrm{K}\left(1-\cos \left(\mathrm{t}^{*}-\mathrm{s}\right)\right)-\mathrm{B}\left(\mathrm{t}^{*}-\mathrm{s}\right) \geq \mathrm{K} \min _{\substack{\left|\mathrm{t}^{*}-s\right| \geq \varepsilon \\
s \in[0,2 \pi]}}\left\{1-\cos \left(\mathrm{t}^{*}-\mathrm{s}\right)\right\}- \\
-\max _{\substack{\left|\mathrm{t}^{*}-s\right| \geq \varepsilon \\
\mathrm{s} \in[0,2 \pi]}}\left\{\mathrm{B}\left(\mathrm{t}^{*}-\mathrm{s}\right)\right\} \geq 0 .
\end{gathered}
$$

Portanto,

$$
\int_{\substack{\left|t^{*}-s\right| \geq \varepsilon \\ s \in[0,2 \pi]}} e^{-n \varphi^{*}\left(t^{*}, s\right)} d s=O\left(e^{-\beta n}\right)
$$

para algum $\beta>0$.

Faça agora $\varphi(\sigma)=\varphi^{*}\left(t^{*}, t^{*}-\sigma\right)$. Com isso,

$$
\int_{\substack{\left|t^{*}-s\right| \leq \varepsilon \\ s \in[0,2 \pi]}} e^{-n \varphi^{*}\left(t^{*}, s\right)} d s=\int_{|\sigma| \leq \varepsilon} e^{-n \varphi(\sigma)} d \sigma .
$$

Vamos utilizar o método da fase estacionária 5.6.

$$
\begin{gathered}
\varphi(\sigma)=\mathrm{K}(1-\cos \sigma)+i(\mathrm{C}(\sigma)+\mathrm{a}(0) \operatorname{sen} \sigma) \\
\varphi^{\prime}(\sigma)=\mathrm{K} \operatorname{sen} \sigma+i(\mathrm{c}(\sigma)-\mathrm{a}(0) \cos \sigma) \\
\varphi^{\prime \prime}(\sigma)=-\mathrm{K} \cos \sigma+i\left(\mathrm{c}^{\prime}(\sigma)+\mathrm{a}(0) \operatorname{sen} \sigma\right)
\end{gathered}
$$

e, portanto,

$$
\varphi(0)=0, \quad \varphi^{\prime}(0)=-b(0)=0, \quad \varphi^{\prime \prime}(0)=-\mathrm{K}+i c^{\prime}(0) \neq 0, \text { pois } \mathrm{K} \neq 0 .
$$

Pela proposição 6.22, podemos escolher $\varepsilon>0$ de tal forma que

$$
\operatorname{Re}(\varphi(\sigma))=\mathrm{K}(1-\cos (\sigma))-\mathrm{B}(\sigma) \geq 0,
$$




$$
\begin{gathered}
\operatorname{Re}(\varphi(\varepsilon)) \neq 0, \\
\operatorname{Re}(\varphi(-\varepsilon)) \neq 0 .
\end{gathered}
$$

Desta forma, o método da fase estacionária nos dá, com $\gamma \in \mathbb{C}$

$$
\begin{gathered}
\left|\mathrm{u}_{\mathrm{n}}\left(\mathrm{t}^{*}\right)\right|=\int_{|\sigma| \leq \varepsilon} e^{-\mathrm{n} \varphi(\sigma)} \mathrm{d} \sigma+\int_{\substack{\left|\mathrm{t}^{*}-s\right| \geq \varepsilon \\
s \in[0,2 \pi]}} e^{-\mathrm{n} \varphi^{*}\left(\mathrm{t}^{*}, \mathrm{~s}\right)} \mathrm{ds}= \\
=\gamma \mathrm{n}^{-\frac{1}{2}}\left(1+\mathrm{O}\left(\mathrm{n}^{-1}\right)+\mathrm{O}\left(\mathrm{e}^{-\mathrm{n} \beta}\right)\right)
\end{gathered}
$$

Suponha agora que existe $\rho, M>0$, tal que para todo $n \in \mathbb{N}^{*}$

$$
\left|\gamma n^{-\frac{1}{2}}\left(1+\mathrm{O}\left(\mathrm{n}^{-1}\right)+\mathrm{O}\left(\mathrm{e}^{-\mathrm{n} \beta}\right)\right)\right| \leq M e^{-n \rho}
$$

Então

$$
\left|e^{\mathfrak{n} \rho} \gamma \mathrm{n}^{-\frac{1}{2}}\left(1+\mathrm{O}\left(\mathrm{n}^{-1}\right)+\mathrm{O}\left(\mathrm{e}^{-\mathrm{n} \beta}\right)\right)\right| \leq \mathrm{M}
$$

O que é falso, pois o limite do lado esquerdo da desigualdade tende a $+\infty$, quando $n$ tende e a $+\infty$. Com isso concluímos que $u \notin \mathrm{C}^{\omega}\left(\mathbb{T}^{2}\right)$.

\section{Construção de uma Solução Singular pelo Método da Maior Descida}

A argumentação usada aqui é a mesma apresentada em [B2]. A construção de $f$ é de tal forma que o comportamento assintótico dos coeficientes parciais de $u$ é obtido através de uma integral dependente de um parâmetro (o índice do coeficiente), que possibilita a aplicação do método da maior descida. Desse maneira é possível obtermos uma expressão para a ordem (não exponencial) do decaimento dos coeficientes parciais de $u$.

Considere $f \in \mathcal{D}^{\prime}\left(\mathbb{T}^{2}\right)$ dada pela série parcial de Fourier

$$
\begin{gathered}
f(t, x)=(2 \pi)^{-1} \sum_{n=1}^{\infty} f_{n}(t) e^{i n x} \\
f_{n}(t)=\left(1-e^{-i 2 \pi n c_{0}}\right) e^{-n(A+K G(t)-\varepsilon)} \\
G(t)=1-\cos \left(t-t_{0}+s_{0}\right) .
\end{gathered}
$$

Os números reais $A, K, \varepsilon, t_{0}, s_{0}$ serão escolhidos no que segue de modo a obtermos $f \in C^{\omega}\left(\mathbb{T}^{2}\right)$.

Defina $A=\max \left\{\int_{t-s}^{t} b ; 0 \leq s, t \leq 2 \pi\right\}$. Seja $\left(t_{0}, s_{0}\right)$ onde o máximo é atingido, e desta forma, $A=\int_{t_{0}-s_{0}}^{t_{0}} b$. Podemos assumir que $b(0)>0$, pois nenhuma translação 
de $b$ altera o valor de $b_{0}$, e nenhuma translação de $b$ altera a hipoeliticidade analítica global de L, como foi visto em 6.20, página 90. Também podemos assumir que $0<t_{0}, s_{0}, t_{0}-s_{0}<2 \pi$.

Temos que $0<c_{1}=1-e^{2 \pi b_{0}} \leq\left|1+e^{-i 2 \pi n c_{0}}\right| \leq 1+e^{2 \pi b_{0}}=c_{2}$, para todo $n \in \mathbb{N}$. Além disto, vale a estimativa $\left|f_{n}(t)\right| \leq c_{2} e^{-n(A-\varepsilon-K(\cosh \delta-1))}$, para todo $n \in \mathbb{N}$, $t \in[0,2 \pi] \times(-\delta, \delta)$, já que a estimativa anterior é equivalente a $\cosh \delta \geq \operatorname{Re}(\cosh (\mathrm{t}-$ $\left.\left.t_{0}+s_{0}\right)\right)$.

$\mathrm{O}$ valor necessário de $\mathrm{K}>0$, vai aumentando conforme prosseguimos na demonstração, mas desde que tenhamos $\varepsilon=\mathrm{O}\left(\mathrm{K}^{-1}\right), \delta=\mathrm{O}\left(\mathrm{K}^{-1}\right)$, concluímos $A-\varepsilon-$ $\mathrm{K}(\cosh \delta-1) \geq A-\varepsilon-2 K \delta^{2}=A-O\left(K^{-1}\right) \geq \frac{A}{2}$, se $K$ é grande o suficiente.

Do discutido acima segue que $\left|f_{n}(t)\right| \leq c_{2} e^{\frac{-A n}{2}}$, para todo $n \in \mathbb{N}, t \in[0,2 \pi] \times$ $(-\delta, \delta)$. Como cada $f_{n} \in C^{\omega}(\mathbb{T})$, verificamos que $f \in C^{\omega}\left(\mathbb{T}^{2}\right)$.

Nosso objetivo no que segue é mostrar que se $f$ é como acima, então a solução $u$ de $L u=f$ não é uma função analítica real, ou seja, seus coeficientes parciais de Fourier não decaem uniformemente exponencialmente.

Considere o conjunto $Y=\left\{0 \leq t \leq 2 \pi: \int_{t_{0}-s_{0}}^{t} b=A\right\}$. Como $b$ é uma função analítica real não nula, $\int_{t_{0}-s_{0}}^{t} b-A$ tem um número finito de zeros em $[0,2 \pi]$, ou seja, $\mathrm{Y}=\left\{\mathrm{t}_{0}<\cdots<\mathrm{t}_{\mathrm{r}}\right\}$.

Note agora que $\int_{t_{0}-s_{0}}^{t_{k}} b+\int_{t_{k}}^{t_{0}} b=\int_{t_{0}-s_{0}}^{t_{0}} b$ implica que $\int_{t_{0}}^{t_{k}} b=0, k=1, \ldots, r$. Temos também que $b$ muda de sinal de + para - em $t_{k}, k=1, \ldots, r$, pois se $b$ é positivo numa vizinhança à esquerda de $t_{k}$, então a integral de $b$ aumenta, se o extremo superior for $t_{0}$, e extremo inferior pertencer a essa vizinhança, o que contraria o fato do máximo ser atingido em $\left(t_{0}, s_{0}\right)$. De maneira análoga concluímos que $b$ é positiva à direita de $t_{0}-s_{0}$, o que implica que $b$ muda de sinal de - para + em $t_{0}-s_{0}$.

Tome $\rho$ de tal forma que $t_{k}$ seja o único zero de $\int_{t_{0}-s_{0}}^{t} b-A$ em $\left\{t ;\left|t-t_{k}\right| \leq \rho\right\}$, $k=1, \ldots, r$. Desta forma

$$
\int_{t_{0}}^{t} b+\int_{t}^{t_{0}-s_{0}} b=-A \Longrightarrow \int_{t_{0}}^{t} b=-A+\int_{t_{0}-s_{0}}^{t} b \leq 0, t \in\left\{t ;\left|t-t_{k}\right| \leq \rho\right\} .
$$

De acordo com o lema 6.8 , se $u \in \mathcal{D}^{\prime}\left(\mathbb{T}^{2}\right)$ é tal que $L u=f$, então os coeficientes parciais de $u$ são dados por

$$
u_{n}(t)=\int_{0}^{2 \pi} e^{-n\left(A+K G(t-s)-\varepsilon+i \int_{t-s}^{t} c\right)} d s .
$$

para $n>0$. Note que cada $u_{n} \in C^{\omega}(\mathbb{T})$.

No que segue, vamos mostrar que $u \in \mathcal{D}^{\prime}\left(\mathbb{T}^{2}\right) \backslash C^{\omega}\left(\mathbb{T}^{2}\right)$. Para $k=0, \ldots, r$ e $x \in \mathbb{T}$, $\left(t_{k}, x\right)$ pertence ao suporte singular analítico de $u$ (o complementar da união de todos os abertos onde $u$ é analítica). Basta verificarmos, que $u$ é uma distribuição e que $\left|u_{n}\left(t_{k}\right)\right|$ não decai exponencialmente. 
Chegamos aqui na parte mais elaborada da demonstração. O comportamento assintótico da integral envolvida no cálculo de $u_{n}(t)$, nos permitirá concluir que existem $C>0, \gamma_{1}>0$, tais que

$$
\left|u_{n}(t)\right| \leq C n^{-\frac{1}{2}}
$$

para todo $t \in \mathbb{T}, n>0$. Além disto, vamos mostrar que

$$
\left|u_{n}\left(t_{k}\right)\right|=\gamma_{1} n^{-\frac{1}{2}}\left(1+O\left(n^{-1}\right)\right)
$$

para $k=0, \ldots, r, n \longrightarrow \infty$.

Considere a mudança de variáveis $\sigma=t-s$ na fórmula 6.33. Assim obtemos

$$
u_{n}(t)=\int_{t-2 \pi}^{t} e^{-n\left(A+K G(\sigma)-\varepsilon+i \int_{\sigma}^{t} c\right) d \sigma} .
$$

Fazendo

$$
\tilde{u}_{n}(t)=\int_{t-2 \pi}^{t} e^{-n\left(K\left(1-\cos \left(\sigma-\sigma_{0}\right)\right)-i \int_{\sigma_{0}}^{\sigma} c\right)} d \sigma
$$

onde $\sigma_{0}=t_{0}-s_{0}$, obtemos

$$
u_{n}(t)=\tilde{u}_{n}(t) e^{-n\left(A-\varepsilon+i \int_{\sigma_{0}}^{t} c\right)} .
$$

Defina $h(\sigma)=K\left(1-\cos \left(\sigma-\sigma_{0}\right)\right)-i \int_{\sigma_{0}}^{\sigma} c$, para $t-2 \pi \leq \sigma \leq t$, com isso, estamos, estamos nos preparando para usar o método da maior descida.Como $\cos ^{\prime}(0)=0$, usando a proposição 6.23, podemos concluir que se $\mathrm{K}_{0}$ é grande o suficiente, então para todo $K \geq K_{0}$, Re $h$ é estritamente decrescente em $\left[\sigma_{0}-\frac{\pi}{2}, \sigma_{0}\right] \cap[t-2 \pi, t]$, e estritamente crescente em $\left[\sigma_{0}, \sigma_{0}+\frac{\pi}{2}\right] \cap[t-2 \pi, t]$, para todo $t \in[0,2 \pi]$. Também é verdade que existe $C_{1}>0$ tal que $\operatorname{Re} h \geq C_{1}$ em $\left\{\sigma ;\left|\sigma-\sigma_{0}\right| \geq \frac{\pi}{2} \cap[t-2 \pi, t]\right\}$, para todo $t \in[0,2 \pi]$. De fato, como $0<\sigma_{0}<2 \pi$, temos $\sigma_{0}+2 \pi \notin[t-2 \pi$, $t]$, e portanto $K\left(1-\cos \left(\sigma-\sigma_{0}\right)\right)>0$ em $\left\{\sigma ;\left|\sigma-\sigma_{0}\right| \geq \frac{\pi}{2} \cap[t-2 \pi, t]\right\}$, e com isso $K_{0}$ pode ser escolhido de tal forma que

$$
\mathrm{K} \min _{\sigma \in[0,2 \pi] \cap\left\{\sigma ;\left|\sigma-\sigma_{0}\right| \geq \frac{\pi}{2}\right\}}\left\{1-\cos \left(\sigma-\sigma_{0}\right)\right\}>\max _{\sigma \in[-2 \pi, 2 \pi]}\left\{-\int_{\sigma_{0}}^{\sigma} b\right\},
$$

para $\mathrm{K} \geq \mathrm{K}_{0}$.

Seja $D=\cup_{k=1}^{r}\left\{t ;\left|t-t_{k}\right| \leq \rho\right\}$. Podemos assumir que $\rho$ é pequeno o suficiente para termos $t \in D \Longrightarrow t \neq \sigma_{0}$, e com isso, se $t \in D$, então $t-2 \pi<\sigma_{0}<t$, e $\int_{t_{0}}^{t} b \leq 0$.

Para obtermos o comportamento assintótico de $\left|u_{n}(t)\right|, n \longrightarrow \infty$, devemos considerar separadamente, quando $t \in D$, e quando $t \in[0,2 \pi] \backslash D$. Para $t \in D$, obteremos 6.34, e uma parte de 6.33 Se $t \in[0,2 \pi] \backslash D$, concluímos a outra parte de 6.33.

Seja $t \in D$. 
Tome $\sigma_{1}, \sigma_{2}$, tais que $\left[\sigma_{1}, \sigma_{2}\right] \cap \mathrm{D}=\emptyset, \sigma_{1}<\sigma_{0}<\sigma_{2}$ e $\left[\sigma_{1}, \sigma_{2}\right] \subset\left[\sigma_{0}-\frac{\pi}{2}, \sigma_{0}+\frac{\pi}{2}\right] \cap$ $[t-2 \pi, t]$. Para ver que isso é possível, note que $\sigma_{0}<t_{0}<\cdots<t_{r}$ e, portanto, é suficiente que $\sigma_{2}-\sigma_{0}<\mathrm{d}\left(\sigma_{0}, \mathrm{D}\right)$ e $\sigma_{0}-\sigma_{1}<\mathrm{d}\left(\sigma_{0},-2 \pi+\mathrm{D}\right)$.

Defina

$$
\mathrm{J}_{\mathrm{n}}=\int_{\sigma_{1}}^{\sigma_{2}} e^{-\mathrm{nh}(\sigma)} \mathrm{d} \sigma
$$

Estamos nas condições do método da maior descida, e portanto

$$
\mathrm{J}_{\mathrm{n}}=\gamma \mathrm{n}^{-\frac{1}{2}}\left(1+\mathrm{O}\left(\mathrm{n}^{-1}\right)\right) e^{-\mathrm{nh}(0)} .
$$

No que segue, vamos estimar $\left|u_{n}(t)\right|$. Note que

$$
\begin{gathered}
\left|\int_{t-2 \pi}^{\sigma_{1}} e^{-n h(\sigma)} d \sigma\right| \leq \int_{t-2 \pi}^{\sigma_{1}} e^{-n R e h(\sigma)} d \sigma \leq e^{-C_{1} n} \\
\left|\int_{\sigma_{2}}^{t} e^{-n h(\sigma)} d \sigma\right| \leq \int_{\sigma_{2}}^{t} e^{-n \operatorname{Re}(h \sigma)} d \sigma \leq e^{-C_{1} n} .
\end{gathered}
$$

Já podemos concluir, que

$$
\begin{gathered}
u_{n}(t)=\int_{t-2 \pi}^{t} e^{-n\left(A+K G(\sigma)-\varepsilon+i \int_{\sigma}^{t} c\right) d \sigma}= \\
=e^{-n\left(A-\varepsilon+i \int_{\sigma_{0}}^{t} c\right)} \int_{t-2 \pi}^{t} e^{-n\left(K\left(1-\cos \left(\sigma-\sigma_{0}\right)\right)-i \int_{\sigma_{0}}^{\sigma} c\right)} d \sigma= \\
=e^{-n\left(A-\varepsilon+i \int_{\sigma_{0}}^{t} c\right)} \int_{t-2 \pi}^{t} e^{-n h(\sigma)} d \sigma= \\
=e^{-n\left(A-\varepsilon+i \int_{\sigma_{0}}^{t} c\right)}\left(J_{n}+\int_{t-2 \pi}^{\sigma_{1}} e^{-n h(\sigma)} d \sigma+\int_{\sigma_{2}}^{t} e^{-n h(\sigma)} d \sigma\right) .
\end{gathered}
$$

É necessário agora definirmos $\varepsilon=\operatorname{Re} h(0)>0$. Dessa maneira, obtemos

$$
\begin{gathered}
\left|u_{n}(t)\right|=\left|e^{-n\left(A-\varepsilon+i \int_{\sigma_{0}}^{t} c\right)}\right|\left(e^{-n \operatorname{Re} h(0)}|\gamma| n^{-\frac{1}{2}}\left(1+O\left(n^{-1}\right)\right)+O\left(e^{-C_{1} n}\right)\right)= \\
=e^{-n\left(A-\int_{\sigma_{0}}^{t} b\right)}\left(|\gamma| n^{-\frac{1}{2}}\left(1+O\left(n^{-1}\right)\right)+O\left(e^{-C_{1} n}\right)\right)= \\
=e^{n \int_{t_{0}}^{t} b}\left(|\gamma| n^{-\frac{1}{2}}\left(1+O\left(n^{-1}\right)\right)+O\left(e^{-C_{1} n}\right)\right) .
\end{gathered}
$$

Faça $\gamma_{1}=|\gamma|$. Se $t \in D$, então $\int_{t_{0}}^{t} b \leq 0$. Temos que $\left|O\left(e^{-C_{1} n}\right)\right| \leq C_{2} n^{-\frac{1}{2}}$, para algum $\mathrm{C}_{2}>0$. Portanto

$$
\begin{gathered}
\left|\mathrm{u}_{\mathrm{n}}(\mathrm{t})\right| \leq \gamma_{1} e^{\mathrm{n} \int_{\mathrm{t}_{0}}^{\mathrm{t}} \mathrm{b}}\left|\left(1+\mathrm{O}\left(\mathrm{n}^{-1}\right)\right)\right| \mathrm{n}^{-\frac{1}{2}}+\left|\mathrm{O}\left(\mathrm{e}^{-\mathrm{C}_{1} \mathrm{n}}\right)\right| \leq \\
\leq\left(\gamma_{1} e^{n \int_{\mathrm{t}_{0}}^{t} b^{b}}\left|\left(1+\mathrm{O}\left(\mathrm{n}^{-1}\right)\right)\right|+\mathrm{C}_{2}\right) \mathrm{n}^{-\frac{1}{2}} \leq \mathrm{C} n^{-\frac{1}{2}},
\end{gathered}
$$


para uma escolha apropriada de $C>0$. Isso nos dá 6.33 para $t \in \mathrm{D}$.

De $\int_{t_{0}}^{t_{k}} b=0$, para $k=0, \ldots, r$, segue que

$$
\left|u_{n}\left(t_{k}\right)\right|=\gamma_{1} n^{-\frac{1}{2}}\left(1+O\left(n^{-1}\right)\right)+O\left(e^{-C_{1} n}\right),
$$

o que garante 6.34 .

No que segue, devemos mostrar que vale 6.34 quando $t \in[0,2 \pi] \backslash D$.

Note que o máximo da função $\int_{\sigma_{0}}^{t} b-A$ é negativo em $[0,2 \pi] \backslash \cup_{k=1}^{r}\left\{t ;\left|t-t_{k}\right|<\rho\right\}$, e portanto existe $\eta>0$ tal que $\int_{\sigma_{0}}^{t} b-A \leq-\eta$ implica $A-\int_{\sigma_{0}}^{t} b \geq \eta$, para todo $t \in[0,2 \pi] \backslash D$. Além disto, não altera o demonstrado até aqui, se considerarmos $K_{0}$ maior, se necessário, de tal forma que $\mathrm{K} \geq \mathrm{K}_{0}$ implica $\varepsilon \leq-\frac{\eta}{2}$, pois $\varepsilon=\mathrm{O}\left(\mathrm{K}^{-1}\right)$. Dessa maneira, obtemos

$$
\operatorname{Re}\left(A-\varepsilon+i \int_{\sigma_{0}}^{t} c\right)=A-\varepsilon-\int_{\sigma_{0}}^{t} b \geq \frac{\eta}{2} .
$$

Devemos mostrar agora que, aumentando o valor de $K_{0}$, se necessário, temos que, se $K \geq K_{0}, \sigma \in[t-2 \pi, t], t \in[0,2 \pi] \backslash D$, então $\operatorname{Re} h(\sigma) \geq 0$.

Observe que b necessariamente muda de sinal de - para + em $\sigma_{0}$, caso contrário, $\sigma_{0}$ não seria o máximo como foi definido. Isso implica que existe $v>0$ tal que $\int_{\sigma_{0}}^{\sigma} b \geq 0$, se $\left|\sigma-\sigma_{0}\right| \leq v$.

Devemos considerar separadamente, para $\sigma \in[-2 \pi, 2 \pi]$, quando $\left|\sigma-\sigma_{0}\right| \nu,\left|\sigma-\left(\sigma_{0}-2 \pi\right)\right|<\nu$, ou quando não vale nenhuma dessas duas desigualdades anteriores.

Se $\left|\sigma-\sigma_{0}\right| \leq v$, então $\int_{\sigma_{0}}^{\sigma} b \geq 0$ implica $\operatorname{Re} h(\sigma)=K\left(1-\cos \left(\sigma-\sigma_{0}\right)\right)+\int_{\sigma_{0}}^{\sigma} b \geq 0$.

Se $\left|\sigma-\left(\sigma_{0}-2 \pi\right)\right|<v$, então $\int_{\sigma_{0}}^{\sigma}=-2 \pi \mathrm{b}_{0}+\int_{\sigma_{0}-2 \pi}^{\sigma} \mathrm{b}>0$ e $\operatorname{Re} h(\sigma) \geq 0$

Agora, se $\sigma \in[-2 \pi, 2 \pi],\left|\sigma-\sigma_{0}\right|>v$ e $\left|\sigma-\left(\sigma_{0}-2 \pi\right)\right|>v$, tome $K>0$, de acordo com as restrições acima de tal forma que

$$
\begin{aligned}
1-\cos \left(\sigma-\sigma_{0}\right) \geq \tilde{\eta}>0 & \Longrightarrow \operatorname{Reh}(\sigma)=\mathrm{K}\left(1-\cos \left(\sigma-\sigma_{0}\right)\right)+\int_{\sigma_{0}}^{\sigma} \mathrm{b} \geq \\
& \geq \mathrm{K} \tilde{\eta}+\int_{\sigma_{0}}^{\sigma} \mathrm{b} \geq 0 .
\end{aligned}
$$

Considerando o discutido acima, vem que, se $t \in[0,2 \pi] \backslash D$ então

$$
\begin{gathered}
\left|u_{n}(t)\right|=\left|e^{-n\left(A-\varepsilon+i \int_{\sigma_{0}}^{t} c\right)}\right|\left|\int_{t-2 \pi}^{t} e^{-n\left(K\left(1-\cos \left(\sigma-\sigma_{0}\right)\right)-i \int_{\sigma_{0}}^{\sigma} c\right)} d \sigma\right| \leq \\
\leq 2 \pi e^{-\frac{\eta}{2} n} \leq \mathrm{Cn}^{-\frac{1}{2}},
\end{gathered}
$$

aumentando o valor de $C>0$, se necessário. Isso termina a construção da solução singular do operador L, quando b muda de sinal.

Finalmente, consideremos o caso quando $b_{0}=0$, isto é quando $c_{0}=a_{0}$. Se $a_{0} \in \mathbb{Q}$ então podemos tomar $a_{k}=1, k \in \mathbb{Z}$, em 6.7 e obtemos uma solução singular. Quando 
$\mathrm{a}_{0} \in \mathbb{R} \backslash \mathbb{Q}$, podemos tomar $\mathrm{f}$ cujos coeficientes parciais de Fourier são como em 6.32. Note que $\left|1-e^{-2 \pi i n a_{0}}\right| \leq 2$. Procedemos a análise de que $u$ é uma solução singular como no caso $b_{0}<0$. 


\section{Referências Bibliográficas}

[B1] Bergamasco, A., Hipoeliticidade global para certas classes de operadores, Tese de Livre-Docência, ICMC-USP, São Carlos, SP, 1989.

[B2] Bergamasco, A., Remarks about global analytic hypoellipticity, Trans. Amer. Math. Soc. 351 (1999), 4113-4126.

[BNZ] Bergamasco, A.; Nunes, W.; Zani, S., Global analytic hypoellipticity and pseudoperiodic functions, Mat. Contemporanea 18 (2000), 43-57.

[BH] Bleistein, N; Handelsman, R. A., Asymptotic Expansions of Integrals, Second edition. Dover Publications, Inc., New York, 1986.

[C] Coffman, A., Notes on series of several variables, www.ipfw.edu/math/Coffman

[Co] Conway, J., Functions of one complex variable, Second edition, Graduate Texts in Mathematics, 11. Springer-Verlag, New York-Berlin, 1978.

[deB] de Bruijn, N., Asymptotic Methods in Analysis, Dover, New York, 1981.

[G] Greenfield, S., Hypoelliptic vector fields and continued fractions, Proc. Amer. Math. Soc. 31 (1972), 115-118.

[GW] Greenfield, S.; Wallach, N., Global hypoellipticity and Liouville numbers, Proc. Amer. Math. Soc. 31 (1972), 112-114.

[KP] Krantz, S.; Parks, H, A primer of real analytic functions, Basler Lehrbücher [Basel Textbooks], 4. Birkhäuser Verlag, Basel, 1992.

[Sj] Sjöstrand, J., Singularités analytiques microlocales, Astérisque, 95, 1-166, Soc. Math. France, Paris, 1982.

[Z] Zani, S., Hipoeliticidade Global para Operadores de Segunda Ordem, Dissertação de Mestrado, ICMC-USP, 1988. 